\title{
Power Scaling of Enhancement Cavities for Nonlinear Optics
}

loachim Pupeza

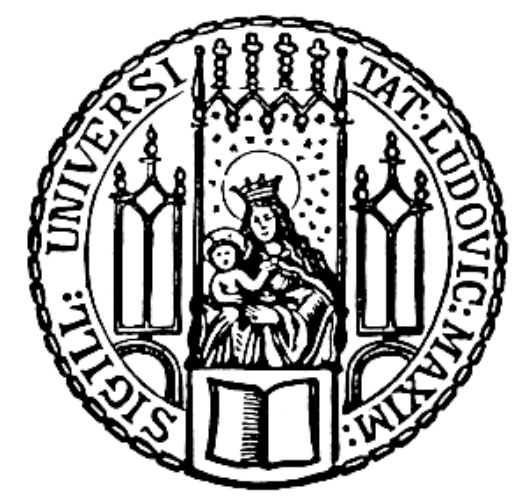

München 2011 



\title{
Power Scaling of Enhancement Cavities for Nonlinear Optics
}

loachim Pupeza

\author{
Dissertation \\ an der Fakultät für Physik \\ der Ludwig-Maximilians-Universität \\ München
}

vorgelegt von

Ioachim Pupeza

aus Bucureşti, Rumänien

München, den 8. September 2011 
Erstgutachter: Prof. Ferenc Krausz

Zweitgutachter: Prof. Theodor W. Hänsch

Tag der mündlichen Prüfung: 7. Oktober 2011 


\section{Contents}

Zusammenfassung vii

Abstract viii

\begin{tabular}{lll}
\hline & Introduction & $\mathbf{1}$
\end{tabular}

1.1 About Light: From the Genesis to the Laser . . . . . . . . . . . . . . . . . 1

1.2 Motivation: Enhancement of Nonlinear Processes

in Passive Optical Resonators . . . . . . . . . . . . . . . . . . . . 4

1.3 Overview of the Results . . . . . . . . . . . . . . . . . . . . . . . . . . . . . . . . . . . . . . .

1.4 Structure of the Thesis . . . . . . . . . . . . . . . . . . . . . 8

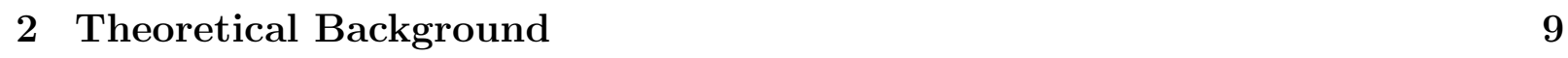

2.1 Passive Enhancement of Ultrashort Pulses . . . . . . . . . . . . . . . . . . 9

2.1 .1 Ultrashort Pulses from Mode-Locked Lasers . . . . . . . . . . . . . 9

$2.1 .2 \quad$ Passive Enhancement in an External Cavity . . . . . . . . . . . . . 13

$2.1 .3 \quad$ Interferometric Stabilization . . . . . . . . . . . . . . . . . . . 18

2.1 .4 Transverse Modes, Cavity Scan and Quasi-Imaging . . . . . . . . . 24

2.2 High-Harmonic Generation (HHG) in a Gas . . . . . . . . . . . . . . . . . 28

2.2 .1 The Three-Step Model and Phase-Matching . . . . . . . . . . . . . 28

2.2 .2 Scaling Laws . . . . . . . . . . . . . . . . . . . . . . . . . . 32

3 Objectives of the Experiment and Technological Challenges 35

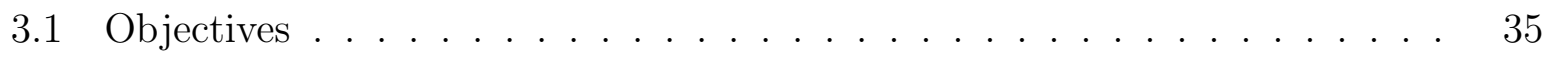

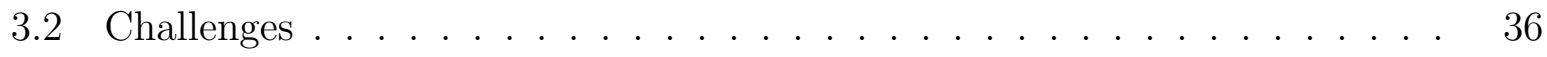

3.2 .1 Power and Bandwidth Scaling of an Empty Cavity . . . . . . . . . 36

3.2 .2 Inclusion of a Gas Target . . . . . . . . . . . . . . . . . . . . . . . . . . . . . . . . . 37

3.2 .3 XUV Output Coupling . . . . . . . . . . . . . . . . . . . . . . 39

\begin{tabular}{|lll}
\hline & Experimental Setup and Results & 41
\end{tabular}

4.1 Yb-Based CPA System with $80 \mathrm{MHz}, 200 \mathrm{fs}, 50 \mathrm{~W}$. . . . . . . . . . . . . . 41

4.1 .1 The Oscillator . . . . . . . . . . . . . . . . . . . . . 41

4.1 .2 Chirped-Pulse Amplification . . . . . . . . . . . . . . . . . . 44

4.2 The Enhancement Cavity . . . . . . . . . . . . . . . . . . . . . 45 


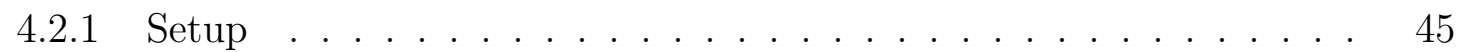

4.2 .2 Diagnostics . . . . . . . . . . . . . . . . . . . . . . . 46

4.3 Power Scaling of the Empty Cavity . . . . . . . . . . . . . . . . . . . . . . . . . 51

4.4 XUV Output Coupling Techniques . . . . . . . . . . . . . . . . . . . . 53

4.4 .1 Brewster Plates . . . . . . . . . . . . . . . . . . . 54

$4.4 .2 \quad$ WOMOC: Wedge-On-Mirror Output Coupler . . . . . . . . . . . 58

$4.4 .3 \quad$ Direct On-Axis Access . . . . . . . . . . . . . . . . 58

4.4 .4 Other Methods . . . . . . . . . . . . . . . . . . . . . 59

4.5 XUV Generation . . . . . . . . . . . . . . . . . . . . . . . . . . . . . . . . 60

4.5 .1 Intracavity Gas Target . . . . . . . . . . . . . . . . . . . . 60

4.5 .2 XUV Diagnostics . . . . . . . . . . . . . . . . . . . . . 61

4.5 .3 XUV Generation - First Results . . . . . . . . . . . . . . . . . . . . 62

\begin{tabular}{|lll}
5 & Outlook & 67 \\
\hline
\end{tabular}

5.1 Further Power Scaling of Enhancement Cavities . . . . . . . . . . . . . . . 67

5.1 .1 Novel Cavity Designs . . . . . . . . . . . . . . . . . . . . . . . . . . . . . . . 67

$5.1 .2 \quad$ Novel Laser Sources . . . . . . . . . . . . . . . . . . . . . . . . . . . 70

5.2 Power Scalable XUV Output Coupling . . . . . . . . . . . . . . . . . . . . . . 72

5.2 .1 Thin Plates and WOMOC . . . . . . . . . . . . . . . . . . . . . 73

5.2 .2 Mirrors with Apertures, Direct Output Coupling. . . . . . . . . . . 73

5.3 Towards Isolated as-Pulses Using fs-Enhancement Cavities . . . . . . . . . 74

5.4 Other Experiments with High-Power Enhancement Cavities . . . . . . . . 76

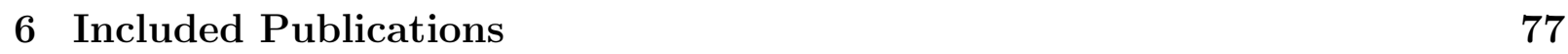

\begin{tabular}{lll}
\hline & Appendix & 125
\end{tabular}

$7.1 \quad$ Experiments Involving Enhancement Cavities . . . . . . . . . . . . . . . . 125

7.2 An Example of Comb-Cavity Detuning . . . . . . . . . . . . . . . . . . . . 126

7.3 Overview of XUV Output Coupling Methods . . . . . . . . . . . . . . . . . 127

7.4 Data Archiving . . . . . . . . . . . . . . . . . . . . . 130

\begin{tabular}{ll}
\hline Bibliography & 133
\end{tabular}

\begin{tabular}{|l|l}
\hline Acknowledgements & 144
\end{tabular}

\begin{tabular}{lr}
\hline Curriculum Vitae & 146
\end{tabular}

\begin{tabular}{lr}
\hline Publications 2007-2011 & 147
\end{tabular} 


\section{Zusammenfassung}

Im Rahmen dieser Arbeit wurde ein Femtosekunden Überhöhungsresonator (ÜR) entwickelt und die Skalierung dieser Technologie zu hohen Leistungen untersucht. Der ÜR wurde zur Erzeugung kohärenter extrem ultravioletter (XUV) Strahlung mittels Generation hoher Harmonischer (HHG) in einem Gas als nichtlineares Medium eingesetzt.

ÜR sind optische Resonatoren, in welchen kontinuierliche Laserstrahlung oder Pulse eines Frequenzkamms kohärent überlagert werden. Im Falle des Frequenzkamms entsteht im Resonator ein leistungsstarker Puls, der mit der Repetitionsrate des Eingangspulszuges (mehrere zehn $\mathrm{MHz}$ ) zirkuliert. Die Leistungsüberhöhung, die durch die Verluste und die Dispersion des Resonators beschränkt ist, beträgt üblicherweise bis zu drei Größenordnungen. Somit sind ÜR ideal zum Treiben nichtlinearer Prozesse mit niedrigen Konversionseffizienzen geeignet, da das nicht konvertierte Licht nach jeder Interaktion mit dem nichtlinearen Medium wiederbenutzt wird, was hohe Intensitäten im Resonator aufrechterhält. ÜR, gekoppelt an leistungsstarke Laserquellen, die Pulse mit multi-MHz Repetitionsraten im Sichtbaren und ins Infrarote emittieren, bieten die Aussicht, diese Strahlung mit hohem Wirkungsgrad in Regionen des elektromagnetischen Spektrums zu konvertieren, in denen Laserübergänge entweder selten oder ineffizient sind. Insbesondere wurde die Entwicklung von ÜR im letzten Jahrzehnt durch die Aussicht auf kompakte, leistungsstarke köhärente Strahlungsquellen für das Vakuum UV, XUV und weiche Röntgenstrahlung mittels HHG, sowie für das mittlere und ferne Infrarot mittels Differenzfrequenzmischung motiviert. Beim gegenwärtigen Stand der Entwicklung sind allerdings die fundamentalen Grenzen dieser Technologie bei weitem nicht erreicht.

Der in dieser Arbeit vorgestellte ÜR wird mit einem Yb-basierten Lasersystem mit einer Repetitionsrate von $78 \mathrm{MHz}$ angeregt und unterstützt Pulsedauern von $200 \mathrm{fs}$ und $2 \mathrm{ps}$ bei Durchschnittsleistungen von $20 \mathrm{~kW}$ bzw. $72 \mathrm{~kW}$, begrenzt durch intensitätsbedingte Effekte in den Spiegeln. Bis heute ist dies das höchste Leistungsniveau, welches mit ultrakurzen Pulsen bei einer multi-MHz Repetitionsrate erreicht wurde und es entspricht einer Steigerung von etwa einer Größenordnung gegenüber dem Stand der Entwicklung am Anfang dieser Arbeit in 2007. Weiterhin wurde die erste hochsensible Messmethode für die Umlaufdispersion in einem ÜR entwickelt, die Messungen bei voller Leistung erlaubt. Damit wurden die intensitätsbedingten Limitierungen der Leistungsskalierung bestätigt.

Eine der größten Herausforderungen der ÜR-gestützten XUV-Erzeugung ist die Auskopplung der im Resonator erzeugten hohen Harmonischen. Als Teil dieser Arbeit wurden neue Methoden entwickelt, die die Aussicht auf eine Erweiterung der aktuellen Limitierungen bezüglich Leistungsskalierung und Auskoppeleffizienz bieten. Erste HHG Experimente wurden durchgeführt und eine gute Übereinstimmung mit der Theorie wurde festgestellt.

Diese Ergebnisse stellen Meilensteine dar, auf dem Weg zu leistungsstarken, kompakten Strahlungsquellen für Spektralbereiche in denen Anwendungen bisher nur mit Synchrotrons oder Freie-Elektronen-Lasern demonstriert wurden. Zusätzlich zur Kompaktheit bietet die Strahlungserzeugung in ÜR den Vorteil ultrakurzer Pulse bei hohen Repetitionsraten. Dies eröffnet neue Möglichkeiten für die Grundlagenforschung, insbesondere in der Attosekundenphysik und der Frequenzkammspektroskopie. 


\section{Abstract}

In the course of this work, a femtosecond enhancement cavity (EC) was developed and scaling the intracavity power to very high values was investigated. The EC was then used for the generation of coherent extreme ultraviolet (XUV) radiation via high-harmonic generation (HHG) in a gas as a nonlinear medium.

EC's are passive optical resonators in which continuous-wave laser radiation or pulses of a frequency comb are coherently overlapped. In the case of a frequency comb this results in one (or several) high-power pulse(s), circulating in the resonator with the repetition rate of the initial pulse train of typically several tens of $\mathrm{MHz}$. The enhancement, which is limited by the cavity losses and chromatic dispersion, usually amounts to a few orders of magnitude. This makes EC's ideally suited to boost the efficiency of nonlinear processes with low single-pass conversion efficiency since the non-converted light is recycled after each interaction with the nonlinear medium, thus sustaining high intracavity intensities. EC's coupled to high-power laser sources emitting multi-MHz-repetition-rate, phase-stabilized femtosecond pulses, in the visible and infrared promise to efficiently convert the radiation of these sources to regions of the electromagnetic spectrum, where direct laser action is either scarce, inefficient, or not available at all. In particular, the prospect of table-top sources of bright, coherent radiation in the Vacuum UV, XUV and soft X-ray region, as well as in the mid and far-infrared, via HHG and difference frequency mixing, respectively, has been motivating the development of EC's for the last decade. But still, fundamental limitations of EC's are yet far from being reached.

The EC presented in this thesis is seeded by a $78-\mathrm{MHz}$ repetition rate Yb-based laser system and supports pulse durations of $200 \mathrm{fs}$ and $2 \mathrm{ps}$ with average powers of $20 \mathrm{~kW}$ and $72 \mathrm{~kW}$, respectively, limited by intensity-related effects in the mirrors. To the best of our knowledge, this power level is the highest for ultrashort pulses at a multi- $\mathrm{MHz}$ repetition rate reported to this day and corresponds to an increase of roughly one order of magnitude with respect to the state of the art at the beginning of this work in 2007. Furthermore, we developed the first highly sensitive intracavity dispersion measurement method working for fully loaded cavities, in particular including nonlinear processes. With this method the intensity-related power scaling limitations were confirmed.

One of the main challenges of high-power EC-based XUV generation is coupling out the intracavity generated high harmonics from the EC. Novel methods were developed as a part of this work, offering the prospect of pushing the limits of high-power operation of current output couplers and promising an increased output coupling efficiency. Finally, first HHG experiments were carried out successfully and good agreement with the theoretical prediction was obtained.

These results represent milestones on the way to realizing bright, table-top radiation sources for spectral regions in which applications have so far only been demonstrated with synchrotron or free-electron laser radiation. In addition to the simplicity and compactness of the setup, laser-based radiation generation exhibits the advantage of ultrashort pulses at high repetition rates, opening the door to new insights in fundamental science, in particular in attosecond physics and frequency comb spectroscopy. 
Jedes Wekzeug trägt den Geist in sich, aus dem heraus es geschaffen worden ist.

Werner Heisenberg

\section{Chapter 1}

\section{Introduction}

\subsection{About Light: From the Genesis to the Laser}

Light is present in virtually all areas of life. It is involved in the majority of processes in nature, it plays a primordial role in most religions and it has been one of the fundamental (and perhaps the oldest) subjects of philosophy, arts and scientific research. The preoccupation with light and in particular, with the study of its origin and properties and with its control has always constituted an integral part of culture ${ }^{1}$.

Many principles of optics, i.e. the science of light, were already known in antiquity. Euclid's description of the principles of geometrical optics from the $3^{\text {rd }}$ century BC counts among the first documented scientific works in optics. The Greek geometrician possessed the mathematical apparatus necessary for the description of fundamental facts related to the rectilinear propagation of light. Archimedes, who lived around the same time as Euclid, is said to have defended Syracuse from the invading Roman fleet by using large mirrors to focus the sunlight on the enemy ships, setting them on fire. Another testimony of the knowledge in the field of optics during antiquity is found in a book of Ptolemy from the $2^{\text {nd }}$ century AD. In addition to his predecessor's work, he describes the phenomenon of refraction of light at interfaces between media of different optical densities.

The most important treaty on optics during the Middle Ages was written by Ibn alHaitham (or Alhazen) around the year 1000. He delivers descriptions of the magnification of lenses, spherical aberration, parabolic mirrors and explanations for phenomena such as the formation of rainbows and atmospherical refraction. Together with the writings of Ptolemy, this work represents the fundamental reference on optics up to the $17^{\text {th }}$ century. After this, the field of optics experienced rapid development, supported by the refinement of the manufacturing of lenses and measurement devices and techniques as well as by the evolution of mathematical tools. Outstanding landmarks of this development include the observation of the interference of light by R. Boyle and R. Hooke and the foundation of the wave theory for light by $\mathrm{Ch}$. Huygens in the $17^{\text {th }}$ century, experiments on interference and diffraction by Th. Young, the discovery of the polarization of light by L. Malus, detailed explanations

\footnotetext{
${ }^{1}$ The following historical review is largely based on Th. and H. Walther's book Was ist Licht? 1 .
} 
for the interference and diffraction phenomena by Fresnel and Fraunhofer in the early $19^{\text {th }}$ century and the direct measurement of the speed of light by L. Foucault and H. Fizeau in 1850. In the late $19^{\text {th }}$ century, J. C. Maxwell succeeded in mathematically describing the experiments on the generation of variable electric and magnetic fields initiated 40 years earlier by M. Faraday. The speed of the electromagnetic wave propagation predicted by Maxwell's equations coincided with the previously measured speed of light. Experiments performed by H. Hertz in 1888 demonstrated electromagnetic waves directly, rounding up the classical wave picture and providing an apparent conclusion of the research on the nature and the properties of light.

However, the understanding of light-matter interaction provided by the classical wave model of light fails at describing atomic absorption and emission processes. The necessary expansion of the existing theory was provided by Max Planck in 1900 with the introduction of the quantum hypothesis. Planck postulated that an electronically oscillating system can only exchange energy of discrete amounts with an electromagnetic field. The energy $E$ of a quantum of radiation is proportional to the frequency $f$ of the radiation via $E=h f$, where Planck's constant $h$ is a new fundamental physical constant. Einstein's explanation of the photoelectric effect in 1905 provided the validation of the new model, using particles of light 2 , later called photons. Shortly thereafter, the hypothesis of a coexistence of waves and particles was extended to electrons by L. de Broglie and supported by the observation of interference phenomena of electrons at crystal lattices by C. Davisson and L. Germer.

The wave-particle duality marks modern physics and, in particular, the field of quantum electronics, which studies quantum phenomena related to the interaction of electromagnetic radiation with matter. The process underlying the probably most important sources of radiation in this field is amplification by stimulated emission of radiation, which was first predicted and experimentally demonstrated for microwaves (therefore the acronym maser) in the first half of the last century, and then extended to the infrared, visible, ultraviolet and even X-ray regions of the electromagnetic spectrum, loosely designated as light, conferring the acronym laser ${ }^{3}$. The output signal of this coherent amplification process reproduces the frequency of the input electromagnetic wave with the respective phase, but with a substantially increased amplitude. In the case of the laser, the gain medium consists of a quantum-mechanical system such as a collection of atoms, molecules or ions or a semiconducting crystal, which is excited by a pumping process (providing the energy necessary for the amplification process) into higher energy levels, so that a population inversion is achieved In conjunction with the feedback provided by an (optical) resonator, the radiation obtained with this amplification mechanism forms a bright beam with unequaled spatial and temporal coherence properties.

\footnotetext{
${ }^{2}$ In the second half of the $17^{\text {th }}$ century, Newton developed a particle-based model for light. However, his theory lost ground to Huygens' wave theory, which could explain most of the experiments of that time.

${ }^{3}$ During the time of this doctorate work, the scientific community celebrated the 50 years anniversary of the first experimental demonstration of the laser by Th. H. Maiman in 1960.

${ }^{4} \mathrm{~A}$ detailed description of the mode of operation of the laser would exceed the frame of this section. A textbook introduction is given in A. Siegman's book Lasers [2].
} 


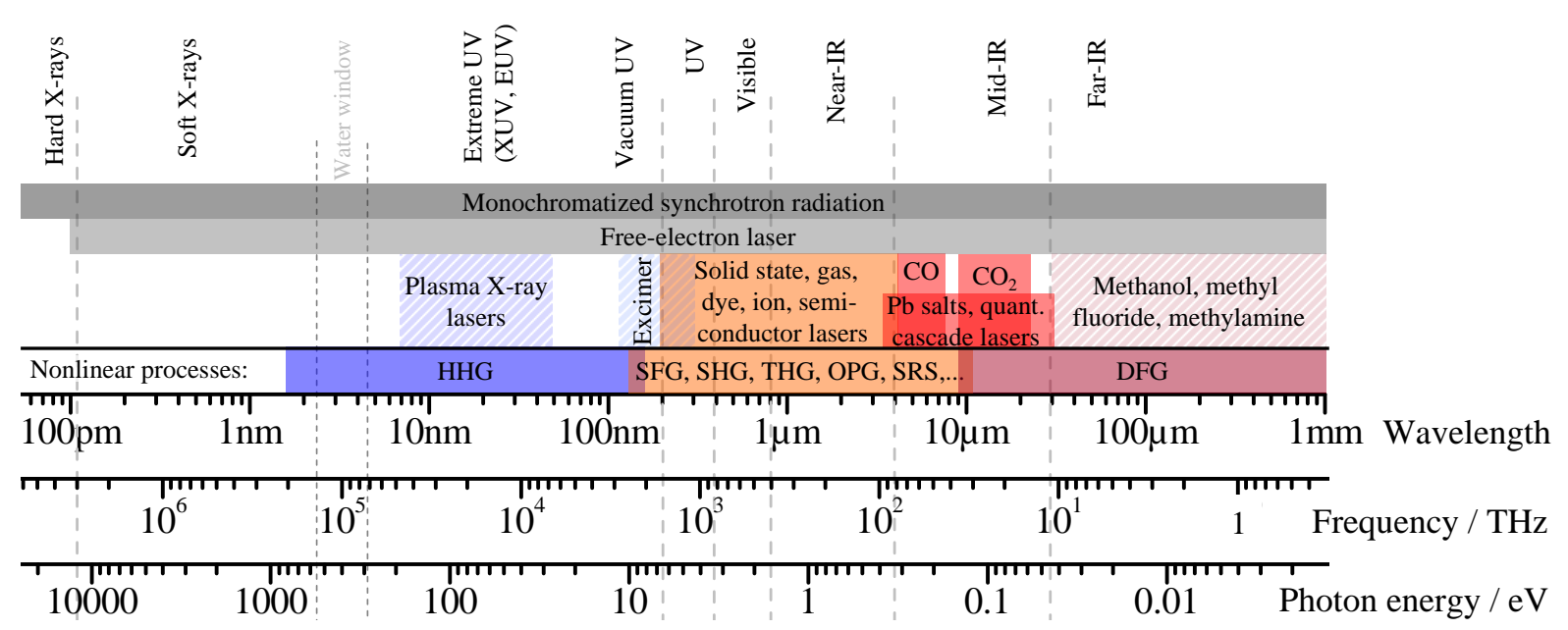

Figure 1.1: Coherent light sources. Hashed regions: only scarce, discrete laser lines (transitions) are available. Main nonlinear processes used to convert laser light: SHG, THG, HHG: second-, third- and high-harmonic generation, SFG, DFG: sum- and difference-frequency generation, OPG: optical parametric generation, SRS: stimulated Raman scattering.

The past few decades have witnessed a rapid development of laser sources, motivated by and enabling myriads of applications ranging from the investigation of microcosm dynamics over telecommunications to industrial manufacturing. Today, a great variety of gain media is available, providing quantum energy transitions for laser action over large parts of the electromagnetic spectrum between X-rays and far-infrared. Still, significant spectral regions remain, where direct laser action is either inefficient or not available at all, other than by means of large facilities such as free-electron lasers. Figure 1.1 provides an overview of the available coherent light sources, compiled using data from [3 6]. Most notably, the spectral regions below $200 \mathrm{~nm}$ (Vacuum UV, XUV, soft X-rays) and above $10 \mu \mathrm{m}$ (mid- and far-IR) exhibit a sparse presence of efficient direct laser action. A convenient alternative of generating high-power coherent radiation in these spectral regions relies on the conversion of light emitted by a laser in a different spectral region via nonlinear processes. However, these processes can exhibit low conversion efficiencies and in general, their efficiency increases with increasing intensity. In the sixties, shortly after the first observation of an optical nonlinear process - namely second-harmonic generation (SHG) - the technique of passive laser light enhancement in an optical resonator was successfully employed to improve its overall conversion efficiency. In recent years, this technique has gained new importance due to the advent of high-power laser sources emitting phase-stabilized femtosecond pulses at multi- $\mathrm{MHz}$ repetition rates, in conjunction with advances in mirror coating technology enabling the construction of high-finesse broadband resonators. Today, the generation of radiation via nonlinear processes driven by femtosecond pulses, which are enhanced in passive resonators, exhibits the potential of approaching power levels necessary for applications which so far have only been demonstrated with synchrotron or free-electron laser radiation. In addition, the laser-based generation provides the prospect of unprecedented time and space coherence in the envisaged regions of the electromagnetic spectrum 


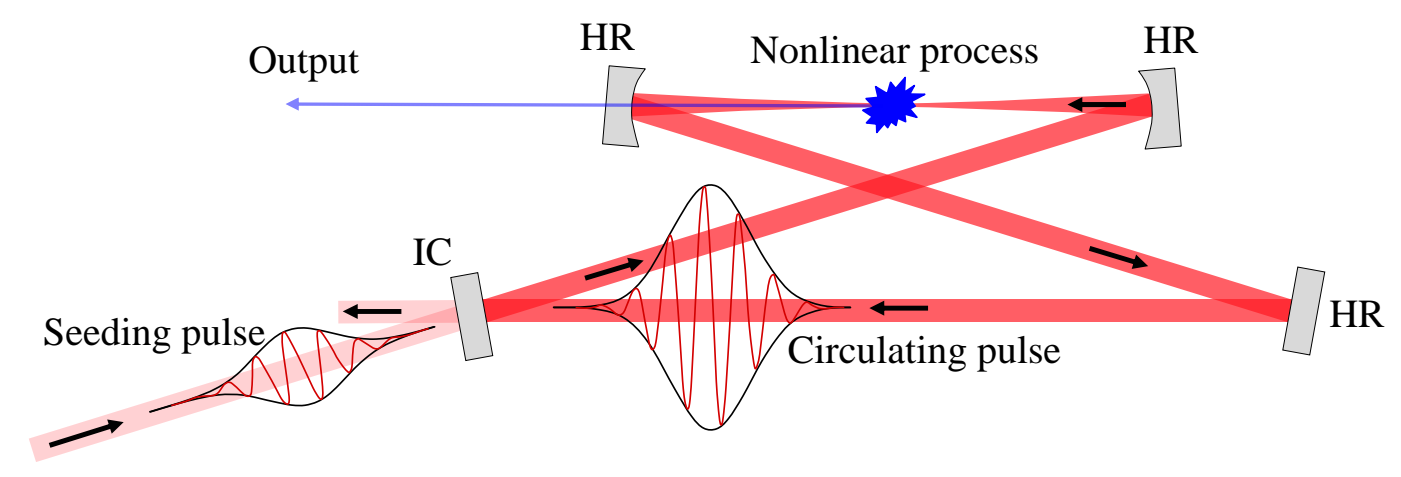

Figure 1.2: Schematic of an enhancement cavity. IC: input coupler, HR: highly reflective mirror (for the fundamental radiation).

and exhibits the advantages of simplicity, compactness and low cost. Further development of the femtosecond enhancement cavity technology has motivated the research reported in this thesis.

\subsection{Motivation: Enhancement of Nonlinear Processes in Passive Optical Resonators}

An enhancement cavity (EC) is a passive optical resonator in which laser light is coherently overlapped. Coherence in this context refers to the condition that the input laser light has a constant phase relationship with the light "stored" in the EC, i.e. the intracavity light ${ }^{5}$.

Figure 1.2 shows a schematic of an EC. A train of input (also referred to as seeding) laser pulses, equidistant in time and with a fixed electric field phase relation, hits the input coupler of the EC. Under proper conditions, a high-power pulse forms in the cavity, circulating at the repetition rate of the initial pulse train (usually several tens of $\mathrm{MHz}$ ), and receiving energy from each of the seeding pulses ${ }^{6}$. The power enhancement in an EC is limited by the cavity losses and chromatic dispersion, and in state-of-the-art setups it amounts to a few orders of magnitude. EC's are ideally suited to boost the efficiency of nonlinear processes with low single-pass conversion efficiency (such as HHG or DFG) because firstly, the low conversion efficiency does not affect the enhancement considerably, enabling high intracavity intensities and secondly, the pulse used to drive the nonlinear process is recycled after each interaction with the nonlinear medium 7 .

\footnotetext{
${ }^{5}$ This can be either satisfied by a single-frequency continuous-wave (CW) laser, or by a laser emitting several frequencies with a fixed phase relationship, which is the case of a mode-locked laser generating pulses. Since CW operation can be regarded as a special case of a mode-locked laser, the following discussion of pulsed EC's includes the CW case.

${ }^{6}$ Note that multiple pulses can be stored in the EC in the same manner, if the cavity roundtrip time is a multiple of the pulse repetition period.

${ }^{7}$ The task of coupling out the new frequencies from the cavity strongly depends on the participating wavelengths.
} 


\subsection{Motivation: Enhancement of Nonlinear Processes in Passive Optical Resonators}

EC's are often referred to as external resonators because they do not contain a gain medium. Driving nonlinear processes in such a resonator rather than in a laser cavity exhibits several additional advantages. The lack of elements prone to the high average and peak powers (such as the gain medium and/or the mode-locking components) circumvents their inherent limitations related to thermal and nonlinear effects and also facilitates operation under vacuum conditions, which is mandatory for certain applications. Moreover, the input laser light can be readily subjected to single-pass processes (such as amplification, nonlinear spectral broadening, pulse picking, pulse shaping etc.) before seeding the EC. Further advantages include the flexibility concerning the intracavity power regime which can be easily varied over a large range by adjusting the parameters of the seeding pulses, an outstanding intracavity beam quality and the action of the passive resonator as a high-frequency low-pass noise filter. The on-resonance sensitivity amplification of singleroundtrip dispersion and losses can also be used for accurate detection measurements.

The principle of resonant enhancement of an optical nonlinear process was demonstrated as early as in 1966 for SHG with a continuous-wave (CW) laser [7]. Further development followed, see [8 10] and references therein. Other EC's were constructed for pulsed SHG [11, 12], third harmonic generation of a CW laser [13], frequency mixing of two CW lasers [14], the generation of $\mathrm{THz}$ radiation via DFG 15$]$ and recently, an EC-based optical parametric chirped-pulse amplification scheme was proposed [16]. First proofs of principle for ECbased HHG in a neutral noble gas jet have attracted high interest in recent years 17,238 . However, the power scaling limitations of these unique sources are still far from being reached.

At our institute there are two main motivations of pursuing further power scaling of high-repetition rate HHG in EC's. On the one hand, this technology offers the prospect of extreme nonlinear optics and in particular, isolated attosecond pulse generation at repetition rates of several tens of $\mathrm{MHz}$, which is a few orders of magnitude larger than in current systems 24,25$]$. This would open new perspectives in attosecond physics, allowing for new insights to both the collective and individual motion of electrons on atomic and molecular scales. On the other hand, efficient XUV generation with high repetition rates will enable high-precision frequency comb spectroscopy in a hitherto inaccessible spectral region [17]. In the frequency domain, the distance between the comb lines equals the repetition frequency of the ultrashort pulses and is transferred to the generated high harmonic spectrum. Fewer comb lines per unit of frequency implies more energy per comb line and the possibility of resolving single lines.

The significance of investigations related to the power scaling of the femtosecond enhancement cavity technique extends beyond these two particular applications. The availability of a table-top source of bright VUV and XUV radiation would undoubtedly result in a significant boost of pertinent experiments in numerous fields, including XUV microscopy and tomography [26], interferometry and holography [27], plasma physics [28], spectroscopy [29], at-wavelength-testing of components for EUV lithography [30] and sur-

\footnotetext{
${ }^{8} \mathrm{~A}$ more detailed overview of EC's for HHG is given in Appendix 7.1 .
} 
face and material studies [31], just to name the most prominent ones. So far, some of these applications have only been demonstrated using synchrotron radiation. Besides HHG in a gas, the study and development of power scaling of the EC technology can be useful for arbitrary nonlinear processes. Therefore, the motivation of this work ties in with the basic preoccupation with the study of light, mentioned at the beginning of this chapter.

\subsection{Overview of the Results}

During the past four years, in the frame of this doctorate work a femtosecond enhancement cavity experiment was set up and investigations concerning the power scaling of this technology were performed. The results can be summed up into 4 categories:

1 - Power scaling of the empty cavity. The EC presented in this thesis is seeded by an Yb-based laser system and supports 200 -fs pulses with $20 \mathrm{~kW}$ of average power at a repetition rate of $78 \mathrm{MHz}$. Beyond this power level, intensity-related effects in the mirrors were identified, leading to resonator instabilities and damage. By extending the pulse duration to 2 ps by chirping the seed laser pulses, we could obtain $72 \mathrm{~kW}$ of intracavity circulating power with the maximum available input power of $50 \mathrm{~W}$. To the best of our knowledge, this power level is the highest for ultrashort pulses at a multi-MHz repetition rate reported in the literature to this day and corresponds to an increase of roughly one order of magnitude with respect to the state of the art at the beginning of this work [32. The limitations identified spawned the design of the next-generation EC in our group, following the strategy of increasing the spot size on the mirrors.

2 - Advanced diagnostics. The first highly sensitive intracavity dispersion measurement method working at high intra-cavity intensities was developed, based on spatially and spectrally resolved interferometry (SSI) of a copy of the input beam to the cavity with a copy of the circulating beam. In particular, dispersion measurements of the empty cavity have confirmed the intensity-related power scaling limitations. This method is expected to be instrumental to the optimization of the operation of EC's including nonlinear processes.

3 - $\boldsymbol{X} \boldsymbol{U} \boldsymbol{V}$ output coupling. One of the main challenges of EC-based XUV generation is coupling out the intracavity generated high harmonic radiation from the EC, especially at the power levels demonstrated in our EC. As a part of this work, the suitability of several output coupling methods for high-power operation was investigated. Two novel methods were developed, offering the prospect of pushing the limits of high-power operation and efficient output coupling of the shortest wavelengths.

4 - High-harmonic generation. First HHG experiments were carried out successfully at a moderate power level. With $200 \mathrm{fs}$ pulse duration, $2.4 \mathrm{~kW}$ of average power and a focus radius $w_{0}=23 \mu \mathrm{m}\left(1 / e^{2}\right.$-intensity decay), harmonics up to order 17 generated in Xe were detected. The results are in excellent agreement with theoretical predictions. These experiments are intended as benchmarks for future scaling of the HHG process, especially employing one of the novel output coupling methods mentioned above, suited for the power regimes which have been demonstrated in the empty cavity.

\footnotetext{
${ }^{9} \mathrm{~A}$ number of further exciting applications are summarized in Refs. 4.5.
} 
In the following, an overview of the journal publications related to the results in these 4 categories, which I co-authored, is given:

1 - Power scaling of the empty cavity. The main results are published in the following two papers, for which I performed the majority of the experimental work and manuscript preparation:

- I. Pupeza, T. Eidam, J. Rauschenberger, B. Bernhardt, A. Ozawa, E. Fill, A. Apolonski, Th. Udem, J. Limpert, Z. A. Alahmed, A. M. Azzeer, A. Tünnermann, T. W. Hänsch and F. Krausz, "Power scaling of a high repetition rate enhancement cavity," Opt. Letters 12, 2052 (2010).

- I. Pupeza, T. Eidam, J. Kaster, B. Bernhardt, J. Rauschenberger, A. Ozawa, E. Fill, T. Udem, M. F. Kling, J. Limpert, Z. A. Alahmed, A. M. Azzeer, A. Tünnermann, T. W. Hänsch, and F. Krausz, "Power scaling of femtosecond enhancement cavities and high-power applications," Proc. SPIE 7914, 79141I (2011).

In the frame of the collaboration with the Hänsch-group, I contributed (mainly with discussions) to the green-cavity project led by Birgitta Bernhardt. The following manuscript is currently in preparation:

- B. Bernhardt, A. Ozawa, A. Vernaleken, I. Pupeza, J. Kaster, Y. Kobayashi, R. Holzwarth, E. Fill, F. Krausz, T. W. Hänsch, and Th. Udem, "First green enhancement cavity generating an ultra violet frequency comb," manuscript in preparation.

2 - Advanced diagnostics. The dispersion measurement method is published in the following manuscript. While I contributed most of the experimental work, data interpretation and manuscript preparation, the initial idea of applying SSI as well as great support with the experiment and data interpretation came from Xun $\mathrm{Gu}$, along with substantial support from the other authors:

- I. Pupeza, X. Gu, E. Fill, T. Eidam, J. Limpert, A. Tünnermann, F. Krausz, and T. Udem, "Highly Sensitive Dispersion Measurement of a High-Power Passive Optical Resonator Using Spatial-Spectral Interferometry," Opt. Express 18, 26184 (2010).

3 - $\boldsymbol{X} \boldsymbol{U} \boldsymbol{V}$ output coupling. For the following paper I performed the majority of the experimental work and manuscript preparation:

- I. Pupeza, E. Fill, and F. Krausz, "Low-loss VIS/IR-XUV beam splitter for highpower applications," Opt. Express 19, 12108 (2011).

For the following paper, I contributed mainly the experimental setup and the measurements described in Section 4, together with Jan Kaster, and helped with the manuscript preparation:

- Y.-Y. Yang, F. Süssmann, S. Zherebtsov, I. Pupeza, J. Kaster, D. Lehr, E.-B. Kley, E. Fill, X.-M. Duan, Z.-S. Zhao, F. Krausz, S. Stebbings, and M. F. Kling, "Optimization and characterization of a highly-efficient diffraction nanograting for $\mathrm{MHz}$ XUV pulses," Opt. Express 19, 1955 (2011). 
For the following two publications I performed the majority of the experimental work and contributed to the theory and manuscript preparation:

- J. Weitenberg, P. Russbüldt, T. Eidam, and I. Pupeza, "Transverse mode tailoring in a quasi-imaging high-finesse femtosecond enhancement cavity," Opt. Express 19, 9551 (2011).

- J. Weitenberg, P. Russbüldt, I. Pupeza, T. Udem, H.-D. Hoffmann, and R. Poprawe, "Geometrical on-axis access to high-finesse resonators by quasi-imaging," manuscript in preparation.

4-High-harmonic generation. The HHG results reported in this thesis are preliminary and have not yet been published. However, they are very promising and a timely publication is being considered.

\subsection{Structure of the Thesis}

The subsequent chapters are organized as follows:

- Chapter 2 reviews the most relevant theoretical aspects of the area of this work, including the fundamentals of mode-locked lasers, ultrashort pulses, enhancement cavities and high-harmonic generation (HHG).

- Chapter 3 addresses the objectives of the experiments presented in this thesis and the technological challenges on the way to reaching them. An overview of the solutions worked out during this thesis and of the remaining challenges is given.

- Chapter 4 presents the experimental results elaborated during this work.

- Chapter 5 gives an outlook on future research motivated by and related to the results of this work.

- Chapter 6 contains the journal articles [33 37] constituting the cumulative part of this thesis as well as a description of the author's contribution to these publications. The results contained in these papers are not repeated in Chapters 1 to 5 in detail. Rather, these results are referred to throughout Chapters 1 to 5 with standard citations, followed by the remark "see Chapter 6]' 


\section{Chapter 2}

\section{Theoretical Background}

\subsection{Passive Enhancement of Ultrashort Pulses}

The setup of any femtosecond enhancement cavity (EC) experiment consists of three main components. Firstly, the pulses are generated by a mode-locked oscillator and optionally post-processed (e.g. amplified and/or spectrally broadened and temporally compressed etc.). The second component is the EC in which the laser pulses are overlapped coherently. If the EC is used for nonlinear conversion, it also contains the respective mechanism. And thirdly, one or more feedback loops are needed to ensure an interferometric overlap of the seeding laser pulses with the pulse(s) circulating in the enhancement cavity.

Ideally, a steady state is aimed for in which the enhanced electric field circulating in the passive cavity is a power scaled version of the seeding laser field. However, due to the fact that the spectrum emitted by a mode-locked laser consists of equidistant modes and the resonances of a passive cavity are in general not equidistant in the frequency domain, this task is highly challenging. To explain this challenge, we provide a theoretical background of the three main components. The different nature of the mechanisms underlying the generation of the pulses and their passive enhancement is addressed, and aspects particularly relevant for the experimental results presented in this thesis are emphasized.

\subsubsection{Ultrashort Pulses from Mode-Locked Lasers}

Mode-locking underlies the generation of the shortest light pulses directly from a laser oscillator. The advent of self-mode-locking techniques (see. e.g. [24]) merely 20 years ago and the subsequent development of phase stabilization in the last decade have had a significant impact on scientific research and technology. With this technique, light pulses comprising only a few oscillations of the electric field can be generated, confining the available laser energy to pulse durations on a femtosecond timescale. In addition, phase stabilization guarantees the pulse-to-pulse reproducibility of the electric field within the pulse intensity envelope. Pulses with these characteristics have marked the birth of ultrafast and attosecond science [24,25], studying and controlling light-matter interactions at unprecedented intensity levels and atomic dynamics on previously inaccessible time scales. Phase-stabilized 
(a) a single mode :

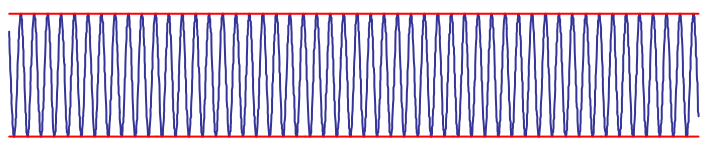

(b) 3 modes :

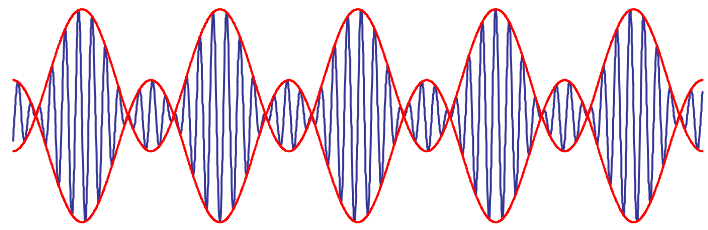

(c) 7 modes :

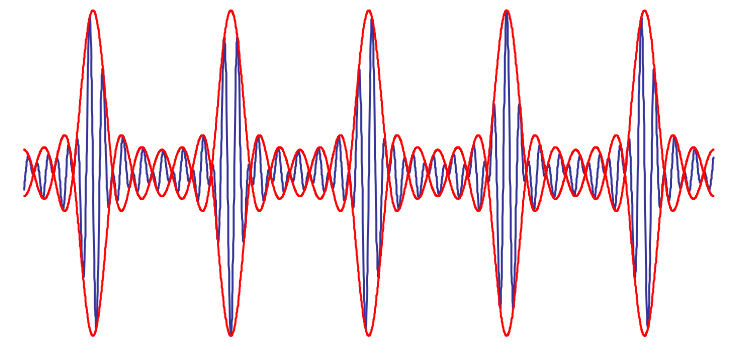

Figure 2.1: The formation of ultrashort pulses: superposition of axial modes of an ideal Fabry-Perot resonator, oscillating with the same phase. The red line indicates the intensity profile for (a) a single mode, (b) for 3 and (c) for 7 modes.

mode-locked lasers have also enabled high-precision optical frequency metrology. Their frequency spectrum is a regular structure of equidistant lines, called frequency comb, see e.g. 38 43]. The frequency comb can be parameterized by using only two radio frequencies $(\mathrm{RF})$, which can be easily measured, e.g. with respect to the hyperfine transition of cesium at $9.193 \mathrm{GHz}$ which provides the standard for one second. This constitutes a bridge transferring the measurement precision over the frequency gap between the RF spectrum and optical frequencies. A textbook-level description of the mode of operation of mode-locked lasers can be found e.g. in [4] or [45]. Here, we restrict the description to an overview of the basic mechanisms leading to the formation of a train of femtosecond pulses with a fixed phase relationship.

Mode locking refers to imposing a certain, fixed phase relation to the resonant axial (longitudinal) modes of a laser cavity. Figure 2.1 visualizes the intensity of (a) a single mode, i.e. continuous-wave operation, and the superposition of (b) 3 and (c) 7 modes of an ideal Fabry-Perot resonator, if these modes oscillate with identical phases. At a fixed time, or at a fixed space, the modes interfere constructively at equidistant maxima in space, or in time, respectively, which leads to the formation of light pulses. The more modes simultaneously resonant in the cavity, i.e. the broader the bandwidth of contributing frequencies, the shorter the resulting pulses. However, in a real laser cavity, the circulating light pulses are subjected to dispersion, i.e. to a nonlinear dependence of the wavenumber $k(\omega)$ on the angular frequency $\omega$. In general, for a pulse with a spectrum centered around the frequency $\omega_{\mathrm{c}}$, the wavenumber $k(\omega)$ can be expanded in a Taylor series:

$$
k(\omega)=\frac{\omega_{\mathrm{c}}}{v_{\mathrm{ph}}}+\frac{\omega-\omega_{\mathrm{c}}}{v_{\mathrm{gr}}}+\frac{1}{2} G V D\left(\omega-\omega_{\mathrm{c}}\right)^{2}+\ldots,
$$


where $v_{\mathrm{ph}}, v_{\mathrm{gr}}$ and GVD denote the phase velocity of propagation of the frequency component $\omega_{\mathrm{c}}$, the group velocity of the pulse and the group velocity dispersion, respectively (cf. e.g. Section 9.1 in $[2]$ ). The terms of orders 2 (i.e. GVD) and higher describe the dispersion accumulated by the pulse upon propagation and thus, the pulse lengthening in time. In the presence of non-zero net roundtrip dispersion, a pulse circulating in the laser cavity would broaden infinitely in time. In a mode-locked laser, however, the pulse reproduces after each round trip, which is equivalent to the fact that the effect of residual cavity dispersion on the pulse is compensated for upon each roundtrip. The intensity evolution of the pulse is given by the relative phase of the frequency components. Therefore, the nonlinear process of self phase modulation (SPM) can be used to perform this compensation and acts as a simultaneous switch for all active modes. The effect of SPM upon a roundtrip is determined by the interplay of the intracavity nonlinear mechanisms, such as the amplification and propagation in the active medium and (usually) an additional mode-locking mechanism. Dimensioning the phase effects in a mode-locked oscillator can be done by solving the nonlinear Schrödinger equation, see e.g. Section 10.3 in [2]. The interplay between the cavity dispersion, the mode-locking mechanism and the active material net gain bandwidth also determines the (achievable) output pulse duration of a mode-locked laser. A mathematical model of the mode-locking process can be found e.g. in [46, 47]. A decisive result is that the mode locking mechanism counterbalances the terms of order 2 and higher in the Taylor expansion in eq. (2.1), implying:

$$
k(\omega)=\frac{\omega_{\mathrm{c}}}{v_{\mathrm{ph}}}+\frac{\omega-\omega_{\mathrm{c}}}{v_{\mathrm{gr}}} .
$$

This means in particular that the pulse can propagate with a different group velocity $v_{\mathrm{gr}}$ than the phase velocity $v_{\mathrm{ph}}$ of the wave with frequency $\omega_{\mathrm{c}}$. The following derivation will show that this fact is of crucial importance for the control of the electric field of the generated pulse train.

The steady-state resonance boundary condition of a linear Fabry-Perot oscillator with length $L$ (or $2 L$ for a ring cavity) is given by:

$$
2 k\left(\omega_{N}\right) L=2 \pi N,
$$

where the integer $N$ denotes the axial (longitudinal) mode number. The last two equations determine the frequencies of the modes of a mode-locked oscillator:

$$
\omega_{N}=N \frac{2 \pi}{2 L} v_{\mathrm{gr}}+\omega_{\mathrm{c}}\left(1-\frac{v_{\mathrm{gr}}}{v_{\mathrm{ph}}}\right) .
$$

Thus, the frequency difference between two adjacent modes amounts to:

$$
\omega_{N+1}-\omega_{N}=\frac{2 \pi}{2 L} v_{\mathrm{gr}}=: \omega_{\mathrm{r}}
$$

and corresponds to $2 \pi$ times the repetition frequency of the pulses, which we will denote by $\omega_{\mathrm{r}}$. 


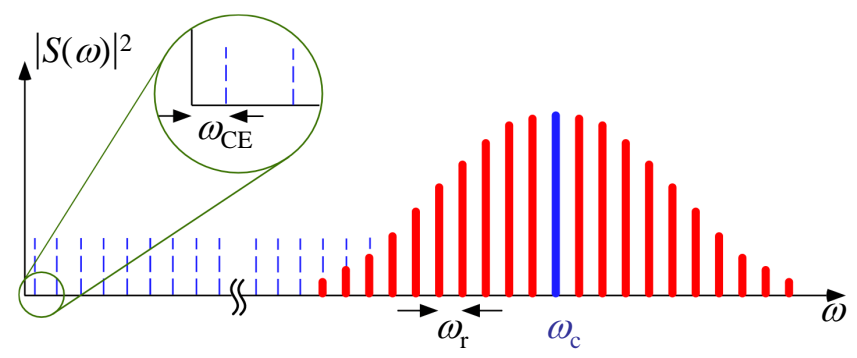

Figure 2.2: Frequency-domain representation of the frequency comb emitted by a phasestabilized mode-locked laser (as in 40]). The comb parameters are: the repetition frequency $\omega_{\mathrm{r}}$ and the carrier-envelope frequency $\omega_{\mathrm{CE}}$.

The second term on the right-hand side of eq. (2.4) represents an offset frequency, which we will denote by $\omega_{\mathrm{CE}}$ :

$$
\omega_{\mathrm{CE}}:=\omega_{\mathrm{c}}\left(1-\frac{v_{\mathrm{gr}}}{v_{\mathrm{ph}}}\right) .
$$

The abbreviation CE stands for carrier-envelope due to the significance of $\omega_{\mathrm{CE}}$ in the time domain, which we will address shortly. In conclusion, the spectrum emitted by a stable mode-locked laser is a comb of equidistant modes with a spacing $\omega_{\mathrm{r}}$ and offset from a multiple of $\omega_{\mathrm{r}}$ by $\omega_{\mathrm{CE}}$ :

$$
\omega_{N}=N \omega_{\mathrm{r}}+\omega_{\mathrm{CE}} .
$$

Figure 2.2 shows such a spectrum. With a spectral amplitude envelope $S(\omega)$, the electric field of the pulses in the time domain can be written as a Fourier series:

$$
E(t)=\sum_{N=-\infty}^{\infty} S\left(\omega_{N}\right) e^{-j\left(N \omega_{\mathrm{r}}+\omega_{\mathrm{CE}}\right) t} .
$$

One cavity roundtrip period $T=2 \pi / \omega_{\mathrm{r}}$ later, the electric field equals:

$$
\begin{aligned}
E(t+T) & =\sum_{N=-\infty}^{\infty} S\left(\omega_{N}\right) e^{-j\left(N \omega_{\mathrm{r}}+\omega_{\mathrm{CE}}\right)(t+T)} \\
& =E(t) e^{-j\left(2 N \pi+2 \pi \omega_{\mathrm{CE}} / \omega_{\mathrm{r}}\right)} \\
& =E(t) e^{-j \Delta \varphi}, \text { with: } \\
\Delta \varphi & :=2 \pi \frac{\omega_{\mathrm{CE}}}{\omega_{\mathrm{r}}} .
\end{aligned}
$$

In other words, the electric field of each pulse produced by the laser is a copy of the electric field of the previous pulse, shifted by a phase $\Delta \varphi$. Due to the often used timedomain representation of the electric field as a continuously oscillating carrier wave times an envelope function, $\Delta \varphi$ is also called the "carrier-envelope offset phase slippage", which also explains the name of $\omega_{\mathrm{CE}}$. Figure 2.3 visualizes the evolution of the carrier-envelope 


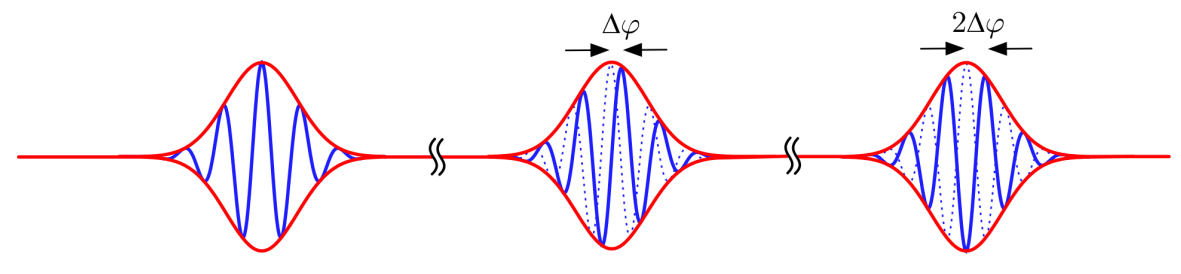

Figure 2.3: Pulse-to-pulse evolution of the carrier-envelope offset for $\Delta \varphi=\pi / 2$ (as in [40]).

offset for the slippage phase $\Delta \varphi=\pi / 2$. Stabilizing the pulse-to-pulse phase of a modelocked laser is crucial for localizing the comb modes in the frequency domain as well as for time-domain applications which demand the reproducibility of the electric field rather than just that of the intensity profile. Carrier-envelope phase measurement and stabilization has been made possible in the last decade, see e.g. [38 43, 48 50].

The basic property enabling the enhancement of the pulses generated by a mode-locked oscillator in a passive cavity is the pulse-to-pulse reproducibility of the electric field, which is equivalent to the comb spectrum. Depending on the purpose of the enhancement, the pulses generated by the mode-locked oscillator can be further processed without affecting this basic property. Such processes include: pulse picking: reduction of the repetition rate; this generates new spectral components, in such a way that the comb spacing equals the reduced repetition rate; sideband generation: generation of additional frequency components through fast modulation, particularly useful for the Pound-Drever-Hall locking scheme (see Section 2.1.3); pulse chirping: manipulation of the spectral phase, in particular, allows for a variation of the pulse peak power while keeping the spectral components and the energy constant; linear power amplification: scaling up the pulse energy while keeping the other parameters constant; comb-preserving harmonic generation: creates a frequency comb with the original comb spacing at a multiple of the central frequency.

\subsubsection{Passive Enhancement in an External Cavity}

Similar to a mode-locked laser, the steady-state condition of an enhancement cavity requires a pulse which is reproduced after each roundtrip. However, in contrast to a mode-locked oscillator, the latter does not incorporate an intracavity mechanism for dispersion compensation. Rather, the optical resonances of cavity, which are continuously excited by the seeding frequency comb modes, are not equidistant in the frequency domain due to roundtrip dispersion. This distinction from the seeding frequency comb affects the interference of the seeding with the circulating field, representing an enhancement limitation. Moreover, the fact that the circulating field has a different spectral amplitude and phase distribution than the seeding field requires increased attention when controlling the interference of the two. In the following, we first derive an analytical model for the steady state of a passive cavity excited by a frequency comb. Then, we use this model to discuss the trade-off between enhancement finesse and bandwidth. 
input coupler: reflectivity $R(\omega)$, transmission $T(\omega)$

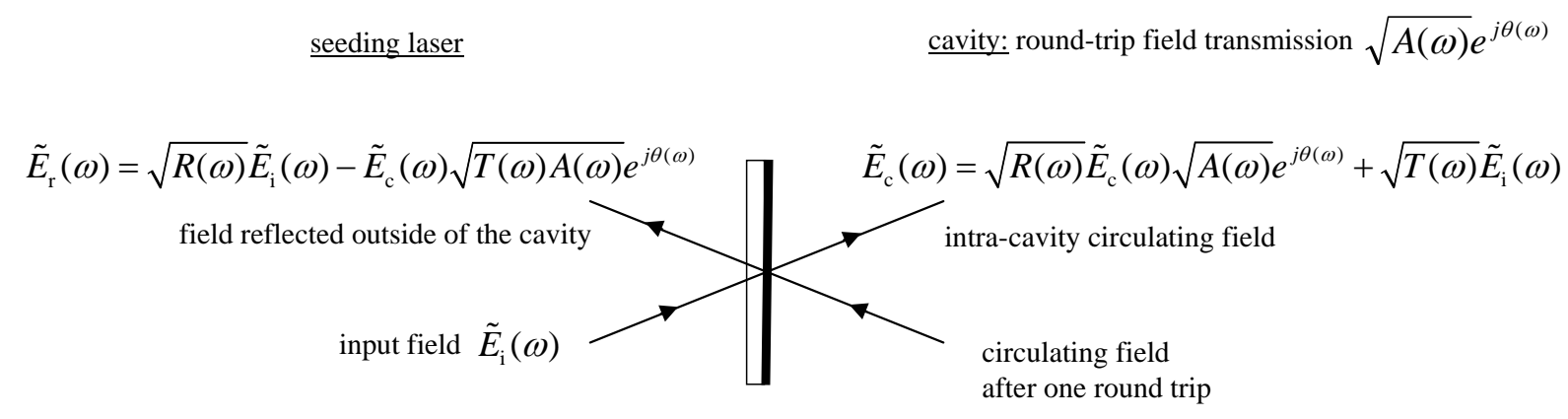

Figure 2.4: Electric fields at the input coupler of an enhancement cavity in the steady state.

\section{The Steady State}

Figure 2.4 shows the complex electric fields at the input coupler (IC) of an enhancement cavity in the steady state. Usually, enhancement cavities for nonlinear conversion are implemented as ring cavities. This is to avoid a double pass in opposite directions through the nonlinear material and to enable a straightforward spatial separation of the field seeding the cavity and the field reflected by the IC1. The following derivation, however, holds for both linear and ring cavities. Moreover, we assume perfect transverse mode matching at the IC, i.e. that the seeding laser beam is matched to the excited cavity transverse mode. For each frequency component $\omega$, the single-roundtrip power attenuation and accumulated phase are denoted by $A(\omega)$ and $\theta(\omega)$, respectively. Thus, one cavity roundtrip of the circulating electric field component $\widetilde{E}_{\mathrm{c}}(\omega)$ can be completely described by its multiplication by $\sqrt{A(\omega)} \exp [j \theta(\omega)]$. Let $R(\omega)$ denote the IC power reflectivity, so that a reflection on the cavity side at the IC implies the multiplication of the impinging electric field $\widetilde{E_{\mathrm{c}}}(\omega)$ by $\sqrt{R(\omega)}$. Let $T(\omega)$ denote the IC power transmission. Resonant enhancement requires the constructive interference of the intracavity field reflected by the IC, i.e., $\sqrt{R(\omega)} \widetilde{E}_{\mathrm{c}}(\omega) \sqrt{A(\omega)} \exp [j \theta(\omega)]$ with the portion of the input field transmitted through the IC, i.e., $\sqrt{T(\omega)} \widetilde{E}_{\mathrm{i}}(\omega)$. In the steady state the sum of these two interfering fields equals $\widetilde{E_{\mathrm{c}}}(\omega)$ :

$$
\begin{aligned}
\widetilde{E}_{\mathrm{c}}(\omega) & =\sqrt{R(\omega)} \widetilde{E}_{\mathrm{c}}(\omega) \sqrt{A(\omega)} \exp [j \theta(\omega)]+\sqrt{T(\omega)} \widetilde{E}_{\mathrm{i}}(\omega) \\
\Leftrightarrow \widetilde{H}(\omega):=\frac{\sqrt{T(\omega)}}{\widetilde{E}_{\mathrm{c}}(\omega)} & =\frac{\sqrt{E_{\mathrm{i}}(\omega)}}{1-\sqrt{R(\omega) A(\omega)} \exp [j \theta(\omega)]} .
\end{aligned}
$$

\footnotetext{
${ }^{1}$ This comes in handy especially when using seeding systems sensitive to optical feedback.
} 
For linear, i.e. intensity-independent behavior of the cavity, the Airy function $\widetilde{H}(\omega)$ defined in eq. 2.12 represents the transfer function of the cavity. However, the ratio $\widetilde{H}(\omega)$ evaluated over the spectrum of the input field also bears significance if intracavity nonlinear processes are involved. In particular, $\widetilde{H}(\omega)$ can be measured with high accuracy (see Section 4.2.2 providing information on the nonlinear process itself.

In a similar fashion, the superposition $\widetilde{E}_{\mathrm{r}}(\omega)$ of the input field reflected by the IC outside of the cavity and the intracavity field transmitted through the IC can be calculated:

$$
\widetilde{E}_{\mathrm{r}}(\omega)=\sqrt{R(\omega)} \widetilde{E}_{\mathrm{i}}(\omega)-\widetilde{E}_{\mathrm{c}}(\omega) \sqrt{T(\omega) A(\omega)} \exp [j \theta(\omega)] .
$$

Note that the minus sign between the two terms stems from a phase shift of opposed sign compared to the one between the two terms on the right-hand side of eq. (2.11). This follows from the Fresnel equations and can also be explained by energy conservation. By plugging eq. (2.12) in the above equation, we obtain:

$$
\frac{\widetilde{E}_{\mathrm{r}}(\omega)}{\widetilde{E}_{\mathrm{i}}(\omega)}=\frac{\sqrt{R(\omega)}-[R(\omega)+T(\omega)] \sqrt{A(\omega)} \exp [j \theta(\omega)]}{1-\sqrt{R(\omega) A(\omega)} \exp [j \theta(\omega)]} .
$$

The entire seeding energy is coupled to the cavity if the numerator of the right-hand side of Eq. 2.14) equals 0. This impedance matching condition is fulfilled for a frequency $\omega$, if on the one hand the roundtrip phase at this frequency is a multiple of $2 \pi$ and on the other hand the reflectivity and transmission of the IC are matched to the roundtrip amplitude losses. For a lossless IC, i.e. if $R(\omega)+T(\omega)=1$ holds, the second impedance matching condition becomes $R(\omega)=A(\omega)$.

Two further notions, historically stemming from optical interferometry, are important in the context of passive cavities. The free spectral range FSR of a resonator (or interferometer) denotes the resonance spacing in the frequency domain. For a dispersive cavity, the FSR is $\omega$-dependent and $F S R(\omega)=\pi c_{0} /[2 n(\omega) L]$ holds, where $c_{0}, n(\omega)$ and $2 L$ denote the speed of light in vacuum, the frequency-dependent refractive index for the intracavity propagation and the cavity geometrical length, respectively. The cavity finesse $\mathcal{F}$ is a measure of the resolving power of the resonator used as a transmission filter (in the sense of an etalon or interferometer). It is defined as the ratio of the FSR to the FWHM bandwidth of a cavity resonance $\Delta \omega_{\text {cav }}$, see e.g. Section 11.5 in [2]:

$$
\mathcal{F}(\omega):=\frac{F S R(\omega)}{\Delta \omega_{\text {cav }}} .
$$

With the field propagation coefficient $r_{\text {tot }}(\omega):=\sqrt{R(\omega) A(\omega)}$ summing up the total losses of the frequency $\omega$ upon a roundtrip propagation in the passive resonator, the following equation holds for $\Delta \omega_{\text {cav }}$ (cf. eq.(52) in [2], Section 11.5):

$$
\begin{aligned}
\Delta \omega_{\text {cav }} & =\frac{4 c}{2 L} \arcsin \left(\frac{1-r_{\mathrm{tot}}(\omega)}{2 \sqrt{r_{\mathrm{tot}}(\omega)}}\right) \\
& \approx\left(\frac{1-r_{\mathrm{tot}}(\omega)}{\pi \sqrt{r_{\mathrm{tot}}(\omega)}}\right) \cdot \operatorname{FSR}(\omega) .
\end{aligned}
$$


It follows for the finesse:

$$
\begin{aligned}
\mathcal{F}(\omega) & \approx \frac{\pi \sqrt{r_{\mathrm{tot}}(\omega)}}{1-r_{\mathrm{tot}}(\omega)} \\
& \approx \frac{2 \pi}{1-r_{\mathrm{tot}}^{2}(\omega)}=\frac{2 \pi}{1-R(\omega) A(\omega)}
\end{aligned}
$$

Thus, the finesse is given by the resonator losses and is independent of the resonator length.

\section{Summary of Useful Formulas}

In the following, we sum up the main equations for two important special cases:

- Assumptions: no dispersion, i.e. $\omega$-dependence is discarded, lossless IC, i.e. $R+T=1$ holds, and the cavity is on resonance. Then eqns. (2.12) and (2.14) imply for the power enhancement $P$ and the reflected portion of the input light Re $f$ :

$$
\begin{aligned}
P:=|\widetilde{H}(\omega)|^{2} & =\left|\frac{\widetilde{E}_{\mathrm{c}}(\omega)}{\widetilde{E}_{\mathrm{i}}(\omega)}\right|^{2}=\frac{T}{(1-\sqrt{R A})^{2}}, \\
\operatorname{Refl} l & :=\left|\frac{\widetilde{E}_{\mathrm{r}}(\omega)}{\widetilde{E}_{\mathrm{i}}(\omega)}\right|^{2}=\left(\frac{\sqrt{R}-\sqrt{A}}{1-\sqrt{R A}}\right)^{2} .
\end{aligned}
$$

- Assumptions: like above but also impedance matching is given, i.e. $R=A$ holds:

$$
\begin{aligned}
P & =\frac{1}{T}, \\
\text { Refl } & =0, \\
\mathcal{F} & =\pi P .
\end{aligned}
$$

\section{Enhancement Trade-off: Finesse versus Bandwidth}

The roundtrip phase $\theta(\omega)$ describes the phase changes of a wave of frequency $\omega$ upon propagating in the cavity (usually in a transverse eigen-mode of the cavity) and interacting with its optics. Let us assume that a certain frequency $\omega_{c}$ is resonant in the cavity, i.e. the roundtrip phase accumulated by a wave with this frequency is a multiple of $2 \pi$. Then, $\theta(\omega)$ can be expanded in a Taylor series about $\omega_{\mathrm{c}}$ :

$$
\theta(\omega)=\theta_{0}+G D\left(\omega-\omega_{\mathrm{c}}\right)+G D D\left(\omega-\omega_{\mathrm{c}}\right)^{2}+\ldots,
$$

where $\theta_{0}, G D$ and $G D D$ denote a constant phase, the group delay and the group delay dispersion 2 , respectively. The constant phase term $\theta_{0}$ has only a physical significance if a

\footnotetext{
${ }^{2}$ Note that the GDD and the GVD are linked by the relationship $\theta(\omega)=k(\omega) d$ which implies $G D D(\omega)=$ $G V D(\omega) d$, where $d$ is a distance. While the GVD measures the dispersion upon propagation through a material in general, the $G D D$ is used to characterize the dispersion of optical elements with a fixed geometry.
} 


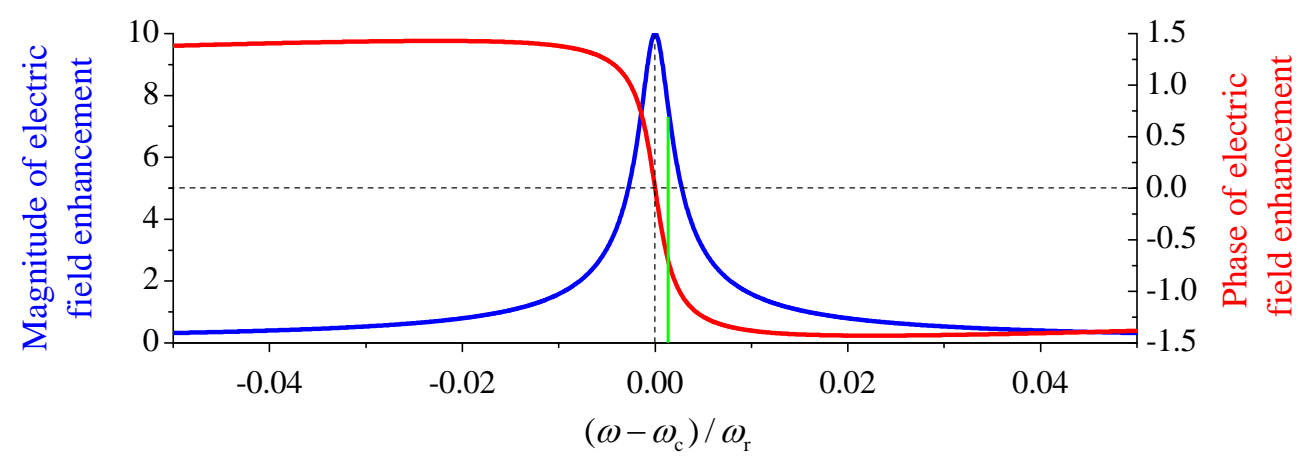

Figure 2.5: Resonance of an impedance-matched cavity with a power enhancement of 100, centered at $\omega_{\mathrm{c}}$. Green line: continuous wave with a slightly different frequency than $\omega_{\mathrm{c}}$, would experience a lower enhancement and a phase shift in the cavity.

phase difference is considered, e.g. between two transverse modes of different orders (cf. Section 2.1.4). The group delay is determined by the repetition frequency of the pulse circulating in the cavity according to $G D=2 \pi / \omega_{\mathrm{r}}$ and can usually be easily varied by tuning the geometrical cavity length.

Figure 2.5 shows the magnitude and phase of $\widetilde{H}(\omega)$ for an impedance-matched (dispersionfree) cavity with a power enhancement of 100 , which is resonant at $\omega_{\mathrm{c}}$, over a frequency range extending $5 \%$ of $\omega_{\mathrm{r}}$ to the left and to the right of $\omega_{\mathrm{c}}$. If a continuous wave with a slightly different frequency than $\omega_{\mathrm{c}}$ is exciting this cavity resonance (see e.g. green line in Fig. 2.5), the wave oscillating in the resonator will not only experience a smaller power enhancement than that of a wave with $\omega_{c}$, but also a constant phase shift with respect to the exciting wave.

Equation (2.12) implies that roundtrip dispersion, i.e. $G D D$ and higher-order terms in eq. 2.25 and also frequency-dependencies of $\sqrt{A(\omega)}$ affect both the amplitude and the phase of $\widetilde{H}(\omega)$. In particular, this means that the resonances of a real cavity are not equidistant in contrast to the seeding frequency comb. Thus, some of the comb modes will necessarily be offset from the centers (magnitude peaks) of the corresponding cavity resonances, having a twofold effect on the circulating field. Firstly, the spectrum will not be evenly enhanced, meaning a spectral filtering of the seeding frequencies. Secondly, the phase of the circulating field will differ from the one of the input field. The dispersion imposed on the circulating field amounts to approximately the power enhancement factor times the roundtrip dispersion of the cavity (a derivation can be found in Section 2.4 of the paper included [34, see Chapter 6). A higher cavity finesse increases both effects. Thus, when dimensioning an enhancement cavity, a trade-off between finesse and bandwidth is necessary. Fig 2.6 illustrates this trade-off. The same frequency comb (green lines) is enhanced in a cavity with high finesse and in a cavity with low finesse. The high-finesse cavity enhances the central mode to a high degree but filters out the other modes strongly 


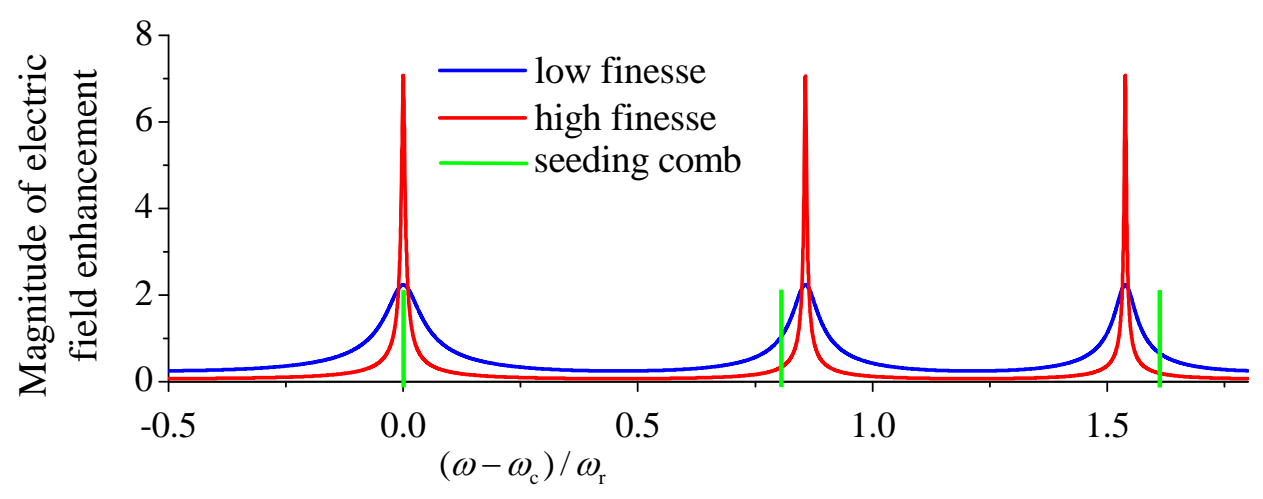

Figure 2.6: Qualitative illustration of the enhancement finesse vs. bandwidth trade-off. Green: seeding comb frequencies, red: high-finesse cavity with dispersion, blue: low-finesse cavity with same dispersion. Spectral filtering and phase distortions are less pronounced for the low-finesse cavity. However, the power enhancement at the central mode is also smaller than in the high-finesse case.

and also strongly affects the phase of the circulating field. The spectral filtering and the applied dispersion are much smaller in the case of the low-finesse cavity. However, the power enhancement in the latter is also smaller. This trade-off is clearly noticeable in the enhancement cavity systems demonstrated so far, cf. Appendix 7.1.

\subsubsection{Interferometric Stabilization}

The strict condition of interferometric overlap of the input field with the intracavity circulating field at all times, usually implies the need for active locking of the seeding comb to the cavity resonances or viceversa. The example in Appendix 7.2 shows that even small length deviations on a picometer scale, common to a normal lab environment, can alter the enhancement significantly. In this section we first address the control variables in the context of comb-cavity locking and review the most common locking schemes. Then, we derive quantitative conditions under which the lock of the seeding comb and the enhancement cavity can be realized with a single feedback loop. This is particularly relevant for the design of an experimental setup.

In our discussion we assume (i) that all fluctuations are slow enough and (ii) that the cavity response $\widetilde{H}(\omega)$ is linear, so that the steady states described in the previous two sections can be assumed. These assumptions usually apply for most mechanical and electronic distortions and they allow the discussion to be carried out in the frequency domain by using the comb and the cavity models given by eqns. (2.7) and (2.12), respectively. We stress that processes on shorter time scales, such as nonlinear intracavity interactions, might introduce additional fluctuations, which are not accounted for by this discussion. Section 3.2.2 addresses this in more detail. 


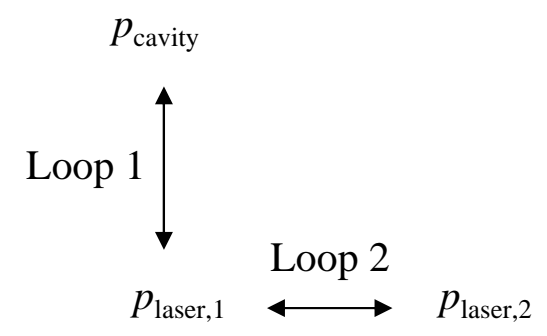

Figure 2.7: Feedback-loop connection of the parameters $p_{\text {laser, } 1,} p_{\text {laser }, 2}$ and $p_{\text {cavity }}$, enabling optimum overlap of the seeding comb with the cavity resonances. Active locking is indicated by the arrows.

\section{The Optimum Overlap}

The frequency comb described by eq. (2.7) can be parameterized by using two independent parameters. The two parameters need not necessarily be $\omega_{\mathrm{r}}$ and $\omega_{\mathrm{CE}}$ and for the sake of generality we will call them $p_{\text {laser,1 }}$ and $p_{\text {laser,2, }}$. In contrast, under the assumptions made above, the comb-lik $\Theta^{3}$ structure of the enhancement cavity response in the frequency domain, given by eq. (2.12), is completely described by the propagation length along the cavity optical path for a given set of (dispersive) cavity optics. In other words, the cavity transfer function can be parameterized by a single parameter (once the cavity optics are given), which we will refer to as $p_{\text {cavity }}$.

In analogy to the trade-off between finesse and enhancement bandwidth discussed in the previous section, the spectral filtering property of the cavity owed to dispersion implies that different combinations of $p_{\text {laser, } 1,} p_{\text {laser, } 2}$ and $p_{\text {cavity }}$ might be optimal for different enhancement purposes. For instance, obtaining the shortest possible circulating pulse or obtaining the highest enhancement in a certain spectral region might call for a different overlap between the frequency comb modes and the cavity resonances in the same setup (cf. e.g. [51]). In general, there exists an optimum overlap of the seeding comb modes with the cavity resonances, which can be described by a well defined combination of $p_{\text {laser }, 1}$, $p_{\text {laser }, 2}$ and $p_{\text {cavity }}$.

The train of thought followed so far has two immediate consequences. On the one hand, the single cavity parameter does not in general suffice to reach optimum overlap, since the seeding comb requires two parameters for a full description. Thus, at least one degree of freedom of the overlap needs to be controlled by means of the seeding comb. On the other hand, if one of the comb parameters, say $p_{\text {laser, }, 1}$, and the cavity parameter $p_{\text {cavity }}$ are locked, the other comb parameter, i.e. $p_{\text {laser, } 2}$, is unambiguously determined by $p_{\text {laser, } 1}$ according to a constraint set by the optimum overlap (e.g. shortest intracavity pulse or highest intracavity power). In particular, this means that $p_{\text {laser, } 2}$ can be locked to $p_{\text {laser }, 1}$, without a direct feedback from the cavity, which is illustrated in Fig. 2.7.

\footnotetext{
${ }^{3}$ By the formulation "comb-like" we wish to emphasize that the cavity resonances are not equidistant.
} 


\section{Choice of the Control Parameters - Practical Considerations}

The main fluctuations causing deviations from the optimum overlap and occurring in every real experimental setup are of mechanical and electrical nature. In general, mechanical vibrations affect all parameters, while electrical fluctuations affect mostly the laser (see e.g. [52, 53] ), since the cavity is passive. Due to the effect of mechanical vibrations on the lengths of both the oscillator cavity and the enhancement cavity, it is common to stabilize the length of one of these cavities to match the length of the other one. If an enhanced frequency comb is desired for which the absolute frequencies are highly precise (e.g. for spectroscopy), then it is customary to lock the enhancement cavity to the seeding laser (which is locked to an external reference). However, if the precise knowledge of the absolute value of the enhanced modes is not mandatory, as is the case with most timedomain applications, the passive cavity can be used as a reference and one of the oscillator parameters can be locked to the cavity parameter. In practice this implementation is usually less demanding due to the lower power in the oscillator cavity (see also Section 4.1). In conclusion, we can thus assume without loss of generality that the cavity resonances are fixed, i.e. $p_{\text {cavity }}$ is constant, and the two comb parameters are used to compensate deviations from the optimum overlap, which can be divided into two independent locking tasks (cf. Fig,2.7):

- Loop 1: $p_{\text {laser }, 1}$ controls the frequency of a comb mode (e.g. $\omega_{\mathrm{c}}$ ), so that this mode is locked to (usually the peak of) a cavity resonance, which is determined by $p_{\text {cavity }}$.

- Loop 2: $p_{\text {laser,2 }}$ is set by using only feedback from $p_{\text {laser, } 1 \text {, with the boundary condition }}$ that optimum overlap is reached.

The complex dynamics of mode locking (see e.g. [47,52]) makes it very difficult to vary the two comb parameters $\omega_{\mathrm{r}}$ and $\omega_{\mathrm{CE}}$ completely independently. In general, the available control mechanisms (like piezoelectric actuators, acousto- and electro-optical modulators, dispersion management, optical pump power variation, etc.) act on both $\omega_{\mathrm{r}}$ and $\omega_{\mathrm{CE}}$. Therefore, in practice, the parameters $p_{\text {laser, } 1}$ and $p_{\text {laser,2 }}$ are two linear combinations of $\omega_{\mathrm{r}}$ and $\omega_{\mathrm{CE}}$, which need to satisfy two necessary conditions: (i) they need to be linearly independent and (ii) they need to be tunable over a region large enough around the optimum overlap so that all occurring fluctuations can be compensated for. Under these two conditions, the comb needs to be brought in the vicinity of the optimum overlap in the parameter space defined by $p_{\text {laser, } 1}$ and $p_{\text {laser,2 }}$ by coarse adjustment and, subsequently, active stabilization will ensure an optimum overlap. In the following paragraph, we review the most common schemes for the two locking tasks, i.e. $p_{\text {laser }, 1}$ to $p_{\text {cavity }}$ and $p_{\text {laser }, 2}$ to $p_{\text {laser, } 1 \text {. }}$ 


\section{Common Locking Schemes}

An active feedback loop consists of three main components: an error signal, indicating the deviation of the actuating variable from the desired optimum, locking electronics setting the control mechanism according to this error signal, and the control mechanism itself. For the lock of a comb line to a Fabry-Perot cavity with an Airy-function response as given by eq. (2.12) and plotted in Fig. 2.5, the control mechanism can be a piezo-actuated mirror, the optical pump power of the oscillator, an acousto- or electro-optical modulator etc.. The locking electronics are usually a standard, commercially available phase-locked loop. Rather than providing a deep insight into the mode of operation of these standard electronic components, we address in the following, the generation of the error signal, which represents the interface of the optical setup, and the task of interferometric stabilization, which is an electronic one.

The necessary property every error signal needs to fulfill, is bipolarity, i.e. the signal should indicate unambiguously the direction (and magnitude) of the drift which needs to be compensated for. For example, the intensity of the optical signal transmitted through a cavity mirror, as a function of the mismatch between the driving frequency and the cavity resonance, is not suited as an error signal for locking the peak of the resonance, since the Airy function is an even function (see Fig. 2.5). However, this signal is suited to lock the driving frequency to a sub-maximum level on one side of the Airy fringe, e.g. the position indicated by the green comb line in Fig. 2.5. This scheme is usually referred to as side-offringe locking and was first employed to stabilize (CW) lasers to a reference cavity [54] rather than to enhance a field with the purpose of increasing the available power.

Schemes able to lock a driving frequency to the peak of a cavity resonance require a bipolar error signal that equals 0 at the peak of the resonance and changes its sign when the mismatch passes through this zero. Such signals are usually obtained from observing the interference of a portion of light in phase with the driving frequency ("reference") and a portion of intracavity light that contains the phase information of the cavity ("sample").

The Hänsch-Couillaud or polarization locking scheme [55] works for polarization discriminating cavities. The linearly polarized light seeding the cavity needs to contain nonzero components in two orthogonal polarization directions, e.g. parallel and perpendicular to the intracavity polarization discrimination direction. For each polarization component, the overall field reflected by the cavity is given by eq. (2.14) and represents the superposition of a portion of the seeding light which serves as the "reference" and a portion of transmitted light, which carries the phase information of the cavity and serves as the "sample" part of the interferometer. The "sample" portion is linearly polarized, but, due to the polarization discrimination, its decomposition along the two polarization directions has a different ratio of coefficients than the reference part of the beam. When the cavity is on resonance, the phase difference between the two linearly polarized parts is 0 so that their superposition is linearly polarized. However, when the cavity is off-resonance, the sample part has a frequency-dependent phase shift with respect to the reference part, manifesting itself as an elliptical polarization of the reflected beam. The direction and the magnitude of the ellipticity can readily be detected with an analyzer consisting of a quarter-wave plate, a 
polarizing beam splitter and a difference photodiode, providing the error signal. The cavity in the proof-of-principle paper [55] contains a Brewster plate which provides strong polarization discrimination. However, recently we have shown that the nonorthogonal incidence of the beam on the cavity mirrors provides a polarization discrimination large enough for a stably working Hänsch-Couillaud lock 33,34]. The results presented in this thesis have been obtained using the Hänsch-Couillaud locking scheme.

Several locking schemes, which we summarize under the name of transverse mode mismatch locking, use the fact that an optically stable cavity has a set of well-defined transverse eigen-modes. If this cavity is excited externally and a mismatch between the exciting beam and the excited transverse mode is introduced, a spatial interference of the part of the input beam which is not matched to the cavity mode (reference) with the beam resonant in the cavity (sample) can be produced. A photodetector placed at a proper position generates a bipolar error signal. The mismatch can be e.g. given by a non-modematched input beam [56, 57] or by a tilted input beam [58]. While these methods are easy to implement and low-cost, they are inherently prone to misalignment and to changes of the input beam and the excited cavity transverse mode.

One of the most robust and widely used stabilization schemes today is the PoundDrever-Hall locking scheme, see e.g. 59 61 and references therein. The method was initially developed by Pound [62] for microwave frequency stabilization of and later adapted for optical oscillators [59]. By modulating the input light with an RF source (local oscillator), sidebands of the frequency to be locked are produced. If the distance of the sidebands from the resonant frequency in the frequency domain is large enough so that these sidebands are not resonant, the sidebands can be used as the reference. The signal reflected by the input coupler of the resonant cavity carries the desired phase information and can be used as the sample signal in a phase-sensitive heterodyne detection scheme which demodulates the reflection signal against the RF source [59].

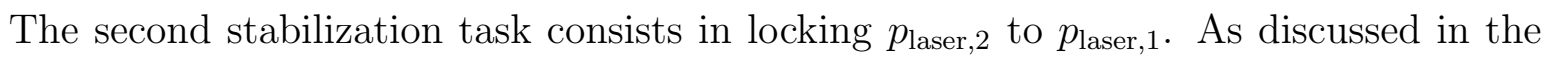
beginning of this subsection, this stabilization concerns the laser only. While the repetition frequency of the laser is easy to access as an RF signal from a photodiode, the detection of the CE phase slippage $\Delta \varphi$ is somewhat more intricate. The most common and straightforward scheme for the detection of $\omega_{\mathrm{CE}}$ is based on an $f$-to- $2 f$ interferometer, see e.g. [40] and references therein. Here, an octave-spanning frequency comb is employed to generate a beat signal between the mode with the number $N$ and the one with the number $2 N$. Such a broad spectrum can be generated e.g. by self-phase modulation in a nonlinear crystal. The two frequencies $\omega_{N}=N \omega_{\mathrm{r}}+\omega_{\mathrm{CE}}$ and $\omega_{2 N}=2 N \omega_{\mathrm{r}}+\omega_{\mathrm{CE}}$ generate a beat note with an RF frequency corresponding to their frequency difference, i.e. $\omega_{2 N}-\omega_{N}=\omega_{\mathrm{CE}}$. Controlling $\omega_{\mathrm{CE}}$ can be done in manifold ways. A slow control can be obtained by varying the length of the oscillator beam path through a dispersive material, which influences the ratio of the group to the phase velocity, see e.g. 63. Recently, a related method was demonstrated in which a composite plate is used to vary this ratio without affecting the repetition rate [64]. Faster controls can be achieved by modulating the power of the pumping beam, see e.g. [52, 53, 65 or by shifting the pumping beam with respect to the cavity mode 66]. 
a) Optimum Overlap

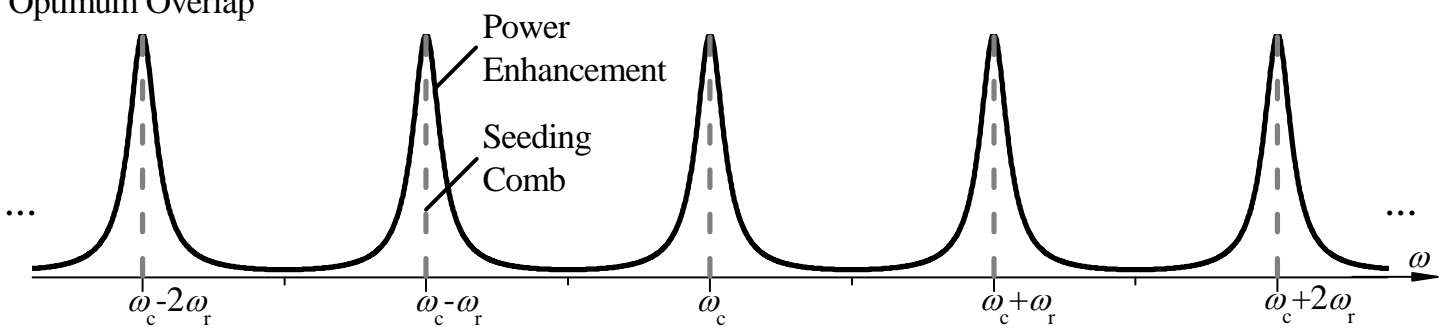

b) Shift of $\omega_{\mathrm{CE}}$ by $\Delta \omega_{\mathrm{CE}}$

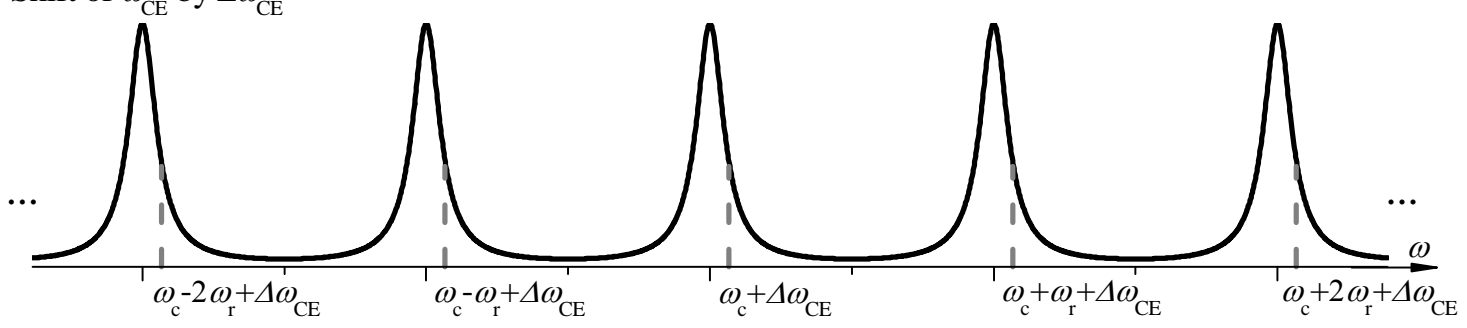

c) Compensation of $\Delta \omega_{\mathrm{CE}}$ through $\Delta \omega_{\mathrm{r}}$ at $\omega_{\mathrm{c}}$

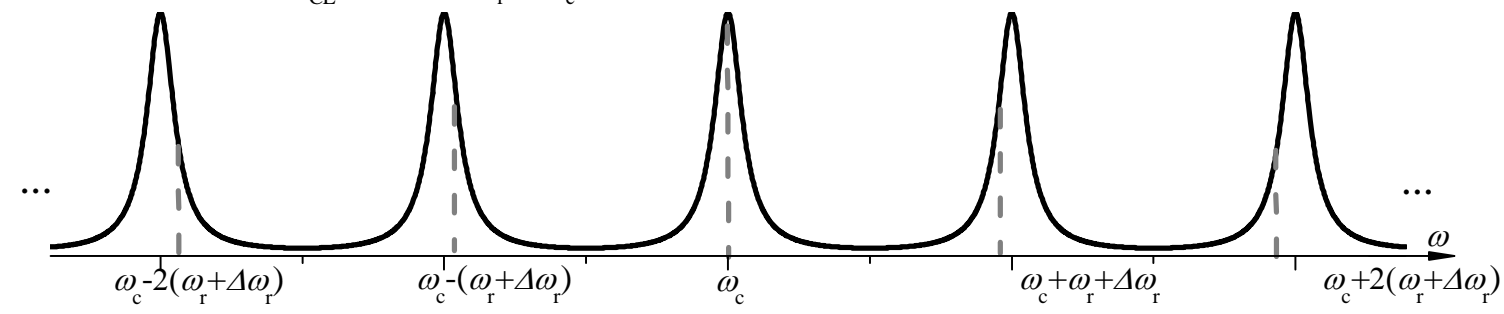

Figure 2.8: Illustration of the effect of a shift $\Delta \omega_{\mathrm{CE}}$ of the carrier-envelope offset frequency $\omega_{\mathrm{CE}}$ on the enhanced frequency comb, if the laser-cavity lock is implemented by controlling the repetition frequency $\omega_{\mathrm{r}}$ so that the central optical frequency $\omega_{\mathrm{c}}$ is locked to the peak of a cavity resonance.

\section{Locking a Single Degree of Freedom}

The necessity to actively control a second degree of freedom (i.e. the lock of $p_{\text {laser }, 2}$ to $\left.p_{\text {laser,1 }}\right)$ mainly depends on the jitter, on the optical bandwidth, on the cavity finesse and on the required stability of the enhanced frequency comb. For many practical applications, fluctuations of two orthogonal degrees of freedom, e.g. of $\omega_{\mathrm{r}}$ and $\omega_{\mathrm{CE}}$ around the state of optimum overlap have a very similar effect on the enhancement, making a second active loop unnecessary. This will be shown quantitatively in the following calculation, in close analogy to Section 2.1 of our paper 67.

For our derivation, we assume that the central optical frequency $\omega_{\mathrm{c}}$ is being locked to the peak of a corresponding cavity resonance by actively controlling solely the repetition frequency $\omega_{\mathrm{r}}$ of the comb, and we calculate the effect of a variation $\Delta \omega_{\mathrm{CE}}$ of the other comb 
parameter $\omega_{\mathrm{CE}}$ on the enhancement. In the context of the laser-cavity lock the following description of $\theta(\omega)$ is convenient:

$$
\theta(\omega)=\tau \omega+\psi(\omega)=2 \pi \frac{\omega}{\omega_{\mathrm{r}}}+\psi(\omega)
$$

where $\tau=2 \pi / \omega_{\mathrm{r}}$ is the round trip group delay and $\psi(\omega)$ is a term describing the residual intra-cavity dispersion. For simplicity, here we assume that $\psi(\omega)=0$ and that an initial $\omega_{\mathrm{CE}}$ is given, which leads to an optimum overlap of the seeding comb modes with the cavity resonances, see Fig. 2.8. a. Let $N_{\mathrm{c}}$ denote the mode number of $\omega_{\mathrm{c}}$, so that

$$
\omega_{\mathrm{c}}=N_{\mathrm{c}} \omega_{\mathrm{r}}+\omega_{\mathrm{CE}}
$$

holds. A variation of the comb parameter $\omega_{\mathrm{r}}$, meaning a change of the comb mode spacing, can be entirely compensated for by the active control. In contrast, a variation $\Delta \omega_{\mathrm{CE}}$ of the carrier-envelope offset frequency shifts the entire frequency comb, as depicted in Fig. 2.8. b. The active control compensates the drift of $\omega_{\mathrm{c}}$ by introducing a variation $\Delta \omega_{\mathrm{r}}$ to $\omega_{\mathrm{r}}$ :

$$
\omega_{\mathrm{c}}=N_{\mathrm{c}}\left(\omega_{\mathrm{r}}-\Delta \omega_{\mathrm{r}}\right)+\omega_{\mathrm{CE}}+\Delta \omega_{\mathrm{CE}}
$$

The last two equations imply:

$$
\Delta \omega_{\mathrm{r}}=\Delta \omega_{\mathrm{CE}} / N_{\mathrm{c}}
$$

While the mode with the number $N_{\mathrm{c}}$ retains its initial frequency, the mode with the number $N_{\mathrm{c}}+m$ will experience a frequency shift equal to $m \Delta \omega_{\mathrm{r}}$. This situation is depicted in Fig. 2.8.c.

In Fig. 2.9, the effect of a variation $\Delta \omega_{\mathrm{CE}}$, corrected by a variation of $\omega_{\mathrm{r}}$ as described above, on the enhanced pulse is plotted for several enhancement factors and several optical bandwidths. As a result of this correction the circulating power decreases and the intracavity spectrum is narrowed relative to the case of optimum overlap of the seeding comb with the cavity resonances. The relative changes are plotted as functions of $\Delta \omega_{\mathrm{CE}}$ and of $\Delta \omega_{\mathrm{CE}} /(2 \pi) \Delta \omega_{\text {cav }}$, where $\Delta \omega_{\text {cav }}$ is the full-width-half-maximum of the cavity resonance. The optical bandwidths used in the calculated examples represent typical values used in femtosecond enhancement cavity experiments. The majority of the experiments presented in this thesis are performed with parameters close to the 7-nm case shown in Fig. 2.9. c. Throughout these experiments, the seeding comb could be locked to the cavity by using a single feedback loop, cf. Section 4.1. However, a high-finesse enhancement of pulses with broader bandwidths might require a second feedback loop, cf. Section 5.1.2.

\subsubsection{Transverse Modes, Cavity Scan and Quasi-Imaging}

The transverse modes of a resonator (and also of free space or a waveguide) are selfconsistent transverse electromagnetic field distributions. A textbook treatment of optical resonator transverse modes is given in Sections 1.6 and 14.3 of Siegman's book Lasers [2]. The most relevant theoretical aspects for the work presented in this thesis are presented in Section 2 of the paper included [37], see Chapter 6. In this section we emphasize two further aspects. 
a)

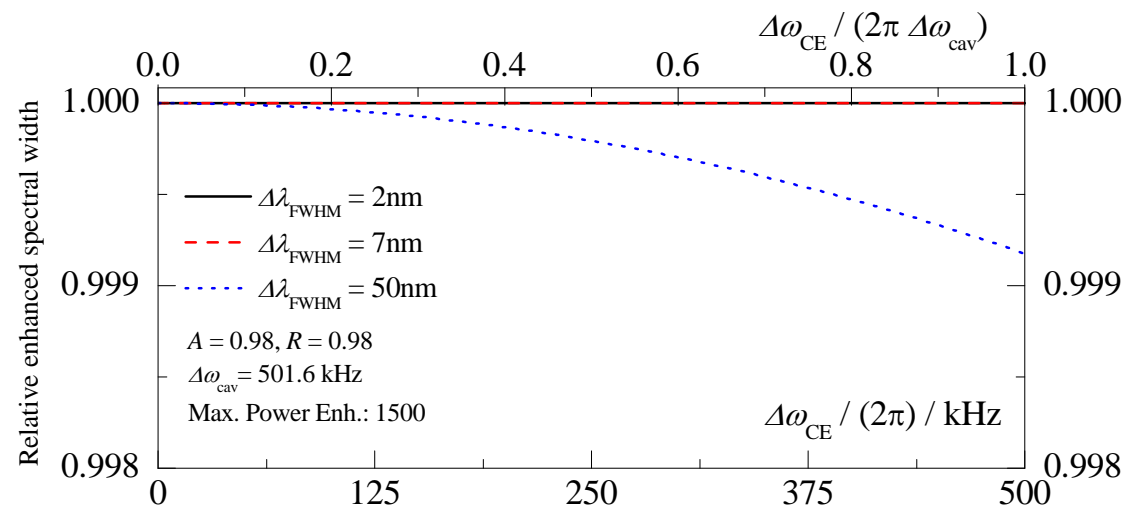

b)
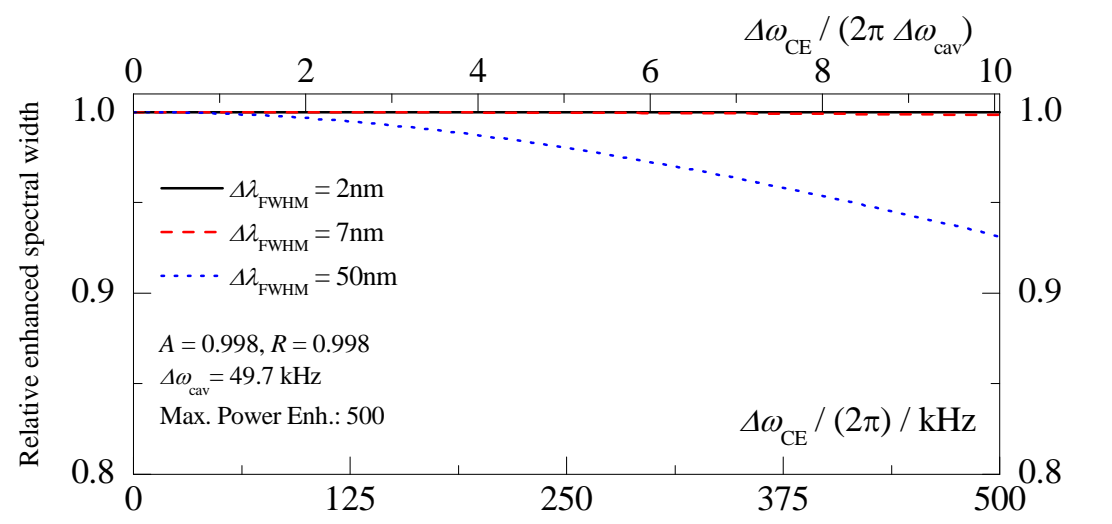

c)

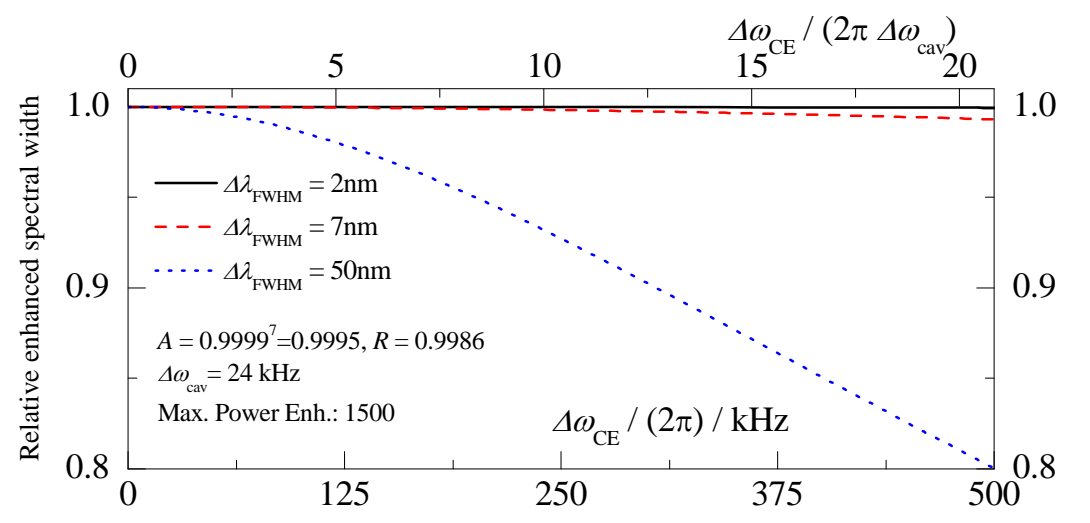

Figure 2.9: Calculated reduction of the enhanced spectral width (FWHM) after a carrierenvelope offset frequency drift $\Delta \omega_{\mathrm{CE}}$ has been compensated for by a shift of the repetition frequency $\omega_{\mathrm{r}}$, locked at the central optical frequency $\omega_{\mathrm{c}}$. The reduction is related to the case of optimum overlap between the frequency comb modes and the cavity resonances. A Gaussian input spectrum is assumed. In a good approximation, the total power enhancement is proportional to the spectral narrowing and the pulse lengthening is inversely proportional to the spectral narrowing. (a) and (b): impedance matched cases with power enhancements at $\omega_{\mathrm{c}}$ of 50 and 500, respectively. (c): power enhancement of 1500 at $\omega_{\mathrm{c}}$, not impedance matched, corresponding to our experimental setup. 


\section{Cavity Scan}

The scan of the oscillator length generating the seeding pulses around the value of the enhancement cavity length is explained in Section 2.3 of the paper included [37] (see Chapter 6) and in Figures 3 and 5.b therein, in the context of adjusting the relative phase between higher-order transverse modes. This is particularly useful to enable the simultaneous resonance of a subset of cavity eigen-modes, which can then be coupled by means of obstacles in the beam path to tailor new low-loss transverse modes. This technique, which we call quasi-imaging, is addressed in the next paragraph. Besides enabling quasiimaging adjustment the scan pattern is also a powerful diagnostic tool, as discussed in the following 4 . Section 4.2 .2 includes examples for all points listed here.

- Optimum overlap of the axial comb modes of the seeding pulses with cavity resonances. The scan pattern can be used to adjust the two laser parameters and the cavity parameter manually and coarsely so as to be in the vicinity of the optimum overlap so that subsequent active stabilization ensures a steady state in the optimum overlap, as discussed in the previous section. For sufficiently small cavity dispersion, this is achieved when the main resonance in the scan pattern reaches a maximum and the peaks of the two resonances, one free spectral range to the left and to the right of the main resonance, have equal heights.

- Transverse mode matching. The scan pattern also provides information on the amount of light coupled to transverse modes other than the desired one. For instance, in Section 3 of [37] the peaks corresponding to higher-order transverse modes are used to calculate the overlap of the input beam with the excited modes. If the target alignment is a perfect transverse mode matching then these peaks must vanish.

- Error signal adjustment. As discussed in the previous section, the bipolar error signal for locking should indicate unambiguously the direction and magnitude of the drift which needs to be compensated. Important parameters of the error signal, like amplitude and balancing can be adjusted while scanning over a cavity resonance.

- Finesse and nonlinearities. For a given seeding frequency comb, the ratio of the main peak to the ones located a free spectral range to the left and to the right provides a measure for the spectral filtering of the cavity (as discussed in the previous section), which increases with a higher finesse and with dispersion, both of which might also be intensity-dependent. This diagnostic is particularly useful in power scaling experiments, where the (average or peak) power of the seeding comb is increased while all other parameters are kept constant.

\footnotetext{
${ }^{4}$ The frequency-domain scan of a Fabry-Perot resonator is not only a helpful diagnostic for the enhancement in this resonator, but a measurement tool in its own right: a cavity with variable length can be used as a passive tunable filter or as a scanning interferometer, see e.g. Section 11.5 in 2], scanning a CW laser through a cavity resonance can be employed for ringdown measurements 68] etc.
} 
- Stability range detuning and roundtrip phase difference between the sagittal and tangential directions. As explained in [37], the scan pattern contains information about the detuning from the center of the stability range (see also next paragraph). Moreover, a difference of the Gouy parameters for the $x$ and the $y$ directions in the cavity leads to different on-axis phase 5 of the higher-order modes with the same sum of $x$ and $y$ indices. This results in a multitude of peaks in the scan pattern corresponding to a group of resonances with identical index sum. Conversely, the roundtrip phase difference between the $x$ and the $y$ directions can be determined from this scan pattern (if the finesse is large enough so that these peaks are well resolved). For example, Fig. 5.b. in Section 3 of [37] shows a case in which the Gouy parameters are $\psi_{x}=0.78 \cdot 2 \pi$ and $\psi_{y}=0.76 \cdot 2 \pi$. In a stable resonator, in which the beam lies in a single plane, this difference is caused by the difference of the effective radius of curvature of the (de)focusing mirrors thus, indicating the angles of incidence on these mirrors. In an out-of-plane stable resonator this diagnostic can e.g. be used to adjust the angles of incidence on the curved mirrors so that the Gouy parameters in both directions are equal. In this case all peaks corresponding to modes with the same sum of $x$ and $y$ indices coincide. In particular, this could be used to adjust quasi-imaging in both directions, see Section 5.2.2.

\section{Mode Degeneracy and Quasi-Imaging}

A phenomenon which can be very useful if controlled properly, and harmful if unwanted, is transverse mode degeneracy. Several transverse modes of different orders can be simultaneously resonant. Intracavity phase front distortions can then lead to a coupling of these modes [69], influencing the excited mode in the cavity.

On the one hand, if fundamental-mode operation is desired this phenomenon leads to a degradation of the beam quality, as discussed in [69]. Such phase front distortions can for instance be induced by thermal or nonlinear effects in the resonator optics. We have observed this in experiments involving an intracavity Brewster plate for XUV output coupling, cf. Section 4.4.1. It has also been reported that the gas target can lead to such phase front distortions [70]. The consequence of this unwanted effect is an increase of the roundtrip losses for the distorted mode, possibly a decrease of the overlap with the exciting mode and thus, overall a decrease of the enhancement and of the HHG efficiency. The scan pattern can be used to adjust the relative phases of the transverse modes to avoid resonant coupling among them 6 .

On the other hand, the phenomenon of transverse mode degeneracy, together with the proper choice of obstacles in the beam path and of the seeding transverse mode, enables the controlled combination of eigen-modes of a cavity resulting in a tailored mode with desired properties. An example thereof is the quasi-imaging (QI) concept presented in the paper included [37], see Chapter 6 (see also Section 5.2). The initial motivation of the de-

\footnotetext{
${ }^{5}$ The accumulation of different on-axis phases manifests itself in different optical cavity lengths.

${ }^{6}$ However, the average power during the scan is different from the steady-state regime. Therefore, distortions due to thermal effects might not be visible in the scan pattern.
} 
velopment of QI was to obtain a field distribution which simultaneously exhibits an on-axis maximum for HHG in a gas target close to a cavity focus and, after a certain propagation distance, an on-axis low-intensity region for coupling out the intracavity generated XUV radiation through an opening in a cavity mirror in this region. However, this application can be regarded as a special case of the more general concept of exciting a combination of degenerate transverse modes in a cavity, among which the coupling can be tuned with high precision by means of the position in the stability range. This enables the confinement of different processes to spatially separated regions within the cavity and, at the same time, a precise control of the energy coupling between these regions and therefore between the respective processes. This concept might prove to be useful for tasks other than providing a direct on-axis access to a high-finesse cavity ${ }^{7}$

\subsection{High-Harmonic Generation (HHG) in a Gas}

For longer than the past two decades the process of HHG has been extensively studied, both experimentally and theoretically. Owing to its immediate relation to the generation of XUV radiation, such studies have been playing a central role at our institute. In particular, in the context of enhancement cavities, theoretical descriptions of HHG have recently found their way into several $\mathrm{PhD}$ theses, e.g. $22,71,73]$. Rather than reproducing in detail the different models describing HHG, this section briefly outlines the semiclassical HHG model known as the three step model, and subsequently addresses phase matching considerations, aimed at providing an intuitive introduction to HHG and at enabling the interpretation of the experimental results presented here as well as of the challenges faced towards further power scaling. At the end of the section, we sum up scaling laws for HHG.

\subsubsection{The Three-Step Model and Phase-Matching}

\section{The Three-Step Model of Single-Atom HHG}

The specific structure of high-harmonic spectra generated by multi-cycle laser pulses consists of odd harmonics, whose envelope exhibits: (i) an exponential fall-off (perturbative region) for the lower-order harmonics, (ii) a plateau region of nearly equal power per harmonic, succeeded by (iii) a cutoff in the short-wavelength part of the spectrum. Experimentally, this structure has first been observed in the late eighties [74, 75]. The three step model, developed by Corkum and coworkers, see [76] and references therein, provides a semiclassical, intuitive approach to $\mathrm{HHG}$, explaining many of its properties. In 1994, Lewenstein and coworkers justified this model with quantum mechanical considerations [77]. In the following, we outline the three step model, explaining the response of a single atom to a high-intensity field. The model is illustrated in Fig. 2.10.

\footnotetext{
${ }^{7}$ For instance, it might enable the implementation of the seeding oscillator resonator (with its active medium and mode-locking mechanism) and the passive enhancement resonator in the same cavity (here, cavity designates a hollow space rather than a resonator). This could simplify the experimental setup considerably and in particular, spare the necessity of active synchronization.
} 


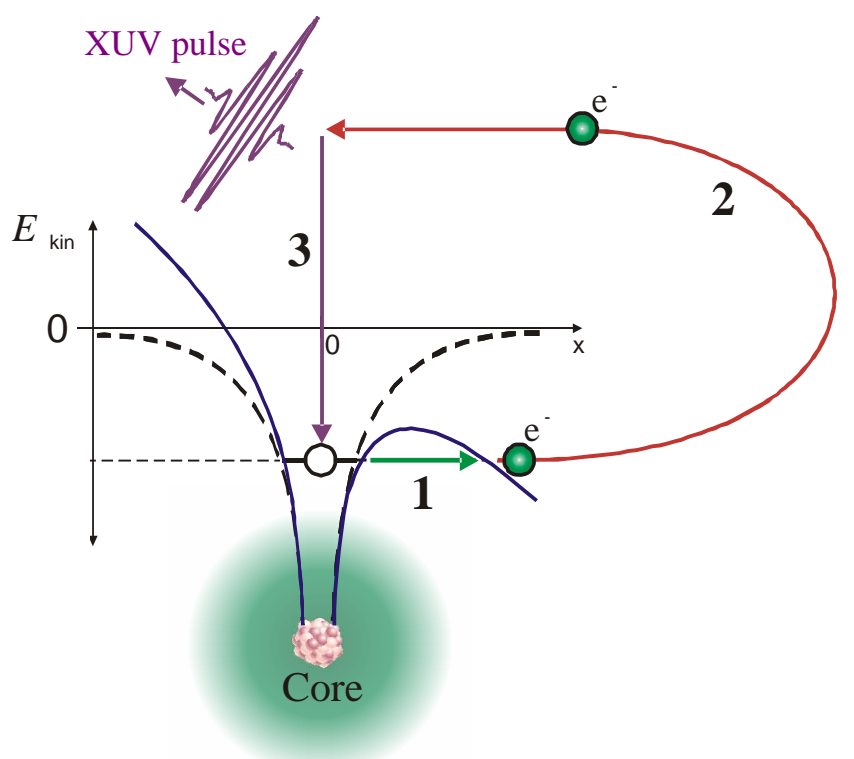

Figure 2.10: Three step model: 1 - tunnel ionization, 2 - acceleration through the laser field, 3 - recombination to the ground state and emission of an XUV photon. This image is reproduced from [78 by courtesy of Matthias Kling.

\begin{tabular}{l|c|c|c|c|c} 
Gas & $\mathrm{Xe}$ & $\mathrm{Kr}$ & $\mathrm{Ar}$ & $\mathrm{Ne}$ & $\mathrm{He}$ \\
\hline$U_{\mathrm{i}} / \mathrm{eV}$ & 12.13 & 13.99 & 15.76 & 21.6 & 24.6
\end{tabular}

Table 2.1: Ionization potentials (from Section 12.2.1 in [5]).

In the first step, the highly intense driving electric field bends the Coulomb potential barrier of the atom so that an electron tunnels from the atomic ground state. A necessary condition for this model to hold is that the so-called Keldysh parameter

$$
\gamma=\sqrt{\frac{U_{\mathrm{i}}}{2 U_{\mathrm{p}}}}
$$

is smaller than one [71]. Here, $U_{\mathrm{i}}$ is the ionization potential of the atom, see Tab. 2.1, and $U_{\mathrm{p}}$ is the ponderomotive energy, i.e. the average kinetic energy of an electron in a harmonic field, given by:

$$
\begin{aligned}
U_{\mathrm{p}} & =\frac{e^{2} E_{0}^{2}}{4 \omega^{2} m_{e}} \\
& =\frac{e^{2}}{4 \omega^{2} m_{e}} \cdot \frac{2 I}{c \epsilon_{0}},
\end{aligned}
$$

where $e, m_{e}, \omega, \epsilon_{0}, E_{0}$ and $I$ are the electron charge and mass, the optical frequency, the vacuum permittivity, the electric field amplitude and the laser beam intensity, respectively. 
Plugging in the natural constants and using the common measurement units $\mathrm{W} / \mathrm{cm}^{2}$ and $\mu \mathrm{m}$ for $I$ and $\lambda$, respectively, yields:

$$
U_{\mathrm{p}} \approx 9.33 \cdot 10^{-14} I \lambda^{2} .
$$

For $\gamma<1$ the tunneling times of the electron through the bent Coulomb barrier are short compared to the optical cycle, and the tunnel ionization model implies the formation of a sequence of wave packets, one close to each electric field maximum of the driving multi-cycle laser pulse.

The second step concerns the acceleration of the electron in the laser electric field. It uses classical mechanics to describe well-defined trajectories for the electrons. The trajectories along which the electron wave packets return to the parent ion lead to recombination, which constitutes the third step. Upon recombination, a photon is emitted with an energy equal to the sum of the kinetic energy acquired during acceleration and the ionization potential, i.e. $\hbar \omega=E_{\text {kin }}+U_{\mathrm{i}}$. The trajectory and thus, the energy of the emitted photon, depends on the time, i.e. the phase of the driving field, at which the ionization takes place. The model implies that the highest kinetic energy is reached if the electron tunnels at $\omega t \approx 0.3+k \pi \mathrm{rad}$ for any integer $k$. In this case, the absolute maximum of the kinetic energy amounts to $3.17 U_{\mathrm{p}}$, which defines the cutoff frequency via:

$$
\hbar \omega_{\text {cutoff }}=3.17 U_{\mathrm{p}}+U_{\mathrm{i}}
$$

The typical odd-harmonic spectrum originates from the interference of the spectra of the radiation emitted by the different optical cycles of the driving pulse. The cycle-to-cycle repetition of the wave packets passing the parent ions implies that any light that is emitted will be at an odd harmonic of the laser frequency. In the case of single-cycle driving pulses, this interference vanishes and the harmonic spectrum exhibits a rather continuous shape.

Another important result is that only close-to-linear polarization leads the tunneled electron back to the atom and thus to recombination. This is also the key to all isolated as-pulse generation schemes involving polarization gating, see Section 5.3 .

\section{Multi-Atom HHG - Phase Matching}

The response of a macroscopic target, such as a gas jet or cell, to a driving field depends on the spatial phase relation between the radiation contributions of each atom involved in the HHG process, i.e. on phase matching. On the one hand, the coherent addition of these contributions is essential for efficient XUV generation. On the other hand, multiple factors, like the intensity-dependent phases of the excited dipoles, the macroscopic properties of the emitting medium as well as the driving beam space and time properties influence phase matching, making it a complex problem. A thorough treatment of HHG phase matching exceeds the scope of this thesis. Here, the discussion is restricted to an overview of the main factors determining phase matching.

Firstly, the atomic phase of the participating dipoles is intensity-dependent and thus, a function of space and time. Section 11.2 of [5] gives an introduction hereof. An important 
result is that two geometries are found for which good phase matching is achieved. One of them corresponds to collinear phase matching and results in a Gaussian-like emitted harmonic beam. The other one corresponds to noncollinear phase matching and yields an annular beam.

Secondly, for a Gaussian beam with the confocal parameter

$$
b=\frac{2 \pi w_{0}^{2}}{\lambda},
$$

where $w_{0}$ is the $1 / e^{2}$-intensity decay focus radius, the geometrical phase shift with respect to a plane wave (Gouy phase shift), depends on both the axial and radial coordinates, $z$ and $r$, respectively. For a Gaussian beam with a focus at $z=0$ and a wavefront curvature $R(z)$, the geometrical phase is given by the expression:

$$
\phi_{\text {geom }}(r, z)=-\arctan \left(\frac{2 z}{b}\right)+\frac{\pi r^{2}}{\lambda R(z)} .
$$

The intensity-dependence of the atomic dipole moment together with the geometrical phase lead to a substantial dependence of the coherence properties of the generated harmonics on the position of the nonlinear medium with respect to the focus and on its geometry (see e.g. 79 for details). Assuming a homogenous transverse distribution of the nonlinear medium, the geometry of the interaction region is mainly described by its length $l_{\text {med }}$. If $l_{\text {med }}<b$ holds, the system is referred to as exhibiting weak (or loose) focusing. For $l_{\text {med }}>b$ the focusing is said to be tight. As a rule, optimum phase matching is reached employing loose focusing conditions 80 82. The high quality of the transverse mode of an enhancement cavity favors phase matching.

Thirdly, the atomic density plays an essential role in phase matching since dispersion introduces a phase mismatch $\Delta k=k_{q}-q k_{1}$ between the phase $k_{q}$ of the harmonic field of order $q$ and the phase $k_{1}$ of the fundamental (polarization) field. In a dispersive medium, the phase lag between the nonlinear polarization introduced by the fundamental laser, oscillating with the frequency $\omega$, at the frequency $q \omega$ and the free Gaussian beam propagating in the medium is [83]:

$$
\Delta \phi(z)=(1-q) \arctan \left(\frac{2 z}{b}\right)+\Delta k z .
$$

The values of $\Delta k$ for a fundamental wavelength close to the one employed in our experiment are given in Tab. 1 in [84] for $q$ between 3 and 33. Another important contribution to the phase mismatch is caused by the free electrons of the ionized gas, see also Tab. 1 in 84. The influence of several gas target parameters on the harmonic yield is discussed in Section 12.2.2 in [5]. In general, an optimum interplay of the mechanisms contributing to phase matching can be found empirically, by adjusting the geometry, the position and the pressure of the gas nozzle. 


\subsubsection{Scaling Laws}

For the design (dimensioning) of any HHG device, the knowledge of the scaling laws of this highly nonlinear process is imperative. However, as opposed to the cases of many other nonlinear processes, in the case of HHG in a gas, a simple and general scaling model, consisting of closed expressions of all relevant experimental parameters, which predict the spectrally resolved intensity of the generated high harmonics, still lacks 8 . This is partly due to the complexity of the process and the multitude of involved parameters, but also due to the absence of HHG experiments enabling the validation of theoretical models over large parameter variation ranges. In particular, the strong intensity-dependence of the process makes the comparison between different experiments difficult. In this section we sum up the most relevant HHG scaling laws, which have been confirmed by all experiments so far.

For a better overview we first list the formulas linking the pulse energy $E_{\text {pulse }}$, the average power $P_{\mathrm{av}}$, the repetition frequency (rate) $f_{\text {rep }}$, the pulse peak power $P_{\text {peak }}$, the pulse duration $\tau_{\text {pulse }}$ and the peak intensity $I$ of a pulse with a Gaussian distribution in time and space:

$$
\begin{aligned}
E_{\text {pulse }} & =\frac{P_{\mathrm{av}}}{f_{\text {rep }}}, \\
P_{\text {peak }} & =0.94 \frac{E_{\text {pulse }}}{\tau_{\text {pulse }}}, \\
I & =2 \frac{P_{\text {peak }}}{A_{\text {focus }}}=2 \frac{P_{\text {av }}}{f_{\text {rep }} \tau_{\text {pulse }} \pi w_{0}^{2}} .
\end{aligned}
$$

Eqns. 2.32) and (2.34) imply for the cutoff frequency:

$$
\hbar \omega_{\text {cutoff }}=2.96 \cdot 10^{-13} I \lambda^{2}+U_{\mathrm{i}} .
$$

Thus, higher harmonics are generated using a longer driving wavelength. In particular, among the most common mode-locked lasers, this constitutes an advantage of Yb-based systems (centered around $1030 \mathrm{~nm}$ ) over Ti:Sa systems (centered around $800 \mathrm{~nm}$ ). Scaling the cutoff by means of the intensity can be done by (see eq. (2.40): (i) increasing the pulse peak power, meaning increasing $P_{\text {av }}$ and/or decreasing $\tau_{\text {pulse }}$ and/or decreasing $f_{\text {rep }}$. The challenges related to these scaling measures from the point of view of enhancement cavities are discussed in Section 3.2.1. (ii) by tightening the focusing. However, this usually alters phase matching (see previous section). Moreover, increasing the intensity is limited by ionization. The probability for the latter increases with the intensity, leading to an eventual breakdown of HHG. For example, for Xe the optimum intensity for HHG lies in the range $\sim 10^{13}-10^{14} \mathrm{~W} / \mathrm{cm}^{2}$.

Furthermore, the cutoff can be pushed towards higher frequencies by choosing lighter atoms with a larger ionization potential (see Tab. 2.1) since $\omega_{\text {cutoff }}$ depends on $U_{\mathrm{i}}$ via eq. (2.34).

\footnotetext{
${ }^{8}$ First closed-form relations for the conversion efficiency under the assumption of a square-shaped pulse are provided in 85. However, the focusing conditions and intensity-dependent phase matching are left out of this model.
} 
Concerning the conversion efficiency, a compendium of several HHG experiments is provided in Section 3.1.2. of [86]. A theoretical study [87] for driving lasers from the nearvisible $(800 \mathrm{~nm})$ to the mid-infrared $(2 \mu \mathrm{m})$, has recently predicted a dramatic conversion efficiency $(C E)$ improvement for HHG driven by shorter wavelengths:

$$
C E \propto \lambda^{-6} \ldots \lambda^{-5} .
$$

This scaling law has been experimentally verified in the range 800 - $1850 \mathrm{~nm}$ for Xe $(C E \propto$ $\left.\lambda^{-6.3 \pm 1.1}\right)$ and $\operatorname{Kr}\left(C E \propto \lambda^{-6.5 \pm 1.1}\right)[88$.

In weak focusing operation (i.e. for $l_{\text {med }}<b$ ), at constant driving intensity, the intensity of plateau harmonics $I_{\text {plateau }}$ is observed to scale according to the following law (see Section 12.2 .1 in $[5,89])$ :

$$
I_{\text {plateau }} \propto b^{3} .
$$

The transverse mode characteristics of the generated harmonics depend strongly on the fundamental beam shape, the intensity and the phase matching conditions. In practice, spatial coherence measurements can be performed (cf. e.g. Section 12.1.2 in [5]). For HHG with a focused Gaussian beam the following approximation of the divergence of the harmonics holds (cf. Section 4.2.1 in [71]):

$$
\begin{aligned}
& \frac{\theta_{\text {perturb }}^{q}}{\theta_{\mathrm{f}}} \propto \frac{1}{\sqrt{q}}, \\
& \frac{\theta_{\text {plateau }}^{q}}{\theta_{\mathrm{f}}} \propto \frac{1}{q},
\end{aligned}
$$

where $q, \theta_{\mathrm{f}}, \theta_{\text {perturb }}^{q}$ and $\theta_{\text {plateau }}^{q}$ denote the harmonic order, the divergence of the fundamental beam with $\theta_{\mathrm{f}}=\lambda /\left(\pi w_{0}\right)$, the divergence of the $q^{\text {th }}$ harmonic in the perturbative regime (exponential decay, low harmonics) and the divergence of the $q^{\text {th }}$ plateau harmonic, respectively.

Like in the case of the $\lambda^{-(6 . .5)}$ and the $b^{3}$-laws in eqns. 2.42) and 2.43), respectively, formulating a scaling law for the harmonic yield with respect to the pulse duration $\tau_{\text {pulse }}$ requires a constant intensity. However, unlike in the aforementioned two cases, where the variables $\lambda$ and $b$ have a well-defined influence on the HHG process, the variation of $\tau_{\text {pulse }}$ and the subsequent adjustment of the intensity to a constant level, is not a well-defined operation. For instance, $\tau_{\text {pulse }}$ can be varied by modifying the amplitude spectrum of the pulse and/or the spectral phase. The latter influences the distances between the zeros of the electric field rather than their number, which determines the number of HHG events. Moreover, the intensity envelope of the pulse, which can exhibit a complex shape, influences the ionization of the gas during the interaction with the pulse, implying saturation and dispersion processes. Thus, the terms pulse duration and intensity are too coarse to fully describe the multitude of possibilities related to these quantities. Despite this shortcoming several special cases can be found in the literature. In [5,89] it is stated that the harmonic yield is proportional to $\tau_{\text {pulse }}$. This however, follows from the assumption hat the number 
of XUV photons generated at a given intensity is proportional to the pulse duration, which only holds in the absence of ionization ${ }^{9}$. In [90] and [91 the pulse duration is reduced from $750 \mathrm{fs}$ to $120 \mathrm{fs}$ and from $200 \mathrm{fs}$ to $25 \mathrm{fs}$, respectively, by removing an imposed chirp. In both cases the intensity is kept constant by decreasing the pulse energy along with its temporal compression. While the first publication reports no dependence of the harmonic distribution on the pulse duration, the second one states that shorter pulses lead to an increased conversion efficiency. Moreover, fewer oscillations per pulse, which in the transferlimited case means shorter pulses, imply broader spectra of the individual harmonics.

\footnotetext{
${ }^{9}$ For shorter pulses, the saturation intensity is higher, allowing to drive HHG at higher intensities. In

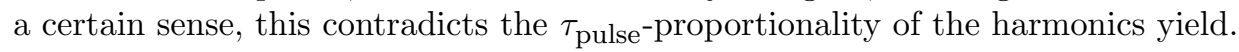




\section{Chapter 3}

\section{Objectives of the Experiment and Technological Challenges}

The objectives of the experiments presented in this thesis are formulated in Section 3.1 . The technological challenges on the way to achieving these objectives are reviewed in Section 3.2. This section also gives an overview of the solutions developed during the course of this work and of the remaining challenges.

The grand goals of the research in the field of high-repetition-rate, passively-enhanced HHG are pushing the cut-off of the HHG process towards higher energies and scaling up the power and the bandwidth of each harmonic. From the point of view of the enhancement cavity technology, these goals pose analogous challenges as with any other intracavity high-intensity interaction process. Therefore, the objectives formulated in the following can be easily translated to other applications, and the results presented in this thesis have significant implications beyond high-repetition-rate HHG.

\section{$3.1 \quad$ Objectives}

A fundamental constraint of the scaling of the enhancement cavity technique towards the goals mentioned above is the ionization of the nonlinear medium, see Section 2.2.2. Since the intensity $I$ of the fundamental radiation is given by the ratio of the peak power $P_{\text {peak }}$ to the focus size $A_{\text {focus }}$, efficient scaling can only be performed if both are increased. While increasing $A_{\text {focus }}$ is a relatively straight-forward cavity-design task, scaling up the peak power is technologically challenging and will be discussed in the following. Moreover, increasing the focus size (i.e. loose focusing) is also desirable due to the $b^{3}$-scaling law for the intensity of the plateau harmonics.

Increasing $P_{\text {peak }}$ can be achieved by increasing the pulse energy $E_{\text {pulse }}$ and/or decreasing the pulse duration $\tau_{\text {pulse }}$, which is equivalent to increasing the enhancement bandwidth. For a fixed repetition rate (which is e.g. given by the envisaged application), scaling up $E_{\text {pulse }}$ means increasing the average power $P_{\mathrm{av}}$. The enhancement-cavity-related challenges concerning the increase of $P_{\mathrm{av}}$ and the decrease of $\tau_{\text {pulse }}$ are discussed in Section 3.2.1. 
In conclusion, a viable power scaling strategy consists of the following objectives:

- increasing the average power $P_{\mathrm{av}}$,

- decreasing the pulse duration $\tau_{\text {pulse }}$,

- optimizing the HHG process by increasing the focus size $A_{\text {focus }}$ according to the scaling of the first two.

- coupling out the intracavity generated harmonics efficiently.

Currently, from the point of view of the seeding lasers, Yb-based systems are the most appropriate sources for pursuing the first two objectives, see also Section 5.1.2. Furthermore, Yb-based lasers emit radiation at a longer wavelength than Ti:Sa-systems, which are the closest alternative for seeding high-power femtosecond enhancement cavities. Due to the $\lambda^{2}$-scaling law of the cutoff frequency of the high harmonics, this constitutes another advantage of the Yb-based systems. Conversely, the $\lambda^{-(6 \ldots 5)}$-scaling law for the conversion efficiency implies a roughly 3 to 4 times lower conversion for Yb-based systems. However, this shortcoming can usually be readily compensated by means of an average power increase. It should also be mentioned that the highly desirable generation of isolated aspulses offers the most promising prospects in conjunction with short pulses (see Section 5.3), which is in accordance with the second objective. Moreover, extending the operation range of cavity-based HHG towards higher average powers and shorter pulses will lead to a better understanding of the HHG process itself by enabling the variation of isolated parameters over larger ranges.

\subsection{Challenges}

\subsubsection{Power and Bandwidth Scaling of an Empty Cavity}

\section{Average and Peak Power Scaling for a Constant Finesse}

The optics for a given cavity design impose circulating average and peak power limitations. To push the damage threshold of the cavity and enable power scaling these limitations need to be investigated and overcome. One of the major results of this thesis is the investigation of the power scaling of a standard-design bow-tie cavity consisting of commercially available, state-of-the art dielectric mirrors, see Section 4.3. In particular, it was found that power scaling is primarily limited by intensity effects in the cavity mirrors. On the one hand, this insight led to the development of next-generation enhancement cavity designs, see Section 5.1.1. The design goal of these novel cavities is to increase the spot size on all the mirrors, thus decreasing the intensities on the mirrors. On the other hand, while the novel designs are expected to allow for average and peak power scaling beyond the levels presented in this thesis, they present new challenges. Firstly, average-power-related effects, 
such as absorption, scattering and thermal lensing are expected to play an increasingly important role. Secondly, increased spot sizes in general request an increased mechanical stability. These aspects are discussed in Section 5.1.1. Besides improved cavity designs, mirror development promises to contribute to pushing the current power scaling limits.

\section{Broad Bandwidth versus High Finesse}

Enhancing seeding pulses with shorter durations, i.e. increased bandwidths, represents manifold challenges in a high-finesse cavity. Firstly, non-zero roundtrip group-delay dispersion leads to spectral filtering and chirps magnification in the steady state, see Section 2.1.2. Secondly, the broader the bandwidth and the higher the cavity finesse, the larger is the difference between the influence of drifts of the two orthogonal frequency comb degrees of freedom on the comb-cavity overlap, cf. Section 2.1.3. Thus, the requirements of the comb-cavity synchronization become more strict.

These effects need to be taken into account when designing the reflectivity and phase properties of the cavity mirrors in the frame of the technological limitations. In particular, trading in power enhancement (or cavity finesse) for a broader circulating bandwidth might be useful. Owing to the relatively narrow optical bandwidth of the pulses used in the experiments presented in this thesis, the effects mentioned above only played a role when intracavity nonlinearities were pronounced. However, for further bandwidth scaling, special attention needs to be paid to these effects, cf. Section 5.1.1.

In the course of this thesis a diagnostic method was developed, which allows for the fast and accurate determination of the cavity roundtrip dispersion, including even intracavity nonlinear effects. This method (described in Section 4.2.2) is expected to be instrumental in the design process of mirrors for future bandwidth scaling.

\subsubsection{Inclusion of a Gas Target}

The mode of operation of femtosecond enhancement cavities relies on the superposition of the intracavity circulating pulse with every pulse of the seeding train at each bounce off the input coupler. Any distortion of the intracavity pulse affects this superposition and therefore, the performance of the cavity. From this point of view, nonlinear cavity dynamics $70,92,94$ and in particular, the interaction of the fundamental radiation with the gas target, require special attention.

Very recently, two publications have tackled the still open question of the effect of the intracavity plasma on the cavity performance 70,94$]$. The majority of the experimental findings reported there have been qualitatively observed in our system as well (see e.g. Section 3.3 in the paper enclosed [34] in Chapter 6). In our group a thorough analysis of these effects remains a subject for future study. In the following we review the most important laser-gas interaction mechanisms influencing the performance of the cavity and constituting future challenges related to power scaling. 


\section{Effect on Longitudinal Modes}

The interaction of the high-intensity fundamental radiation with the gas affects both the amplitude and the phase of the circulating field. The amplitude effect is due to the energy which the laser loses when producing photoelectrons and increases if the interaction volume is increased, see e.g. [95].

There are two processes with characteristic timescales on the order of the pulse repetition period [70]: (i) the plasma decay and (ii) the travel time of gas atoms in the interaction region. If the pulse repetition period is shorter than these two times, the number of ionized atoms and of electrons in the interaction region will be large and dispersion will affect the circulating beam, cf. Section 2.2.1. Moreover, ionization is accompanied by self-phase modulation (SPM) and a spectral blue-shift, resulting in additional dispersion and affecting the spectral overlap at the input coupler.

To avoid ionization and enable efficient HHG from a large interaction volume, finding the optimum intensity will be necessary and will presumably play a more important role than in experiments with smaller interaction volumes. Moreover, the prevention of cumulative effects becomes more important as the interaction volume increases, due to the longer travel time of gas atoms through the high-intensity region.

\section{Effect on Transverse Mode}

The plasma also acts as a negative lens, defocusing the beam and influencing the excited cavity transverse mode [70]. If several transverse modes are degenerate (or close to degeneracy), this phase front distortion might lead to a coupling among them, as explained in Section 2.1.4. To avoid this, a proper position in the stability range, where coupling among modes is strongly suppressed and/or spatial filters might be useful. Moreover, this effect might be detrimental to the quasi-imaging technique (see Section 2.1.4), where the coupling among modes of different transverse orders is crucia!

\section{Effect on Lock Stability}

As discussed above, the nonlinear cavity response due to the gas target has a dynamic nature. This might lead to locking instabilities and/or irreproducibility, a fact which has been observed experimentally in our system and in 70,94 . One of the main reasons for this is that the error signal for the lock of the cavity parameter and one of the laser parameters (see Section 2.1.3) is usually generated from an isolated (and narrow) part of the enhanced spectrum. In principle, the narrower this optical bandwidth, the more accurate is the confinement of the error signal generation to a spectral region, which ideally extends over a single cavity resonance. However, an extremely narrow bandwidth also means a poor SNR. Therefore, in practice this bandwidth extends over many cavity free spectral ranges, which, in the case of a nonlinear response, fluctuate according to the dynamic nature of the optical dispersion.

\footnotetext{
${ }^{1}$ However, in principle it could be pre-compensated for by proper transverse mode matching.
} 
Usually, the the error signal for locking is generated using the fundamental beam itself. If the effect of the plasma on the transverse mode exhibits dynamic behavior, this might lead to error signal distortions. A solution to this problem might be the use of an intermediary single-frequency continuous-wave $(\mathrm{CW})$ laser, which is coupled into the cavity in addition to the seeding frequency comb [96]. Locking both the cavity and one seeding laser parameter to this CW reference promises several advantages. First, the SNR of the error signal is dramatically increased due to the confinement of the laser power generating the error signal to a single frequency [97]. Second, SPM will not affect the CW beam. And third, the fluctuations of the spatial profile of the resonant $\mathrm{CW}$ beam will carry information on the time scale(s) of plasma dynamics ${ }^{2}$.

\subsubsection{XUV Output Coupling}

Finding a suitable output coupling solution has been one of the main tasks within the HHG enhancement cavity community over the past few years and also a subject of this thesis. The strict design criteria of an XUV output coupler makes this task highly challenging. This challenge is described in the first part of Section 2 of the paper enclosed [35] (on page 12110) in Chapter 6. The results regarding output couplers obtained during the work that led to this thesis are discussed in Section 4.4. An outlook on the development of XUV output coupling techniques planned in our group is provided in Section 5.2. An overview of the methods proposed and demonstrated so far is given in Appendix 7.3 .

\footnotetext{
${ }^{2}$ Probing the negative plasma lens to investigate whether the plasma decays between the pulses or not, could in principle be also done by sending a single-pass beam trough the focus and observing its phase front distortions.
} 
Lehrling ist Jedermann.

Geselle, der was kann.

Meister, der was ersann.

Alter Handwerkerspruch

\section{Chapter 4}

\section{Experimental Setup and Results}

\subsection{Yb-Based CPA System with $80 \mathrm{MHz}, 200 \mathrm{fs}, 50 \mathrm{~W}$}

The laser system providing the pulses for our enhancement cavity was designed and built by the fiber laser group from the Institute of Applied Physics at the University of Jena. The main components, the mode of operation and the performance are described in detail in 98 . This section concentrates on the adaptations made to the laser system, which were necessary for the operation in conjunction with our enhancement cavity.

\subsubsection{The Oscillator}

The $78 \mathrm{MHz}$-repetition-rate pulse train is delivered by an Yb:KYW solid-state bulk crystal based, passively mode-locked oscillator, depicted in Fig. 4.1. The crystal is optically pumped by a Jenoptik $\mathrm{CW}$ laser Diode providing $2.2 \mathrm{~W}$ of average power at a central wavelength of $980 \mathrm{~nm}$ for a pump current of $3.5 \mathrm{~A}$. The central wavelength of the transferlimited $170 \mathrm{fs}$ pulses is $1042 \mathrm{~nm}$. The average output power of the oscillator for a diode pump current of $3.5 \mathrm{~A}$ is $150 \mathrm{~mW}$. This value deviates from the $220 \mathrm{~mW}$ reported in [98] due to the changes made to the oscillator, which are addressed in the following.

As discussed in Section 2.1.3, for resonant enhancement the two independent frequency comb parameters need to be set in the vicinity of the optimum overlap with the enhancement cavity resonances. Then, at least one of these parameters needs to be actively locked using a feedback loop. We manually vary the oscillator cavity dispersion by displacing a fused silica wedge in the beam. A second wedge is used to account for the direction change of the beam passing through the first wedge, see Fig. 4.1. The angle of incidence on the wedge surface is close to Brewster's angle to ensure low losses for p polarization. Varying the roundtrip dispersion of the oscillator cavity changes the difference between the phase and the group velocity and thus, influences $\omega_{\mathrm{CE}}$ and the carrier-envelope offset slippage, cf. Section 2.1.3 an in particular eq. 2.4). In addition, we can vary the current driving the pump diode to influence the laser parameters. An active control of this degree of freedom is not installed yet. However, it could be implemented in future for a fast stabilization of the second comb parameter see e.g. [52,53, 65]. The position of the wedge as well as the pump 


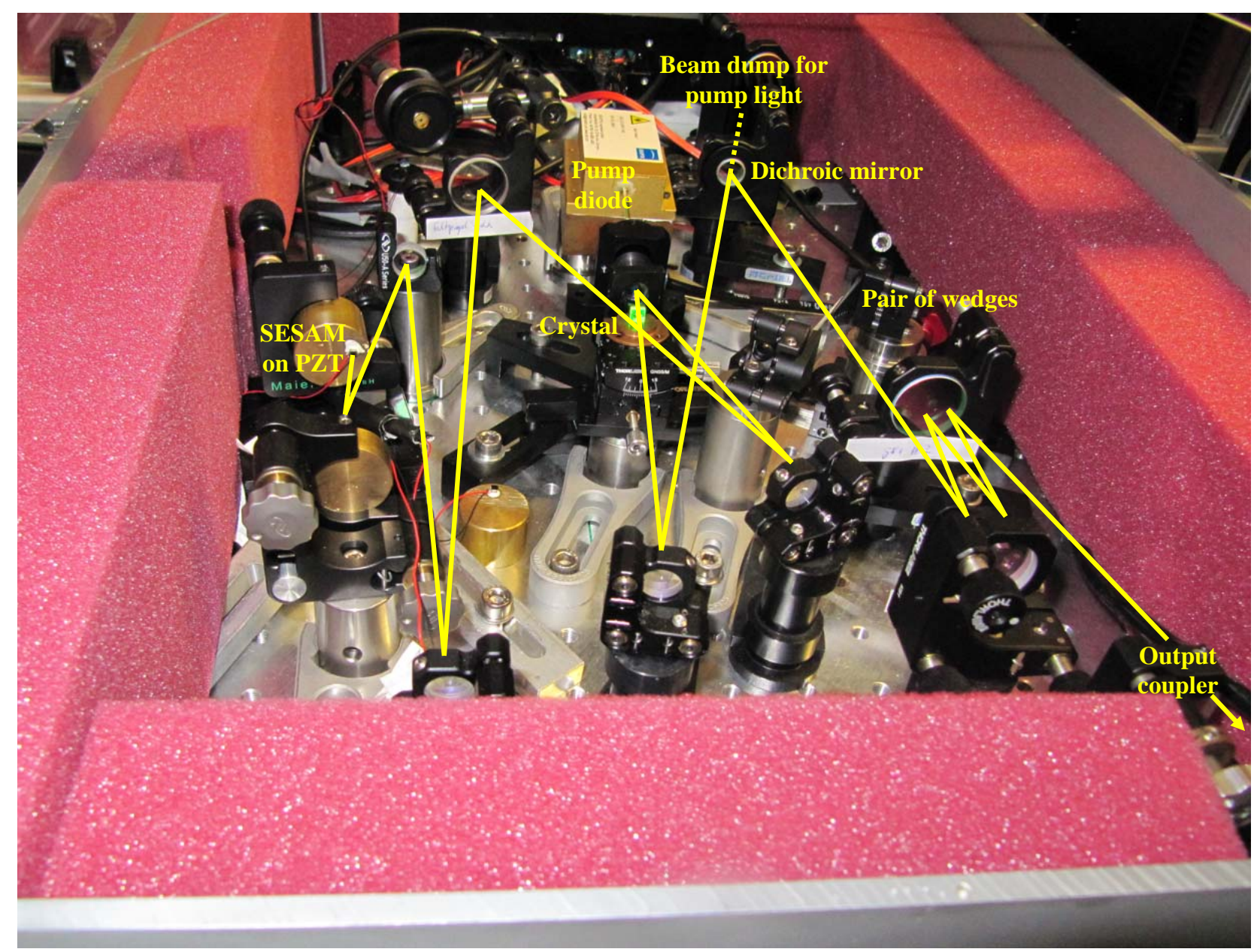

Figure 4.1: Oscillator picture (see text for details).

current are both set manually. Once the seeding frequency comb is brought in the vicinity of the optimum overlap with the cavity resonances by means of at least one of these two mechanisms, we lock the laser to the cavity with a fast piezo-electric transducer (PZT), onto which one of the cavity end mirrors is glued. We found empirically that the amplitudes of the drifts of the two laser parameters are small enough so that stable locking over several minutes can be performed with a single parameter, as described in Section 2.1.3.

The mirror used for the active lock is a semiconductor saturable absorbing mirror (SESAM, provided by Amplitude Systems), which is also responsible for the mode-locking. The SESAM surface was initially $\sim 5 \times 5 \mathrm{~mm}^{2}$. We chose this mirror to implement the active stabilization because it is the thinnest (and therefore lightest) one in the oscillator cavity and also because the impinging beam diameter is the smallest of all mirrors. The SESAM was cut into 4 equal square pieces, one of which was glued on a fast PZT (PI-PL022.31), see Fig. 4.2. The opposed surface of the PZT was glued onto a bulky brass recoil body to avoid mechanical resonances of the system at acoustic frequencies. The maximum travel of 


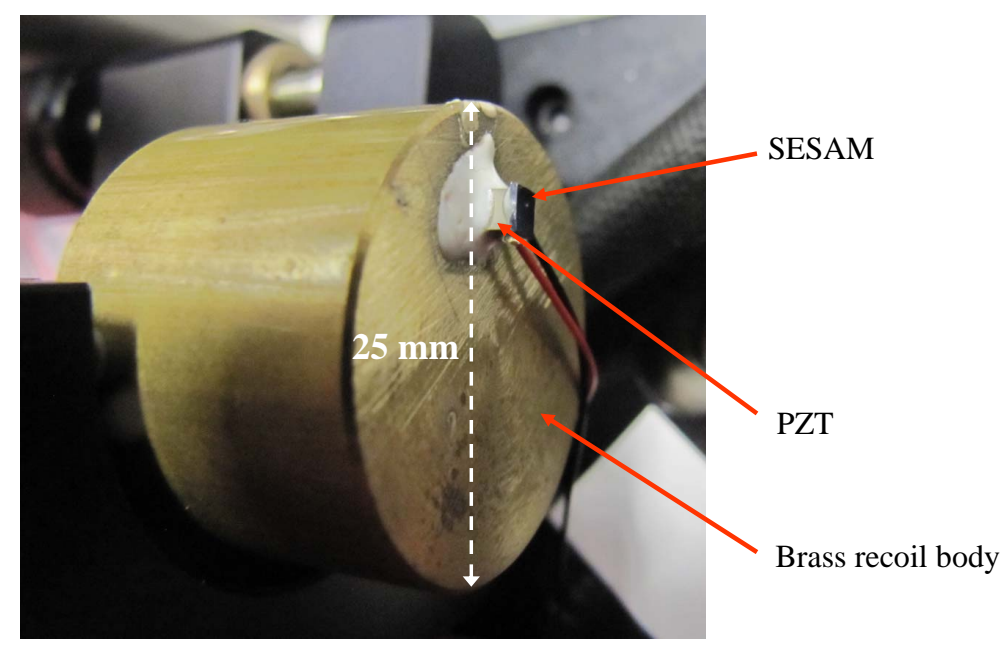

Figure 4.2: SESAM-PZT-recoil body construction.

the PZT is $2.2 \mu \mathrm{m}$. To ensure that the PZT system does not have mechanical resonances at low acoustic frequencies, we applied a sinusoidal signal to the PZT and slightly misaligned the oscillator cavity, so that a PZT travel resulted in an amplitude change of the oscillator output signal. We swept the frequency of the driving sinusoidal signal from a few $\mathrm{Hz}$ to many $\mathrm{kHz}$ and observed the phase shift of the oscillator output modulation with respect to this signal on an oscilloscope. Figure 4.3 shows oscilloscope screenshots for $100 \mathrm{~Hz}$ and $29 \mathrm{kHz}$. While at low frequencies the oscillator output amplitude modulation follows the PZT driving signal with a constant phase, at roughly $30 \mathrm{kHz}$ it exhibits a phase shift of approximately $\pi$ indicating the presence of a mechanical resonance. The phase shift between the two signals gives a good hint at the presence of mechanical resonances so that this method can be applied to roughly determine the linear range of the mechanical system's transfer function. In this case we can assume a linear response up to approx. $20 \mathrm{kHz}$. While our locking loop performs satisfactorily for enhancement factors of just below 2000, the system is very sensitive to acoustic noise and vibrations in this highfinesse operation regime. An improvement of the lock stability is one of the near-term goals in the further development of this system. To increase the available lock bandwidth, new mirror-on-PZT systems could be used, such as the recently demonstrated system with a linear bandwidth in excess of $180 \mathrm{kHz}$, see [99].

The oscillator was empirically found to be the most critical component in terms of mechanical stability. Therefore, additional measures were taken to avoid both fast and long-term drifts, cf. Fig. 4.1. To suppress sound waves inside the oscillator box, we attached absorption panels (Axifoam SH001, $50 \mathrm{~mm}$ ) to the inner side of the surrounding box walls. Moreover, to avoid a feedback of the pump diode light, a dichroic mirror was used in the resonator. The transmitted pump light was dumped onto a bulky beam dump for thermal stability. 

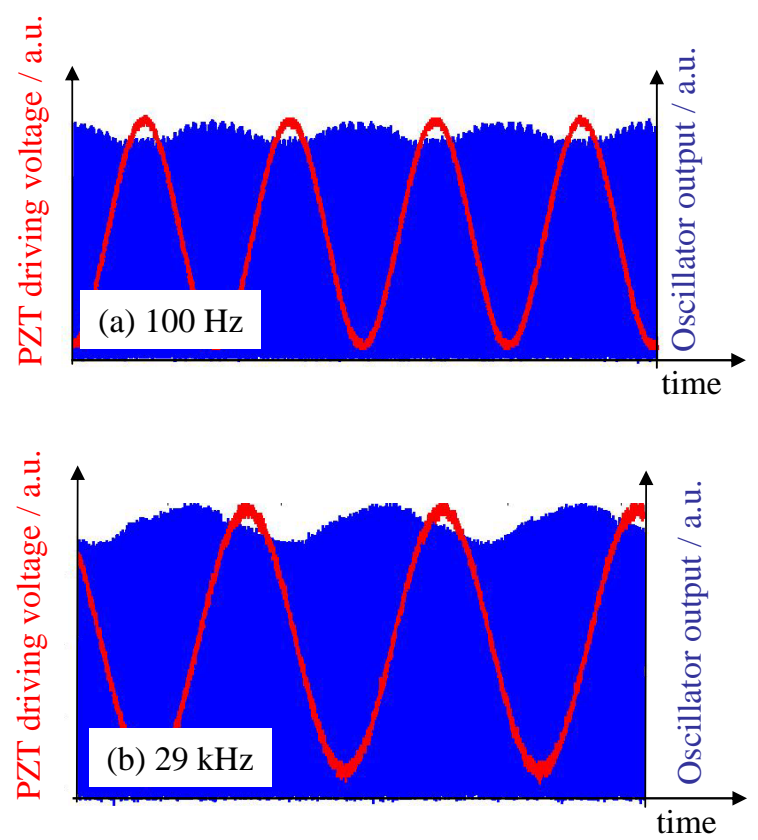

Figure 4.3: Bandwidth measurement of the PZT construction shown in Fig. 4.2, performed by a misalignment of the oscillator so that (a) the output beam is modulated according to the displacement of the PZT. Red: PZT driving voltage, blue: oscillator output. (b) A phase shift of $\sim \pi$ is observed around $30 \mathrm{kHz}$, indicating the first mechanical resonance.

\subsubsection{Chirped-Pulse Amplification}

The pulses generated by the oscillator are stretched to $150 \mathrm{ps}$ in a transmission-grating based stretcher (1250 lines $/ \mathrm{mm})$ with an efficiency of $55 \%$. Subsequently, the chirped pulses are amplified in a two-stage fiber amplifier using polarization-maintaining photonic-crystal fibers, see [98]. The amplified pulses are compressed with two highly efficient fused silica transmission gratings with 1250 lines/mm [100]. The output pulse parameters are $200 \mathrm{fs}$ and up to $60 \mathrm{~W}$ of average power. The deviation with respect to the parameters published in 98 is due to the better alignment of the oscillator, resulting in a broader bandwidth and due to several exchanged components, including the fibers and the pump diodes.

By varying the distance of the two compressor gratings, the output pulse duration can be adjusted continuously between the close-to-transfer-limited value of $200 \mathrm{fs}$ and several ps while leaving the other output parameters constant. The dependence of the spectral phase on the grating distance can be found e.g. in Appendix A.2 of [101]. The straightforward variation of the output pulse duration over a large range is particularly important for peak power scaling experiments at constant average powers. 

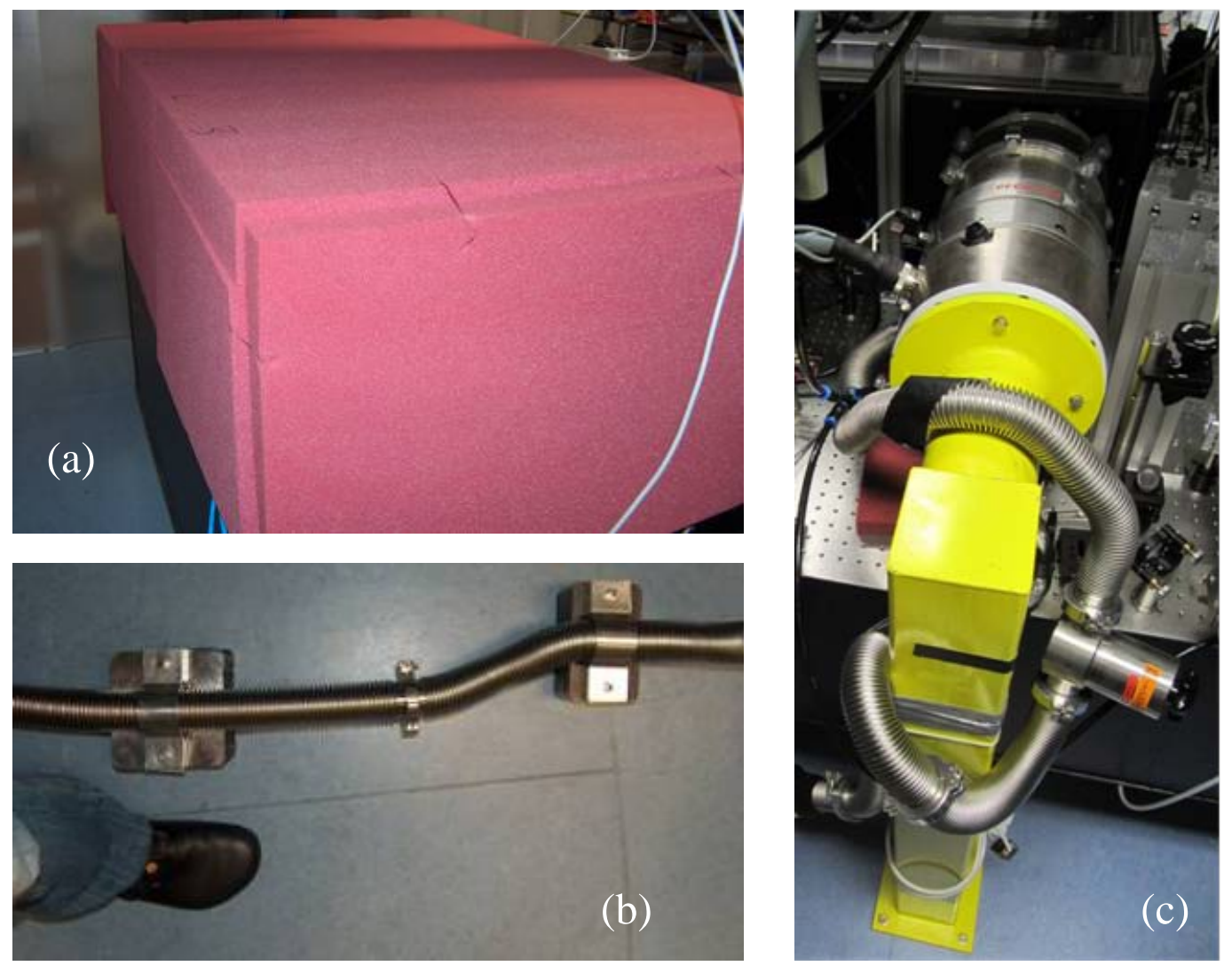

Figure 4.4: (a) protection housing of the laser against mechanical vibrations and air fluctuations. (b) vibration-damping heavy iron blocks attached to the bellows connecting the pre-vacuum pump with the turbo pump. (c) mounting of the turbo pump and connection to the vacuum chamber containing the enhancement cavity (see text for details).

\subsection{The Enhancement Cavity}

\subsubsection{Setup}

The enhancement cavity is described thoroughly in the papers included [33, 34, 37], see Chapter 6. In this section the description is rounded up with additional details on the passive protection of the setup against mechanical vibrations and heating. Measures against these factors include the use of rigid components (base plates, mounts etc.) i.e. with large inertia, the use of oscillation damping mounting posts and a housing around the laser system, doubled with absorption panels (Axifoam SH001, $50 \mathrm{~mm}$ ), see Fig. 4.4.a. The latter also prevents air fluctuations. Scattered and residual light is dumped using cooled beam dumps to avoid heating of the system components which might lead to instabilities. Despite these measures, a long-term drift of the relative length between the seeding oscillator and the enhancement cavity can be observed. Its origin is subject to current investigation. 
The vacuum pumps attached to the chamber containing the cavity constitute an unavoidable source of vibrations and we took several measures to damp these vibrations before they reached the vacuum chamber. The pump building up the pre-vacuum (Busch Fossa 0030A/B scroll pump) is attached to a turbomolecular drag pump (Pfeiffer TMH 1000M) with $\sim 4 \mathrm{~m}$ long flexible bellows. Several heavy iron blocks are attached to the bellows to damp the vibrations caused by the pre-pump, see Fig. 4.4.b. A vibration damping bellows is mounted between the turbo pump and the vacuum chamber. The pump itself is fixed to an iron pillar attached to the floor, see Fig. 4.4.c. The vacuum chamber walls are also mechanically detached from the base plate of the enhancement cavity so that the coupling of vibrations from the vacuum pumps is minimized.

\subsubsection{Diagnostics}

The diagnostics used to characterize the intracavity beam are addressed in the papers included [33, 34, 36, 37], see Chapter 6. This section contains additional details.

The intracavity beam is mainly monitored by observing the leakage through the highly reflecting cavity mirrors. The devices used to this end are: a power meter (Coherent OP-2 IR, FieldMate), two optical spectrum analyzers (Ocean Optics HR 4000 and ANDO AQ 6315A), an autocorrelator (APE PulseCheck), a photodiode (Hamamatsu InGaAs G8376) and a CCD camera (WinCam D). The transmission of the cavity mirrors used for diagnostics amounts to $1.65 \cdot 10^{-6}$ when measured with an incident power of $50 \mathrm{~W}$ (estimated uncertainty of $10 \%$ ). The transmission and reflectivity are nearly constant over the entire bandwidth of the enhanced pulses. However, when many $\mathrm{kW}$ of power circulate inside the cavity, this transmission value might change due to effects caused by the large powers. Thus, in order to prevent systematic errors in the intracavity beam characterization, we implemented a second measurement method of a different nature to the first one which uses the leakage beam. In the cavity around the laser beam, we placed a circular aperture with a diameter around 3 times larger than the $1 / e^{2}$ beam diameter (see Fig. 1.b on page 2052 of the paper included [33]). This aperture is realized as a hole in a reflector. It clips the beam and sends the clipped portion to a detector. In theory, the beam intensity profile after one cavity round-trip behind this aperture (i.e. the far field of the aperture) corresponds to the convolution of the Fourier transform (FT) of the beam profile just before the aperture with the FT of the aperture function (Fraunhofer diffraction). With the aperture being very large in respect to the beam size, the FT of its function can be approximated by a very narrow peak, so that the beam profile remains largely unaffected by this convolution. In practice, we choose the diameter empirically as small as possible under the constraint that the enhancement is not significantly affected by the aperture. Even though the clipped portion of the beam is insignificant for the cavity enhancement, owing to the large circulating powers, it is well measurable. Since the ratio of the powers measured with both methods (i.e. leaking portion and clipped portion) stayed constant throughout the power scaling experiments [33], we assume that all measurements using the leakage beam are reliable. 


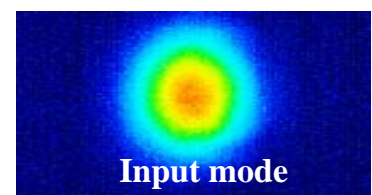

(a)

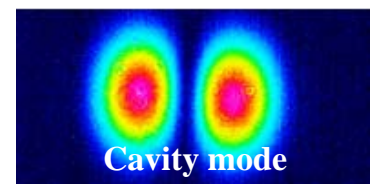

(b)

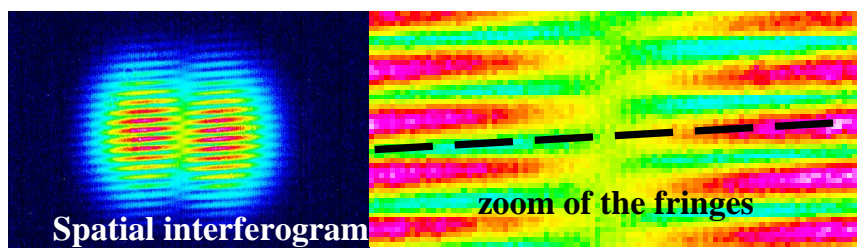

(c)

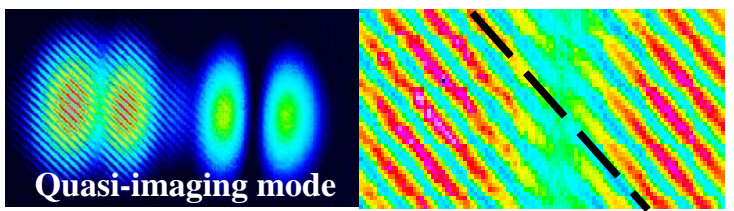

(d)

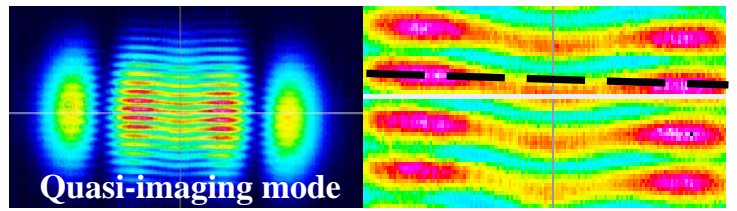

(e)

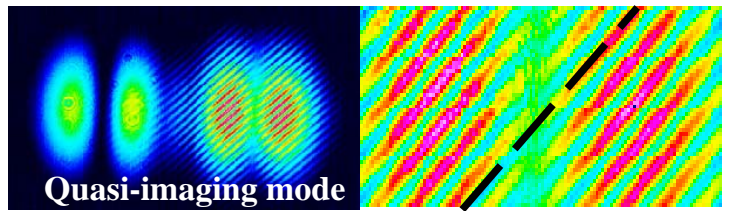

(f)

Figure 4.5: Spatial interference of a copy of the input beam (a) with higher-order modes, detected on a CCD camera; (b) the cavity TEM 10 mode; (c) spatial interferogram of the beams in (a) and (b). The dashed line indicates that the lobes of the excited mode are anti-phased; (d)-(f) interferograms of a quasi-imaging mode. The two central lobes are in phase (e) while the first two (d) and the last two (f) lobes are anti-phased.

A diagnostic method, which we developed in the course of this work is the cavity roundtrip dispersion measurement via spatial-spectral interferometry of a copy of the seeding laser beam with a copy of the intracavity circulating beam. The method is described in the paper included [34], see Chapter 6. By exchanging the imaging spectrometer with a CCD camera, the interferometer is also suited for the detection of the spacial phase relation between the lobes of higher-order modes excited in the cavity. Examples are shown in Fig. 4.5. For best fringe contrast, the delay of the two interferometer arms was set to 0 .

One of the most useful diagnostics for the alignment of the system is the periodic scan of the seeding comb through the cavity resonances, see also Section 2.1.4. Ideally, the scan time over a resonance should be chosen much longer than the cavity build-up and ringdown times, so that at each point in the scan a close-to-stationary state can be assumed. However, unless the relative positions of the comb and the cavity are actively locked during this scan, in a real experiment fluctuations are likely to distort the scan pattern if the scan speed is too slow. This holds in particular for high-finesse cavities with long build-up times. Therefore, for the diagnostic methods described in the following, we choose a scan speed which is large enough to prevent fluctuations from prevailing and slow enough to allow for a clear distinction of peaks with different magnitudes in the scan pattern. In our system, usually, the scan is performed with a triangle signal with $20-\mathrm{Hz}$ period and a PZT excursion of up to $2.2 \mu \mathrm{m}$. 


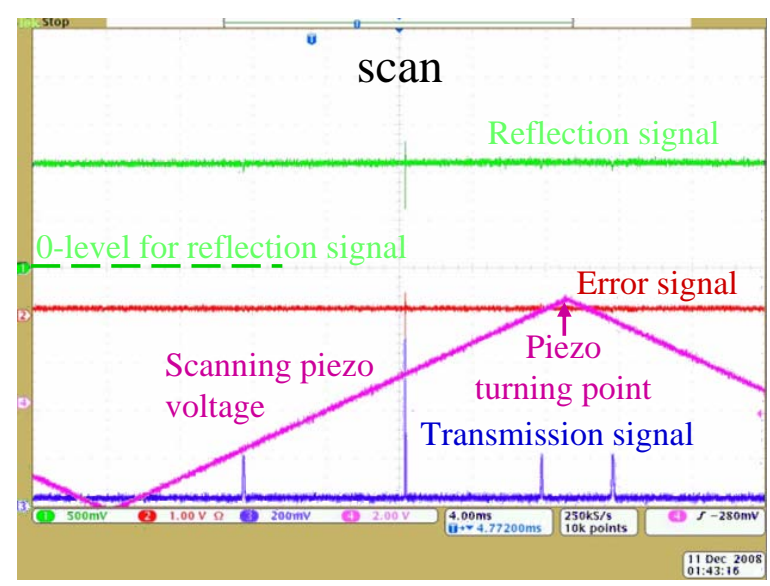

(a)

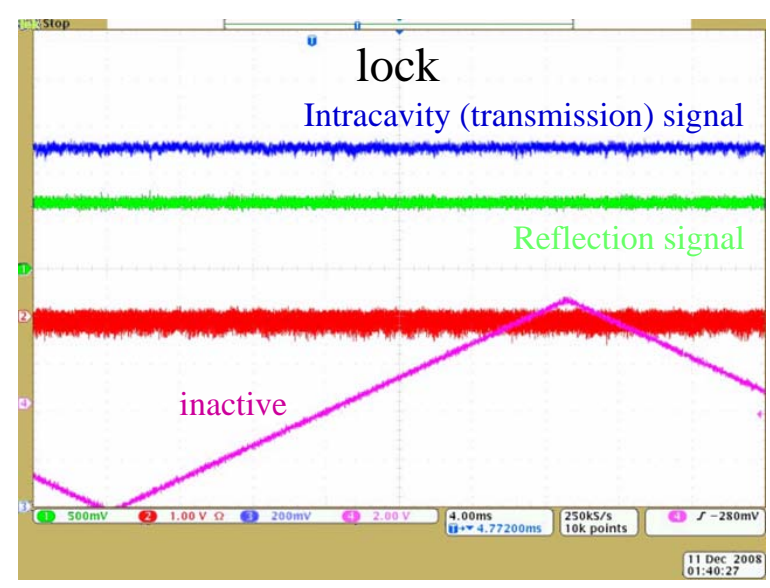

(b)

Figure 4.6: Oscilloscope screenshots of: (a) cavity length scan (amplitude slightly larger than $2 F S R$, period $20 \mathrm{~Hz}$ ). The central peak corresponds to the optimum overlap of the comb modes with the cavity resonances. The peaks to the left and to the right correspond to the cases in which the comb is shifted by approx. one FSR with respect to the optimum overlap by means of the repetition rate (secondary resonances). Due to good transverse mode matching, no light is coupled to higher-order transverse modes. (b) The locked state. Here, an effective power enhancement of 700 was achieved.

As mentioned in Section 2.1.4, the intracavity intensity evolution while scanning the frequency comb over the cavity resonances (i.e. the scan pattern) is a powerful diagnostic. The scan pattern can be used to monitor changes of the cavity finesse, the enhanced bandwidth, the detuning from the stability range center and the coupling to higher-order modes, all of which is particularly useful in power scaling experiments.

Figure 4.6. a shows an oscilloscope screenshot of a scan over slightly more than two FSR. The PZT in the oscillator cavity is driven periodically with a voltage which is proportional to the purple signal. The blue signal indicates the intracavity power, measured with a photodiode through one of the cavity mirrors. The central (highest) peak corresponds to the main resonance, i.e. the case of optimum overlap of the seeding comb with the cavity resonances. The secondary peaks to the left and to the right are generated by a comb-cavity overlap which is reached when the change of the repetition rate is large enough so that it has a similar effect to an overall shift of the frequency comb of approximately one cavity FSR. However, these peaks are smaller and broader than the main resonance peak due to the repetition rate mismatch. Increasing the repetition rate (i.e. cavity length) mismatch further leads to further secondary resonances. The corresponding peaks exhibit decreasing amplitudes and increasing widths as the mismatch grows. The ratio of the maxima of the main resonance peak to the secondary resonances increases with the coupled bandwidth. In the case of a CW laser, all resonances have the same hight. 


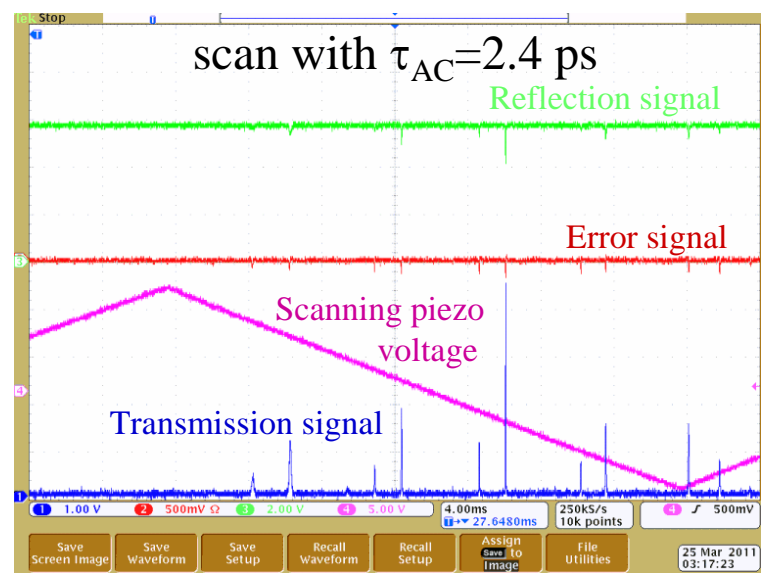

(a)

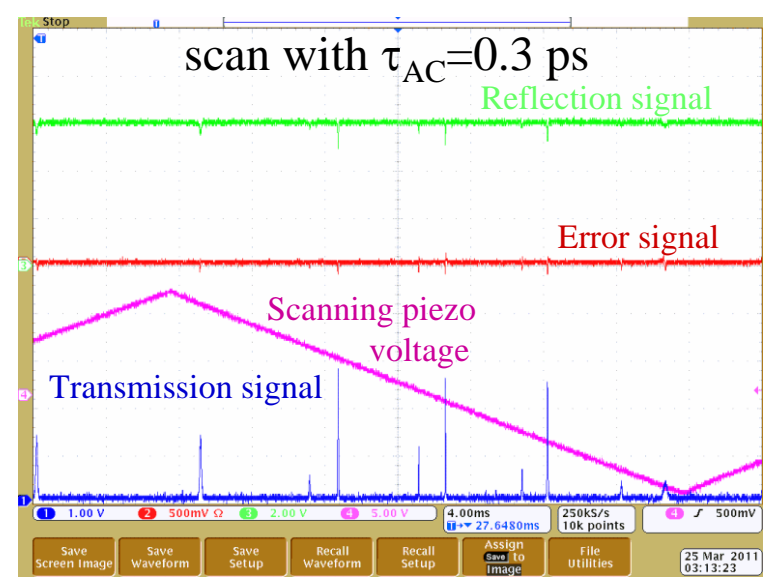

(b)

Figure 4.7: Oscilloscope screenshots of the scan patterns with a 1-mm thick fused silica intracavity Brewster plate, for an input power of $35 \mathrm{~W}$ and two different pulse autocorrelation durations: (a) $\tau_{\mathrm{AC}}=0.3 \mathrm{ps}$ and (b) $\tau_{\mathrm{AC}}=2.4 \mathrm{ps}$. The peaks corresponding to higher-order modes are clearly visible, which indicates a suboptimal alignment. The changing peak maximum ratio of the central peak to the next secondary peaks confirms the nonlinear effects observed in the other measurements (see text).

Figure 4.7 shows two scan patterns which were recorded with a 1-mm thick fused silica intracavity Brewster plate. Except for the input pulse duration, all seeding beam parameters are kept the same in the measurements in panels (a) and (b). The fact that the central peak (main resonance) is significantly weaker for input pulses with an autocorrelation duration of (a) $\tau_{\mathrm{AC}}=0.3 \mathrm{ps}$ than for (b) $\tau_{\mathrm{AC}}=2.4 \mathrm{ps}$ clearly indicates nonlinear effects limiting the intracavity peak power.

The scan pattern is also an indicator for the transverse mode matching and the alignment. For example, the alignment which led to Fig. 4.6. a is very good, since higher-order modes are barely visible. In contrast, intensity peaks corresponding to higher-order-modes are visible in the scan patterns shown in Fig. 4.7, indicating a misalignment either of the input beam or of the cavity (see also Section 4.4.1).

Moreover, the scan pattern can be used to coarsely adjust the two laser parameters and the cavity parameter in a vicinity of the optimum overlap, so that the active feedback $\operatorname{loop}(\mathrm{s})$ can subsequently provide a locked steady state. Firstly, it is necessary that the main peak lies in the scan range of the PZT. This can be adjusted by manually setting the relative length of the oscillator and the enhancement cavity. Secondly, the other laser parameter can be coarsely adjusted (e.g. by means of the wedges in the oscillator cavity or the pump power of the oscillator). In our case, the empty cavity dispersion over the enhanced optical bandwidth is small enough so that it does not affect the circulating pulse significantly. In this case, optimum overlap is achieved when the central peak is maximized and, in a good approximation, the closest secondary resonances have equally high peaks. For example, this is the case in Fig. 4.6.a but not in Fig. 4.7.a. 


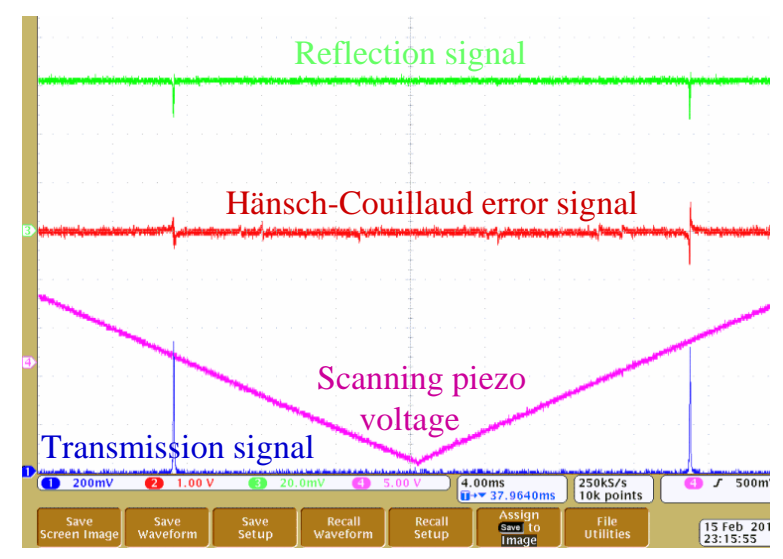

(a)

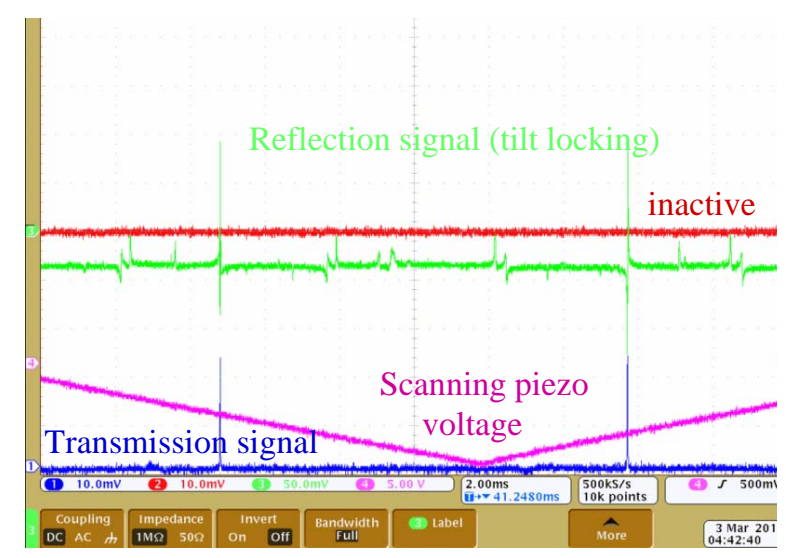

(b)

Figure 4.8: Oscilloscope screenshots of: (a) cavity length scan. The seeding transverse mode is matched to the excited cavity mode so that the reflection signal has a dip shape. In contrast, the Hänsch-Couillaud error signal exhibits a slope when passing through the cavity resonance, whose sign indicates the scan direction (bipolarity). (b) By introducing a transverse mode mismatch of the seeding beam with respect to the cavity mode, the reflection signal (offset for clarity) can be used to produce a bipolar error signal (see text).

Another important scan pattern signal monitors the reflection of the input beam from the cavity input coupler. As the frequency comb approaches optimum overlap during the scan, light is coupled to the cavity, which results in an intensity dip in the reflection signal, cf. Figs. 4.6.a, 4.7 and 4.8.a. The dip depth and shape primarily depend on the transverse mode matching, the spatial region of the beam profile which is used to generate the signal, the scan speed and the impedance matching. Let us first assume perfect transverse mode matching, see e.g. Fig. 4.8.a. The scan speed determines the time the light has to build up in the cavity and hence, influences the hight of the transmission signal peak(s) and the depth of the reflection dip(s), thereby giving a measure of the amount of light coupled into the cavity. The dependence of the dip depth on the scan speed relates the build-up process to the scan time and could in principle be used to determine the degree of impedance matching of the cavity. However, we have not done this so far. On the other hand, we frequently use the reflection signal to maximize the dip depth for the coarse setting of the laser and cavity parameters in order to achieve the desired vicinity of the optimum overlap. It is noteworthy that like the transmission signal, the reflection signal in the case of perfect transverse mode matching does not provide a bipolar signal which could be used as an error signal for active stabilization, see e.g. Fig. 4.8.a. However, if a slight transverse mismatch of the input beam with respect to the cavity mode is introduced, like a tilt or beam parameter mismatch, then a portion of the reflected beam having a bipolar shape on resonance can be spatially filtered for detection. The reason for this shape is the interference of the reflected part of the input beam that is resonant with the cavity 
mode (and therefore bears the information on the cavity roundtrip phase) with a portion of the input beam which is spatially filtered out, i.e. rejected by the cavity, and travels along a slightly different path which introduces a delay towards the first part and acts as a reference. This leads to a transverse mode mismatch locking error signal as can be seen in Fig. 4.8.b.

Although the above mentioned method was successfully employed to lock the cavity, for most of the experiments presented in this thesis we used the Hänsch-Couillaud locking scheme. Figure 4.8. a shows a typical Hänsch-Couillaud signal. With such a signal and an alignment leading to a scan pattern similar to Fig. 4.6.a, a stable lock over several minutes can be achieved, see Fig. 4.6.b. The scan pattern is also useful for balancing the difference photodiode in the Hänsch-Couillaud scheme as well as the amplitude of the error signal. The transmission signal level difference of the main peak in Fig. 4.6. a and the locked level in Fig. 4.6. b is due to the scan speed, which at this finesse does not allow for a full build-up of the main resonance. For locking we used a customized proportional integral controller (Menlo Systems Lockbox PIC 201).

Further diagnostics customized during this work include a z-scan setup and a ringdown loss-meter, adapted for arbitrary angles of incidence. These devices are described in the paper included [36], see Chapter 6. The vacuum z-scan setup can be used for nonlinear refractive index measurements (cf. 102,103]) and as a single-pass diagnostic for nonlinearities in cavity optics. It was developed with Jan Kaster, who also constructed it.

\subsection{Power Scaling of the Empty Cavity}

One of the major results of this work consists in the power scaling experiments performed with the standard-design bow tie cavity presented in Section 4.2.1 and is documented in the paper enclosed [33], see Chapter 6. The significance of these experiments is twofold. On the one hand, they show that the enhancement cavity technique enables the combination of high pulse energies (up to the the mJ level) with high repetition rates $(80 \mathrm{MHz})$ for sub-ps pulses with standard optics. This power regime is ideally suited for driving low-conversionefficiency nonlinear processes and cannot be achieved with alternative techniques to this day. On the other hand, these experiments reveal power scaling limitations of standard bow-tie-design enhancement cavities built with state-of-the-art mirrors. Identifying these limitations allows for the development of new solutions to overcome them. In particular, the high-intensity induced nonlinear effects on the mirrors have been identified as one of the main power scaling limitations. As a consequence, our group is developing a novel cavity design supporting increased spot sizes (thus decreasing the intensity ) on the mirrors, see Section 5.1.1. Peak power scaling limitations are identified also in the experiments reported in Section 3.2 of the paper enclosed [34], see Chapter 6. By using the novel spatial-spectralinterferometry-based technique, an intensity-dependence of the empty cavity roundtrip $G D D$ was measured. 


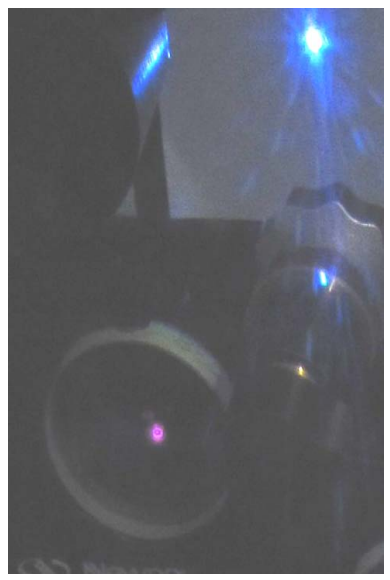

(a)

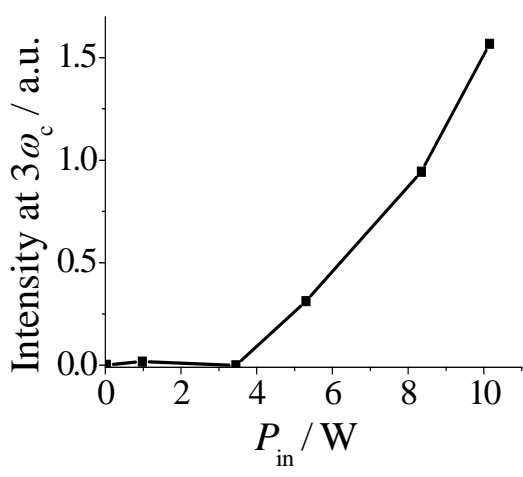

(b)

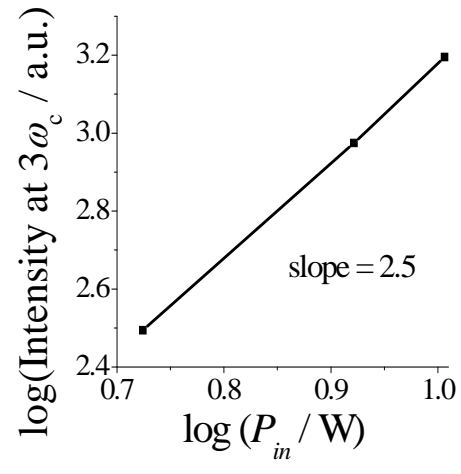

(c)

Figure 4.9: Third-harmonic generation at the cavity mirrors: (a) photograph of cavity mirror surface and of the fluorescence on a piece of paper placed behind the mirror; (b) UV spectrometer counts [a.u.] at $347 \mathrm{~nm}$ versus input power $P_{\text {in }}$ at a constant input pulse duration of $200 \mathrm{fs}$; (c) log-log plot of the points, at which THG was detected. The slope indicates a 2.5-power dependence, see text.

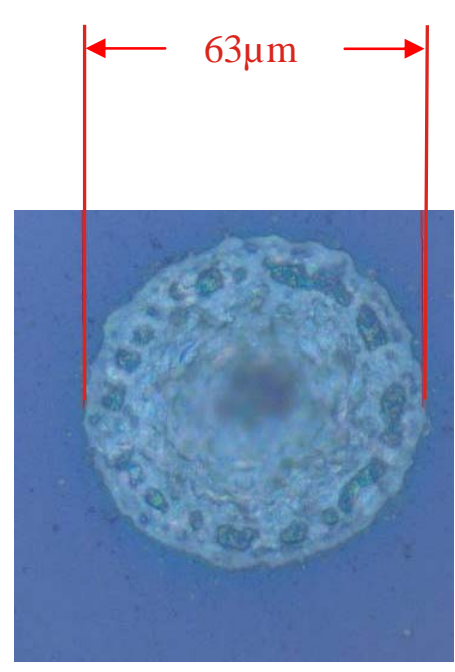

(a)

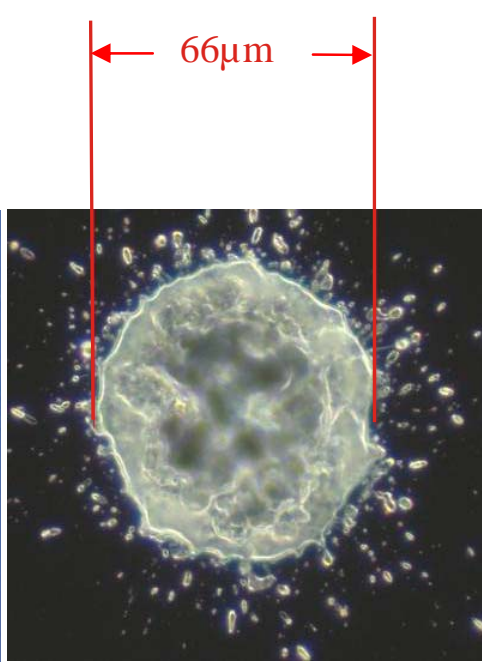

(b)

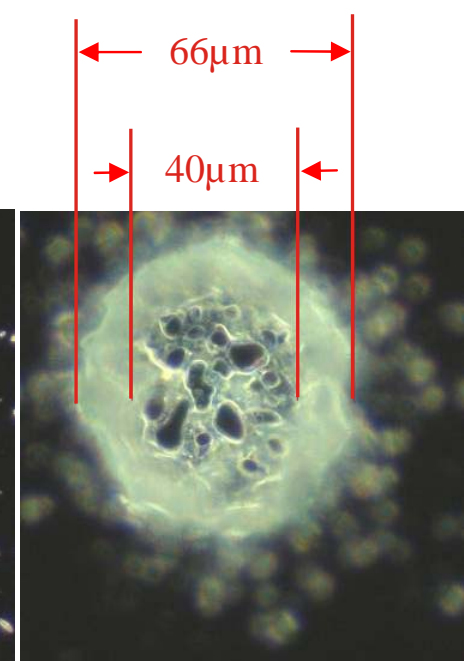

(c)

Figure 4.10: Optical microscope images of enhancement cavity mirror damages: (a) input coupler, (b) and (c) same cavity high reflector, two different focusing depths of the optical microscope. 
Another phenomenon observed at large peak powers, which is not accounted for in [33] and [34] is third-harmonic generation (THG) in the cavity mirrors. Figure 4.9. a shows a picture of a piece of paper placed behind a cavity mirror. The beam transmitted through the mirror contains a portion of UV light, which leads to fluorescence on the paper. With an UV spectrometer, this light was identified as the third harmonic of the infrared fundamental radiation. The spectrometer counts at $347 \mathrm{~nm}$ for several input power values at a constant input pulse duration of $200 \mathrm{fs}$ are plotted in Fig. 4.9.b and c. The 2.5-power exponential dependence revealed by the log-log plot in Fig. 4.9. c deviates from the expected power of 3. This deviation most likely stems from the fact that due to THG the circulating power does not scale linearly with the input power, which provides the abscissa of this measurement. Optical THG at interfaces between materials with different refractive indexes is a wellknown phenomenon 104. Most likely, the UV light is generated at the interface vacuum mirror surface and/or at the interfaces of the coating layers. Independent measurements with the z-scan setup (see Section 4.2.2) showed that a portion of this generated third harmonic is transmitted and a portion reflected by the mirror. Therefore, the measured THG signal behind the cavity mirrors is a superposition of contributions from several cavity mirrors, each with a slightly different angle of incidence and spot size, which makes tracing back these contributions an involved task 1 . The results plotted in Fig. 4.9. b show qualitatively that THG in the cavity mirrors is a potential limitation of this cavity design. This corroborates the need for novel cavity designs with large spot sizes on the mirrors, like the ones described in Section 5.1.1.

Finally, Fig. 4.10 shows mirror damages produced when driving the cavity with powers resulting in intensities beyond the identified damage thresholds. The study of these damages could perhaps be useful on the way to developing mirrors with higher damage thresholds.

\subsection{XUV Output Coupling Techniques}

Coupling out the intracavity generated XUV radiation is one of the major challenges in the field of enhancement-cavity-based HHG. In the frame of the work that led to this thesis, several existing output coupling methods were investigated and further developed and also novel techniques emerged. This section addresses this progress. A summarizing overview of the proposed and demonstrated output coupling methods is given in Appendix 7.3.

\footnotetext{
${ }^{1}$ In the frame of this thesis, the THG in the cavity mirrors was not investigated quantitatively. The main reason for this is the characteristic of the cavity mirrors at the third harmonic of the fundamental radiation. Since the employed mirrors are dielectric quarter-wave stacks, they have a strong reflection band in the vicinity of the third harmonic. However, the exact position of this band in the frequency domain depends on the mirror dispersion and also strongly depends on the angle of incidence.
} 


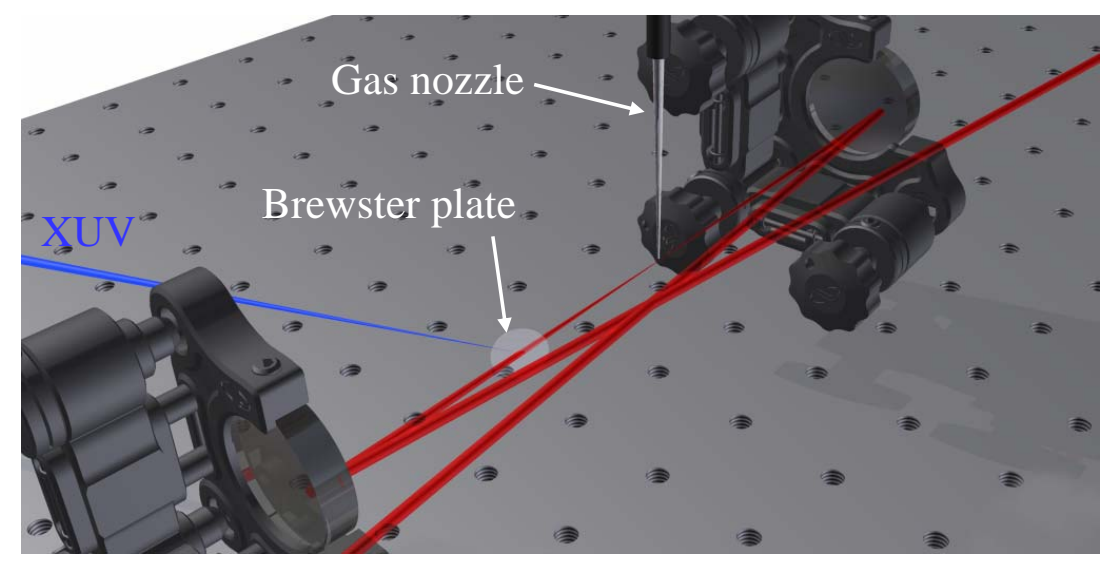

(a)

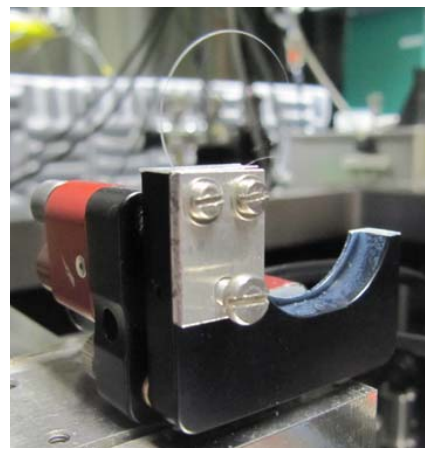

(b)

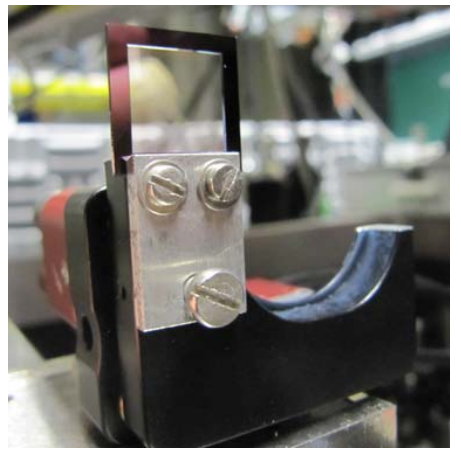

(c)

Figure 4.11: (a) The Brewster plate is located about half way between the cavity focus and the subsequent curved mirror. (b) Brewster plate mount with an $100 \mu \mathrm{m}$ thin fused silica plate and (c) with an $1 \mu \mathrm{m}$ thin $\mathrm{Si}_{3} \mathrm{~N}_{4}$ foil, mounted on a Si frame.

\subsubsection{Brewster Plates}

The first method ever employed for coupling out intracavity generated XUV radiation (and still the most widespread method) relies on a thin plate placed under Brewster's angle (for the fundamental radiation) between the HHG focus and the subsequent cavity mirror [17, 18, 20]. Since in the XUV the refractive index of the plate material is smaller than 1, the harmonic beam experiences total internal reflection at the plate surface and is coupled out of the cavity. In our setup, several thin plates/foils were tested. Figure 4.11. a $^{2}$ shows the position of the Brewster plate in the cavity and Fig. 4.11.b and c show our mount. Note that no measures for cooling the plates are taken. In our group, an extensive material search was conducted by Oleg Pronin with the purpose of identifying the materials suitable as intracavity Brewster plates. The main results of intracavity power scaling experiments with the most promising plates are summarized in Tab. 4.1 .

\footnotetext{
${ }^{2}$ The graphic illustration was done by Jan Kaster.
} 


\begin{tabular}{l|c|c|c|c|c|c|c|c} 
Material & $\mathrm{Si}_{3} \mathrm{~N}_{4}$ & $\mathrm{SiC}$ & $\mathrm{MgO}$ & $\begin{array}{c}\mathrm{C} \text { (single- } \\
\text { crystal \& } \\
\text { CVD ) }\end{array}$ & $\mathrm{Al}_{2} \mathrm{O}_{3}$ & $\begin{array}{c}\mathrm{SiO}_{2} \\
\text { crystalline }\end{array}$ & $\begin{array}{c}\mathrm{SiO}_{2} \\
\text { fused }\end{array}$ & $\begin{array}{c}\mathrm{SiO}_{2} \\
\text { fused }\end{array}$ \\
\hline Thickness & $30-500 \mathrm{~nm}$ & $300 \mathrm{~nm}$ & $50 \mu \mathrm{m}$ & $20-100 \mu \mathrm{m}$ & $340 \mu \mathrm{m}$ & $100-150 \mu \mathrm{m}$ & $100 \mu \mathrm{m}$ & $1000 \mu \mathrm{m}$ \\
\hline Maximum $P_{\text {circ }}$ & $<3 \mathrm{~kW}$ & $0.5 \mathrm{~kW}$ & $2.3 \mathrm{~kW}$ & - & $0.5 \mathrm{~kW}$ & $5 \mathrm{~kW}$ & $12 \mathrm{~kW}$ & $4.5 \mathrm{~kW}$ \\
\hline Enhancement & 685 & 50 & 500 & - & 30 (?) & 400 & 500 & $100-250$ \\
\hline Observations & $\begin{array}{c}\text { Damage at } \\
P_{\text {circ }}>3 \mathrm{~kW}\end{array}$ & $\begin{array}{c}\text { Thermal } \\
\text { effects, } \\
\text { strong } \\
\text { SHG, } \\
\text { no damage }\end{array}$ & $\begin{array}{c}\text { Thermal } \\
\text { effects, } \\
\text { damaged }\end{array}$ & $\begin{array}{c}\text { no } \\
\text { resonances } \\
\text { observed at } \\
\text { all }\end{array}$ & $\begin{array}{c}\text { SHG, } \\
\text { thermal } \\
\text { effects }\end{array}$ & $\begin{array}{c}\text { Thermal } \\
\text { effects, } \\
\text { SHG, } \\
\text { no damage }\end{array}$ & $\begin{array}{c}\text { Thermal } \\
\text { effects, } \\
\text { damage } \\
\text { after several } \\
\text { minutes }\end{array}$ & $\begin{array}{c}\text { Strong } \\
\text { nonlinear } \\
\text { effects, } \\
\text { no damage }\end{array}$
\end{tabular}

Table 4.1: Power scaling results with different intracavity Brewster plates. $P_{\text {circ }}$ denotes the circulating average power. CVD: chemical vapor deposition. Thermal and nonlinear effects are discussed in the text.

The effects limiting the power scalability of the cavity including a Brewster plate can be divided into thermal and nonlinear effects. Thermal effects can be mainly attributed to the absorption of the circulating power in the plate. However, in the results presented here, the possibility of scattered light heating up the mount of the plates cannot be excluded. Especially in the case of ultrathin foils spanned in a frame this might quickly lead to damage. To minimize the absorption in the plates, the thickness should be chosen as small as possible. However, this also implies poor heat conduction (see Section 5.2.1) and mechanical fragility. The thermal effects mentioned in Tab. 4.1 manifest themselves in two ways. Firstly, if the position in the stability range of the cavity is set so that several transverse modes are (close to) simultaneously resonant, a coupling between those modes can be observed after a "heating time" on the order of $1 \mathrm{~s}$. In other words, immediately after the cavity is locked, the fundamental, i.e. the $G H_{00}$ mode is excited and shortly after, the transverse field distribution changes, which can be detected with the CCD camera. This effect can be attributed to a phase front distortion in the plate originating either from a thermal deformation of the plate or from a temperature-dependent refractive index. Secondly, thermal effects can be identified via a "cool-down" when switching from the lock to the scan mode after a longer period of locking. Then, the initial position of the main resonance in the scan pattern (cf. Fig. 4.6) just after switching to the scan mode, changes slightly to a steady position, indicating a change of the cavity length. Both phenomena occur on a $\sim 1 \mathrm{~s}$ time scale, leading to the conclusion that they cannot be attributed to nonlinearities. For the thinner plates/foils, thermal effects eventually lead to damage. The nonlinear effects mentioned in Tab. 4.1 include mainly second harmonic generation (SHG) and self phase modulation (SPM). The latter affects the roundtrip phase of the cavity and leads to spectral filtering which can be identified in the intracavity spectrum and in the overall power enhancement. To distinguish between thermal and nonlinear effects, the input pulses can be chirped while keeping the pulse energy constant. 


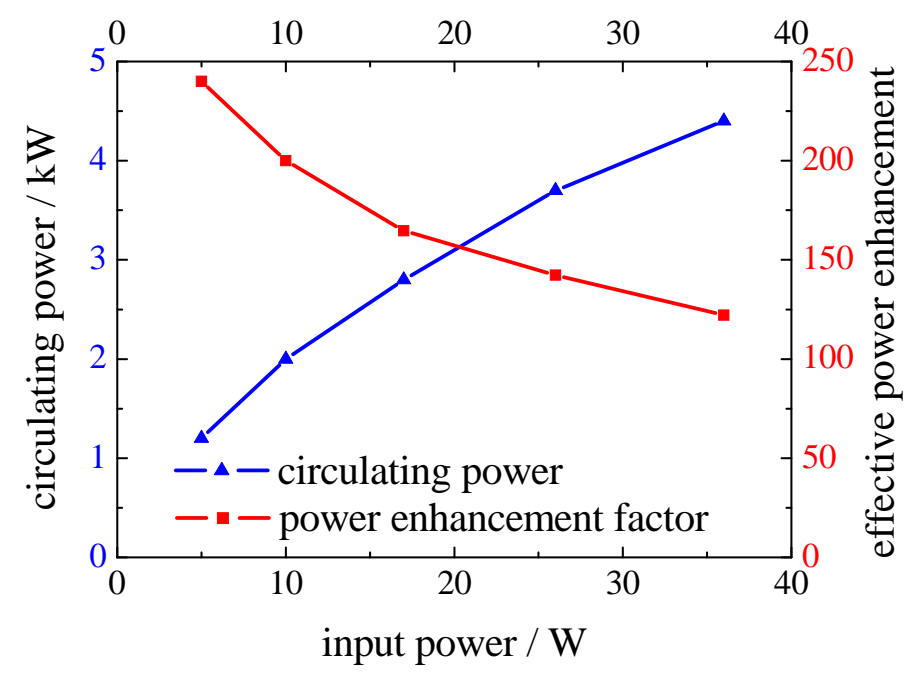

Figure 4.12: Circulating power and effective power enhancement measured as functions of the input power with a 1-mm thick fused silica intracavity Brewster plate.

\section{Fused Silica, $1 \mathrm{~mm}$}

As an example of power scaling with an intracavity Brewster plate, we discuss the results with a 1-mm thick fused silica plate. Figure 4.12 shows the measured circulating power and effective power enhancement factor as a function of the input power. The input pulse duration was kept constant (autocorrelation time $300 \mathrm{fs}$ ). A substantial power enhancement decrease is observable as the input power increases. To investigate the origin of this effect we recorded the intracavity spectrum for different input powers. Figure 4.13 shows the results. Panel (a) shows the input spectra for $5 \mathrm{~W}$ and $35 \mathrm{~W}$ at a constant input pulse duration. The power-dependent modulations in the spectrum are due to higher-order modes excited in the main amplifier fiber, which is a sign of suboptimal alignment of the input coupling in this fiber. However, this artefact does not affect the conclusions of this measurement. We observe that even at a relatively moderate power ( $5 \mathrm{~W}$, purple curve), the dispersion of the Brewster plate is not negligible since only a part of the spectrum is coupled to the cavity. Moreover, this part can be selected by varying the carrier-envelope phase slippage of the input comb (by displacing the wedges in the oscillator cavity), see green curve. As the input power is increased to $35 \mathrm{~W}$, the circulating spectrum becomes narrower, which indicates nonlinear dispersion. To ensure that the observed effects are caused by the peak power, we kept the input power of $35 \mathrm{~W}$ constant and measured the circulating spectrum for two different input pulse durations, obtained by chirping the input pulses (but keeping their bandwidth constant). The results are shown in panel (b) of Fig. 4.13. The narrowing of the enhanced spectrum for a measured input pulse autocorrelation of $300 \mathrm{fs}$ as opposed to $2.4 \mathrm{ps}$ is clearly visible and confirms the nonlinear dispersion. The scan pattern shown in Fig. 4.7 provides another confirmation of the nonlinearity (further explanations of this scan pattern can be found in Section 4.2.2. 

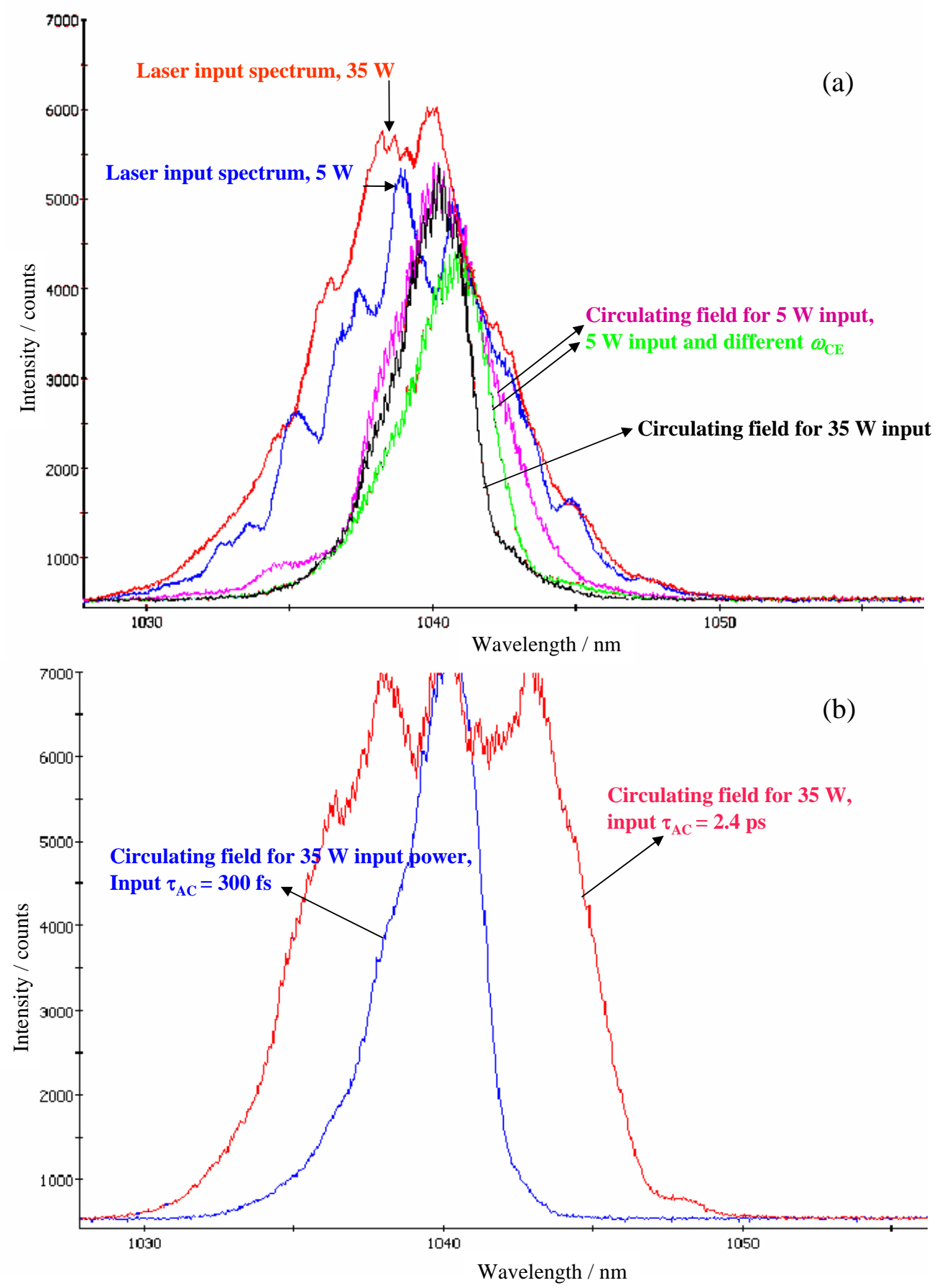

Figure 4.13: Spectra of the laser input fields and of the circulating fields for different power levels with a 1-mm thick fused silica intracavity Brewster plate (screenshots): (a) spectra for a constant input pulse duration (autocorrelation of $300 \mathrm{fs}$ ) and (b) spectra for a constant input power of $35 \mathrm{~W}$ and two different input pulse durations. (The high-frequency ripples are caused by the etalon effect of transmission neutral density filters we used to attenuate the beam. They are not related to any cavity-relevant effect.) 


\section{Fused Silica, $0.1 \mathrm{~mm}$}

The best results with a Brewster plate in our setup were achieved with a $100 \mu \mathrm{m}$ thin $\mathrm{SiO}_{2}$ (fused silica) plate. The introduced dispersion can be neglected for $200 \mathrm{fs}$ circulating pulses. With an enhancement of 500 we were able to stably lock $12 \mathrm{~kW}$ of circulating power. However, this regime could only be kept constant for $\sim 5$ minutes, after which time the plate got damaged. This was reproduced several times, using new plates. With a circulating power level of 3 to $4 \mathrm{~kW}$ long-time stable operation can be achieved, without damaging the plate. These observations agree well with a similar system reported in 23, 105]. The results presented in Section 4.5 .3 were achieved with this plate.

\subsubsection{WOMOC: Wedge-On-Mirror Output Coupler}

A novel optical element for output coupling, based on the Brewster plate, but exhibiting a better thermal conductivity was developed as part of this thesis and is described in the publication enclosed [34], see Chapter 6. A prototype of the wedge-on-mirror output coupler (WOMOC) was produced and characterized.

A further conceivable manufacturing option for a WOMOC, which is not discussed in 34 is an "upside-down" production, in which the highly reflecting multilayer structure is coated on a bulk substrate, out of which the wedge is subsequently lapped (and polished). For mechanical stability, a supporting substrate can be glued or optically contacted to the last layer of the dielectric coating before processing the upper layer.

Another advantage of the robustness of this element is that an additional (optional) heat conducting structure could be attached to the front surface of the WOMOC by coating, gluing or optical contacting. It should also be mentioned that the mirror underlying the wedge layer need not be a dielectric mirror. In principle, other mirror technologies could be used to realize the same spatial separation effect caused by the wedged layer.

\subsubsection{Direct On-Axis Access}

Ideally, the intracavity generated XUV radiation does not interact with any material while it is coupled out, a situation to which we refer as direct (or geometrical) output coupling. Two different direct output coupling techniques are being pursued in our group: (i) the enhancement of the fundamental mode with a small on-axis hole in the mirror succeeding the HHG focus and (ii) quasi-imaging (QI), i.e. the cavity operated with a combination of transverse modes, with a hole (or slit) in the same mirror.

The first approach is discussed in [106]. With the model developed there, a power enhancement of a few hundred is expected with holes with an aperture diameter of $\sim 100 \mu \mathrm{m}$ in our present setup. On the one hand, a precise prediction of the enhancement is difficult because usually the edge of such a hole is imperfect, leading to additional phase front distortions which affect the enhancement. On the other hand, manufacturing such mirrors is highly challenging. The accurate drilling of such a small hole has been demonstrated 
only recently by Dominik Esser and coworkers at the Fraunhofer ILT in Aachen, by using laser micromachining [107] (see also Section 5.2.2).

The major expected disadvantage of the first method with regard to power scaling is the fact that the field distribution of the excited cavity mode has a maximum at the position of the hole. Imperfections of the hole edge might lead to additional absorption and might decrease the damage threshold of the mirror. The quest for an alternative direct output coupling technique, which enables an intensity minimum at an aperture in the mirror following the HHG region (with an on-axis maximum) led to the development of quasi-imaging. This technique is introduced in the included publication [37], see Chapter 6, where a successful proof of principle is also presented. In Section 5.2.2 we give an outlook on the steps taken to show that this is a viable XUV output coupling method.

Due to the decreasing of the beam divergence with the harmonic order, cf. eq. 2.44 2.45), the efficiency of direct output coupling increases with the harmonic order. Moreover, since the harmonics do not interact with any material while being coupled out, we consider this to be the most promising strategy for coupling out short-wavelength XUV radiation.

\subsubsection{Other Methods}

We address two further methods which were developed in close collaboration with our project. However, these methods have not yet been implemented in our experiment.

\section{Reflective Nanograting}

A recently developed output coupler relies on a diffractive nanostructure written in the last layer of the dielectric multilayer coating of the cavity mirror following the HHG focus. The nanograting diffracts the XUV light, while the element still acts as a high reflector for the fundamental IR beam. This concept was first presented in [19]. In our group, Ying-Ying Yang and coworkers optimized, produced and characterized such an element. The results are presented in the publication enclosed [36], see Chapter 6.

A main property of the nanograting is the spatial dispersion of the output coupled high harmonics. However, this property is undesirable for several applications, such as isolated attosecond pulse generation (see e.g. Section 5.3). Moreover, in the experiments reported in [36] we have found that the nanostructure enhances nonlinear effects, which might limit the power scaling of an enhancement cavity containing such an element.

\section{GIP: Grazing-Incidence Plate}

An extension of the Brewster plate method towards larger output coupling efficiencies was developed by Oleg Pronin and coworkers [108] in our group. By applying an s-polarization broadband anti-reflection coating for large angles of incidence (e.g. $75^{\circ}$ ) on both sides of a thin transparent plate, this element can be placed in the cavity similarly to the Brewster

plate, but under a larger angle of incidence. While the output coupling efficiency is expected to be increased considerably, this approach does not overcome the inherent average (and 


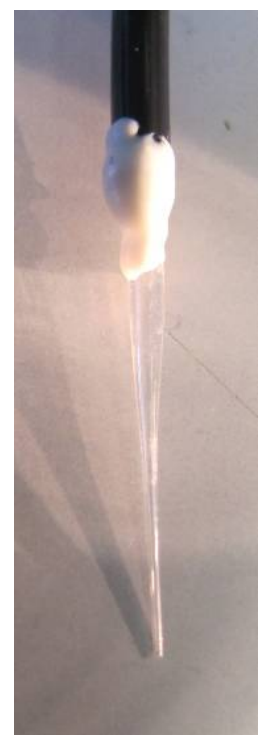

(a)

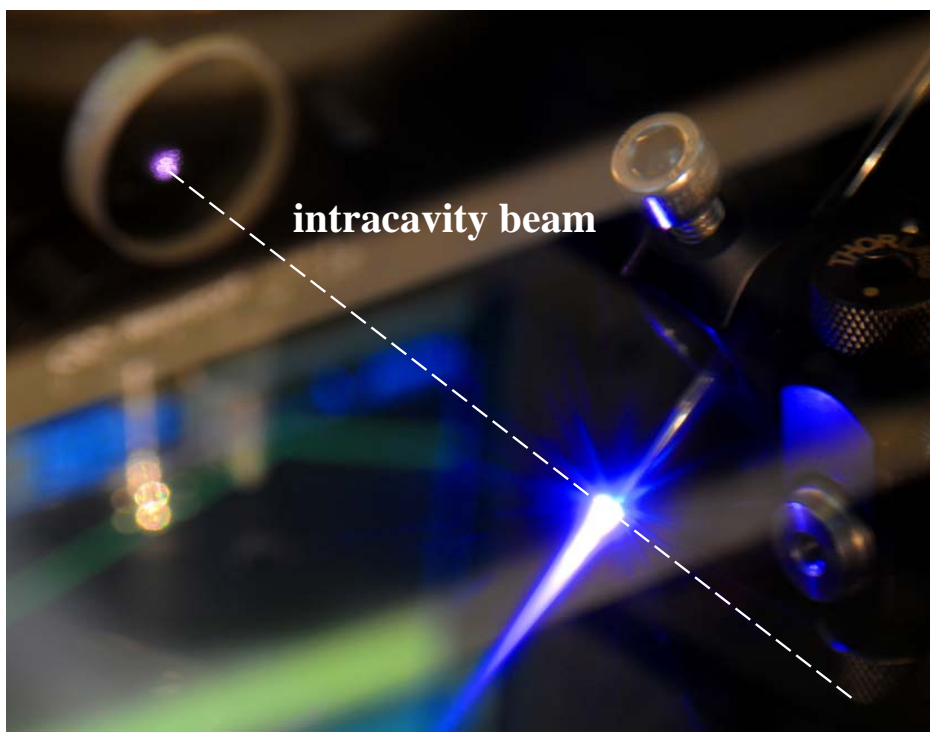

(b)

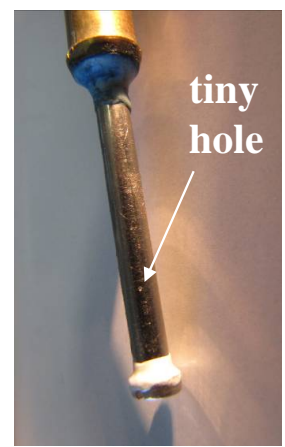

(c)

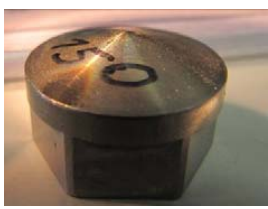

(d)

Figure 4.14: Gas nozzles for HHG: (a) A (hand-made) tapered glass capillary. Typical hole diameters lie between 100 and $200 \mu \mathrm{m}$. (b) Bright Xe plasma generated with the glass

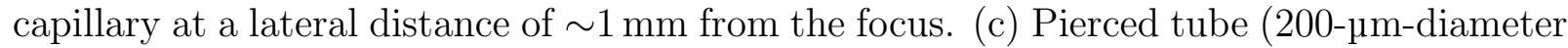
holes). (d) 150-um-diameter Laval nozzle. See text for details.

peak) power scaling limitations of the free-standing Brewster plate. In particular, the coating requires a certain substrate thickness due to mechanical tension.

\subsection{XUV Generation}

The first XUV measurements made with the system described here were carried out at relatively low powers with a conventional Brewster plate output coupler. There are three reasons for this choice. Firstly, this technique is well understood and allows the XUV detection hardware to be set up. Secondly, measurements made with the Brewster plate can be used as benchmarks for alternative output coupling methods. And thirdly, at the time of writing this thesis, none of the preferred alternative output couplers was available yet (see also Section 5.2). In the following we describe the gas target used for HHG and the XUV diagnostics, and show the first XUV spectra recorded with our setup.

\subsubsection{Intracavity Gas Target}

Figure 4.14 shows the gas nozzles available for our XUV experiments. All the experiments presented in this section were carried out with tapered glass capillaries like the one shown in Fig. 4.14 a with Xenon being used as the nonlinear medium. We chose Xe due to its low ionization potential (cf. Tab. 2.1), enabling HHG at relatively low intensities. 
Figure $4.14 . \mathrm{b}^{3}$ shows a bright plasma, generated when such a nozzle is placed at a lateral distance of approximately $1 \mathrm{~mm}$ from the cavity focus. The brightness of the plasma indicates strong ionization of the gas. On the one hand, this confirms the high intensity in the cavity focus. On the other hand, the ionization dramatically decreases the HHG efficiency. We found out empirically that moving the gas target very close to the beam increases the generated XUV power see Section 4.5.3 and [111]. In this case, the brightness of the plasma decreases significantly. The gas dynamics as well as related phase matching conditions require further intensive investigations, see also Section 3.2.2.

These handmade glass nozzles are cheap, easily produced and have several advantages regarding intracavity HHG. Their alignment is straightforward, requiring no special orientation (at least when the fundamental transverse mode is used for HHG). Moreover, they can be positioned very close to the beam. In contrast, the pierced-tube nozzle shown in Fig. 4.14.c is quite likely to clip the beam slightly due to its tiny holes, which are necessary for optimum gas flow. Owing to the large circulating power levels, even absorption of only several ppm due to clipping can lead to overheating and damage of the nozzle material, as was observed in our experiment. The use of a Laval nozzle, as the one shown in Fig. 4.14.d will be a subject of future experiments.

Without the gas target, the roughing pump alone provides a vacuum of $0.4 \mathrm{mbar}$. Switching on the turbo pump yields a pressure of $10^{-5}$ mbar. The backing pressure of the gas target can be varied up to approximately 4 bar. At a backing pressure of approx. 1.2 bar, which was empirically found to be the optimum for the experiment presented in Section 4.5.3, the background pressure in the vacuum chamber was $7 \cdot 10^{-3}$ mbar.

\subsubsection{XUV Diagnostics}

For spectrally resolved measurements we use a McPherson grazing incidence monochromator, model 248/310G. Several XUV diffraction gratings are available, of which we used the one with 133.6 grooves/mm in the experiments presented in this section. The XUV detection can be carried out by scanning a solar blind channel electron multiplier (channeltron) along the Rowland circle of the monochromator. We use a McPherson model 425 channeltron. At the time of writing this thesis, detection with a multichannel plate (MCP), model XUV-2040, which is also sensitive to IR radiation, was unsuccessful due to saturation by residual IR. For an absolute XUV power measurement we mounted an XUV photodiode (IRD, model AXUV 100 AL3), shielded against IR radiation with $150 \mathrm{~nm}$ and $300 \mathrm{~nm}$ thick Al filters. However, both filters were destroyed by the residual IR radiation, superimposed to the XUV beam. To solve this problem and enable both the MCP and the photodiode measurements, we plan to use additional Brewster plates, or, for a better XUV reflectivity, grazing-incidence optics with an anti-reflection coating for the IR (like the plate presented in [108]) for splitting the residual IR from the output coupled XUV.

\footnotetext{
${ }^{3}$ This picture was taken by Thorsten Näser.

${ }^{4}$ This was also confirmed by 109,110 .
} 


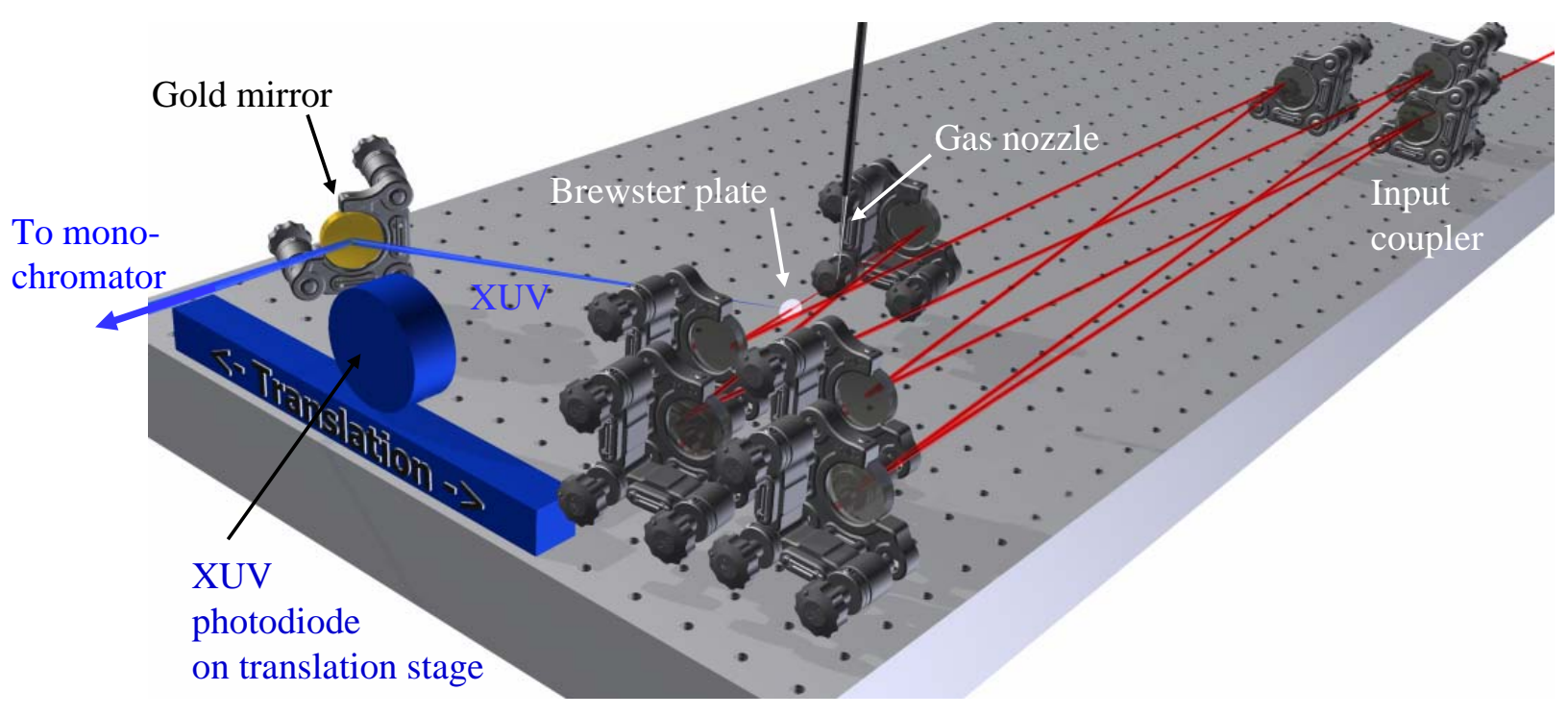

Figure 4.15: HHG generation and detection setup, see text for details. A close-up of the region around the focus is shown in Fig. 4.11. a.

Figure 4.15 shows a schematic of the XUV generation and detection setup, which was designed to offer maximum flexibility. The XUV beam coupled out by the Brewster plate is steered by a gold mirror to the entrance slit of the monochromator. Figure 4.16. a shows the power attenuation encountered by the XUV beam upon reflection at the Brewster plate and at the Au mirror as well as after having propagated through the residual Xe along the distance of $65 \mathrm{~cm}$ from the output coupler to the spectrometer. The reflectivity values were calculated for an angle of incidence of $56^{\circ}$, corresponding to Brewster's angle for fused silica with a refractive index of 1.45. The three combined power attenuation contributions are plotted in Figure 4.16. b. To enable a convenient switch between a spectrally resolved measurement and an integrated power measurement, we placed the XUV photodiode on a motorized translation stage, so that it can be readily driven in and out of the beam. This setup can easily be adapted to suit the related output coupling methods of the WOMOC and the GIP.

\subsubsection{XUV Generation - First Results}

\section{Locked Cavity}

In order to lie below the damage threshold of the free-standing $0.1 \mathrm{~mm}$ fused silica Brewster plate, we seeded the cavity with an average power of $7 \mathrm{~W}$. With an input coupler with $R=99.74 \%$ and 7 highly reflective mirrors, we measured a circulating average power level of $2.4 \mathrm{~kW}$ at the beginning of the experiment. The measured effective power enhancement was 340 with an intracavity pulse autocorrelation identical to the one of the input pulse.

\footnotetext{
${ }^{5}$ The graphic illustration was done by Jan Kaster.
} 


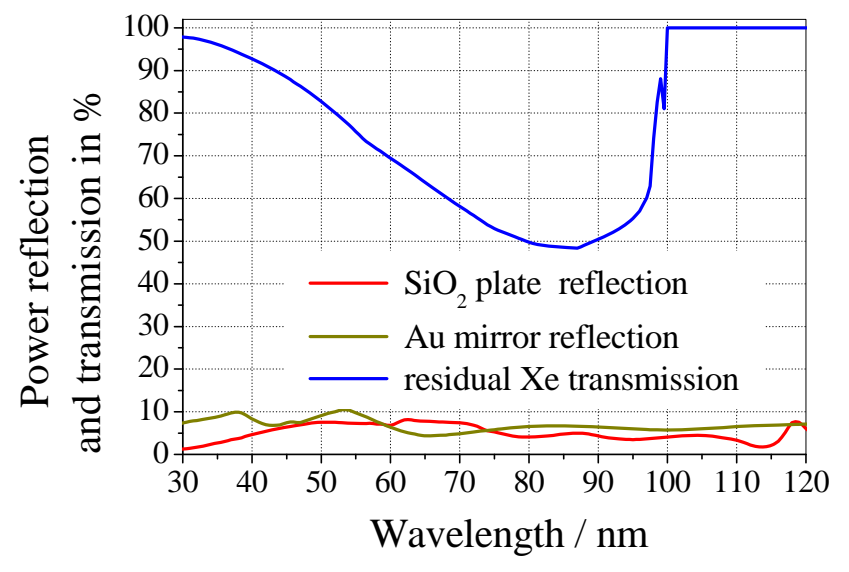

(a)

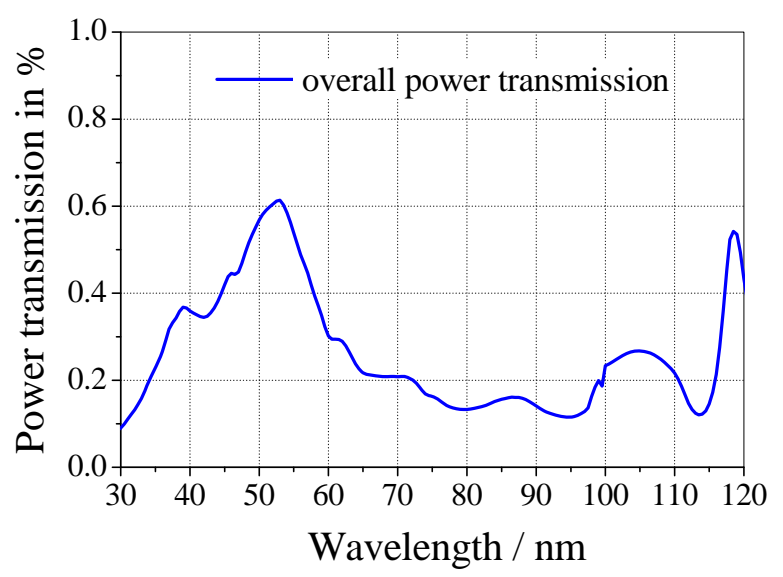

(b)

Figure 4.16: XUV power transmission from the focus to the spectrometer entrance: (a) separate contributions of the fused silica Brewster plate, of the gold steering mirror (both calculated with refractive index data from [112], for p polarization) and of the propagation along $65 \mathrm{~cm}$ of free space at a surrounding Xe pressure of $7 \cdot 10^{-3}$ mbar (data from [113]); (b) combined contributions.

We adjusted the cavity close to the center of the stability range, yielding a focus radius $w_{0} \approx 23 \mu \mathrm{m}$. This corresponds to an intensity of $2 \cdot 10^{13} \mathrm{~W} / \mathrm{cm}^{2}$ in the focus. The XUV yield was optimized by aligning the nozzle very close to the focus and by adjusting the backing pressure. The optimum for the latter was found to be approx. $1.2 \mathrm{bar}$. The measurement took several minutes, during which the intracavity power decreased to $1.8 \mathrm{~kW}$, corresponding to a power enhancement of 260 and an intensity of $1.5 \cdot 10^{13} \mathrm{~W} / \mathrm{cm}^{2}$ (the measurement was performed starting from long towards short wavelengths). Since the cavity without the Brewster plate can operate steadily for tens of minutes, the most likely cause of the circulating power decrease is the heating of the plate. Further experiments need to be performed in order to gain more insight into this process (see also Section 5.2). Figure 4.17.a shows the channeltron counts as the wavelength was scanned. Note that these are raw measurement data, i.e. no calibration of the detector was performed. Harmonics up to the $17^{\text {th }}$ order can be clearly observed. The roll-off of the noise level could be attributed to the slow power decrease discussed above. The broad width of the harmonics is caused by the relatively low resolution of the measurement (determined by the monochromator entrance slit size and the employed XUV diffraction grating). The cutoff frequency calculated with eq. (2.34) and with an average power of $2 \mathrm{~kW}$ (which is the value at the respective measurement point) corresponds to a wavelength of $70 \mathrm{~nm}$, see green dashed line in Fig. 4.17.a. The strongly decreased intensity of the $17^{\text {th }}$ harmonic indicates good agreement with the expected cutoff value. 


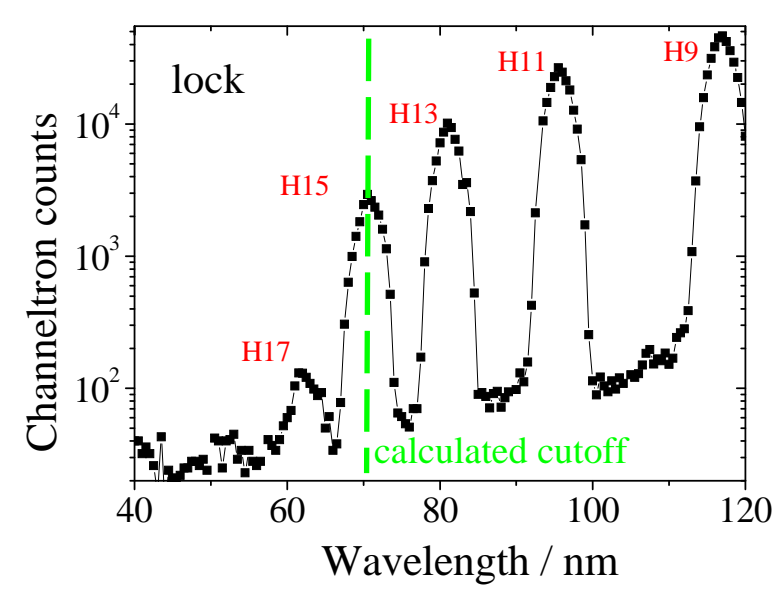

(a)

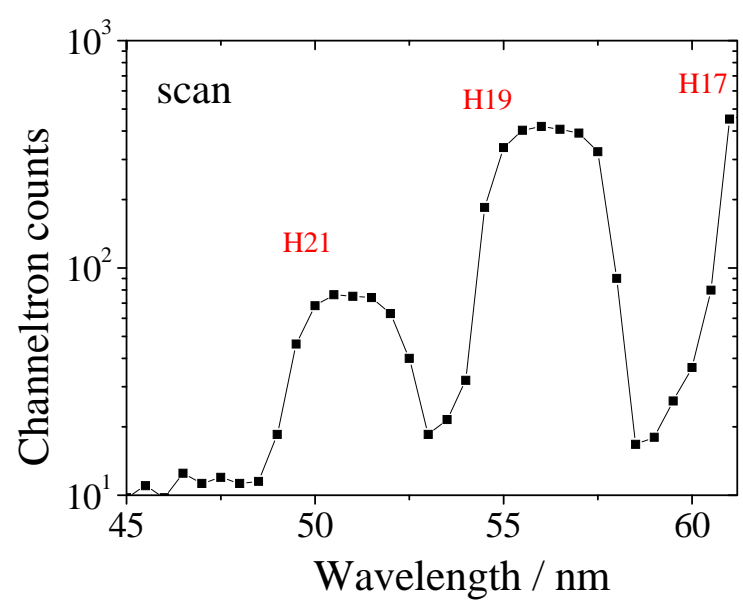

(b)

Figure 4.17: First XUV spectra recorded from the system presented in this thesis. (a) Locked state with $2.4 \mathrm{~kW}$ of circulating power (which decreased in time to $1.8 \mathrm{~kW}$ ), $200 \mathrm{fs}$ intracavity pulse duration. Green dashed line: calculated cutoff. See text for further details. (b) Spectrum recorded while scanning through the main resonance with $50 \mathrm{~W}$ of input power.

\section{Scan Mode}

To detect even higher harmonics without damaging the Brewster plate, we scanned the frequency comb through the matched cavity resonance periodically with an input power of $50 \mathrm{~W}$. In this way high intensities build up, but a large thermal load is avoided since the cavity is off-resonance most of the time. Figure 4.17.b shows the results for a $20 \mathrm{~Hz}$ scan over approximately half of the cavity FSR around the resonance (and with an increased channeltron counts integration time). In this regime, XUV up to the $21^{\text {st }}$ harmonic could be detected. While this mode of operation of the cavity does not serve the purpose of most envisaged experiments, it reveals that the achievable high intracavity power levels bear the potential of generating higher harmonics and more power per harmonic. This enforces the justification to search for better suited output couplers, which was done in the course of this thesis. 


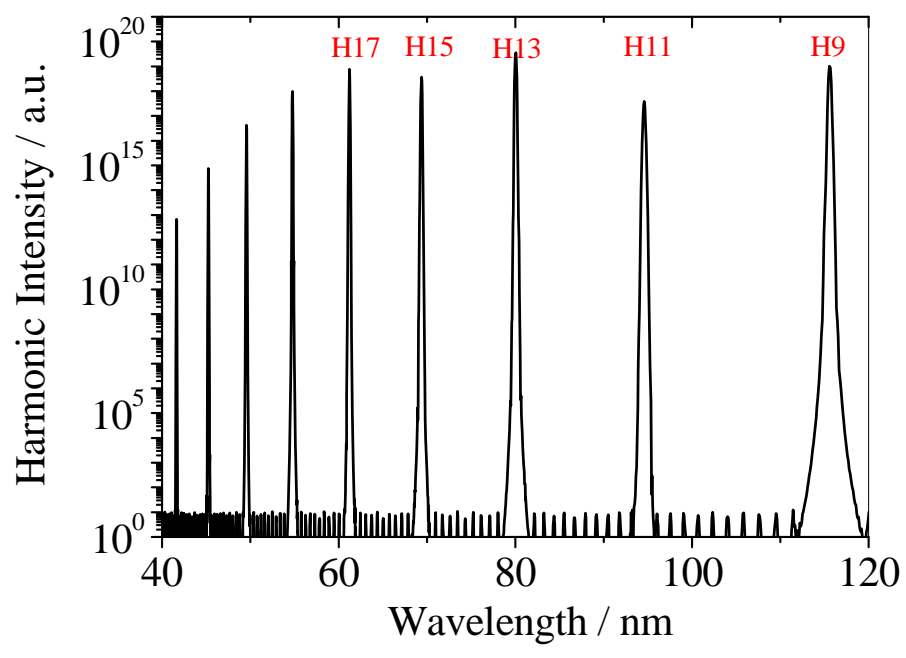

Figure 4.18: XUV spectrum calculated with the HHG model developed by Lewenstein et al. 77], see text for further details.

\section{Lewenstein Model Simulation}

For a further theoretical confirmation of this measurement, the harmonic spectrum was simulated [114] according to the Hydrogen-like atom model developed by Lewenstein et al., see Section III.D in [77]. The measured driving laser parameters from the beginning of the measurement (i.e. an intensity of $2 \cdot 10^{13} \mathrm{~W} / \mathrm{cm}^{2}$ ) were plugged in the simulation and perfect phase matching was assumed. The latter assumption is justified by the loose focusing on the one hand and by the empirical optimization of the gas pressure on the other hand. Figure 4.18 shows the result of the simulation ${ }^{6}$. The cutoff frequency is at the $\sim 17^{\text {th }}$ harmonic. Despite this slight mismatch, which is due to the power decrease during our measurement, this result corroborates the agreement between HHG theory and our measurement.

\footnotetext{
${ }^{6}$ This simulation was carried out by Ernst Fill
} 


\section{Chapter 5}

\section{Outlook}

\subsection{Further Power Scaling of Enhancement Cavities}

The power scaling results presented in Section 4.3 have shown that the enhancement in a standard-design passive cavity, built with state-of-the art, commercially available ionbeam sputtered dielectric multilayer mirrors, is primarily limited by nonlinear effects in the mirrors. An approach to circumventing this limitation consists in increasing the laser spot size on the mirrors, thus decreasing the incident intensity and relaxing nonlinear effects in the mirrors. Moreover, a larger irradiated area decreases the thermal gradient which is beneficial for the high-average-power regime. In this section we first report on two large-mode-area (LMA) enhancement cavity designs, in close analogy to [115], developed in our group as a consequence of the power scaling results presented in this thesis. Then we address two laser sources which will be employed for the next power scaling experiments. Combining these laser sources with the next-generation LMA cavities offers on the one hand the potential of scaling up the average power of femtosecond laser pulse enhancement to the MW level and on the other hand provides the prospect of few-cycle IR pulse enhancement.

\subsubsection{Novel Cavity Designs}

We discuss three ways of increasing the laser spot sizes on the mirrors of ring resonators that can be implemented separately or combined. The first method relies on increasing the angle of incidence (AOI) on the mirrors. The laser spot size increases proportionally to the reciprocal cosine of the AOI leading to a decrease of the intensity by the same factor. This approach can be implemented in a ring resonator configuration with oblique incidence (OI) on the mirrors. The radiation can be focused by two consecutively arranged off-axis parabolic mirrors, as shown in Fig. 5.1. 口 $^{\top}$ (the AOI in this example are $60^{\circ}$ ). The second approach relies on detuning the resonator from the center of the stability range by means of curved mirror distance and AOI variation. In so doing, the irradiated areas on the mirrors can be increased and the sizes of intra-cavity beam waist radii can be decreased.

\footnotetext{
${ }^{1}$ The graphic illustration was done by Jan Kaster.
} 
(a)

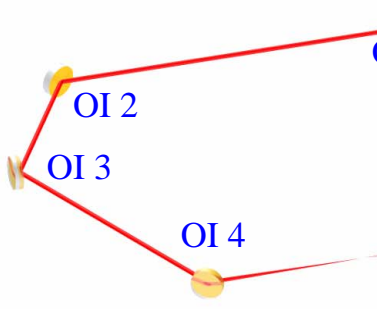

OI 1 / IC

(c)

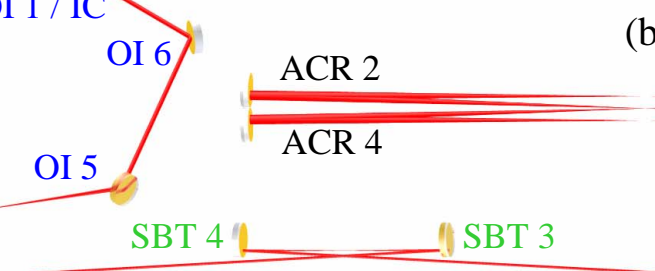

(b)

ACR 3

ACR 1 / IC

SBT 2

SBT 1 / IC

Figure 5.1: (a) Oblique incidence setup: OI 1 is the input coupling (IC) mirror, OI 4 and OI 5 are identical high reflecting (HR) off-axis parabolas and OI 2-4 are flat HR mirrors. (b) All-curved-mirror resonator: ACR 1 is the IC, the other mirrors are HR. All mirrors are curved with the same radius. (c) Standard bow tie setup: SBT 1 is a flat IC, SBT 2 is a flat HR mirror and SBT 3 and SBT 4 are curved HR mirrors with the same radius.
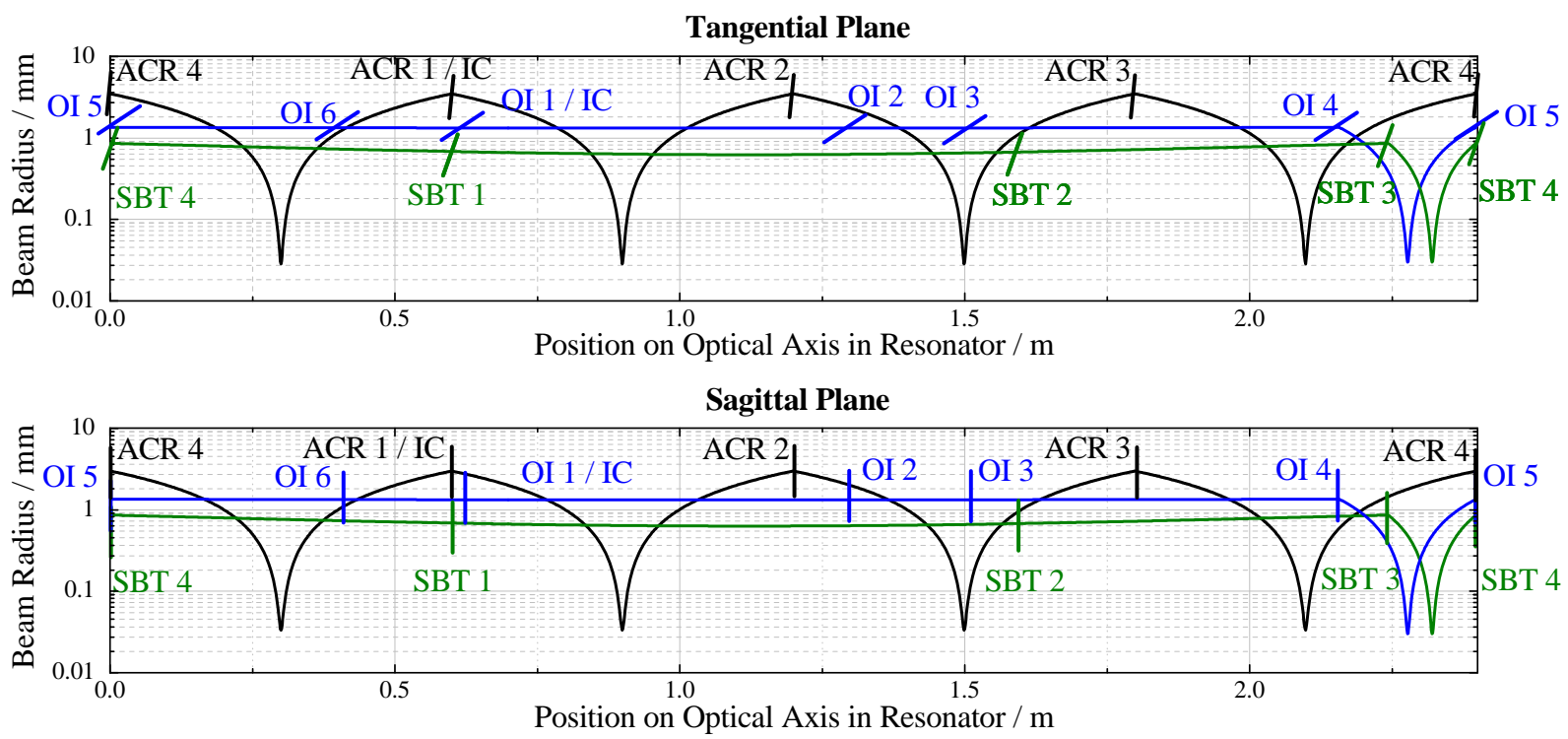

Figure 5.2: The $1 / e^{2}$-intensity radius along the optical axis of the three different cavity designs calculated with the ABCD matrix formalism for the tangential and sagittal planes, respectively. The mirror positions and AOI are indicated by markers drawn into the diagram. The laser spot sizes on the mirrors increase by the reciprocal cosine of the AOI. In the OI design the plotted beam dimension in the tangential plane has to be multiplied by a factor of $1 / \cos 0.96 \approx 1.74$ for the plane and by $1 / \cos 1.221 \approx 2.92$ for the parabolic mirrors. In the other two designs, this factor is almost 1. 
While this can be carried out in any stable resonator, we exemplify this method with a symmetric all-curved-mirror resonator (ACR) design, where all mirrors have the same radius of curvature, see Fig. 5.1.b. The main advantage of the symmetry is the fact that all spots on the mirrors are identical so that no mirror is exposed to an intensity larger than any of the others. Further advantages of this resonator design include multiple foci (the central focus could be used for noncollinear HHG with two circulating pulses [106, 116, 117]), easy access to the foci for Thomson scattering experiments (see Section 5.4) and compactness. The resonator is detuned from the center of the stability range by means of the distances between the mirrors. In this way, the beam radius on the mirrors is increased and the focus size decreased. The limit is given by the beam ellipticity owing to the nonzero AOI on the mirrors, diffraction losses at the finite surfaces of the mirrors and mechanical stability. The third method relies on increasing the cavity length by an integer factor. The achieved spot size variation depends on the resonator design. In the case of the ACR, the spot areas on the mirrors will be increased by the square of the number of circulating pulses.

In the following, we compare the OI and the ACR designs to the widely used standard bow tie (SBT) design consisting of two spherically curved and at least two plane mirrors, as shown in Fig. 5.1.c. For this comparison, we assume a central wavelength of $1046 \mathrm{~nm}$, a pulse duration of $250 \mathrm{fs}$, a pulse repetition rate of $250 \mathrm{MHz}$, two intra-cavity circulating pulses and fundamental-mode propagation with a beam waist radius of $30 \mu \mathrm{m}$. The pulse parameters were chosen according to the target parameters of a new laser system which is currently being built to seed the next-generation enhancement cavity in our group, see Section 5.1.2. Fixed beam waist radii correspond to certain positions in the resonator stability ranges and vice versa.

Figure 5.2 shows the beam radius along the cavity optical axis calculated with the $\mathrm{ABCD}$ matrix formalism. For the interpretation of the results of this comparison, it is essential to note that among the designs, several cavity parameters are not equivalent, such as e.g. the focal lengths $f$ of the focusing mirror ${ }^{2}$. The spot sizes on the mirrors are increased by a factor of 7.1 in the OI cavity (both focusing mirrors with $f=120 \mathrm{~mm}$ ) and in the ACR cavity (all mirrors with $f=150 \mathrm{~mm}$ ) by a factor of 14.1 compared to the SBT cavity (both focusing mirrors with $f=75 \mathrm{~mm}$, as in the system presented in this thesis).

The next-generation LMA enhancement cavity system to be built in our group will have an ACR design. Currently this design is being experimentally implemented by Jan Kaster. The reasons for the choice of this design over the OI resonator include the larger achievable beam radii on the mirrors, simpler alignment and increased flexibility. Moreover, the currently available parabolic mirrors for an OI resonator exhibit significantly lower surface quality than the available spherical mirrors.

The experimental realization of an ACR means new challenges. On the one hand, in general large spot sizes imply an increased misalignment sensitivity (see e.g. [118]) and thus, require an increased mechanical stability. On the other hand, pushing the limitation related

\footnotetext{
${ }^{2}$ For the OI cavity, $f$ was chosen according to current manufacturing limitations for parabolic mirrors, for the SBT design $f$ was chosen according to the system presented in this thesis and for the ACR design a convenient $f$ was chosen.
} 
to nonlinear-effects will also allow for much higher average powers. Thus, absorption, scattering and thermal lensing in the cavity mirrors are expected to play an increasingly important role. In particular, thermal lensing increases the misalignment sensitivity (see e.g. [119]) and affects the transverse mode matching. As a measure towards solving these new problems and optimizing high-power operation, we plan to investigate the thermal conduction in the cavity mirrors theoretically and experimentally.

\subsubsection{Novel Laser Sources}

There are two directions in which further power scaling of the enhancement cavity technique is being pursued in our group. On the one hand, we aim at scaling up the average power of few-hundred-fs pulses. On the other hand, we pursue the reduction of the enhanced pulse duration. In the following we give a brief review of the experiments planned in these two directions, with emphasis on the laser sources.

\section{Higher Average Power from Novel MHz Amplifier Systems}

Among the available high-repetition-rate femtosecond laser systems, the most rapid growth of available average output power in the last few years has been achieved with Yb-based systems. Compared to the power available when this work was started, i.e. $\sim 50 \mathrm{~W}[98]$, the power available now is approximately one order of magnitude larger. The most prominent techniques allowing for this growth are fiber amplifiers [120] and the Innoslab concept $[121$. 122]. Initial power scaling experiments with the novel LMA cavity, currently being built, are planned with a modified version of the system presented in [120], currently under construction in the frame of a collaboration of our group with the fiber laser group at the IAP in Jena. The target parameters are $250 \mathrm{fs}$ pulse duration with an average power of $500 \mathrm{~W}$ at a repetition rate of $250 \mathrm{MHz}$. For peak-intensity-related damage thresholds of state-of-the-art mirrors identified with our current setup, the ACR cavity in conjunction with the new seeding laser system offers the prospect of peak intensities on the order of $10^{15} \mathrm{~W} / \mathrm{cm}^{2}$ at intra-cavity beam waist radii of $30 \mu \mathrm{m}$. The circulating pulse duration is expected to remain unaffected and the circulating average power on the order of $1 \mathrm{MW}$. This regime is expected to boost the generated XUV power.

Besides highly efficient HHG, such a device with relatively long pulse durations would be ideally suited for the generation of hard X-ray radiation via inverse Compton (or Thomson) scattering, cf. Section 5.4. Here, the required laser pulse duration is lower bounded by the duration of the relativistic electron bunch and lies in the range of 1 ps. 


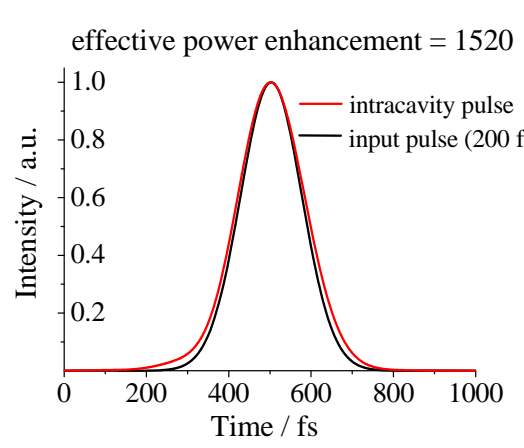

(a)

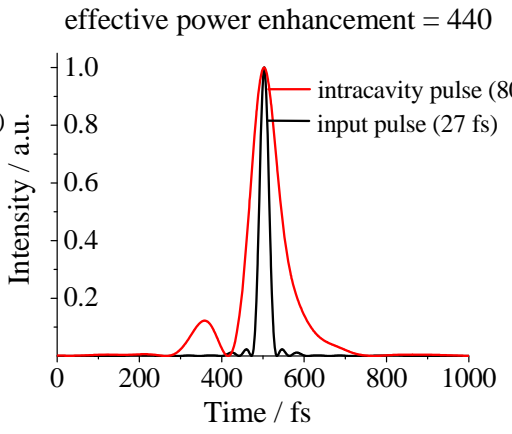

(b)

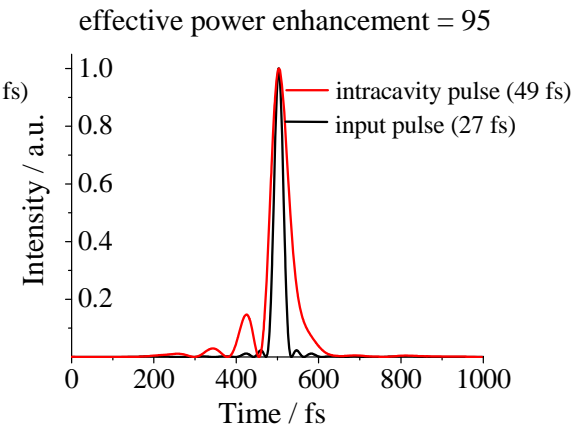

(c)

Figure 5.3: Simulated pulse enhancement for transform-limited input pulses. The curves are normalized for clarity. (a) The conditions of some of the experiments presented in this thesis: 200 fs input pulse, input coupler with $R=99.86 \%$ and 7 highly reflecting mirrors. (b) Same mirrors, but $27 \mathrm{fs}$ input pulse. The circulating pulse FWHM duration is $80 \mathrm{fs}$. (c) $27 \mathrm{fs}$ input pulse and decreased finesse (with same GDD as before). The circulating pulse FWHM duration decreases to 49 fs. These simulations were carried out by Simon Holzberger.

\section{Shorter Pulses Through Nonlinear Spectral Broadening}

To obtain few-cycle femtosecond pulses, Ti:Sa-based laser systems are customarily employed. However, the challenges imposed by the short pulse duration on Ti:Sa amplifiers $[123$ have been limiting the achieved average powers to values significantly lower than those of longer pulses generated with Yb-based systems. Nonlinear spectral broadening and subsequent temporal compression can be used to shorten the inherently multi-cycle high-power pulses of Yb-based systems. For instance, the original layout of the laser system employed in the experiments presented in this thesis contains a nonlinear compression stage (which is currently bypassed), with which a pulse duration as short as $27 \mathrm{fs}$ at an average output power exceeding $50 \mathrm{~W}$ was achieved at the repetition rate of $78 \mathrm{MHz}[98]$. In the meantime, even larger pulse energies have been obtained with the same technique, see e.g. [124], which were successfully used for direct, single-pass HHG. However, the process of spectral broadening in a fiber has both average and peak power limitations, which, in general, result in a longer pulse duration if the input parameters of the process are scaled up (e.g. $35 \mathrm{fs}$ in [124]). Therefore, a very promising approach to highly efficient HHG consists of strong single-pass nonlinear compression with relaxed conditions on the input power and subsequent passive enhancement of the compressed pulses.

In our group Simon Holzberger works on the activation of the nonlinear pulse compression stage of the seeding system (see [98]) and its inclusion into the existing experimental setup. This implies additional major challenges. Firstly, the effect of the cavity singleround-trip dispersion on the circulating pulse in the steady state is magnified by a factor on the order of the power enhancement [34]. Moreover, dispersion also leads to a spectral 
filtering of the seeding comb. Thus, a bandwidth vs. power enhancement trade-off needs to be found (cf. Section 2.1.2). To illustrate this, we have simulated the enhancement of the $200 \mathrm{fs}$ pulses obtained before the nonlinear compression stage as well as the enhancement of the spectrally broadened and compressed pulses in the current cavity, using the calculated reflectivity and GDD curves for the mirrors provided by the manufacturer. Figure 5.3. a depicts the enhancement of the $200 \mathrm{fs}$ pulses. The calculated effective power enhancement (i.e. circulating average power divided by input average power) of 1520 is in excellent agreement with the experimental results (see Chapter 4). The circulating pulse duration is not affected significantly. Figure 5.3. b shows the result of using the same mirrors and seeding the cavity with the measured spectrum of the compressed $27-\mathrm{fs}_{\mathrm{s}}$ pulses if a flat spectral phase is assumed. We observe an effective power enhancement decrease to 440 and an intracavity pulse duration increase to $80 \mathrm{fs}$. Calculating with a simulated set of mirrors having the same dispersion properties but lower reflectivity (input coupler $R=99.5 \%$ and 7 cavity mirrors with $R=(99.5 \%)^{(1 / 7)}$, i.e. providing an impedance matched peak enhancement of 200), yields the result depicted in Fig. 5.3.c. Due to the reduced finesse and better impedance matching, a broader spectrum is coupled to the cavity, leading to a shorter intracavity pulse duration of $49 \mathrm{fs}$. However, the effective power enhancement is also reduced to 95 . In conclusion, customized mirror designs with low GDD and/or the use of pairs of mirrors with opposite $G D D$ are necessary. Research on an optimum mirror design is currently being performed in our group in collaboration with Vladimir Pervak and coworkers.

Amplitude and phase fluctuations stemming from the nonlinear spectral broadening process might hamper the resonant enhancement. To the best of our knowledge, spectrally broadened pulses have not yet been enhanced in a passive resonator. Additional stabilization precautions might become necessary, including the detection and stabilization of the second laser parameter, as suggested by the 50-nm cases in Fig. 2.9 in Section 2.1.3.

\subsection{Power Scalable XUV Output Coupling}

The first XUV generation experiments carried out with the system presented in this work are in good agreement with the theoretical predictions, see Section 4.5. The current limitation for experimentally investigating the HHG process in the new power regimes available in femtosecond enhancement cavities is given by the output coupling mechanism. In order to access the XUV radiation generated in these power regimes, novel XUV output coupling techniques need to be implemented first. In the course of this work, several new output couplers were developed and proposed, however, not yet tested. In the following, the techniques coming into consideration for the systems developed in our group will be discussed. 


\subsubsection{Thin Plates and WOMOC}

A conceivable way to increase the damage threshold of thin plates, operated in transmission for the fundamental radiation and reflecting the XUV (such as Brewster plates or the GIP [108|), is improving their mounting with respect to cooling. In contrast to free-standing plates (see Fig. 4.11), the WOMOC is expected to allow a better cooling due to the fact that one surface of the wedged top layer, acting as the thin plate, is entirely attached to the underlying multilayer structure. To optimize cooling, the quantitative investigation of the heating effects is planned in our group. On the one hand, the in-situ measurement of the heating of these elements (and of the cavity mirrors) using a high-resolution thermal camera is planned. On the other hand, as for the cavity mirrors, we plan to develop a theoretical model for this process by numerically solving the heat conduction equation with appropriate boundary conditions.

\subsubsection{Mirrors with Apertures, Direct Output Coupling}

As mentioned in Section 4.4.3, mirrors with a circular hole with a suitable diameter have only very recently become available. Thus, to this day we have not carried out experiments with these novel mirrors. We expect that for a successful implementation of this method, additional precautions concerning the excited mode need to be taken. For instance, a position in the stability range will need to be chosen at which higher-order modes are not simultaneously resonant with the fundamental mode, thus avoiding a coupling among transverse modes. Additional spatial filtering with appropriate apertures might be necessary. Furthermore, the effect of the high intensity at the fundamental mode center on the hole edges is subject to future investigations. However, the major advantage of using the fundamental cavity mode is the already existing understanding of the HHG process for this transverse field distribution.

In parallel, quasi-imaging (QI) as presented in [37] is being investigated. To this end, curved mirrors with horizontal slits have been produced at the ILT in Aachen [107]. The manufacturing constraints of such slits are somewhat more relaxed than those of small holes, since the QI mode avoids the slit. In our cavity, the QI mode consisting of the degenerate Gauss-Hermite eigen-modes $G H_{00}$ and $G H_{40}$ experiences low losses even at slits with $\sim 300 \mu \mathrm{m}$ width. Initial power scaling results with such a mirror have revealed thermal limitations at around $5 \mathrm{~kW}$ of circulating power. The origin of this limitation is subject to further investigations. The production of new slotted mirrors with improved slit edges is planned in collaboration with the ILT. With those, further power scaling experiments and the investigation of thermal effects are planned. Moreover, in contrast to the fundamental mode, QI modes change their shape upon propagation along the optical axis due to the different Gouy phases of the involved resonator eigen-modes, cf. [37]. The relative rapid shape change in the region with an on-axis intensity maximum might affect the phasematching of the HHG process. Furthermore, the spatial distribution of the generated high harmonics still needs to be investigated and the question whether the generated XUV light will pass through the slit needs to be answered. 
Two further variations of the above methods are conceivable. Firstly, realizing QI in both transverse directions simultaneously could be achieved by canceling out the difference between the Gouy parameters for the two transverse directions, i.e. setting $\psi_{x}=\psi_{y}$. This could be done by horizontally tilting the beam incident on one of the curved mirrors in the current setup or by inserting an additional convex mirror [125]. In this case, a circular hole could be used instead of the slit. The rotational symmetric mode, which is a linear combination of Gauss-Laguerre eigen-modes in this case, might be beneficial for the HHG process. A second approach concerns the ACR design. To obtain relatively small foci, this resonator needs to be operated close to a stability range border. Therefore, QI in the center of the stability range cannot be achieved in this LMA cavity. However, as the stability range border is approached, the Gouy phase differences among higher-order modes decrease and the coupling among them increases. A quantitative description of this coupling is subject to further study ${ }^{3}$. We plan to investigate whether this coupling could be used to excite a QI-like mode in the ACR resonator with losses small enough so that they allow the desired power enhancement.

Finally, we mention that the currently implemented XUV detection setup (see Fig. 4.15) can easily be modified to suit the implementation of collinear, direct output coupling through a hole. The geometrically output coupled XUV beam can be steered to the XUV spectrometer by two parallel Au mirrors (or plates, anti-reflection coated for the IR [108]) under (close-to-) grazing incidence while the XUV photodiode mounted on the translation stage can remain at the same position.

\subsection{Towards Isolated as-Pulses Using fs-Enhancement Cavities}

In pump-probe experiments, probing with single pulses rather than a pulse train provides the best time resolution because the moment (or period) of observation is well-defined. In the frame of attosecond physics, the availability of isolated attosecond (as-) pulses at $\mathrm{MHz}$ repetition rates would enable new insights in the dynamics of the microcosm and is therefore highly desirable.

XUV as-pulses are generated with every linearly polarized optical half-cycle of the fundamental radiation driving the HHG process. Therefore, to obtain one isolated as-burst per driving fs pulse, either a single as-burst has to be isolated from the harmonic radiation generated by the driving pulse (which is usually a train of as-pulses), or the HHG process has to be confined to a single half-cycle of the driving pulse. The operation of methods following the first approach (e.g. amplitude gating [126]) is inherently limited to few-cycle pulses. Enhancing pulses this short in a passive resonator imposes extreme challenges to the mirror design. While the construction of such a setup would be a sensation, the second

\footnotetext{
${ }^{3}$ Fig. 8.a in the paper included [37], see Chapter 6, shows the power enhancement vs. detuning from the stability range center. The width of this curve is given by the coupling among transverse modes, which increases again as the position in the stability range approaches an instability boundary.
} 
approach seems more realistic for obtaining isolated attosecond pulses from fs-enhancement cavities in the near future. In the following we give a brief overview of the most prominent techniques in line with this approach and discuss aspects relevant for implementing HHG gating in an enhancement cavity.

Polarization gating $(\mathrm{PG})$ uses the fact that the HHG process is strongly polarizationdependent (see [127] and references therein). By combining two delayed counter-rotating circularly polarized pulses (with slightly different wavelengths), the polarization of the driving pulse is modulated in such a way that close-to linear polarization, necessary for HHG, is only achieved over a short time window within the resulting pulse, on the order of a half-cycle. The upper limit of the driving pulse duration is set by the ground state population depletion: if the pulse is too long, then the atoms will be fully ionized by the leading edge before the linear polarization gate starts and the as-burst can be generated. Two-color gating (TCG) employs waveforms synthesized from a fundamental-radiation pulse and its second harmonic ( $\mathrm{SH}$ ), which can increase the period between the generation of as-bursts to a full optical cycle of the fundamental, see e.g. [128. Another technique relying on the few-cycle duration of the driving pulses is ionization gating (see e.g. [129] and references therein). Here, the first cycle reaching the intensity required for HHG generates an as-pulse and the subsequent cycles fully ionize the gas atoms so that HHG cannot occur anymore. A powerful method working also with multi-cycle driving pulses is double optical gating (DOG) [130], which combines PG and TCG. Finally, generalized double optical gating (GDOG) [131, 132] employs two elliptically (instead of circularly) polarized pulses to reduce the ground state population depletion at the leading edge of the pulse. GDOG allows the generation of isolated as-bursts with fundamental radiation pulses as long as $28 \mathrm{fs}$, generated by a Ti:Sa laser system. This value already lies in the pulse duration range for which enhancement in a passive cavity has been demonstrated [17]. Due to the larger wavelength, for an Yb-based system the same number of optical cycles corresponds to a pulse duration of approximately $36 \mathrm{fs}$, which can be achieved with nonlinear compression, cf. Section 5.1.2. Therefore, from the point of view of the pulse duration, the prospect of intracavity gating is given.

Another encouraging result is provided by a test we performed in our cavity. To verify the resonance of the cavity for elliptically polarized seeding light, we placed a quarter-wave plate in the input beam, just in front of the cavity input coupler. While we rotated the plate over $360^{\circ}$, the cavity scan pattern did not change observably, implying that the cavity losses for any ellipticity of the input polarization are comparable. Therefore, we assume that any linear combination of elliptically polarized pulses can be resonant in the cavity. This is a prerequisite for the majority of the gating methods mentioned here. The unique power regime achievable in enhancement cavities, which provides multi-cycle pulses with large intensities at $\mathrm{MHz}$ repetition rates, might also open the door to novel combinations of the existing techniques (e.g. a combination of PG with ionization gating seems promising) or to completely new gating mechanisms.

It should also be mentioned that superimposing a SH portion to the fundamental driving the HHG process can be readily implemented in a single-pass fashion. Standard highly reflecting dielectric mirrors usually have a transmission band at the second harmonic fre- 
quency of the fundamental light. Thus, the mirror just before the HHG focus could serve as the input coupler for the fundamental radiation, while a SH portion is transmitted through this mirror and overlaps coherently with the intracavity circulating pulse in the HHG focus (but is not resonant in the cavity).

Recently, Durach et al. 133, 134 predicted a plasmonic metallization of thin dielectric films, illuminated by intense single-cycle laser pulses. Such a film could be used as a reflective surface onto which the intracavity generated as-pulse train impinges. If a second pulse, with a slightly different wavelength (e.g. a Ti:Sa-generated) impinges on this surface, the metallization follows the electric field of the pulse virtually instantaneously and thus, could switch the reflectivity of the surface. This phenomenon could in principle be used as an alternative to the above methods for isolating an as-pulse from a train.

\subsection{Other Experiments with High-Power Enhancement Cavities}

An alternative way for converting the laser light to short wavelength radiation involves scattering the laser pulse from a relativistic electron pulse [135. The relativistic Doppler shift leads to an increase of the photon energy by a factor $4 \gamma^{2}$, where $\gamma$ is the relativistic mass factor of the electrons. A $50 \mathrm{MeV}$ electron beam thus generates $50 \mathrm{keV}$ X-rays from $1.2 \mathrm{eV}$ photons. Due to the small Thomson cross-section, a high laser pulse energy and high charge of the electron bunch are required for generating a useful number of X-ray photons. The weak nature of the interaction may be considered an advantage in case of an enhancement cavity since it induces negligible depletion of the circulating laser pulses [136]. We point out that the ACR cavity design shown in Fig. 5.1.b is particularly suitable for Thomson scattering applications. Its geometry allows straightforward insertion of the electron pulse and extraction of the X-rays generated. If the electrons collide at a small angle with the laser pulse, only a small downshift of the X-ray photon energy is induced.

The high circulating power in the cavity combined with the high repetition rate affords applications of enhancement cavities in quite different fields of research. We mention multiphoton entanglement experiments [137], cavity-enhanced scattering [138], ultrasensitive absorption and dispersion spectroscopy [139], precision spectroscopy with helium [140], the generation of quantum frequency combs [141] and $\mathrm{THz}$ radiation generation [15]. For a successful realization of these applications a high circulating power is of crucial importance. 


\section{Chapter 6}

\section{Included Publications}

This chapter contains the journal papers included in this cumulative thesis. First, an overview of the publications and the thesis author's contribution to the results presented therein is given. Subsequently, the papers are included in their original form 1 .

[33] I. Pupeza, T. Eidam, J. Rauschenberger, B. Bernhardt, A. Ozawa, E. Fill, A. Apolonski, Th. Udem, J. Limpert, Z. A. Alahmed, A. M. Azzeer, A. Tünnermann, T. W. Hänsch and F. Krausz, "Power scaling of a high repetition rate enhancement cavity," Opt. Letters 12, 2052-2054 (2010). http://www.opticsinfobase.org/abstract.cfm?URI=ol-35-12-2052

In this paper we present our enhancement cavity for the first time. The largest circulating pulse energy in a high-repetition rate femtosecond laser resonator to date is demonstrated. Power scaling limitations are investigated. The major part of the novel results presented in this paper can be attributed to the author of this thesis.

[34] I. Pupeza, X. Gu, E. Fill, T. Eidam, J. Limpert, A. Tünnermann, F. Krausz, Th. Udem, "Highly sensitive dispersion measurement of a high-power passive optical resonator using spatial-spectral interferometry," Opt. Express 18, 2618-26295 (2010). http://www.opticsinfobase. org/abstract.cfm?URI=oe-18-25-26184

In this paper, the first highly sensitive intracavity dispersion measurement method working at high intra-cavity intensities was developed, based on spatially and spectrally resolved interferometry (SSI) of a copy of the input beam to the cavity with a copy of the circulating beam. While most of the experimental work and data interpretation were done by the author of this thesis, the initial idea of applying SSI as well as great support with the

\footnotetext{
${ }^{1}$ These paper were published in Optics Letters and Optics Express and are made available as electronic reprints with the permission of OSA. The URL's at which the papers can be found on the OSA website are given in the citations. Systematic or multiple reproduction or distribution to multiple locations via electronic or other means is prohibited and is subject to penalties under law.
} 
experiment and data interpretation came from Xun $\mathrm{Gu}$, along with substantial support from the other authors.

[35] I. Pupeza, E. Fill, F. Krausz, "Low-loss VIS/IR-XUV beam splitter for high-power applications," Opt. Express 19, 12108-12118 (2011). http://www .opticsinfobase.org/abstract.cfm?URI=oe-19-13-12108

This paper deals with a novel optical element designed to spatially split a high-power laser beam from a collinearly superimposed beam consisting of its high harmonics. In particular, due to improved heat conduction, this element is expected to enable the power scalability of HHG enhancement cavities. The major part of the novel results presented in this paper can be attributed to the author of this thesis.

[36] Y.-Y. Yang, F. Süßmann, S. Zherebtsov, I. Pupeza, J. Kaster, D. Lehr, H.-J. Fuchs, E.-B. Kley, E. Fill, X.-M. Duan, Z.-S. Zhao, F. Krausz, S. L. Stebbings, M. F. Kling, "Optimization and characterization of a highly-efficient diffraction nanograting for MHz XUV pulses," Opt. Express 19, 1955-1962 (2011). http://www .opticsinfobase.org/abstract.cfm?URI=oe-19-3-1954

In this paper, the optical element initially presented by Yost et al. in [19 is optimized with respect to its XUV efficiency. The contribution of the author of this thesis lies mainly in setting up and performing the characterization of the optical element for IR radiation (Section 4). In particular, this contribution includes the modification of the lossmeter for operation with arbitrary angles of incidence and the z-scan setup, built and operated together with Jan Kaster. The nonlinearity enhancement of the optics owed to the nanostructure, which was measured in the z-scan setup, is a result relevant to this thesis in that it exhibits a potential disadvantage with respect to power scaling.

[37] J. Weitenberg, P. Rußbüldt, T. Eidam, I. Pupeza, "Transverse mode tailoring in a quasi-imaging high-finesse femtosecond enhancement cavity," Opt. Express 19, 9551-9561 (2011). http://www.opticsinfobase.org/abstract.cfm?URI=oe-19-10-9551

In this paper, the concept of quasi-imaging (QI) is introduced. QI uses a subset of degenerate resonant transverse modes in an optically stable resonator to excite a field distribution which simultaneously has an intensity maximum on the optical axis and avoids an onaxis obstacle far away from this maximum. This concept is particularly interesting as a potential means of coupling out harmonics generated in a high-intensity focus through a hole in a resonator mirror, which is avoided by the QI transverse field distribution. An important experimental result is the fact that in our 3000-finesse cavity, quasi-imaging can be achieved without any additional active control. The experiments were performed mainly by the author of this thesis who has also contributed to the theoretical part of the work. 


\title{
Power scaling of a high-repetition-rate enhancement cavity
}

\author{
Ioachim Pupeza, ${ }^{1,2, *}$ Tino Eidam, ${ }^{3}$ Jens Rauschenberger, ${ }^{1,2}$ Birgitta Bernhardt, ${ }^{1}$ Akira Ozawa, ${ }^{1}$ Ernst Fill, ${ }^{1}$ \\ Alexander Apolonski, ${ }^{2}$ Thomas Udem, ${ }^{1}$ Jens Limpert, ${ }^{3}$ Zeyad A. Alahmed, ${ }^{4}$ Abdallah M. Azzeer, ${ }^{4}$ \\ Andreas Tünnermann, ${ }^{3}$ Theodor W. Hänsch, ${ }^{1,2}$ and Ferenc Krausz ${ }^{1,2}$ \\ ${ }^{1}$ Max-Planck-Institut für Quantenoptik, Hans-Kopfermann-Strasse 1, 85748 Garching, Germany \\ ${ }^{2}$ Ludwig-Maximilians-Universität München, Department für Physik, Am Coulombwall 1, 85748 Garching, Germany \\ ${ }^{3}$ Friedrich-Schiller-Universität Jena, Institut für Angewandte Physik, Albert-Einstein-Strasse 15, 07745 Jena, Germany \\ ${ }^{4}$ King Saud University, Department of Physics and Astronomy, P.O. Box 2455, 11451 Riyadh, Saudi Arabia \\ ${ }^{*}$ Corresponding author: ioachim.pupeza@mpq.mpg.de
}

Received January 28, 2010; revised April 20, 2010; accepted May 26, 2010; posted June 1, 2010 (Doc. ID 123499); published June 9, 2010

\begin{abstract}
A passive optical resonator is used to enhance the power of a pulsed $78 \mathrm{MHz}$ repetition rate Yb laser providing $200 \mathrm{fs}$ pulses. We find limitations relating to the achievable time-averaged and peak power, which we distinguish by varying the duration of the input pulses. An intracavity average power of $18 \mathrm{~kW}$ is generated with close to Fourier-limited pulses of $10 \mathrm{~W}$ average power. Beyond this power level, intensity-related effects lead to resonator instabilities, which can be removed by chirping the seed laser pulses. By extending the pulse duration in this way to 2 ps, we could obtain $72 \mathrm{~kW}$ of intracavity circulating power with $50 \mathrm{~W}$ of input power. (c) 2010 Optical Society of America OCIS codes: $320.7090,060.2320,320.7160,140.4780,190.4160$.
\end{abstract}

Efforts to resonantly enhance pulsed lasers in an external cavity have recently been boosted by the prospect of intracavity high-harmonic generation (HHG) [1-5]. Traditional methods of generating peak intensities exceeding $10^{13} \mathrm{~W} / \mathrm{cm}^{2}$ required for HHG rely on a largely reduced pulse repetition rate (see, e.g., [6]). The intracavity approach allows HHG with a multimegahertz repetition rate such that individual modes of the resulting frequency comb [7] in the extreme UV (XUV) may be used as cw lasers. Such a laser source would be highly desirable for high-resolution spectroscopy in this hitherto inaccessible wavelength region. Further applications of such a compact and coherent XUV source include lithography or XUV optics characterization. Yet another emerging application, for which compact enhancement cavities constitute a very promising approach, is the generation of highbrilliance hard $\mathrm{x}$-rays via inverse Compton scattering of laser photons by a relativistic electron beam, Doppler upshifting them to the hard x-ray or even gamma-ray range $[8,9]$.

Enhancing a train of pulses in an optical resonator is analogous to the cw case if the mode spacing of the pulse train matches the resonator's frequency-resolved free spectral range. In the time domain this means that, after each round trip, the pulse circulating in the passive cavity interferes constructively with the next pulse from the laser. A power enhancement of a few thousands has been achieved so far with enhancement cavities seeded by Ti: sapphire [1-3] and Yb-doped [4,5] lasers reaching a few kilowatts of intracavity average power and peak intensities exceeding $10^{13} \mathrm{~W} / \mathrm{cm}^{2}$ at the cavity focus. With the advent of high-power ultrafast laser systems [10-12], the power scalability investigation of enhancement cavities becomes crucial to the development of this technique. In this Letter, we explore limiting factors for the intracavity power in a high-repetition-rate, bow-tie cavity employing state-of-the-art commercially available ion-beam sputtered dielectric mirrors (Layertec).
We seed our external cavity with the system presented in [10] [see Fig. 1(a)]. Transform-limited $170 \mathrm{fs} \mathrm{sech}^{2}$ initial pulses are generated by a passively mode-locked, diode-pumped Yb:KYW oscillator with $78 \mathrm{MHz}$ repetition rate and $220 \mathrm{~mW}$ average power. The FWHM bandwidth
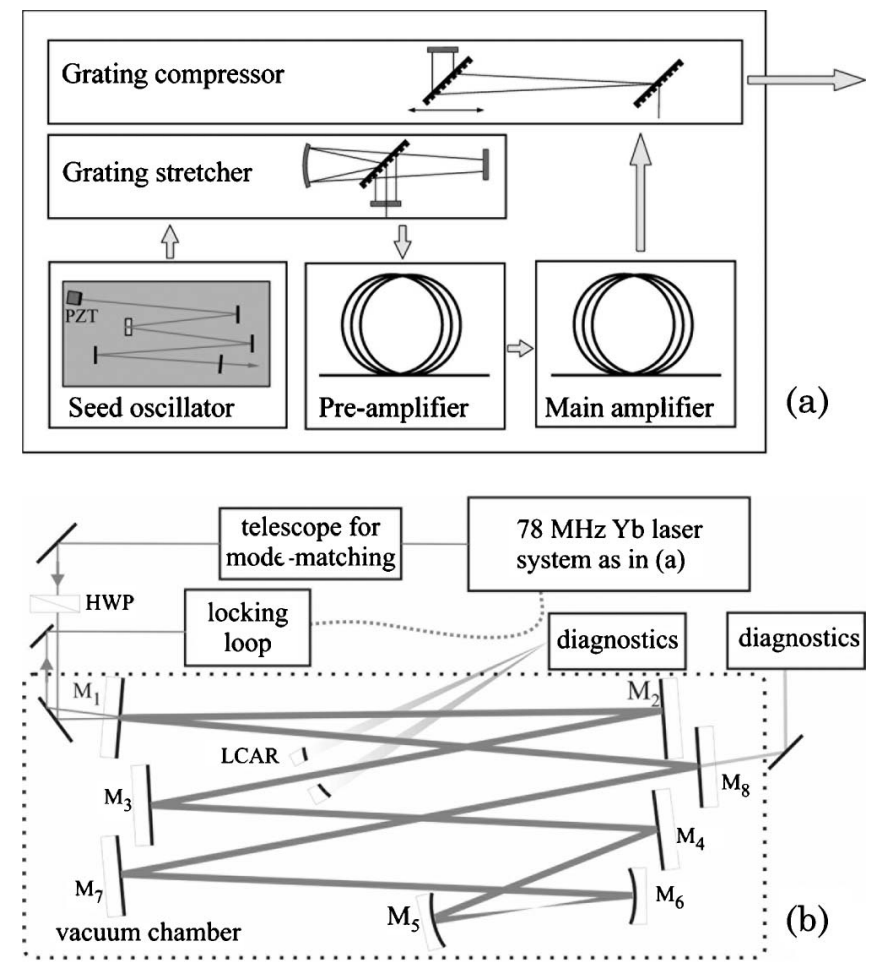

Fig. 1. Experimental setup: (a) seeding laser [10] and (b) enhancement cavity. M2-M8, dielectric mirrors with $R=99.995 \%$ $\pm 20 \mathrm{ppm}$ (parts per million) (ring-down measurement); M1, $99.86 \%$ reflectivity input coupler. M5 and M6 have a radius of curvature of $150 \mathrm{~mm}$ and enclose the $22 \mu \mathrm{m}$ cavity focus $\left(1 / e^{2}\right.$ radius calculated at the stability range center). HWP, half-wave plate; LCAR, large circular aperture reflector; diagnostics, photodiode/power meter/spectrometer/autocorrelator/beam profiler. 
is $7 \mathrm{~nm}$ centered around $1042 \mathrm{~nm}$. The pulses are stretched to $150 \mathrm{ps}$ with a transmission grating and sent to a two-stage fiber amplifier. The amplifier fibers are pumped at $976 \mathrm{~nm}$ by laser diodes delivering up to 25 and $130 \mathrm{~W}$. Subsequent compression down to $200 \mathrm{fs}$ is achieved with two fused-silica transmission gratings. By varying the distance between these gratings, the pulse length can be adjusted between $200 \mathrm{fs}$ and more than $10 \mathrm{ps}$ without affecting the other beam parameters. After compression, the average power exceeds $50 \mathrm{~W}$. The output spectrum is largely independent of the amplification level.

As in the work presented in [1-5], our enhancement cavity is a ring resonator whose round-trip time is adjusted to the inverse of the seeding laser repetition rate [see Fig. 1(b)]. For compactness, the beam is folded several times. The $1 / e^{2}$ beam diameters on the cavity mirrors range between 1.5 and $2.6 \mathrm{~mm}$. To minimize group delay dispersion (GDD) and losses, the cavity is placed inside a vacuum chamber. Its optics exhibit very low dispersion (GDD $<20 \mathrm{fs}^{2}$ per mirror, according to the manufacturer's specification) over the bandwidth of the $200 \mathrm{fs}$ seed pulses, making further dispersion compensation unnecessary. The frequency of the $n$th laser mode is given by $f_{n}=n f_{\text {rep }}+f_{\mathrm{CE}}$, where $f_{\text {rep }}$ is the laser repetition frequency and $f_{\mathrm{CE}}$ is the carrier-envelope (CE) frequency [1]. Because of the relatively narrow bandwidth of the seeding laser, small variations of $f_{\text {rep }}$ and $f_{\mathrm{CE}}$ have very similar effects on the structure of the frequency comb. Thus, a stable lock of the seeding laser to the enhancement cavity can be achieved by actively controlling a single comb parameter. In our case, this control is obtained by varying the position of an oscillator cavity end mirror with a fast piezoelectric transducer. The lock is realized with a Hänsch-Couillaud scheme [13]. In contrast to the original scheme, where an intracavity Brewster plate is employed, in our case the necessary polarization discrimination is given by the nonorthogonal incidence on the mirrors. In addition, for optimum enhancement, a coarse CE-offset adjustment is achieved by manually varying the seed oscillator optical pump power. The power enhancement factor $P$ is defined as $P=P_{\text {circ }} / P_{\text {in }}$, where $P_{\text {circ }}$ and $P_{\text {in }}$ denote the circulating intracavity power and the seeding laser power, respectively. We determine $P_{\text {circ }}$ by two different methods. We measure the power $P_{\text {leak }}$ leaking through a cavity mirror and divide it by the mirror transmission of $1.65 \times 10^{-6}$. To prevent measurement errors due to potential mirror transmission changes at higher powers, we implement a second measurement method for $P_{\text {circ }}$ using a large circular aperture around the intracavity laser beam [see Fig. 1(b)]. This aperture does not affect the enhancement but still reflects a measurable portion $P_{\text {refl }}$ of the intracavity light. Moreover, by using the leakage through the diagnostic mirror, we record the beam profile with a CCD camera and measure the intracavity autocorrelation and spectrum.

In a first experiment, we investigated the enhancement for various input powers $P_{\text {in }}$ while keeping the input pulse duration at $200 \mathrm{fs}$. For $35 \mathrm{~mW} \leq P_{\text {in }} \leq 10 \mathrm{~W}$, we observe a nearly linear dependence of both $P_{\text {leak }}$ and $P_{\text {refl }}$ on $P_{\text {in }}$ [see Fig. 2(a)] in a stable locking regime. The entire spectrum was coupled into the cavity and uniformly enhanced, with the intracavity autocorrelation remaining constant. The reflection from the input coupler indicated an input coupling ratio of $\sim 65 \%$. These results imply a nearly constant enhancement factor $P=1800$ and are in excellent agreement with the expected values assuming a lossless input coupler and perfect transversal mode matching. For $P_{\text {in }}=10 \mathrm{~W}$, a circulating power $P_{\text {circ }}=$ $18 \mathrm{~kW}$ was reached. As discussed later in the text, a cavity transverse mode variation was observed with increasing power. For input powers beyond $10 \mathrm{~W}$, the lock became increasingly unstable and, during the short periods of resonance, an intracavity pulse duration between 300 and $400 \mathrm{fs}$ was observed. Operation beyond $10 \mathrm{~W}$ of input power invariably led to mirror damage.

To further investigate the limits of enhancement, we conducted a second scaling experiment. To distinguish between purely thermal effects and effects involving nonlinear processes in the cavity mirrors, the circulating pulse duration $\tau_{\text {circ }}$ was varied by chirping the input pulses, while keeping $P_{\text {in }}=50 \mathrm{~W}$ constant. The results are shown in Fig. 2(b). For $\tau_{\text {circ }} \geq 2$ ps, the locking was stable with an intracavity circulating power of $P_{\text {circ }}=$ $72 \mathrm{~kW}$, which corresponds to an enhancement factor $P=1400$. The input coupling ratio to the cavity amounted to $\sim 50 \%$. As the pulse duration was decreased from 2 ps toward $640 \mathrm{fs}$, the lock became increasingly unstable and the enhancement factor decreased. For $\tau_{\text {circ }}<640$ fs, mirror damage occurred repeatedly. Damages were observed not only on the mirror with the minimum impinging beam size, where the peak intensity reached around $10^{11} \mathrm{~W} / \mathrm{cm}^{2}$, but occasionally, the mirror with the maximum beam size, implying a roughly 3 times lower peak intensity, was also damaged. This behavior is subject to further investigation. Thus, for $P_{\text {in }}=50 \mathrm{~W}$, the
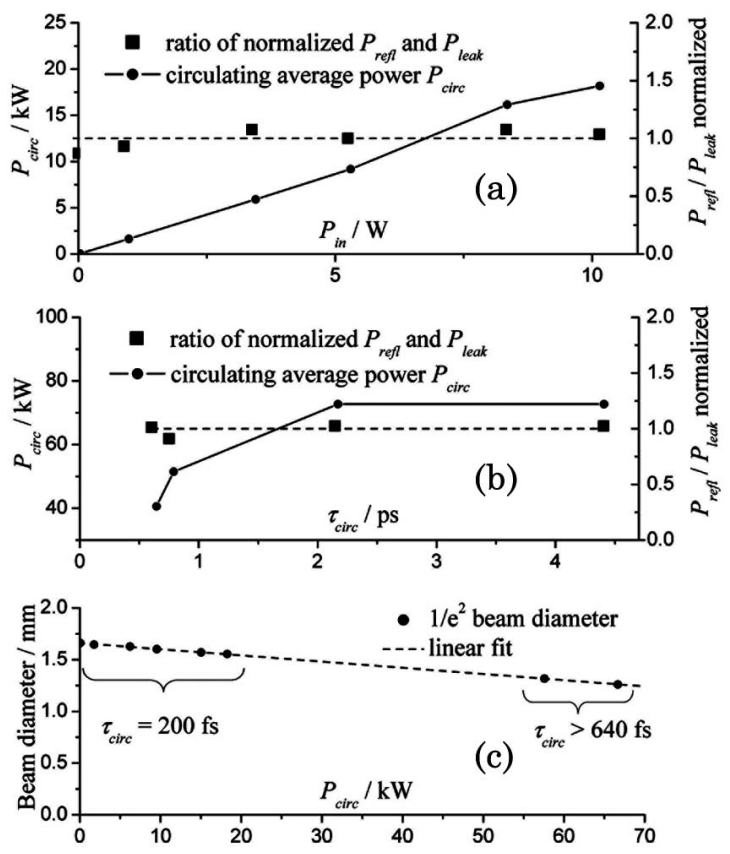

Fig. 2. (a) Circulating power versus input power for a constant intracavity pulse duration of $200 \mathrm{fs}$. (b) Circulating power versus pulse duration for constant input power of $50 \mathrm{~W}$. The squares show the ratio of the values of $P_{\text {refl }}$ and $P_{\text {leak }}$ normalized to their average. (c) Beam diameter $\left(1 / e^{2}\right.$ value, measured behind diagnostic mirror) versus $P_{\text {circ }}$ (for different pulse durations). 
decrease of both stability and the enhancement factor and mirror damage primarily relate to the peak power. The small variation of the normalized values of $P_{\text {leak }}$ and $P_{\text {refl }}$, shown in Fig. 2, implies that the transmission of the diagnostic mirror remained constant during all experiments.

Moreover, we observed a circulating power-dependent variation of the cavity transverse mode with a CCD camera placed behind the diagnostic mirror; see Fig. 2(c). We believe that this effect is due to thermal lensing in the cavity mirrors since it scales with the average circulating power, and is independent of pulse duration. However, the exact physical mechanisms for the change in beam diameter and for instabilities at higher peak powers still need to be investigated. The thermal nature of the beam diameter change is also supported by the observation of a delay of roughly $1 \mathrm{~s}$ from the moment the lock is initiated until the intracavity power settled to a steady state. We attribute the reduction of the cavity enhancement from 1800 at low circulating powers to 1400 at the maximum power to changes of the intracavity transverse mode profile due to this effect. This leads to a less than optimum overlap of the incoming and the circulating beam at the cavity input coupler. The decreasing input coupling ratio to the cavity with increasing $P_{\text {circ }}$ confirms this assumption.

In conclusion, we have investigated power scaling limitations of a $78 \mathrm{MHz}$ repetition rate, bow-tie enhancement cavity. By comparing the enhancement behavior for constant circulating power and varying the pulse duration, we found that high peak power is the primary cause of mirror damage. Below this damage threshold, in a stable locking regime, pulse duration can be traded in versus circulating power. A maximum circulating power of $18 \mathrm{~kW}$ was achieved for the minimum pulse duration of $200 \mathrm{fs}$, and a minimum pulse duration of 2 ps was measured for the maximum circulating power of $72 \mathrm{~kW}$. In the cavity focus, these peak powers lead to intensities exceeding $10^{14} \mathrm{~W} / \mathrm{cm}^{2}$, derived with the calculated focus diameter. With the demonstrated peak power, stronger focusing (e.g., as reported in [5]) would increase the peak intensity in the focus by another order of magnitude. This enhancement cavity offers the prospect of HHG as well as hard x-ray generation via inverse Compton scattering at previously unachieved power levels. Further increase of the supported intracavity peak power calls for advances in mirror technology as well as cavity design.

This work was supported by the Deutsche Forschungsgemeinschaft (DFG) Cluster of Excellence, Munich Centre for Advanced Photonics (MAP) (www.munichphotonics.de), by the KORONA Max-Planck-Institut für Quantenoptik (MPQ)/Fraunhofer Institut für Lasertechnik (ILT) cooperation and the King Saud University (KSU)/ MPQ collaboration.

\section{References}

1. C. Gohle, Th. Udem, M. Herrmann, J. Rauschenberger, R. Holzwarth, H. A. Schuessler, F. Krausz, and T. W. Hänsch, Nature 436, 234 (2005).

2. R. J. Jones, K. D. Moll, M. J. Thorpe, and J. Ye, Phys. Rev. Lett. 94, 193201 (2005).

3. A. Ozawa, J. Rauschenberger, C. Gohle, M. Herrmann, D. R. Walker, V. Pervak, A. Fernandez, R. Graf, A. Apolonski, R. Holzwarth, F. Krausz, T. W. Hänsch, and Th. Udem, Phys. Rev. Lett. 100, 253901 (2008).

4. D. C. Yost, T. R. Schibli, and J. Ye, Opt. Lett. 33, 1099 (2008).

5. I. Hartl, T. R. Schibli, A. Marcinkevicius, D. C. Yost, D. D. Hudson, M. E. Fermann, and J. Ye, Opt. Lett. 32, 2870 (2007).

6. M. Hentschel, Z. Cheng, F. Krausz, and Ch. Spielmann, Appl. Phys. B 70 [Suppl.], S161 (2000).

7. D. C. Yost, T. R. Schibli, J. Ye, J. L. Tate, J. Hostetter, M. B. Gaarde, and K. J. Schafer, Nature Phys. 5, 815 (2009).

8. F. X. Kaertner, W. S. Graves, D. E. Moncton, and F. O. Ilday, "Compact, high-flux, short-pulse x-ray source," U.S. patent application 20060251217 (November 9, 2006).

9. F. V. Hartemann, W. J. Brown, D. J. Gibson, S. G. Anderson, A. M. Tremaine, P. T. Springer, A. J. Wootton, E. P. Hartouni, and C. P. J. Barty, Phys. Rev. ST Accel. Beams 8, 100702 (2005).

10. T. Eidam, F. Röser, O. Schmidt, J. Limpert, and A. Tünnermann, Appl. Phys. B 92, 9 (2008).

11. P. Russbüldt, T. Mans, G. Rotarius, J. Weitenberg, H. D. Hoffmann, and R. Poprawe, Opt. Express 17, 12230 (2009).

12. T. Eidam, S. Hanf, E. Seise, T. V. Andersen, T. Gabler, C. Wirth, T. Schreiber, J. Limpert, and A. Tünnermann, Opt. Lett. 35, 94 (2010).

13. T. W. Hänsch and B. Couillaud, Opt. Commun. 35, 441 (1980). 


\title{
Highly sensitive dispersion measurement of a high-power passive optical resonator using spatial-spectral interferometry
}

\author{
Ioachim Pupeza ${ }^{1, *}$, Xun Gu$^{1}$, Ernst Fill ${ }^{1}$, Tino Eidam ${ }^{2}$, Jens Limpert ${ }^{2}$, \\ Andreas Tünnermann ${ }^{2}$, Ferenc Krausz ${ }^{1}$, and Thomas Udem ${ }^{1}$ \\ ${ }^{1}$ Max-Planck-Institut für Quantenoptik, \\ Hans-Kopfermann-Str. 1, 85748 Garching, Germany \\ ${ }^{2}$ Friedrich-Schiller-Universität Jena, Institut für Angewandte Physik, \\ Albert-Einstein-Str. 15, 07745 Jena, Germany \\ *ioachim.pupeza@mpq.mpg.de
}

\begin{abstract}
We apply spatially and spectrally resolved interferometry to measure the complex ratio between the field circulating inside a high-finesse femtosecond enhancement cavity and the seeding field. Our simple and highly sensitive method enables the measurement of single-round-trip group delay dispersion of a fully loaded cavity at resonance for the first time. Group delay dispersion can be determined with a reproducibility better than $1 \mathrm{fs}^{2}$ allowing the investigation of nonlinear processes triggered by the high intracavity power. The required data acquisition time is less than $1 \mathrm{~s}$.
\end{abstract}

(C) 2010 Optical Society of America

OCIS codes: (140.4050) Mode-locked lasers; (120.0120) Instrumentation, measurement, and metrology; (320.7100) Ultrafast measurements; (230.5750) Resonators.

\section{References and links}

1. G. Rempe, R. J. Thompson, H. J. Kimble, and R. Lalezari, "Measurement of ultralow losses in an optical interferometer," Opt. Lett. 17, 363-365 (1992).

2. C. Gohle, Th. Udem, M. Herrmann, J. Rauschenberger, R. Holzwarth, H. A. Schuessler, F. Krausz, and T. W. Hänsch, "A frequency comb in the extreme ultraviolet," Nature 436, 234-237 (2005).

3. R. J. Jones, K. D. Moll, M. J. Thorpe, and J. Ye, "Phase-Coherent Frequency Combs in the Vacuum Ultraviolet via High-Harmonic Generation inside a Femtosecond Enhancement Cavity," Phys. Rev. Lett. 94, 193201 (2005).

4. A. Ozawa, J. Rauschenberger, C. Gohle, M. Herrmann, D. R. Walker, V. Pervak, A. Fernandez, R. Graf, A. Apolonski, R. Holzwarth, F. Krausz, T. W. Hänsch, and Th. Udem, "High Harmonic Frequency Combs for High Resolution Spectroscopy," Phys. Rev. Lett. 100, 253901 (2008).

5. D. C. Yost, T. R. Schibli, and J. Ye, "Efficient output coupling of intracavity high harmonic generation," Opt. Lett. 33, 1099-1101 (2008).

6. I. Hartl, T. R. Schibli, A. Marcinkevicius, D. C. Yost, D. D. Hudson, M. E. Fermann, and J. Ye, "Cavity-enhanced similariton Yb-fiber laser frequency comb: $3 \times 10^{14} \mathrm{~W} / \mathrm{cm}^{2}$ peak intensity at $136 \mathrm{MHz}$," Opt. Lett. 32, 2870-2872 (2007).

7. M. Hentschel, Z. Cheng, F. Krausz, and Ch. Spielmann, "Generation of 0.1-TW optical pulses with a single-stage Ti:sapphire amplifier at a 1-kHz repetition rate," Appl. Phys. B 70, 161-164 (2000).

8. F. X. Kaertner, W. S. Graves, D. E. Moncton, and F. O. Ilday, US Patent Application Publication US2006/0251217 A1 (2006).

9. F. V. Hartemann, W. J. Brown, D. J. Gibson, S. G. Anderson, A. M. Tremaine, P. T. Springer, A. J. Wootton, E. P. Hartouni, and C. P. J. Barty, "High-energy scaling of Compton scattering light sources," Phys. Rev. Spec. Top. Acc. Beams 8, 100702 (2005).

10. M. Theuer, D. Molter, K. Maki, C. Otani, J. A. Lhuillier, and R. Beigang, "Terahertz generation in an actively controlled femtosecond enhancement cavity," Appl. Phys. Lett. 93, 041119 (2008).

11. A. N. Luiten and J. C. Petersen, "Ultrafast resonant polarization interferometry: Towards the first direct detection of vacuum polarization,” Phys. Rev. A 70, 033801 (2004). 
12. R. G. DeVoe, C. Fabre, K. Jungmann, J. Hoffnagle, and R. G. Brewer, "Precision Optical-Frequency- Difference Measurements," Phys. Rev. A 37, 1802-1805 (1988).

13. C. J. Hood, H. J. Kimble, and J. Ye, "Characterization of high-finesse mirrors: Loss, phase shifts, and mode structure in an optical cavity," Phys. Rev. A 64, 033804 (2001).

14. B. Bernhardt, A. Ozawa, P. Jacquet, M. Jacquey, Y. Kobayashi, T. Udem, R. Holzwarth, G. Guelachvili, T. W. Hänsch, and N. Picqué, "Cavity-enhanced dual-comb spectroscopy," Nat. Photonics 4, 55-57 (2010).

15. M. Thorpe, R. Jones, K. Moll, J. Ye, and R. Lalezari, "Precise measurements of optical cavity dispersion and mirror coating properties via femtosecond combs," Opt. Express 13, 882-888 (2005).

16. A. Schliesser, C. Gohle, T. Udem, and T. W. Hänsch, "Complete characterization of a broadband high-finesse cavity using an optical frequency comb," Opt. Express 14, 5975-5983 (2006).

17. C. Gohle, B. Stein, A. Schliesser, T. Udem, and T. W. Hänsch, "Frequency Comb Vernier Spectroscopy for Broadband, High-Resolution, High-Sensitivity Absorption and Dispersion Spectra," Phys. Rev. Lett. 99, 263902 (2007).

18. T. J. Hammond, A. K. Mills, and D. J. Jones, "Simple method to determine dispersion of high-finesse optical cavities," Opt. Express 17, 8998-9005 (2009).

19. I. Pupeza, T. Eidam, J. Rauschenberger, B. Bernhardt, A. Ozawa, E. Fill, A. Apolonski, Th. Udem, J. Limpert, Z. A. Alahmed, A. M. Azzeer, A. Tünnermann, T. W. Hänsch, and F. Krausz, "Power scaling of a high repetition rate enhancement cavity," Opt. Lett. 12, 2052-2054 (2010).

20. A. P. Kovács, K. Osvay, Zs. Bor, and R. Szipöcs, "Group-delay measurement on laser mirrors by spectrally resolved white-light interferometry," Opt. Lett. 20, 788-790 (1995).

21. D. Meshulach, D. Yelin, and Y. Silberberg, "Real-time spatialspectral interference measurements of ultrashort optical pulses," J. Opt. Soc. Am. B, 14, 2095-2098 (1997).

22. P. Bowlan, P. Gabolde, M. A. Coughlan, R. Trebino, and R. J. Levis, "Measuring the spatiotemporal electric field of ultrashort pulses with high spatial and spectral resolution,” J. Opt. Soc. Am. B 25, A81-A92 (2008).

23. A. Börzsönyi, A. P. Kovács, M. Görbe, and K. Osvay, "Advances and limitations of phase dispersion measurement by spectrally and spatially resolved interferometry," Opt. Commun. 281, 3051-3061 (2008).

24. D. E. Adams, T. A. Planchon, A. Hrin, J. A. Squier, and C. G. Durfee, "Characterization of coupled nonlinear spatiospectral phase following an ultrafast self-focusing interaction,” Opt. Lett. 34, 1294-1296 (2009).

25. K. Osvay, A. Börzsönyi, A. P. Kovács, M. Görbe, G. Kurdi, and M. P. Lalashnikov, "Dispersion of femtosecond laser pulses in beam pipelines from ambient pressure to 0.1 mbar," Appl. Phys. B 87, 457-461 (2007).

26. A. Börzsönyi, Z. Heiner, M. P. Kalashnikov, A. P. Kovács, and K. Osvay, "Dispersion measurement of inert gases and gas mixtures at 800 nm," Appl. Opt. 47, 4856-4863 (2008).

27. K. Osvay, L. Canova, C. Durfee, A. P. Kovács, Á. Börzsönyi, O. Albert, and R. Lopez Martens, "Preservation of the carrier envelope phase during cross-polarized wave generation," Opt. Express 25, 22358-22365 (2009).

28. D. E. Adams, T. A. Planchon, J. A. Squier, and C. G. Durfee, "Spatiotemporal dynamics of cross-polarized wave generation," Opt. Lett. 35, 1115-1117 (2010).

29. K. Osvay, Á. Börzsönyi, Z. Heiner, A. P. Kovács, and M. P. Kalashnikov, "Measurement of Pressure Dependent Nonlinear Refractive Index of Inert Gases,” CLEO 2009, paper CMU7.

30. T. Eidam, F. Röser, O. Schmidt, J. Limpert and A. Tünnermann, " 57 W, 27 fs pulses from a fiber laser system using nonlinear compression," Appl. Phys. B 92, 9-12 (2008).

31. T. W. Hänsch and B. Couillaud, "Laser Frequency Stabilization by Polarization Spectroscopy of a Reflecting Reference Cavity,” Opt. Commun. 35, 441 (1980).

32. C. Froehly, A. Lacourt, and J. C. Vienot, "Time impulse response and time frequency response of optical pupils," Nouv. Rev. Opt. 4, 183-196 (1973).

33. L. Lepetit, G. Cheriaux, and M. Joffre, "Linear techniques of phase measurement by femtosecond spectral interferometry for applications in spectroscopy," J. Opt. Soc. Am. B 12, 24672474 (1995).

34. Th. Udem, R. Holzwarth, and T. W. Hänsch, “Optical frequency metrology,” Nature 16, $233-237$ (2002).

35. E. D. Peck and K. Reeder, “Dispersion of Air,” J. Opt. Soc. Am. 62, 958-962 (1972).

\section{Introduction}

Enhancement cavities are frequently used to increase the efficiency of nonlinear interactions such as second-harmonic generation (SHG). For near-infrared continuous wave lasers, cavities with a finesse approaching $10^{6}$ have been demonstrated [1], albeit with a rather low input power. Recently, efforts to resonantly enhance pulsed lasers have been boosted by the prospect of intracavity high-harmonic generation (HHG) with multi-MHz repetition rates [2-6]. While traditional methods of generating peak intensities exceeding $10^{13} \mathrm{~W} / \mathrm{cm}^{2}$ required for HHG rely on a largely reduced pulse repetition rate (see, for example, [7] and references therein), the intracavity HHG approach can preserve the original repetition rate of the pulsed laser source.

\#132118 - \$15.00 USD Received 22 Jul 2010; revised 30 Sep 2010; accepted 16 Oct 2010; published 1 Dec 2010

(C) 2010 OSA

6 December 2010 / Vol. 18, No. 25 / OPTICS EXPRESS 26185 
Other proposed applications, for which enhancement cavities constitute a very promising approach, are the generation of high-brilliance hard X-rays via inverse Compton scattering [8,9], $\mathrm{THz}$ generation [10] and the detection of the birefringence of vacuum [11].

Enhancing the power of a train of pulses in an optical resonator requires the constructive interference of the intracavity field with the seeding laser field inside the cavity. In the frequency domain this means that the seeding frequency comb and the cavity resonances have to be matched over the optical bandwidth of interest. While the mode spacing of the seeding mode-locked laser can be assumed to be perfectly regular, the cavity free spectral range may be irregular due to dispersion. In a high-finesse cavity, the effect of single-round-trip dispersion is accumulated over many round trips in the resonant state. This typically restricts the power enhancement to a limited spectral range and therefore needs to be precisely measured and compensated for optimum enhancement. Conversely, the same effect enables highly accurate dispersion [12,13] and absorption measurements [14].

In the past few years, several approaches to measuring the intracavity dispersion of femtosecond enhancement cavities have been presented [15-18]. All these techniques rely on a controlled variation of the seeding frequency comb and the subsequent interpretation of the cavity response. In [15] and [16], a limited number of modes of the seeding comb is locked to the cavity. In the locked state, either the repetition frequency [16] or the carrier-envelope-offset frequency [15] of the seeding comb is varied and the intracavity spectrum is recorded. Suitable linear cavity models are fitted to the acquired 2-dimensional data sets. Due to the accumulation of dispersion over many cavity round trips, the group delay dispersion (GDD) can be detected with very good sensitivity and a reproducibility on the order of $1 \mathrm{fs}^{2}$ is achieved. However, the necessary variation of the seeding comb allows only a few modes to resonate at once, such that these methods are limited to the measurement of the linear cavity response. Thus, they are not suitable for the investigation of average- and peak power-related intracavity effects. In [17] and [18] the authors present techniques in which the frequency comb is scanned through the cavity resonances without locking the laser and the cavity. Even if a steady state is not necessarily reached in the cavity, comparable GDD measurement accuracies are reported. However, these methods are also limited to the linear case. For applications of enhancement cavities involving high peak powers, the restriction of dispersion measurement methods to low power levels is rather severe. Peak-power-dependent enhancement behavior has been reported in HHG cavities [4] including a gas target and an XUV output coupler and even in empty cavities [19]. A phase-sensitive measurement method suitable also for regimes beyond linear enhancement is highly desirable and described in this work.

We employ spatially and spectrally resolved interferometry (SSI) [20-29] to measure the phase difference between the intracavity circulating field and the seeding field. Together with the spectrally resolved power enhancement, this measurement provides full information on the cavity response. This method has several major advantages: for phase retrieval over the entire enhanced bandwidth the seeding comb need not be varied. In particular, this enables the investigation of effects related to high intracavity peak power. The experimental setup is simple and the total measurement time is only limited by the cavity build-up time. Once the cavity reaches the steady state, a single-pulse acquisition is sufficient for an accurate cavity response determination. This provides an increased robustness against jitter effects. We present experimental results for both the linear and nonlinear enhancement regimes, demonstrating sensitivities and reproducibilities comparable to those reported in previous works for linear enhancement.

\#132118 - \$15.00 USD Received 22 Jul 2010; revised 30 Sep 2010; accepted 16 Oct 2010; published 1 Dec 2010

(C) 2010 OSA

6 December 2010 / Vol. 18, No. 25 / OPTICS EXPRESS 26186 


\section{Experimental Setup and Phase Measurement Method}

\subsection{The Steady State and the Desired Information}

Consider an enhancement cavity in the steady state. Let $\omega$ denote the angular optical frequency. Let $\widetilde{E}_{i}(\omega)$ and $\widetilde{E}_{c}(\omega)$ denote the complex frequency-domain input electric field from the seeding laser and the intracavity circulating field, respectively, within the enhanced spectral region. The behavior of the cavity is described by the ratio $\widetilde{H}(\omega)$ of the circulating to the incoming field:

$$
\widetilde{H}(\omega)=|\widetilde{H}(\omega)| \exp [j \phi(\omega)]=\frac{\widetilde{E}_{c}(\omega)}{\widetilde{E}_{i}(\omega)},
$$

where $\phi(\omega)$ is the phase of $\widetilde{H}(\omega)$.

There are two qualitatively different regimes of enhancement. In the linear case, $\widetilde{H}(\omega)$ is independent of the circulating field $\widetilde{E}_{c}(\omega)$ and merely a property of the cavity. The second regime of enhancement involves intracavity nonlinear processes. Here, the enhancement behavior and, thus, $\widetilde{H}(\omega)$, depends on the intracavity electric field. Nonlinearities may emerge through the interaction of high peak powers with the cavity optics or some intracavity medium, see e.g. $[4,19]$. In both cases, the complex function $\widetilde{H}(\omega)$ fully describes the response of the cavity to the input electric field $\widetilde{E}_{i}(\omega)$. Therefore, the knowledge of $\widetilde{H}(\omega)$ is highly desirable. However, the single-round-trip propagation properties inside the cavity are often of interest.

The magnitude $|\widetilde{H}(\omega)|$ can be derived from the incident and intracavity power spectra. The latter is accessible through the leakage of one of the highly reflective cavity mirrors (e.g. M3 or M8) knowing its spectral transmission function. To quickly and accurately measure the phase $\phi(\omega)$ of $\widetilde{H}(\omega)$ we apply SSI. The experimental setup is presented in Section 2.2. In Section 2.3 we discuss the derivation of the intracavity single-round-trip propagation properties in the steady state from the measured complex function $\widetilde{H}(\omega)$. Section 2.4 discusses the effect of the correspondence between the single-round-trip intracavity phase and $\phi(\omega)$ on the sensitivity of the interferometric measurement. Section 2.5 addresses the interferometer data processing.

\subsection{The Experimental Setup}

Figure 1 depicts the experimental setup. With the exception of the beamsplitter, the delay line and the imaging spectrometer, the setup is identical to the one described in [19]. The pulses are generated by a passively mode-locked, diode-pumped $\mathrm{Yb}: \mathrm{KYW}$ oscillator and are amplified in a two-stage fiber-based chirped pulse amplifier system. The stretcher and compressor are implemented with transmission gratings. At the output, the $78 \mathrm{MHz}$ repetition rate pulse train reaches up to $50 \mathrm{~W}$ of average power. The central wavelength is $1042 \mathrm{~nm}$. By varying the distance between the two compressor gratings, the pulse duration can be adjusted between $200 \mathrm{fs}$, corresponding to almost bandwidth-limited pulses, and more than $10 \mathrm{ps}$ with a negligible effect on other beam parameters. The laser system is described in detail in [30]. Similar to the systems presented in [2-6], our enhancement cavity is a ring resonator whose round-trip time is adjusted to the inverse of the seeding laser repetition rate. The beam is folded several times for compactness and convenient handling. The cavity is placed inside a vacuum chamber to minimize GDD and losses. All eight dielectric cavity mirrors have low dispersion. The reflectivity of mirrors M2-M8 was determined with a ring-down measurement to be $99.995 \% \pm 20 \mathrm{ppm}$, per mirror. Mirror M1 is the input coupler (IC) of the cavity and has a measured reflectivity of $99.887 \%$, M5 and M6 have a radius of curvature of $150 \mathrm{~mm}$ and enclose a $w_{0}=22 \mu \mathrm{m}$ cavity focus $\left(1 / \mathrm{e}^{2}\right.$-intensity radius calculated for the center of the stability range). The laser transverse mode is matched to the cavity mode with a telescope. As described in [19], a stable lock of the seeding

\#132118 - \$15.00 USD Received 22 Jul 2010; revised 30 Sep 2010; accepted 16 Oct 2010; published 1 Dec 2010 (C) 2010 OSA 6 December 2010 / Vol. 18, No. 25 / OPTICS EXPRESS 26187 


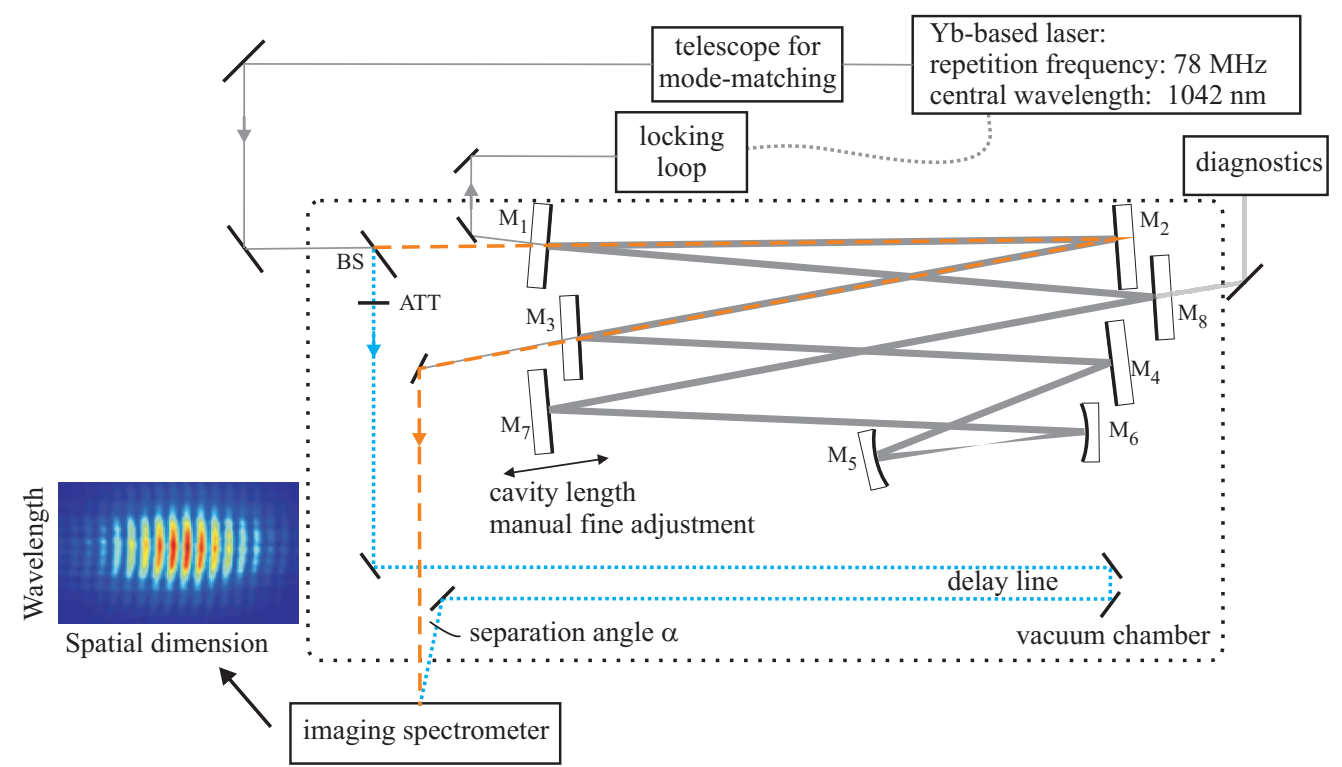

Fig. 1. Experimental setup. BS: beam splitter, blue dotted line: reference beam, orange dashed line: intracavity beam, leaking through the highly reflective mirror M3, ATT: thin neutral density filter attenuation. Diagnostics: photodiode / power meter / spectrometer / autocorrelator.

laser to the enhancement cavity is achieved with the Hänsch-Couillaud scheme [31] controlling the position of an oscillator cavity end mirror mounted on a piezoelectric transducer. For optimum enhancement, an additional coarse adjustment is achieved by manually varying the seed oscillator optical pump power. Typical power enhancement factors range between 1400 and 1800 (see [19]).

To obtain the phase $\phi(\omega)$ of the cavity response function, we use spatially and spectrally resolved interferometry [21-23]. A beamsplitter placed in front of the cavity IC splits off approximately $4 \%$ of the input beam (see the blue dotted line in Fig. 1). This beam constitutes the reference arm of the interferometer. The rest of the input beam is sent to and enhanced in the cavity. A copy of the intracavity circulating pulse leaks through the highly reflective mirror M3 (orange dashed line). This beam constitutes the sample arm of the interferometer. Note that throughout the experiments presented in this paper, we assume the constancy of the transmission of mirror M3 with respect to power. The validity of this assumption is confirmed by the observations in [19]. The transmission of M3 amounts to $1.65 \mathrm{ppm}$. The two arms cross with an angle $\alpha$ at the slit of an imaging spectrometer (model Andor Shamrock 303i using a CCD camera model Andor DV420A-OE). An alternative method for phase retrieval consists in onedimensional spectral interferometry (SI), see e.g. [32,33]. For SI, a second beamsplitter could be used to recombine the two interferometer arms collinearly into one beam impinging on a spectrometer, where a 1-dimensional spectral interference fringe pattern is measured. However, SSI offers a better spectral resolution since it avoids the spectral filtering necessary in SI (see e.g. [22]). The time delay $\Delta \tau$ between the two arms can be adjusted with a delay stage implemented in the reference arm. The spatial-spectral interference of the two arms results in a 2-dimensional fringe pattern, which, together with the spectra from the individual interferometer arms, leads to the complete determination of the complex function $\widetilde{H}(\omega)$. The interferometer setup is very flexible in the sense that it allows for arbitrary modifications of the cavity, under 


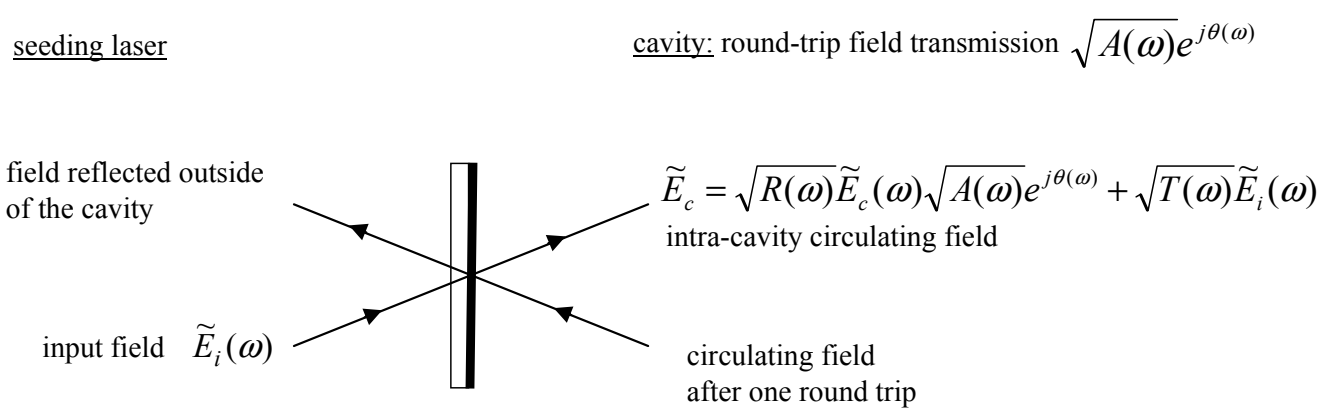

Fig. 2. Electric fields at the input coupler of an enhancement cavity in the steady state.

the constraint that the mirrors M1, M2 and M3 not be displaced. Such modifications may include length and incidence angle variations as well as the exchange of individual mirrors. In particular, the intracavity group delay can be varied by manually displacing mirror M7, i.e. by slightly varying the geometrical cavity length.

To determine $\Delta \tau$, a calibration can be performed while the cavity is off-resonance. For this measurement, we block the cavity beam path without disturbing the propagation in the interferometer sample arm, for instance between mirrors M4 and M5. The delay $\Delta \tau$ is adjusted to 0 by observing the interference fringe spacing. During the measurement, in order to optimize the fringe contrast, the powers in the two interferometer arms are balanced using thin neutral density filters. The GDD imbalance between the two arms can in principle be either corrected using appropriate compensator substrates or numerically removed from the measured phase. In our case, the GDD imbalance owed to a single pass through the substrate of mirror M3 and the neutral density filters amounts to roughly $100 \mathrm{fs}^{2}$. However, as will be discussed in Section 2.4, the effect of the cavity round-trip phase on the phase measured in the sample arm of the interferometer is magnified by more than three orders of magnitude due to the resonant enhancement. Therefore, this imbalance introduces a bias of less than $0.1 \mathrm{fs}^{2}$ for the determined cavity round-trip GDD and is neglected in the measurements presented here.

A mechanical shutter placed in front of the imaging spectrometer controls the integration time of the measurements. Short integration times are favorable to minimize the effects of amplitude and phase jitter on the recorded data. In the measurements presented here, we used an integration time of approximately $0.5 \mathrm{~s}$, which can be further reduced if needed.

A potential source of systematic uncertainty can be the spatial mode mismatch between the cavity and the reference beams, if the input beam phase front is not perfectly matched to that of the excited transverse cavity mode. In the proof-of-principle experiments presented in this paper, we found it quite reasonable to accept some minor ripples in the retrieved phase, caused by this effect (see Section 3.1). To alleviate this issue and to further increase the accuracy of the measurement, a single-mode input beam is preferred, and additional spatial filtering could also be helpful.

\subsection{Intracavity Round-Trip Loss and Accumulated Phase Determination}

Figure 2 shows the complex electric fields at the input coupler (IC) of the enhancement cavity in the steady state. For each enhanced frequency, the single-round-trip power attenuation 
and accumulated phase are denoted by $A(\omega)$ and $\theta(\omega)$, respectively. One cavity round trip of the circulating electric component $\widetilde{E}_{c}(\omega)$ can be completely described by its multiplication by $\sqrt{A(\omega)} \exp [j \theta(\omega)]$. Thus, the desired quantities, fully describing the single-round-trip propagation, are the frequency-resolved quantities $A(\omega)$ and $\theta(\omega)$.

Let $R(\omega)$ denote the IC power reflectivity, such that a reflection on the cavity side at the IC implies the multiplication of the impinging electric field by $\sqrt{R(\omega)}$. Let $T(\omega)$ denote the IC power transmission. Note that we treat $R(\omega)$ and $T(\omega)$ as real functions by including the spectral phase affects at the IC into $\theta(\omega)$. Resonant enhancement requires the constructive interference of the intracavity field reflected by the IC, i.e., $\sqrt{R(\omega)} \widetilde{E}_{c}(\omega) \sqrt{A(\omega)} \exp [j \theta(\omega)]$ with the portion of the input field transmitted through the IC, i.e., $\sqrt{T(\omega)} \widetilde{E}_{i}(\omega)$. In the steady state, the sum of these two interfering fields equals $\widetilde{E}_{c}(\omega)$ :

$$
\begin{aligned}
\widetilde{E}_{c}(\omega) & =\sqrt{R(\omega)} \widetilde{E}_{c}(\omega) \sqrt{A(\omega)} e^{j \theta(\omega)}+\sqrt{T(\omega)} \widetilde{E}_{i}(\omega) \\
\Leftrightarrow \widetilde{H}(\omega)=\frac{\sqrt{T(\omega)}}{\widetilde{E}_{c}(\omega)} & =\frac{\sqrt{E_{i}(\omega)}}{1-\sqrt{R(\omega) A(\omega)} \exp [j \theta(\omega)]} .
\end{aligned}
$$

Equation (3) can be rewritten as:

$$
\sqrt{A(\omega)} e^{j \theta(\omega)}=\frac{1}{\sqrt{R(\omega)}}\left(1-\frac{\sqrt{T(\omega)}}{|\widetilde{H}(\omega)|} e^{-j \phi(\omega)}\right) .
$$

The right-hand side of Eq. (4) contains quantities, which can be independently measured, while the left-hand side contains the desired quantities. Note that Eqs. (3) and (4), evaluated at the frequencies of the seeding comb, hold for both linear and nonlinear enhancement. However, in the nonlinear case the model given by Eq. (3) only describes the effect of the intracavity nonlinearity on the enhancement of the seeding field and it does not allow any direct conclusion on the nature of the nonlinear effect in question. Moreover, the dependence of $R(\omega), T(\omega)$, $A(\omega)$ and / or $\theta(\omega)$ on the intracavity electric field may turn Eq. (3) into a transcendental equation. However, in the steady state, the frequency-dependent quantity $\widetilde{H}(\omega)$, interpreted as the ratio of the circulating to the incoming fields rather than a linear transfer function, allows for a full description of the intracavity propagation in terms of an amplitude attenuation, i.e. loss, and accumulated phase per round trip.

In order to complete the determination of $A(\omega)$ and $\theta(\omega)$ using Eq. (4), we need to address the measurement of $R(\omega)$ and $T(\omega)$. Here, we assume a lossless IC, i.e. $T(\omega)=1-R(\omega)$, as well as the absence of nonlinear processes in the IC. In most practical cases, these assumptions describe the IC behavior very precisely. The IC reflectivity $R$ can be accurately determined with a spectrally resolved ring-down measurement. This technique is well-known and does not require a resonant cavity. Therefore, we assume that the values of $R(\omega)$ and $T(\omega)$ are known.

While the method described here would work with a continuous-spectrum light source as well, only a tiny fraction of its power would be coupled to the resonent cavity. This would result in a low signal-to-noise ratio and, in addition, intracavity strong-field effects could not be studied. Therefore, we illuminate the cavity with a frequency comb, whose modes can be assumed to be equidistant:

$$
\omega_{N}=N \omega_{r}+\omega_{C E} .
$$

Here, $N$ is the comb mode number, $\omega_{r}$ the laser repetition frequency and $\omega_{C E}$ is the carrierenvelope offset frequency [34]. In the context of the laser-cavity lock, the following description of $\theta(\omega)$ is convenient:

$$
\theta(\omega)=\tau_{C} \omega+\psi(\omega)=2 \pi \frac{\omega}{\omega_{r}}+\psi(\omega),
$$

\#132118 - \$15.00 USD

(C) 2010 OSA
Received 22 Jul 2010; revised 30 Sep 2010; accepted 16 Oct 2010; published 1 Dec 2010 6 December 2010 / Vol. 18, No. 25 / OPTICS EXPRESS 26190 
where $\tau_{C}=2 \pi / \omega_{r}$ is the round trip group delay, measured at the optical frequency used to lock the laser, while $\psi(\omega)$ is a residual term, describing the intracavity dispersion. Note that a simple cavity length change, e.g. by slightly displacing mirror M7, influences only the groupdelay term in Eq. (6), while $\psi(\omega)$ remains unaffected. At each frequency of the seeding comb, the cavity round trip phase is:

$$
\theta\left(\omega_{N}\right)=2 \pi N+2 \pi \frac{\omega_{C E}}{\omega_{r}}+\psi\left(\omega_{N}\right)
$$

The first term in the above expression is a multiple of $2 \pi$ for all considered frequencies and, therefore, irrelevant for the analysis that follows. The second term is a constant phase offset, which can be manually tuned by slightly varying the seed oscillator optical pump power (which influences $\omega_{C E}$ ) and / or the cavity length (which influences $\omega_{r}$ ). This degree of freedom allows for the selection of the enhanced spectral range. The cavity group delay dispersion is given by

$$
\operatorname{GDD}(\omega)=\frac{\partial^{2} \theta}{\partial \omega^{2}}=\frac{\partial^{2} \psi}{\partial \omega^{2}}
$$

and does not depend on the chosen offset $\omega_{C E} / \omega_{r}$. Therefore, the GDD information can be obtained by differentiating twice the retrieved intracavity phase for arbitrary offsets. In the particular case of large GDD, leading to a truncation of the enhanced spectrum due to the mismatch of the cavity free spectral range and the seeding frequency comb spacing, the dispersion information over the entire seeding spectrum can be obtained by "stitching" together several measurements for different offsets $\omega_{C E} / \omega_{r}$. In doing so, the knowledge of the phase offset is not necessary.

\subsection{Sensitivity Enhancement due to the Resonant Cavity}

SSI has been successfully employed for sensitive measurements of linear [20,25, 26] and nonlinear [24,27-29] phase effects. The main difference between our technique and earlier works is the resonant enhancement cavity implemented in the interferometer sample arm. This significantly increases both the effect on the pulse caused by the investigated light-matter interaction and the peak / average power available for the interaction in the cavity.

Intracavity phase effects are maximally magnified in the spectral range which is also maximally power-enhanced, which we will refer to as the maximally enhanced range. By varying $\omega_{C E}$ and / or $\omega_{r}$, as explained in the previous section, the maximally enhanced range can be selected within the seed pulse spectrum, see Eq. (7). In this spectral region, $|\theta(\omega)|$ and $|\phi(\omega)| \ll 1$ holds, and we can Taylor-expand equation (3) up to the linear terms of $\theta$ and $\phi$ :

$$
\begin{aligned}
|\widetilde{H}(\omega)| \exp [j \phi(\omega)] & \approx \frac{\sqrt{T(\omega)}}{1-\sqrt{R(\omega) A(\omega)}[1+j \theta(\omega)]} \\
& \approx \frac{\sqrt{T(\omega)}}{1-\sqrt{R(\omega) A(\omega)}}\left[1+j \theta(\omega) \frac{\sqrt{R(\omega) A(\omega)}}{1-\sqrt{R(\omega) A(\omega)}}\right] \\
& \approx \frac{\sqrt{T(\omega)}}{1-\sqrt{R(\omega) A(\omega)}} \exp \left[j \theta(\omega) \frac{\sqrt{R(\omega) A(\omega)}}{1-\sqrt{R(\omega) A(\omega)}}\right] \\
\Rightarrow \phi(\omega) & \approx \frac{\sqrt{R(\omega) A(\omega)}}{1-\sqrt{R(\omega) A(\omega)}} \theta(\omega) .
\end{aligned}
$$

Equation (12) shows that in the maximally enhanced region, the SSI-measured phase of the circulating pulse $\phi(\omega)$ corresponds in good approximation to the the intracavity single-roundtrip phase $\theta(\omega)$, magnified by a factor $\sqrt{R(\omega) A(\omega)} /[1-\sqrt{R(\omega) A(\omega)}]$. This phase magnification factor can be several orders of magnitude for high-finesse cavities. In our experimental

\#132118 - \$15.00 USD

(C) 2010 OSA 
setup, it exceeds 1000 . The magnification from the intracavity round-trip phase $\theta(\omega)$ to the SSI-measured circulating pulse phase $\phi(\omega)$ leads to a significant sensitivity enhancement of our method compared to the conventional SSI.

\subsection{Interferometry Data Processing}

One axis of the 2-dimensional spectrometer data array represents the wavelength or, equivalently, $\omega$. The second dimension, which we denote by $y$, is the spatial axis, along the direction of the spectrometer slit. To determine the magnitude $|\widetilde{H}(\omega)|$, we integrate the individual arm measurements along the $y$-dimension, and obtain the power spectra of the reference and sample arms. The absolute scale of $|\widetilde{H}(\omega)|$ is determined by normalizing the ratio of the integrated areas under the circulating and input spectra to the ratio $P_{\text {circ }} / P_{i n}$, where $P_{\text {circ }}$ and $P_{i n}$ are the measured intracavity circulating power and the input power to the cavity, respectively.

To determine $\phi(\omega)$, we consider the intensity pattern of the interferogram assuming a separation angle $\alpha$ and a time delay $\Delta \tau$ between the interferometer arms and plane phase fronts [21-23]:

$$
I(y, \omega)=I_{R}(y, \omega)+I_{S}(y, \omega)+2 \sqrt{I_{R}(y, \omega) I_{S}(y, \omega)} \cos \left[\phi(\omega)+\omega \Delta \tau+y \frac{\omega}{c} \sin \alpha\right],
$$

where $I_{R}$ and $I_{S}$, represent the intensity distributions of the reference and the sample arms, respectively. Since $I_{R}(y, \omega)$ and $I_{S}(y, \omega)$ can be measured, the cosine term in Eq. (13) can be isolated and calculated from the interferogram $I(y, \omega)$. This term oscillates with the frequency $\frac{\omega}{c} \sin \alpha$ along the $y$-direction and with the frequency $\Delta \tau+\frac{y}{c} \sin \alpha$ along the $\omega$-direction. Note that for the collinear case $(\alpha=0)$ no oscillation takes place along the $y$-direction. An advantage of SSI with $\Delta \tau=0$ and $\alpha \neq 0$ over SI is that the spectral envelope along the $y$-direction usually displays much less structure and is more stable. Therefore, we set the delay $\Delta \tau$ to zero by calibrating the interferometer arms while the cavity is off-resonance. Thus, the isolated cosine term equals $\cos \left[\phi(\omega)+y \frac{\omega}{c} \sin \alpha\right]$. For each $\omega$, this term represents a cosine oscillation in the $y$-direction with a phase offset $\phi(\omega)$. The latter is obtained by a cosine fit for each wavelength in the interferogram. Now, $\theta(\omega)$ and $A(\omega)$ are retrieved directly from Eq. (4).

\section{Experimental Results and Discussion}

\subsection{Dispersion Measurement of Air}

In a first experiment we measured the dispersion of air inside the cavity for several air pressure values. While the pressure-dependent dispersion of air has been accurately measured with SSI before $[25,26]$, the aim of this experiment is to validate our enhancement-cavity-based phase measurement method by comparing the retrieved GDD with the Sellmeier model for the dispersion of air [26,35]. To measure the air pressure we used a Pirani capacitance gauge (Pfeiffer Vacuum, model PCR 260) with a manufacturer specified accuracy between 5\% and $15 \%$ in the pressure range of our experiment. In order to avoid nonlinear effects in the cavity focus, we seeded the cavity with a low input power of $7 \mathrm{~mW}$ and chirped the input pulses to a duration exceeding 2 ps. Since our optical bandwidth is relatively narrow, we restrict the dispersion comparison to the GDD value at $1042 \mathrm{~nm}$. To obtain this value for different air pressure values, we determine $\theta(\omega)$ as described in the previous section and fit a second-order polynomial in $\omega$ to a region corresponding to a few $\mathrm{nm}$ around $1042 \mathrm{~nm}$, similar to [15].

Figure 3 shows the results. In a), b) and c) interferograms for $200 \mathrm{mbar}, 500 \mathrm{mbar}$ and 950 mbar, respectively, are plotted. An increase of the fringe curvature for increasing air pressure can be observed. Part d) shows the intracavity round-trip phase $\theta(\omega)$ including the dispersion from the cavity mirrors and from the intracavity air. To obtain the contribution of air, we

\#132118 - \$15.00 USD Received 22 Jul 2010; revised 30 Sep 2010; accepted 16 Oct 2010; published 1 Dec 2010 (C) 2010 OSA 6 December 2010 / Vol. 18, No. 25 / OPTICS EXPRESS 26192 


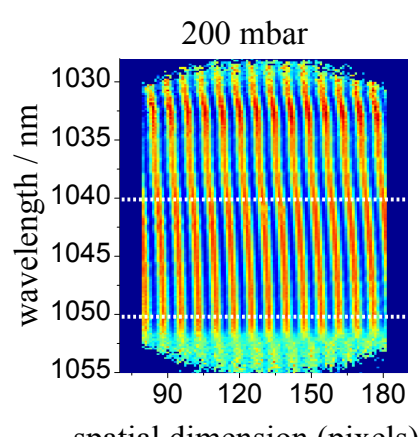

(a)

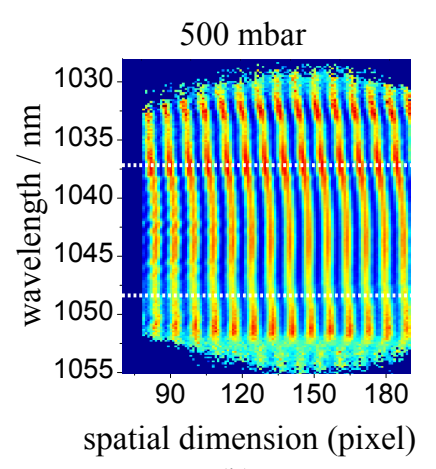

(b)

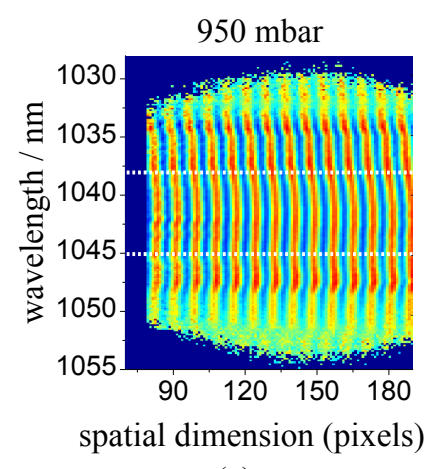

(c)

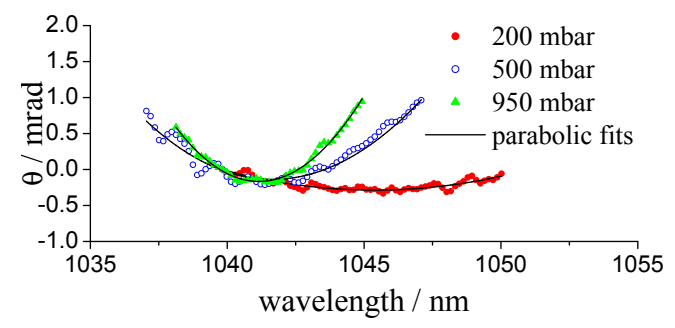

(d)

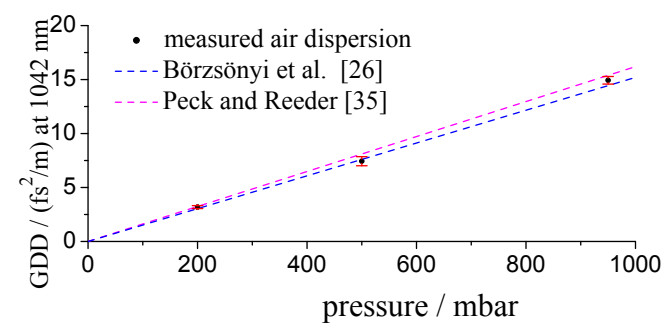

(e)

Fig. 3. Dispersion measurement of the air-filled cavity for different air pressure values. a), b) and c) interferograms for 200 mbar, 500 mbar and 950 mbar, respectively. The white lines indicate the fit range for the second-order polynomial. d) retrieved round-trip phases. e) retrieved air GDD in $\mathrm{fs}^{2} / \mathrm{m}$ after subtracting the empty cavity round trip GDD and comparison with the Sellmeier models $[26,35]$. The error bars show the uncertainty of the quadratic fit, see text for details.

first measure the cavity GDD in vacuum which amounts to $-4.5 \mathrm{fs}^{2}$. The difference between the measured value and the value calculated by summing up the GDD design values specified by the mirror manufacturer for 7 highly reflecting mirrors and the input coupler is less than $1 \mathrm{fs}^{2}$ if we use an optical bandwidth of $\sim 10 \mathrm{~nm}$ to determine the average GDD. The subtraction of the vacuum cavity GDD from the total GDD yields the values plotted in e). The agreement with the Sellmeier model is excellent. The error bars show the $95 \%$ confidence bounds of the quadratic coefficient of the parabolic fit. The calculated relative uncertainties are 4.2\%, 5.7\% and $2.3 \%$ for the 200 mbar, 500 mbar and 950 mbar measurements, respectively. The uncertainty in the fit is mainly given by the oscillating structure on the retrieved phase curves. These oscillations are reproducible for all three measurements, are visible in the interferograms and cause the systematic uncertainty mentioned at the end of Section 2.2.

The parabolic phases in Fig. 3(d) are centered around different wavelength values. This is caused by locking with different constant phase offsets, i.e. different values of the second term in Eq. (7). Nevertheless, the retrieved dispersion values are reproduced, which exemplifies the applicability of our method in the case of relatively large GDD values. 


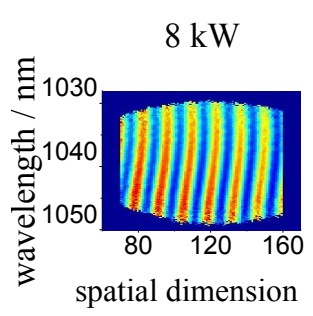

(a)

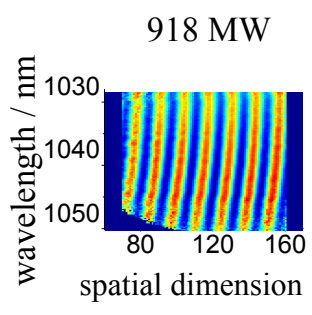

(b)

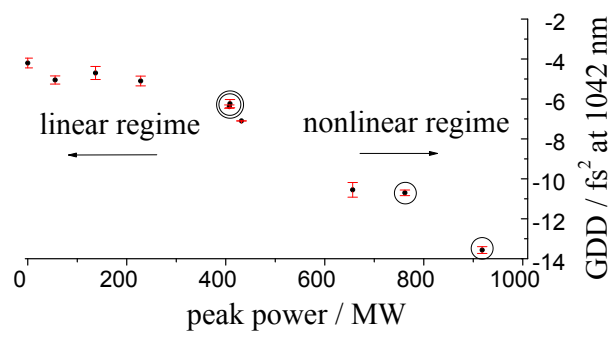

(c)

Fig. 4. Peak-power-dependent round trip GDD measurement of the cavity in vacuum. a) and b) interferograms for peak powers $P_{\text {peak }}=8 \mathrm{~kW}$, and $P_{\text {peak }}=918 \mathrm{MW}$, respectively. c) Retrieved GDD values at $1042 \mathrm{~nm}$ vs. peak power. The three circled measurements are taken at $P_{\text {circ }}=24 \mathrm{~kW}$ and different pulse durations. The two coinciding double-circled measurements are taken at $P_{\text {circ }}=24 \mathrm{~kW}, \tau_{\text {circ }}=650 \mathrm{fs}$ and $P_{\text {circ }}=7 \mathrm{~kW}, \tau_{\text {circ }}=200 \mathrm{fs}$. The error bars (standard deviation of several measurements) are on the order of $0.2 \mathrm{fs}^{2}$.

\subsection{Beyond Linear Enhancement: Peak-Power-Dependent Round Trip GDD Measurement}

The second experiment demonstrates the applicability of our method beyond the linear enhancement regime. In [19] it was reported that the linear enhancement in our cavity is primarily limited by peak-power-dependent, i.e. nonlinear, effects. In order to activate these effects and to measure the resulting round trip GDD, we performed circulating and peak power scaling experiments of the cavity in vacuum in analogy to [19]. By varying the circulating power in the range $0.9 \mathrm{~W} \leq P_{\text {circ }} \leq 23 \mathrm{~kW}$ and the circulating pulse duration in the range $200 \mathrm{fs} \leq \tau_{\text {circ }} \leq 1.5 \mathrm{ps}$, we generated intracavity peak powers between $8 \mathrm{~kW}$ and almost $1 \mathrm{GW}$.

The results are plotted in Fig. 4(a) and 4(b) show interferograms for the cases $P_{\text {peak }}=8 \mathrm{~kW}$, and $P_{\text {peak }}=918 \mathrm{MW}$, respectively. The increased curvature of the interference fringes in part (b) can be recognized. The quantitative results of the GDD determination at $1042 \mathrm{~nm}$ are shown in pannel (c). Up to approximately $300 \mathrm{MW}$, we observe a constant GDD value. For the higher peak powers, a deviation from the linear case is evident. The three circled measurements are taken with a constant circulating power of $P_{\text {circ }}=24 \mathrm{~kW}$ and different pulse durations. They constitute a clear indication that the increased absolute GDD is due to an increase of peak power rather than an increase of the time-averaged circulating power. Another evidence for this is given by the two coinciding double-circled measurements which are taken with $P_{\text {circ }}=24 \mathrm{~kW}$, $\tau_{\text {circ }}=650 \mathrm{fs}$ and $P_{\text {circ }}=7 \mathrm{~kW}, \tau_{\text {circ }}=200 \mathrm{fs}$. The peak power values, at which the determined GDD value starts to deviate from the constant value for the linear regime are in good agreement with the observations in [19]. The exact physical mechanism causing this dispersion requires further study. The error bars indicate a reproducibility of typically $0.2 \mathrm{fs}^{2}$.

\subsection{Phase Distortions Caused by Air Plasma in the Cavity Focus}

Our third experiment addresses qualitatively the highly nonlinear case of a bright air plasma at the intracavity focus. To generate the plasma, we increased the intracavity air pressure up to $8.5 \mathrm{mbar}$ for an input power of $20 \mathrm{~W}$ and an intracavity pulse duration of $650 \mathrm{fs}$. The interferograms for the different pressure values are plotted in Fig. 5. While the autocorrelator measuring the intracavity pulse duration did not indicate any changes over the entire range of pressure values, the interferograms clearly show pressure-dependent phase distortions. A quantitative interpretation of these effects employing an appropriate theoretical model is beyond the scope of this paper. However, this example shows that our method is suited for the sensitive 


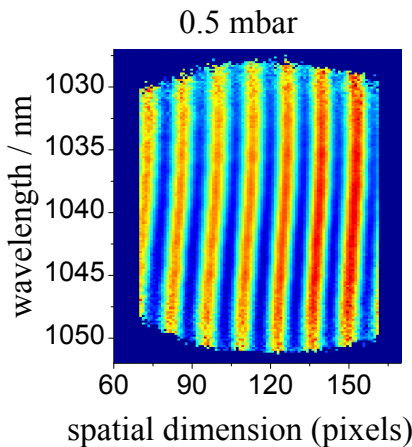

(a)

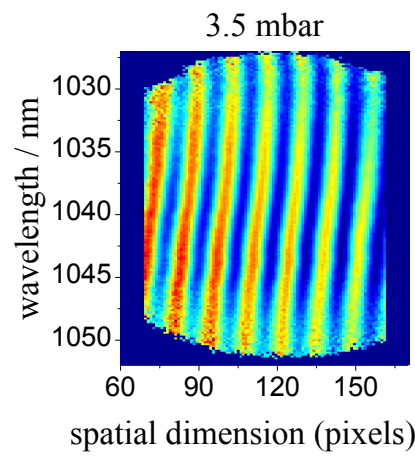

(b)

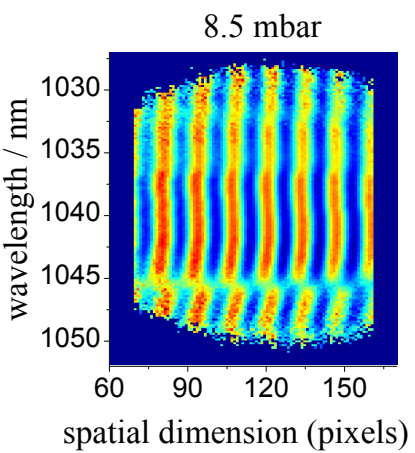

(c)

Fig. 5. Interferograms showing phase distortions caused by an air plasma generated in the cavity focus for an input power of $20 \mathrm{~W}$, a pulse duration of $650 \mathrm{fs}$ and air pressure values of a) 0.5 mbar, b) $3.5 \mathrm{mbar}$ and c) $8.5 \mathrm{mbar}$.

detection and measurement of the influence of highly nonlinear processes on the enhancement behavior of a femtosecond cavity. Conversely, in conjunction with adequate physical models for the nonlinear processes, it offers the prospect of a simple investigation tool with unprecedented precision. With the advent of high-power cavities in the multi-kW regime enhancing sub-picosecond pulses for increasing the net conversion efficiency of nonlinear processes, such as SHG, HHG and THz generation, such studies will become indispensable.

\section{Conclusion}

We have presented a simple and highly sensitive measurement method for the complex ratio of the field circulating in a fully loaded femtosecond enhancement cavity to the incoming field. This method combines the enhancement of power and phase sensitivity provided by a highfinesse cavity in the resonant state with spatial-spectral interferometry, enabling the highly accurate investigation of both linear enhancement regimes and nonlinear light-matter interaction processes at high intensities and repetition rates. In particular, a round trip GDD determination reproducibility better than $1 \mathrm{fs}^{2}$ was demonstrated. The measurement time for the determination of the cavity dispersion over the entire enhanced spectrum amounts to less than 1 second.

The significance of the applicability of this methods beyond the linear enhancement regime is twofold. On the one hand, it allows for the optimization of the cavity for a certain power regime, consisting of e.g. intensity-specific dispersion compensation and / or impedance matching. On the other hand, the measured nonlinear behavior can provide information about the mechanisms causing the nonlinearities, enabling their investigation with a very high accuracy.

\section{Acknowledgements}

This work was supported by the Deutsche Forschungsgemeinschaft (DFG) Cluster of Excellence, Munich Centre for Advanced Photonics (MAP) (www.munichphotonics.de) and by the KORONA Max-Planck-Institut fr Quantenoptik (MPQ)/Fraunhofer Institut fr Lasertechnik (ILT) cooperation. 


\title{
Low-loss VIS/IR-XUV beam splitter for high-power applications
}

\author{
Ioachim Pupeza, ${ }^{*}$ Ernst E. Fill, and Ferenc Krausz \\ Max-Planck-Institut für Quantenoptik, \\ Hans-Kopfermann-Str. 1, 85748 Garching, Germany \\ *ioachim.pupeza@mpq.mpg.de
}

\begin{abstract}
We present a low-loss VIS/IR-XUV beam splitter, suitable for high-power operation. The spatial separation of the VIS/IR and XUV components of a beam is achieved by the wedged top layer of a dielectric multilayer structure, onto which the beam is impinging under Brewster's angle (for VIS/IR). With a fused silica wedge with an angle of $0.5^{\circ}$ we achieve a separation angle of $2.2^{\circ}$ and an IR reflectivity of 0.9995 . Typical XUV reflectivities amount to $0.1-0.2$. The novel element is mechanically robust, exhibiting two major advantages over free-standing Brewster plates: (i) a significant improvement of heat conduction and (ii) easier handling, in particular for high-optical-quality fabrication. The beam splitter could be used as an output coupler for intracavity-generated XUV radiation, promising a boost of the power regime of current MHz-HHG experiments. It is also suited for single-pass experiments and as a beam combiner for pump-probe experiments.
\end{abstract}

(C) 2011 Optical Society of America

OCIS codes: (340.7480) X-rays, soft x-rays, extreme ultraviolet (EUV); (190.2620) Harmonic generation and mixing; (320.7110) Ultrafast nonlinear optics.

\section{References and links}

1. D. Attwood, Soft X-rays and Extreme Ultraviolet Radiation (Cambridge University Press, 1999).

2. P. Jaegle, Coherent Sources of XUV Radiation (Springer, 2006).

3. I. Pupeza, T. Eidam, J. Kaster, B. Bernhardt, J. Rauschenberger, E. E. Fill, Th. Udem, M. F. Kling, J. Limpert, Z. A. Alahmed, A. M. Azzeer, A. Tünnermann, T. W. Hänsch, and F. Krausz, "Power scaling of femtosecond enhancement cavities and high-power applications," Proc. SPIE 7914, 79141I (2011).

4. C. Gohle, Th. Udem, M. Herrmann, J. Rauschenberger, R. Holzwarth, H. A. Schuessler, F. Krausz, and T. W. Hänsch, "A frequency comb in the extreme ultraviolet," Nature 436, 234-237 (2005).

5. R. J. Jones, K. D. Moll, M. J. Thorpe, and J. Ye, "Phase-coherent frequency combs in the vacuum ultraviolet via high-harmonic generation inside a femtosecond enhancement cavity," Phys. Rev. Lett. 94, 193201 (2005).

6. I. Hartl, T. R. Schibli, A. Marcinkevicius, D. C. Yost, D. D. Hudson, M. E. Fermann, and J. Ye, "Cavity-enhanced similariton Yb-fiber laser frequency comb: $3 \times 10^{14} \mathrm{~W} / \mathrm{cm}^{2}$ peak intensity at $136 \mathrm{MHz}$," Opt. Lett. 32, 2870-2872 (2007).

7. A. Ozawa, J. Rauschenberger, C. Gohle, M. Herrmann, D. R. Walker, V. Pervak, A. Fernandez, R. Graf, A. Apolonski, R. Holzwarth, F. Krausz, T. W. Hänsch, and Th. Udem, "High harmonic frequency combs for high resolution spectroscopy," Phys. Rev. Lett. 100, 253901 (2008).

8. D. C. Yost, T. R. Schibli, and J. Ye, "Efficient output coupling of intracavity high harmonic generation," Opt. Lett. 33, 1099-1101 (2008)

9. I. Pupeza, T. Eidam, J. Rauschenberger, B. Bernhardt, A. Ozawa, E. Fill, A. Apolonski, Th. Udem, J. Limpert, Z. A. Alahmed, A. M. Azzeer, A. Tünnermann, T. W. Hänsch, and F. Krausz, "Power scaling of a high repetition rate enhancement cavity," Opt. Lett. 35, 2052-2054 (2010).

10. J. Kaster, I. Pupeza, T. Eidam, C. Jocher, E. Fill, J. Limpert, R. Holzwarth, B. Bernhardt, T. Udem, T. W. Hänsch, A. Tünnermann, and F. Krausz, "Towards MW average powers in ultrafast high-repetition-rate enhancement cavities," High Intensity Lasers and High Field Phenomena (HILAS) Conference, paper HFB4 (2011).

\#146865 - \$15.00 USD Received 29 Apr 2011; revised 30 May 2011; accepted 30 May 2011; published 7 Jun 2011

(C) 2011 OSA

20 June 2011 / Vol. 19, No. 13 / OPTICS EXPRESS 12108 
11. T. Eidam, S. Hanf, E. Seise, T. Andersen, V. T. Gabler, C. Wirth, T. Schreiber, J. Limpert, and A. Tünnermann, "Femtosecond fiber CPA system emitting $830 \mathrm{~W}$ average output power," Opt. Lett. 35, 94-96 (2010).

12. P. Rußbüldt, T. Mans, J. Weitenberg, H. D. Hoffmann, and R. Poprawe, ”Compact diode-pumped $1.1 \mathrm{~kW}$ Yb:YAG Innoslab femtosecond amplifier,' Opt. Lett. 35, 4169-4171 (2010).

13. I. Pupeza, X. Gu, E. Fill, T. Eidam, J. Limpert, A. Tünnermann, F. Krausz, and Th. Udem, "Highly sensitive dispersion measurement of a high-power passive optical resonator using spatial-spectral interferometry," Opt. Express 18, 26784-26195 (2010)

14. K. D. Moll, R. J. Jones, and J. Ye, "Nonlinear dynamics inside femtosecond enhancement cavities," Opt. Express 13, 1672-1678 (2005).

15. R. Paschotta, "Beam quality deterioration of lasers caused by intracavity beam distortions," Opt. Express 14, 6069-6074 (2006).

16. Y.-Y. Yang, F. Sümann, S. Zherebtsov, I. Pupeza, J. Kaster, D. Lehr, H.-J. Fuchs, E.-B. Kley, E. Fill, X.-M. Duan, Z.-S. Zhao, F. Krausz, S. L. Stebbings, and M. F. Kling, "Optimization and characterization of a highly-efficient diffraction nanograting for MHz XUV pulses," Opt. Express 19, 1955-1962 (2011).

17. K. D. Moll, R. J. Jones, and J. Ye, “Output coupling methods for cavity-based high-harmonic generation,” Opt. Express 14, 8189-8197 (2006).

18. A. Ozawa, A. Vernaleken, W. Schneider, I. Gotlibovych, Th. Udem, and T. W. Hänsch, "Non-collinear high harmonic generation: a promising outcoupling method for cavity-assisted XUV generation,” Opt. Express 16, 6233-6239 (2008).

19. J. Weitenberg, P. Rußbüldt, T. Eidam, and I. Pupeza, "Transverse mode tailoring in a high-finesse femtosecond enhancement cavity," Opt. Express 19, 9551-9561 (2011).

20. I. Pupeza, J. Weitenberg, P. Rußbüldt, T. Eidam, J. Limpert, E. Fill, Th. Udem, H.-D. Hoffmann, R. Poprawe, A. Tünnermann, and F. Krausz, "Tailored transverse modes in high-finesse femtosecond enhancement cavities," CLEO 2011, Baltimore, paper QMJ7 (2011).

21. E. D. Palik, Handbook of Optical Constants of Solids (Academic Press, 1991), Vols. 1,2.

22. G. Tempea, V. Yakovlev, B. Bacovic, F. Krausz, and K. Ferencz, “Tilted-front-interface chirped mirrors,” J. Opt. Soc. Am. B 18, 1747-1750 (2001).

23. G. Steinmeyer, "Brewster-angled chirped mirrors for high-fidelity dispersion compensation and bandwidths exceeding one optical octave," Opt. Express 11, 2385-2396 (2003).

24. T. Eidam, F. Röser, O. Schmidt, J. Limpert, and A. Tünnermann, "57 W, 27 fs pulses from a fiber laser system using nonlinear compression,” Appl. Phys. B 92, 9-12 (2008).

\section{Introduction}

Over the past decades, the development of high-power ultrafast laser sources enabled intense research in the field of high-harmonic generation (HHG). Nowadays, for numerous applications in fundamental research, technology and life sciences, involving the generation of broadband extreme ultraviolet (XUV) light [1-3], HHG systems constitute a convenient table-top alternative to synchrotrons. The medium for the highly nonlinear process of HHG is usually a noble gas and intensities on the order of $10^{13} \mathrm{~W} / \mathrm{cm}^{2}$ in the visible and/or infrared range (VIS/IR) are necessary to drive HHG. The conversion efficiency of the fundamental radiation (FR) to the high harmonics $(\mathrm{HH})$ typically lies between $10^{-9}$ and $10^{-6}$, resulting in a significant difference between the power levels of the driving FR and the HH. Moreover, most optical materials exhibit fundamentally different properties in the VIS/IR and XUV ranges. Thus, in the frame of HHG experiments, the design of low-loss optical elements such as beam splitters and combiners is highly challenging.

One of the most prominent state-of-the-art HHG setups, in which this shortcoming plays a major role, relies on the enhancement of high-repetition-rate laser pulses by several orders of magnitude in a high-finesse passive resonator, also called "enhancement cavity" [4-9]. Here, the seeding laser light is interferometrically overlapped with the light circulating inside the cavity. In this manner, energy is continuously coupled to the cavity. In an intracavity focus, the peak intensities for HHG are reached with multi-10-MHz repetition rates. On the one hand, this implies a significant increase of the overall conversion efficiency of the FR to the HH. On the other hand, many applications like frequency comb or coincidence spectroscopy are expected to benefit from the high repetition rates of the intracavity-generated XUV pulses. Recently, the ultrafast MHz-repetition-rate enhancement cavity technology has been scaled up to mJ-

\#146865 - \$15.00 USD Received 29 Apr 2011; revised 30 May 2011; accepted 30 May 2011; published 7 Jun 2011

(C) 2011 OSA

20 June 2011 / Vol. 19, No. 13 / OPTICS EXPRESS 12109 
level circulating pulse energies [9]. New cavity designs [10] seeded by recently developed, powerful laser sources such as reported in $[11,12]$ promise further substantial scaling in the near future. However, to this day, one of the main limitations of the circulating power inside an enhancement cavity for HHG is imposed by the XUV output coupling element, i.e. the optics providing the spatial separation of the FR and the collinearly generated HH. To the best of our knowledge, the circulating power inside femtosecond enhancement cavities including an output coupler $[4,5,7,8]$ is significantly smaller than the power level demonstrated in an empty cavity $[3,9]$.

In this paper we present a low-loss VIS/IR beam splitter suitable for high-power applications. This novel optical element, which we refer to as wedge-on-mirror output coupler (WOMOC), is designed to circumvent limitations of current output couplers for intracavity-generated XUV radiation and to enable a boost of the power regime in intracavity HHG. However, the WOMOC might also be employed to separate the XUV from the FR in single-pass HHG systems or to recombine a VIS/IR and an XUV beam collinearly, e.g. for pump-probe experiments.

The manuscript is structured as follows: in Section 2 the design criteria are discussed and current output coupling methods for intracavity HHG are briefly reviewed, in Section 3 the mode of operation of the WOMOC is described, Section 4 reports on the experimental realization and the characterization of the WOMOC and Section 5 concludes the paper.

\section{Design criteria and existing XUV output couplers for intracavity HHG}

The design criteria discussed in this section are related to the task of coupling out intracavitygenerated XUV radiation. However, they are also desirable in the context of single-pass beam splitting and/or combining.

There are two categories of effects limiting the power enhancement of a frequency comb inside a HHG enhancement cavity. Firstly, intracavity losses attenuate the electric field amplitude upon each round trip. These losses are mainly caused by the cavity optics and the interaction with the nonlinear medium employed for HHG. Typical state-of-the art low-loss, low-dispersion, highly reflecting dielectric mirrors for visible/infrared femtosecond pulses exhibit power loss values of around $50 \mathrm{ppm}$. The interaction with the gas target strongly depends on the parameters of the circulating pulse and of the gas jet. Secondly, in the case of significantly large optical bandwidths, intracavity dispersion affects the circulating electric field and represents an enhancement limitation. On the one hand, the cavity round-trip dispersion leads to a truncation of the optical spectrum coupled to the cavity due to its unevenly spaced resonances in the frequency domain. On the other hand, dispersion also affects the phase of the circulating field. In the high-power case, where nonlinear effects related to the intracavity propagation of the fundamental beam cannot be neglected, both enhancement-limiting mechanisms exhibit intensity-dependent behavior, see e.g. [13, 14]. Consequently, in a high-finesse, highpower enhancement cavity, an output coupling mechanism for intracavity-generated XUV light must satisfy the following conditions:

- C1: The XUV output coupling efficiency should be large.

- C2: The effects of absorption and dispersion of the FR due to the interaction with the output coupler should be small.

- C3: Nonlinear effects in the output coupler caused by the FR should be small.

- C4: The heat caused by the interaction of the FR with the output coupler should be efficiently.

- C5: Other losses, like scattering due to poor optical quality or depolarization of the FR should be small.

\#146865 - \$15.00 USD Received 29 Apr 2011; revised 30 May 2011; accepted 30 May 2011; published 7 Jun 2011

(C) 2011 OSA

20 June 2011 / Vol. 19, No. 13 / OPTICS EXPRESS 12110 
In the first demonstrated HHG enhancement cavity experiments the output coupler was a thin plate, transparent for the FR, placed at Brewster's angle in the cavity beam path as the first optical element after the HHG focus [4,5,7]. The collinear superposition of the FR and the generated XUV hits the surface of the plate. The p-polarized FR is transmitted through the plate while the XUV radiation, for which the refractive index of the plate material is smaller than 1 , experiences total reflection at the surface, is therefore spatially separated from the FR and coupled out of the cavity. Concerning criterion $\mathrm{C} 1$, the XUV reflectivity of materials transparent for the FR under Brewster's angle for the FR typically amounts to 10\%-20\% around $60 \mathrm{~nm}$ and decreases for shorter wavelengths. In order to meet the criteria $\mathrm{C} 2$ and $\mathrm{C} 3$, the thickness of the Brewster plate should be chosen as small as possible. However, decreasing the thickness implies two disadvantages. Firstly, in the vacuum environment necessary for XUV light propagation, the decreased thickness leads to a poor conduction of the heat caused by the absorption in the plate, violating condition $\mathrm{C} 4$. As a consequence, the plate introduces a phase front distortion in the cavity, which is likely to affect the excited mode [15] and the enhancement behavior, and the heating might lead to the damage of the plate. Secondly, mechanical processing and handling becomes more difficult for thin plates, often limiting the feasibility of such plates or leading to poor optical quality, violating criterion C5.

There have been several approaches to circumvent the limitations of the Brewster plate output coupler. In $[8,16]$, a nanostructure is written in the top layer of the cavity mirror following the HHG focus. This optical element acts as a highly reflecting mirror for the FR and as a diffraction grating for the generated XUV, thus providing spatial separation of the $\mathrm{HH}$ from the FR. The output coupling efficiency is comparable to that of a Brewster plate. However, the harmonics coupled out of the cavity are angularly dispersed. For several applications, this is a substantial disadvantage. Moreover, while this technique offers the prospect of scaling to higher circulating powers, the nanostructure might constitute a limiting factor due to undesired effects, e.g. an increased nonlinearity, see [16]. Other output coupling mechanisms, such as non-collinear HHG and output coupling through an aperture on the optical axis of the resonator, optionally using higher-order modes, are proposed in [17-20]. However, these techniques either violate one of the necessary conditions listed above or complicate the cavity setup significantly. To the best of our knowledge, none of these output coupling methods has been successfully experimentally demonstrated to this day.

In conclusion, despite the limitations discussed above, for collinear output coupling of intracavity-generated XUV light from a high-finesse resonator, the free-standing Brewster plate output coupler remains the most convenient method so far. The novel optical element presented in this manuscript combines the low-loss advantages of a thin Brewster plate output coupler with the heat conduction properties, the robustness and the optical quality of a state-of the-art dielectric multilayer mirror.

\section{Description and mode of operation}

\subsection{The concept of the WOMOC}

Figure 1 shows a scheme of the wedge-on-mirror output coupler (WOMOC). It is a dielectric multilayer structure, the top layer of which is wedged at a small angle $\alpha$. The physical principle employed for the spatial separation of the FR and the HH component of the intracavity laser beam is the same as for the Brewster plate discussed in the previous section. The p-polarized FR beam impinges on the wedge surface under the Brewster angle $\phi_{B}$ for the wedge material. The XUV radiation is reflected at the surface of the WOMOC under the angle $\phi_{B}$. The FR penetrates the wedge and propagates towards the next material interface, under an angle of incidence $\phi_{i}$, which is determined by $\phi_{B}$ and $\alpha$. The subsequent dielectric multilayer structure is designed to act as a high reflector for the FR. The reflected portion travels back through the

\#146865 - \$15.00 USD Received 29 Apr 2011; revised 30 May 2011; accepted 30 May 2011; published 7 Jun 2011 (C) 2011 OSA 20 June 2011 / Vol. 19, No. 13 / OPTICS EXPRESS 12111 


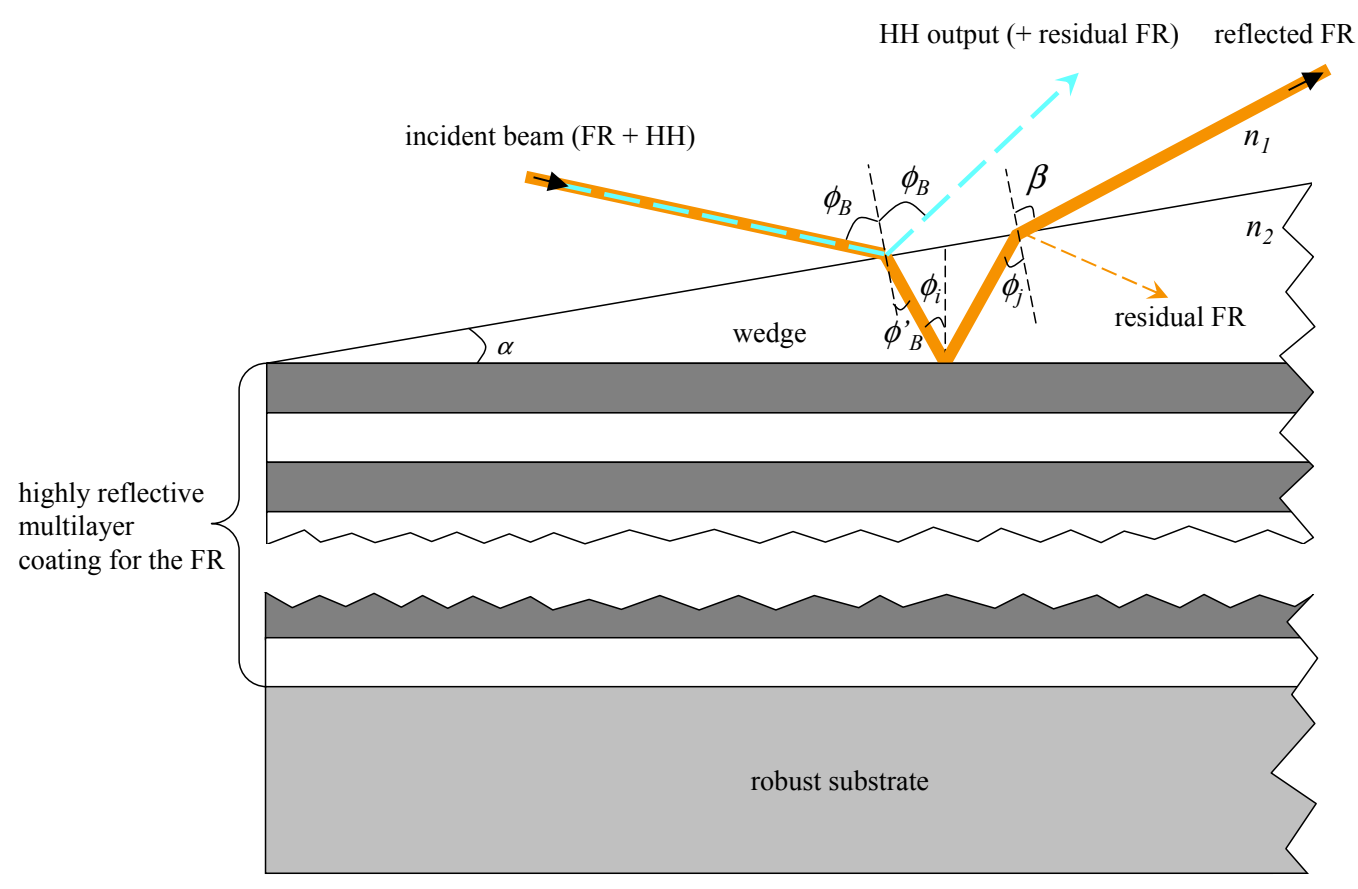

Fig. 1. The WOMOC. FR: fundamental radiation (p-polarized), HH: high harmonics. The multilayer stack underlying the wedge is matched to the adjacent medium $n_{2}$ and for the angle of incidence $\phi_{i}$.

wedged layer and hits its surface under an angle $\phi_{j}$. If $\alpha$ and the fundamental beam divergence are small, the deviation of $\phi_{j}$ from the Brewster angle for this interface is small, such that (i) the overall losses of the fundamental beam upon reflection at the WOMOC are small and (ii) the fundamental is reflected by the WOMOC under a slightly larger angle $\beta>\phi_{B}$ than the $\mathrm{HH}$, providing the spatial separation of the two components. In the following, we derive the separation angle between the FR and the HH beams analytically.

Let the real part of the refractive index of the surrounding medium be $n_{1}$ (typically vacuum) and of the wedge material $n_{2}$. Then, Brewster's angle for the p-polarized fundamental beam is given by:

$$
\phi_{B}=\arctan \frac{n_{2}}{n_{1}} .
$$

The $\mathrm{HH}$ radiation will be reflected by the WOMOC under this angle. By Snell's law, the refraction angle for the beam transmitted through the $n_{1}-n_{2}$-interface equals:

$$
\phi_{B}^{\prime}=\arcsin \left(\frac{n_{1}}{n_{2}} \cdot \sin \phi_{B}\right) .
$$

The incidence angle $\phi_{i}$ of the fundamental beam on the subsequent layer is:

$$
\phi_{i}=\phi_{B}^{\prime}+\alpha .
$$

Figure 1 shows that the outgoing angle $\phi_{j}$ of the reflected fundamental beam is:

$$
\phi_{j}=\phi_{B}^{\prime}+2 \alpha .
$$

\#146865 - \$15.00 USD Received 29 Apr 2011; revised 30 May 2011; accepted 30 May 2011; published 7 Jun 2011 (C) 2011 OSA 20 June 2011 / Vol. 19, No. 13 / OPTICS EXPRESS 12112 
Applying Snell's law again we obtain the following output angle of the fundamental beam with respect to the WOMOC surface normal:

$$
\begin{aligned}
\beta & =\arcsin \left(\frac{n_{2}}{n_{1}} \cdot \sin \phi_{j}\right) \\
& =\arcsin \left\{\frac{n_{2}}{n_{1}} \cdot \sin \left[\arcsin \left(\frac{n_{2}}{n_{1}} \cdot \sin \left(\arctan \frac{n_{2}}{n_{1}}\right)\right)+2 \alpha\right]\right\} .
\end{aligned}
$$

Now we can compute the separation angle between the HH beam and the FR beam upon reflection at the WOMOC:

$$
\delta=\beta-\phi_{B}
$$

Because the wedge layer is very thin, we can assume for simplicity that both the HH and the FR beams leave the WOMOC at the same position, with an angle $\delta$ between them. After a propagation distance $L$ the spatial separation $d$ between the centers of the two beams equals:

$$
d=L \cdot \tan (\delta)
$$

The reflection power losses $R_{\text {loss }}$ of the FR at the WOMOC owed to the deviation from Brewster's angle when the beam exits the wedge, assuming a collimated beam, can be calculated by means of the Fresnel equations and are given by the following expression:

$$
R_{\text {loss }}=\left|\frac{n_{2} \sqrt{1-\left(\frac{n_{2}}{n_{1}} \sin \phi_{j}\right)^{2}}-n_{1} \cos \phi_{j}}{n_{2} \sqrt{1-\left(\frac{n_{2}}{n_{1}} \sin \phi_{j}\right)^{2}}+n_{1} \cos \phi_{j}}\right|^{2} .
$$

Note that here we have chosen Brewster's angle as the angle of incidence of the incoming beam for the simplicity of the derivation. In fact, choosing a slightly different angle of incidence can even decrease the FR reflection losses while the beam separation angle $\delta$ stays nearly constant. The special cases in which (i) the incident beam $(\mathrm{FR}+\mathrm{HH})$ strikes the WOMOC surface under Brewster's angle (i.e. the case discussed here) and (ii) the FR beam leaving the wedge exits under Brewster's angle exhibit two particularities, which might be desirable: in case (i) the residual FR co-propagating with the HH beam upon reflection at the WOMOC is minimized. In case (ii), the residual FR propagating inside the wedge is minimized.

\subsection{A quantitative example}

As a quantitative example, we consider $\mathrm{SiO}_{2}$ (fused silica) as the material of the wedge, with the refractive index $n_{2}=1.47$ for the FR (optical dispersion is neglected in this example). With $n_{1}=1$ we obtain the Brewster angle $\phi_{B}=55.77^{\circ}$. Figure 2 shows the separation angle $\delta$ as well as the losses $R_{\text {loss }}$ as functions of the wedge angle $\alpha$. The wedge-induced loss $R_{\text {loss }}$ limits the theoretically achievable power enhancement in an enhancement cavity containing this element to $1 / R_{\text {loss }}$ (see e.g. [4] for details). The XUV reflectivity at $60 \mathrm{~nm}$ amounts to $\sim 10 \%$ [21].

Figure 3 shows the region around the focus of a standard-design bow-tie enhancement cavity for HHG, with an implemented WOMOC. Note that for visualization purposes, the figure is not to scale. In this example, we consider a $78 \mathrm{MHz}$ repetition rate infrared FR cavity, similar to the one presented in [9]. However, in contrast to [9], asymmetric focusing is used to increase the distance from the cavity focus to the subsequent curved mirror, facilitating the inclusion of the WOMOC. The radii of curvature of the spherical mirrors $C_{1}$ and $C_{2}$ are 100 and $200 \mathrm{~mm}$, respectively. In the cavity stability range center this results in a focus radius of $22 \mu \mathrm{m}$. The WOMOC is placed $50 \mathrm{~mm}$ after the focus. The FR beam radii $\left(1 / e^{2}\right.$ of the intensity) at mirror

\#146865 - \$15.00 USD Received 29 Apr 2011; revised 30 May 2011; accepted 30 May 2011; published 7 Jun 2011 (C) 2011 OSA 20 June 2011 / Vol. 19, No. 13 / OPTICS EXPRESS 12113 


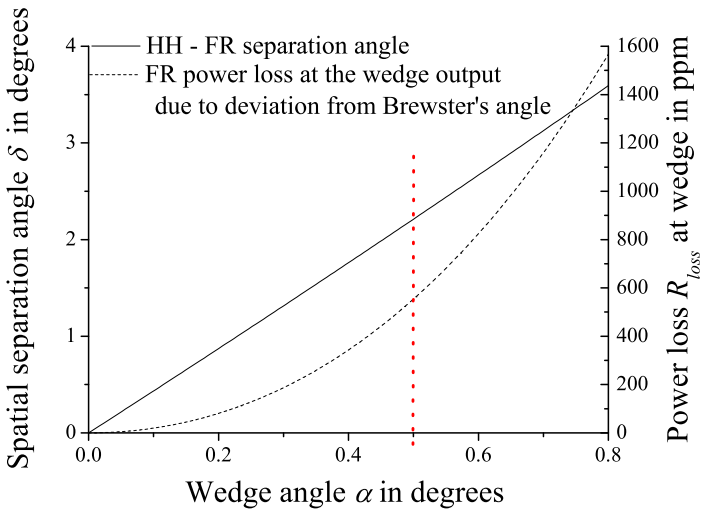

Fig. 2. Spatial separation angle $\delta$ between the FR and the HH upon reflection at the WOMOC and FR losses $R_{\text {loss }}$ at the exit of the wedge due to deviation from Brewster's angle as functions of the wedge angle $\alpha$ for the wedge material $\mathrm{SiO}_{2}$ (refractive index=1.47). The dotted line (red) indicates the values for the prototype presented in Section 4.

$\mathrm{C}_{1}$, at the WOMOC and at mirror $\mathrm{C}_{2}$ are $0.7,0.7$ and $1.6 \mathrm{~mm}$, respectively. The incidence angle on the WOMOC surface corresponds to the Brewster angle $\phi_{B}$. We choose a wedge angle $\alpha=0.5^{\circ}$, leading to a separation angle $\delta=2.2^{\circ}$ between the XUV and the FR. On the one hand, this small wedge angle enables the realization of a thin wedge layer, implying low losses and nonlinearities. On the other hand, after propagating $50 \mathrm{~mm}$ from the WOMOC to mirror $\mathrm{C}_{2}$, the centers of the two beams are $\sim 2 \mathrm{~mm}$ apart, making the XUV output coupling possible. The theoretical losses $R_{\text {loss }}$ amount to $554 \mathrm{ppm}$, allowing for a power enhancement exceeding 1800 .

In order to increase the distance from the WOMOC to the next mirror for a certain wedge angle and, thus, increase the spatial separation of the FR and $\mathrm{HH}$ at the position of this mirror, additional curved mirrors can be implemented in the cavity. Alternatively, the curved mirror after the HHG focus could be placed under oblique incidence, as can be done e.g. with parabolic mirrors. This mirror could on the one hand nearly collimate the FR beam and on the other hand reflect a substantial portion of the generated XUV due to the large angle of incidence. Placing a WOMOC after this mirror could therefore bare several advantages over placing it between the focus and this mirror. Firstly, the FR is (nearly) collimated, implying Brewster angle incidence over the entire beam profile. Secondly, the spatial constraint imposed to the separation angle $\delta$, and, therefore to the wedge angle $\alpha$ by the mirror following the WOMOC can be relaxed if the mirror following the WOMOC is placed at a large distance.

\subsection{Advantages of the WOMOC}

While the spatial separation of the FR and the HH at the surface of the WOMOC relies on the same physical principle as in the case of the free-standing Brewster plate output coupler and the output coupling efficiencies of both methods are identical, the WOMOC exhibits several advantages which are discussed in the following.

The WOMOC is a robust, compact optical element enabling easy handling and processing in contrast to free-standing foils/plates. This allows for the production of a very thin wedged layer, introducing low additional absorption, heat production and other losses to the FR. A particular advantage of the small material thickness is the reduction of dispersion and nonlinear effects. These properties are particularly valuable in the case of resonant cavities. Furthermore, it facilitates good control of the optical quality of the surface, even for a very thin wedged

\#146865 - \$15.00 USD Received 29 Apr 2011; revised 30 May 2011; accepted 30 May 2011; published 7 Jun 2011

(C) 2011 OSA

20 June 2011 / Vol. 19, No. 13 / OPTICS EXPRESS 12114 


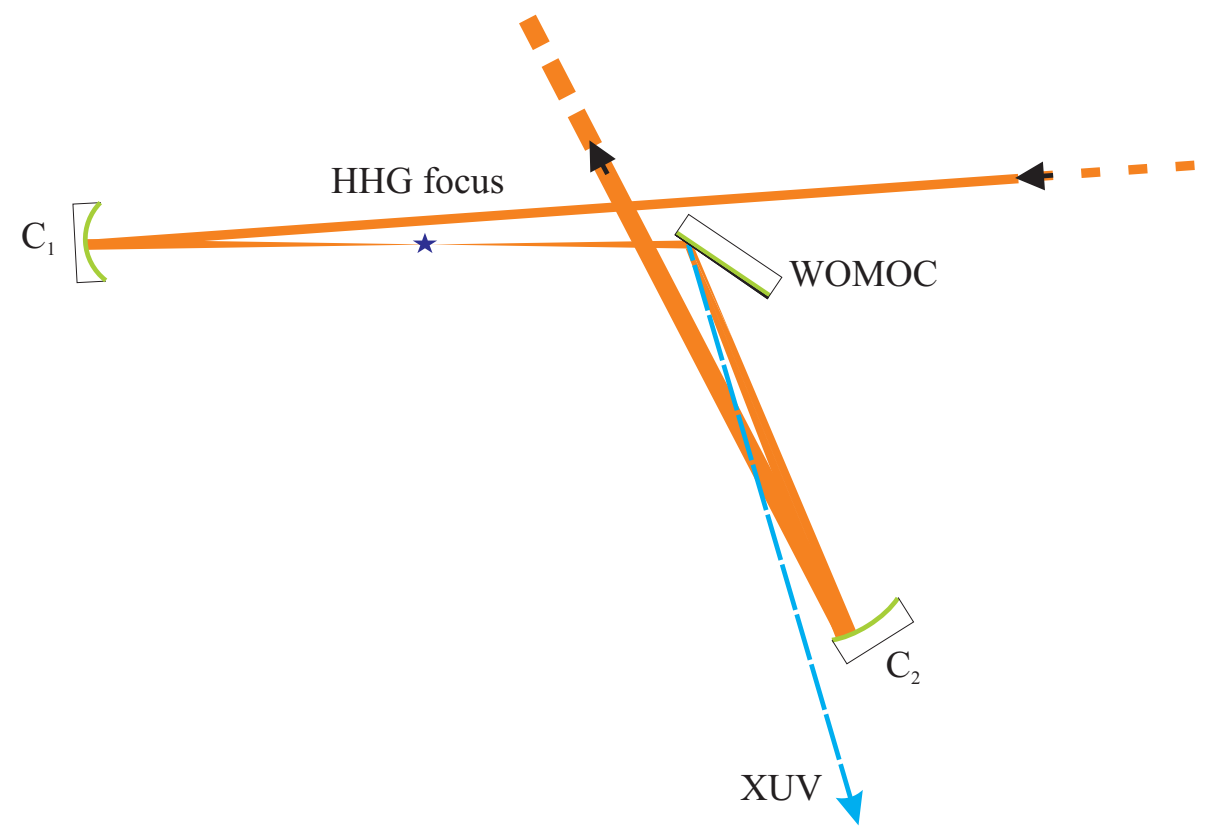

Fig. 3. WOMOC implementation in a standard-design bow-tie ring cavity. Mirrors $\mathrm{C}_{1}$ and $\mathrm{C}_{2}$ are the curved mirrors with radii of curvature equal to 100 and $200 \mathrm{~mm}$, respectively. All other cavity mirrors can be plane mirrors and are left out here for simplicity. High harmonics are generated collinearly with the laser in the focus.

layer. Also, this robust element is easier to align, to handle and to mount and mechanically more stable than a free-standing thin plate.

One surface of the wedged layer, through which the FR passes, is attached to a large optical element, enabling the efficient transport of heat generated by the absorption in the wedge material, away from the illuminated area. A thorough investigation of the heat conduction properties exceeds the scope of this paper. However, the experimental results presented in the subsequent section illustrate the improved heat conduction. Recent experimental results in our group indicate that thermal effects in the free-standing Brewster plate represent the current limitation of power scaling in an enhancement cavity including such an output coupler. Thus, the increased efficiency of the heat transport is expected to boost the achievable intracavity power levels.

While the FR passes through the wedge, it accumulates group delay dispersion. Knowing the material and geometric properties of the wedge, this dispersion can be estimated and compensated by the mirror coating design. By a slight transverse translation of the WOMOC, the length of the FR beam path inside the wedge can be varied, such that the incidence angles are not affected. In this manner, a fine tuning of the FR dispersion upon reflection at the WOMOC can be achieved. The significance of this property increases with shorter pulses. For the determination of the dispersion during this fine tuning process, the method presented in [13] can be employed. Furthermore, both the wedged upper layer and the Brewster angle incidence represent advantages from the point of view of the low-dispersion design criterion, see [22] and [23], respectively. 


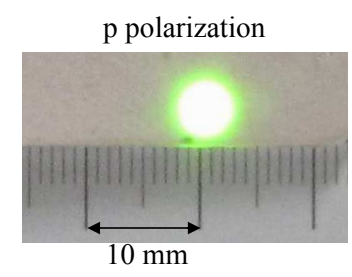

(a)

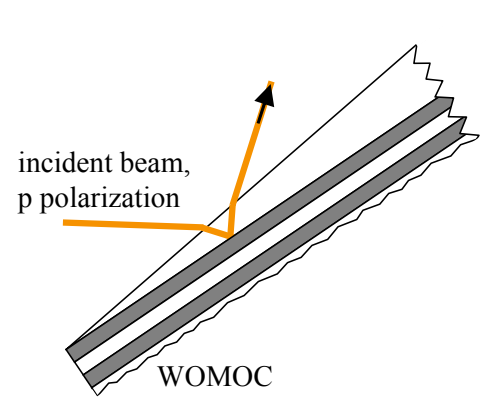

(b)

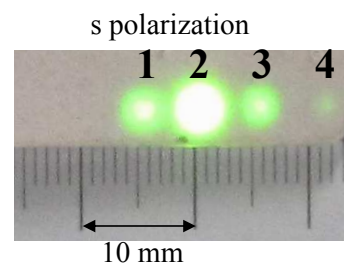

(c)

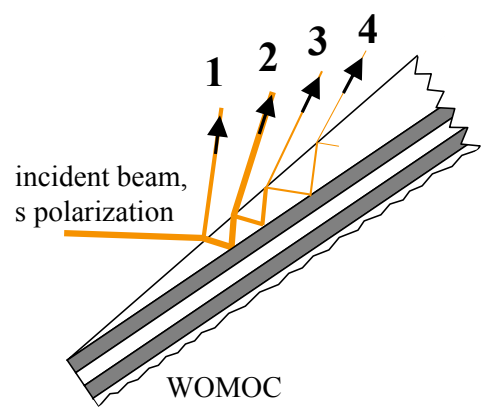

(d)

Fig. 4. Experimental demonstration of the spatial separation property of the WOMOC: reflection of a beam impinging under Brewster's angle on the surface of the WOMOC, after a propagation of $100 \mathrm{~mm}$. (a) and (b): visualization of the beam with an IR-sensitive card and sketch of beam propagation path in the WOMOC, respectively, for p-polarized light. (c) and (d): s-polarized light. Spot 1: direct reflection from the wedge surface, spot 2 beam penetrating the wedge and reflected at the multilayer, spots 3 and 4 are cause by multiple reflections inside the wedge.

\section{Experimental results}

\subsection{Technical realization}

To demonstrate the technical feasibility of the WOMOC we produced a prototype according to the example considered in Section 3.2. Firstly, a standard low-loss dielectric quarter-wave stack coating for the highly reflective mirror underlying the wedged top layer was designed. The reflectivity was maximized for the central wavelength of $1040 \mathrm{~nm}$ and the coating design was matched to the refractive index of the wedge material, i.e. $n_{2}=1.47$ and to the inner angle of incidence of $\phi_{i}=34.2^{\circ}$. This coating was ion-beam sputtered onto a standard 1-inchdiameter fused silica substrate. Secondly, a $0.5 \mathrm{~mm}$ fused silica plate with parallel surfaces was optically contacted to the surface of the coated mirror. Thirdly, to obtain the wedge and optimum optical quality of the surface, the top layer was lapped and polished under an angle $\alpha=0.5^{\circ}$ over the entire surface. The small wedge angle implies a short propagation length through the wedge material. Therefore, no further dispersion compensation was accounted for in the coating design. 


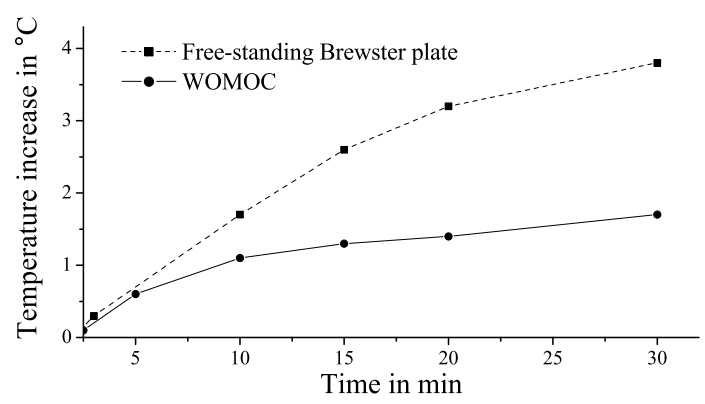

Fig. 5. Comparison of the evolution of the temperature increase from room temperature measured at the surface of a free-standing Brewster plate and the surface of the WOMOC. Both were illuminated with a 40-W beam under Brewster's angle in a vacuum chamber.

\subsection{Characterization}

In a first experiment, we tested the spatial separation property of the prototype. The linearly polarized output beam of our seeding laser [24] with a central wavelength of $1040 \mathrm{~nm}$ and with an average power of $0.5 \mathrm{~W}$ is sent to the surface of the WOMOC under $\phi_{B}=55.8^{\circ}$ angle of incidence. Figure 4 shows the beam path as well as the laser spots detected with an IR-sensitive card after propagating $100 \mathrm{~mm}$ away from the WOMOC surface, for $\mathrm{p}$ and for s polarization. For p polarization we observe a single reflection, see Figs. 4(a) and 4(b). In order to detect the direct reflection from the wedge surface, we rotated the polarization of the incident beam by 90 degrees with a half-wave plate. We observe several spots, see Figs. 4(c) and 4(d). The spot numbered by 1 is caused by direct reflection from the wedge surface. Spot 2 stems from the portion of the beam which penetrates the wedge layer, is reflected by the multilayer coating and exits the wedge. Before exiting the wedge, a portion of this beam is reflected back into the wedge layer at the wedge-air interface. Subsequently, this portion is again reflected by the multilayer and is split into the beam leading to spot 3 and into another back reflection from the wedge-air interface. This reflection pattern is repeated and gives rise to spot 4 . On the righthand side of spot 4, further spots with decreasing intensities can be detected (not shown here). A collinear combination of a p-polarized VIS/IR beam and its HH, incident under $\phi_{B}=55.8^{\circ}$ on the WOMOC surface would be split into a single reflection of the FR, indicated by the single spot in Fig. 4(a) and located at the position of spot 2 in Fig. 4(c) and into the XUV beam, located at the position of spot 1 . The distance between the centers of the two spots is $\sim 4 \mathrm{~mm}$, which is in excellent agreement with the example discussed in Section 3.2.

To determine the reflection losses for the p-polarized FR impinging on the WOMOC surface under $\phi_{B}=55.8^{\circ}$, we performed a standard ring-down measurement. Over the entire bandwidth of our seeding laser, losses in the range 500-600 ppm were measured, which is in excellent agreement with the value calculated in Section 3.2, see Fig. 2.

The last experiment illustrates the heat conduction improvement of the WOMOC compared to a free-standing Brewster plate, with a comparable thickness. The WOMOC and a $100 \mu \mathrm{m}$ thin fused silica plate (same material as the wedge layer of the WOMOC) were illuminated with $40 \mathrm{~W}$ average power. Both elements were mounted at Brewster's angle in a vacuum chamber. The collimated beam was p-polarized and had a radius of $1.8 \mathrm{~mm}$. The light transmitted through and reflected by the Brewster plate and the WOMOC, respectively, was steered out of the chamber to avoid residual heating. The pressure in the vacuum chamber was $<10^{-1}$ mbar. With an IR thermometer (Voltcraft IR-1001A) the temperature of the surface of the optical el- 
ements was measured through a zinc selenide vacuum chamber window, which is transparent for mid-infrared heat radiation. The Brewster plate was mounted by clamping its lower edge mechanically and the WOMOC was mounted in a standard mirror mount. Figure 5 shows the temperature increase from room temperature measured over a time of 30 minutes and clearly indicates that the WOMOC exhibits superior heat conduction. In the case of a high-power application, such as XUV output coupling from an enhancement cavity, with average powers in the multi-kW range, this advantage is expected to play a decisive role. It should also be mentioned that the heat transport away from the illuminated spot can be improved for both optical elements by means of their mounting. A detailed analysis of the heat transport mechanisms and an optimization of the mechanical mounts exceeds the scope of this paper such that the results plotted in Fig. 5 should be interpreted qualitatively rather than quantitatively.

\section{Conclusion}

The concept, realization and experimental characterization of a novel low-loss VIS/IR-XUV beam splitter, suitable for high-power applications, is presented. The spatial separation of the XUV from the VIS/IR spectral components is achieved by the wedged top layer of a dielectric multilayer structure and relies on the same physical mechanism as in the case of a free-standing Brewster plate. However, the robustness of our beam splitter leads to two advantages over a freestanding thin Brewster plate. On the one hand, the heat conduction is significantly improved, allowing for operation at higher powers. On the other hand, mechanical handling becomes easier. In particular, this allows for high-optical-quality fabrication, even for an extremely thin top layer. We demonstrate experimentally the spatial separation, the high reflectivity for the fundamental radiation as well as the improved heat conduction. With a fused silica wedge with an angle of only $0.5^{\circ}$ we achieve a spatial separation angle of $2.2^{\circ}$, which is enough to couple out high harmonics from a standard-design enhancement cavity. The measured reflectivity for the fundamental radiation is 0.9995 . The reflectivity in the XUV spectral region is subject to further investigation. However, since a better optical quality is achievable due to the element's robustness, the XUV reflectivity is expected to surpass the value of a free-standing Brewster plate from the same material as the wedge layer.

The beam splitter was primarily designed as a wedge-on-mirror output coupler (WOMOC) for collinear output coupling of intracavity-generated high-harmonic radiation, circumventing limitations of current methods. It is expected to enable a significant boost of the power regime in intracavity $\mathrm{HHG}$ experiments in the near future. In addition, the WOMOC might also be employed to separate the XUV from the FR in single-pass HHG systems or to combine a VIS/IR and an XUV beam collinearly, e.g. for pump-probe experiments.

\section{Acknowledgments}

The prototype was fabricated by the company Layertec GmbH. The authors gratefully acknowledge the support of Dr. Peter Zimmermann from Layertec and wish to thank their colleagues: Jan Kaster for preparing the vacuum chamber for the heat measurement experiment, Vladimir Pervak, Oleg Pronin and Alexander Apolonski for helpful discussions. This work was supported by the Deutsche Forschungsgemeinschaft (DFG) Cluster of Excellence, Munich Centre for Advanced Photonics (MAP) (www.munichphotonics.de) and by the BMBF via PhoNa (Photonische Nanomaterialien).

\#146865 - \$15.00 USD Received 29 Apr 2011; revised 30 May 2011; accepted 30 May 2011; published 7 Jun 2011

(C) 2011 OSA

20 June 2011 / Vol. 19, No. 13 / OPTICS EXPRESS 12118 


\title{
Optimization and characterization of a highly- efficient diffraction nanograting for $\mathrm{MHz}$ XUV pulses
}

\author{
Ying-Ying Yang, ${ }^{1,2,3}$ Frederik Süßmann, ${ }^{1,4}$ Sergey Zherebtsov, ${ }^{1}$ Ioachim Pupeza, ${ }^{1,4}$ \\ Jan Kaster, ${ }^{1,4}$ Dennis Lehr, ${ }^{5}$ Hans-Jörg Fuchs, ${ }^{5}$ Ernst-Bernhard Kley, ${ }^{5}$ Ernst Fill, ${ }^{1,4}$ \\ Xuan-Ming Duan, ${ }^{2}$ Zhen-Sheng Zhao, ${ }^{2}$ Ferenc Krausz, ${ }^{1,4}$ Sarah L. Stebbings, ${ }^{1}$ \\ and Matthias F. Kling ${ }^{1, *}$ \\ ${ }^{I}$ Max-Planck-Institut für Quantenoptik, Hans-Kopfermann-Straße 1, 85748 Garching, Germany \\ ${ }^{2}$ Technical Institute of Physics and Chemistry, Chinese Academy of Sciences, 100190 Beijing, China \\ ${ }^{3}$ Graduate School of the Chinese Academy of Sciences, 100190 Beijing, China \\ ${ }^{4}$ Ludwig-Maximilians-Universität München, Am Coulombwall 1, 85748 Garching, Germany \\ ${ }^{5}$ Friedrich-Schiller-Universität Jena, 07737 Jena, Germany \\ *matthias.kling@mpq.mpg.de
}

\begin{abstract}
We designed, fabricated and characterized a nano-periodical highly-efficient blazed grating for extreme-ultraviolet (XUV) radiation. The grating was optimized by the rigorous coupled-wave analysis method (RCWA) and milled into the top layer of a highly-reflective mirror for IR light. The XUV diffraction efficiency was determined to be around $20 \%$ in the range from 35.5 to $79.2 \mathrm{~nm}$. The effects of the nanograting on the reflectivity of the IR light and non-linear effects introduced by the nanograting have been measured and are discussed.

C2011 Optical Society of America

OCIS codes: (050.1950) Diffraction gratings; (340.7480) X-rays, soft x-rays, extreme ultraviolet.
\end{abstract}

\section{References and links}

1. P. B. Corkum, "Plasma perspective on strong field multiphoton ionization," Phys. Rev. Lett. 71(13), 1994-1997 (1993).

2. C. Spielmann, N. H. Burnett, S. Sartania, R. Koppitsch, M. Schnürer, C. Kan, M. Lenzner, P. Wobrauschek, and F. Krausz, "Generation of Coherent X-rays in the Water Window Using 5-Femtosecond Laser Pulses," Science 278(5338), 661-664 (1997).

3. M. I. Stockman, M. F. Kling, U. Kleineberg, and F. Krausz, "Attosecond nanoplasmonic-field microscope,” Nat. Photonics 1(9), 539-544 (2007).

4. C. Gohle, T. Udem, M. Herrmann, J. Rauschenberger, R. Holzwarth, H. A. Schuessler, F. Krausz, and T. W. Hänsch, "A frequency comb in the extreme ultraviolet," Nature 436(7048), 234-237 (2005).

5. R. J. Jones, K. D. Moll, M. J. Thorpe, and J. Ye, "Phase-coherent frequency combs in the vacuum ultraviolet via high-harmonic generation inside a femtosecond enhancement cavity," Phys. Rev. Lett. 94(19), 193201 (2005).

6. A. Ozawa, J. Rauschenberger, Ch. Gohle, M. Herrmann, D. R. Walker, V. Pervak, A. Fernandez, R. Graf, A. Apolonski, R. Holzwarth, F. Krausz, T. W. Hänsch, and T. Udem, "High harmonic frequency combs for high resolution spectroscopy," Phys. Rev. Lett. 100(25), 253901 (2008).

7. D. C. Yost, T. R. Schibli, and J. Ye, "Efficient output coupling of intracavity high-harmonic generation," Opt. Lett. 33(10), 1099-1101 (2008).

8. I. Pupeza, T. Eidam, J. Rauschenberger, B. Bernhardt, A. Ozawa, E. Fill, A. Apolonski, T. Udem, J. Limpert, Z. A. Alahmed, A. M. Azzeer, A. Tünnermann, T. W. Hänsch, and F. Krausz, "Power scaling of a high-repetitionrate enhancement cavity,” Opt. Lett. 35(12), 2052-2054 (2010).

9. K. D. Moll, R. J. Jones, and J. Ye, "Output coupling methods for cavity-based high-harmonic generation,” Opt. Express 14(18), 8189-8197 (2006).

10. J. Wu, and H. Zeng, "Cavity-enhanced noncollinear high-harmonic generation for extreme ultraviolet frequency combs," Opt. Lett. 32(22), 3315-3317 (2007).

11. A. Cingöz, D. Yost, J. Ye, A. Ruehl, M. Fermann, and I. Hartl, "Power Scaling of High-Repetition-Rate HHG," International Conference on Ultrafast Phenomena (2010).

12. M. G. Moharam, and T. K. Gaylord, "Rigorous coupled-wave analysis of metallic surface-relief gratings," J. Opt. Soc. Am. A 3(11), 1780-1787 (1986). 
13. M. G. Moharam, E. B. Grann, D. A. Pommet, and T. K. Gaylord, "Formulation for stable and efficient implementation of the rigorous coupled-wave analysis of binary gratings," J. Opt. Soc. Am. A 12(5), 1068-1076 (1995).

14. M. G. Moharam, D. A. Pommet, E. B. Grann, and T. K. Gaylord, "Stable implementation of the rigorous coupled-wave analysis for surface-relief gratings: enhanced transmittance matrix approach,” J. Opt. Soc. Am. A 12(5), 1077-1086 (1995).

15. E. D. Palik, G. Ghosh, and E. J. Prucha, eds., Handbook of Optical Constants of Solids, (Academic Press New York, 1985).

16. M. Schultze, E. Goulielmakis, M. Uiberacker, M. Hofstetter, J. Kim, D. Kim, F. Krausz, and U. Kleineberg, "Powerful 170-attosecond XUV pulses generated with few-cycle laser pulses and broadband multilayer optics," N. J. Phys. 9(7), 243 (2007).

17. T. Eidam, F. Röser, O. Schmidt, J. Limpert, and A. Tünnermann, " 57 W, 27 fs pulses from a fiber laser system using nonlinear compression,” Appl. Phys. B 92(1), 9-12 (2008).

18. T. Hanke, G. Krauss, D. Träutlein, B. Wild, R. Bratschitsch, and A. Leitenstorfer, "Efficient nonlinear light emission of single gold optical antennas driven by few-cycle near-infrared pulses," Phys. Rev. Lett. 103(25), 257404 (2009).

19. I. Pupeza, X. Gu, E. Fill, T. Eidam, J. Limpert, A. Tünnermann, F. Krausz, and T. Udem, "Highly sensitive dispersion measurement of a high-power passive optical resonator using spatial-spectral interferometry," Opt. Express 18(25), 26184-26195 (2010).

20. J. Kaster, I. Pupeza, T. Eidam, C. Jocher, E. Fill, J. Limpert, R. Holzwarth, B. Bernhardt, T. Udem, T. W. Hänsch, A. Tünnermann, and F. Krausz, "Towards MW Average Powers in Ultrafast High-Repetition-Rate Enhancement Cavities," HILAS 2011, Istanbul.

\section{Introduction}

A well-established technique for the production of attosecond XUV light pulses is high harmonic generation (HHG) in noble gases [1]. In order to utilize the HHG process for frequency conversion from the infrared to the XUV wavelength region, a sufficiently high peak intensity has to be obtained. In conventional HHG experiments amplifiers that boost the pulse energy by sacrificing repetition rate have been widely used [2]. Due to the low repetition rate, such XUV sources have a limited range of applications. In particular for experiments, where a combination of a high XUV flux with low energy/pulse is needed in order to e.g. avoid space charge effects, high repetition rate XUV sources are desired. A prominent example is photoelectron emission microscopy (PEEM), which could be used for exploring ultrafast dynamics in nanostructured surface systems with time resolutions down to the attosecond regime [3]. The realization of MHz XUV sources is, however, a challenging task since high driving pulse energies and peak intensities at these repetition rates are needed for the highly-nonlinear HHG process.

The method of cavity-assisted HHG overcomes these limitations. Here, broadband femtosecond pulses from a mode-locked $\mathrm{MHz}$ oscillator are coupled into an external resonant cavity [4-8], in which peak intensities are reached, sufficient to drive intra-cavity HHG at the full repetition rate of the oscillator.

So far, one of the major challenges in cavity-assisted HHG is to efficiently extract the generated high harmonics from the enhancement cavity without influencing the cavity performance. Because intra-cavity generated XUV radiation propagates along the direction of the fundamental beam, it will be blocked and absorbed by the cavity mirrors, which are only highly-reflective for the propagating fundamental light. Thus, an optical element allowing for the output coupling of the intra-cavity generated XUV light is needed, which has high efficiency while exhibiting minimal losses for the fundamental light and also in any other way does not influence the cavity performance.

Several XUV output couplers have been proposed. The first enhancement cavity based HHG systems employed a thin intra-cavity sapphire plate at Brewster's angle for the infrared, while reflecting the XUV radiation [4-6] away from the fundamental radiation beam path. This method nevertheless introduces significant group-velocity dispersion and nonlinear phase shifts at high intensities. To overcome this problem, other methods were proposed, using a small hole in the curved mirror after the intra-cavity focus, where the XUV is generated, or employing a slotted mirror and two colliding pulses to produce non-collinear HHG $[9,10]$. 
However, these methods suffer from increasing additional intra-cavity diffraction losses for the fundamental light and a complex implementation, respectively.

As an alternative to the above mentioned methods, Yost and associates [7] reported a small-period relief diffraction grating for XUV light as an output coupler, which is etched directly into the surface of one of the dielectric mirrors of the cavity. This method overcame the major difficulties by adding minimal intra-cavity loss, and by permitting large buildup peak intensities without introducing nonlinear phase shifts to the cavity resonance. To this day, among the intra-cavity generated XUV output coupling techniques this method is the most promising one from the point of view of power scalability [11]. The maximum theoretical output coupling efficiency at a wavelength of $65 \mathrm{~nm}$, for which the grating design was optimized, amounted to roughly $10 \%$.

In this paper, we report on the design, fabrication and characterization of a nano-period grating, which, in contrast to the one presented in [7] is blazed in order to increase the diffraction and thus output coupling efficiency. The blazed grating was designed such that it serves as a diffractive element for the XUV and acts as high-reflective mirror for the fundamental light. We have theoretically studied and optimized the design of such a nanoperiodical grating to increase the XUV diffraction efficiency to above $20 \%$. Moreover, we characterized the grating using a XUV light source within the wavelength range of 35.5-79.2 $\mathrm{nm}$ and verified its high diffraction efficiency in our experiment. In addition, the influence of the grating structure on the reflectivity of the fundamental IR wavelength and nonlinear effects owed to the nanograting were measured.

\section{Optimization of the grating}

We first compare theoretically two diffraction grating designs (see Fig. 1), one of which is a relief grating based on the one used by Yost et al. [7], the other one is a blazed nanograting, which can lead to higher diffraction efficiency. To determine the optimum design parameters and evaluate the diffraction efficiencies of both gratings, we apply a rigorous coupled-wave analysis method (RCWA) [12-14]. It allows for an arbitrary complex permittivity to be used for the material and thus avoids the infinite conductivity approximation. The wavelength dependent dielectric constants used in these calculations are based on experimental data [15].

The goal of our optimization was to obtain high coupling efficiency between 35.5 and 79.2 $\mathrm{nm}$, corresponding to the 13th to 29th harmonics of a fundamental wavelength of $1030 \mathrm{~nm}$. One motivation for the optimization of the efficiency in this wavelength range comes from the possibility to conduct high resolution spectroscopy of the $1 \mathrm{~s}-2 \mathrm{~s}$ transition in $\mathrm{He}^{+}$at around 61 $\mathrm{nm}$ with extreme precision [4,6]. It should be stressed that the choice of the IR wavelength range for the design can be easily varied.

The nano-period grating structure is implemented on a multi-layer dielectric mirror, which is designed as a near-perfect reflector for the fundamental wavelength of $1030 \mathrm{~nm}$ with spolarization. The grating is structured into the uppermost $\mathrm{SiO}_{2}$ layer of the multi-layer mirror.

The optimization parameters include the incidence angle, groove period, groove depth, the duty cycle, and blaze angle. In Fig. 1c we compare the optimized diffraction efficiencies in the -1 st order (higher orders are less efficient) within the given wavelength range for a relief and a blazed nanograting. Note that the fundamental light is only present in the 0th order of diffraction, such that for a structure with optimized -1 st diffraction order for the XUV, these two wavelength ranges can be easily separated. 
(a)

(b)

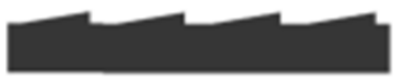

(c)

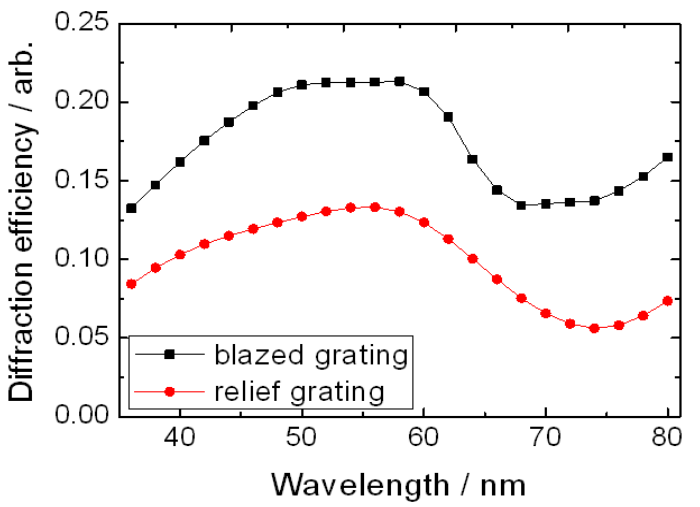

Fig. 1. Comparison of the diffraction efficiency of a relief nanograting (a) and a blazed nanograting (b). Theoretically, the blazed grating can achieve an efficiency of around $20 \%$, which is considerably higher than for a relief grating (around 10\%), (c).

The parameters for an optimized relief grating (incidence angle: 70 degrees, grove period: $420 \mathrm{~nm}$, groove depth: $40 \mathrm{~nm}$, duty cycle: $40 \%$ ) based on the RCWA method exhibit only small differences to the nanograting used by Yost et al. giving a diffraction efficiency of about $10 \%$ over the studied wavelength range. For the blazed grating the optimized parameters were identified for an incidence angle of 72 degrees, $510 \mathrm{~nm}$ groove period, $46 \mathrm{~nm}$ groove depth, $70 \%$ duty cycle, and a blaze angle of 3.6 degrees. The results of the theoretical simulations for this blazed grating are compared to the relief grating in Fig. 1c. The blazed grating could achieve an efficiency of up to about $20 \%$, which is considerably higher than for the relief grating with approx. 10\%. Between the 17th and 21 st harmonics $(50 \mathrm{~nm}$ to $60 \mathrm{~nm})$, the blazed nanograting is found to provide a diffraction efficiency in the -1 st order even exceeding $20 \%$.

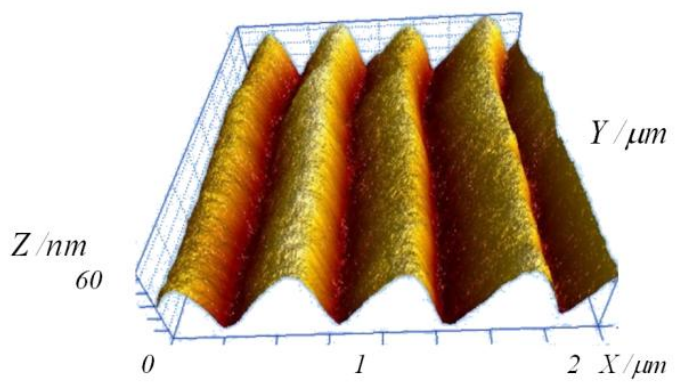

Fig. 2. AFM image of the nanostructured blazed grating. The grating is etched into the uppermost $\mathrm{SiO}_{2}$ layer. The groove period is $510 \mathrm{~nm}$; groove depth is $60 \mathrm{~nm}$ and aspect ratio is $70 \%$.

To fabricate the grating, we used the dielectric mirror as the substrate, which is an IR nearperfect reflector (Layertec), R > 99.95\% at 1000-1040 nm. Firstly, the substrate was coated with the electron beam resist ARP 617.03. The blazed resist profile was created with the electron beam lithography system Vistec SB350 using a variable dose approach. After the development of the resist mask, it was proportionally transferred into the substrate by ICPRIE (inductive coupled plasma-reactive ion etching). We employed $\mathrm{CHF}_{3}$ as process gas and O2-ICP-RIE to remove all organic residues after this transfer. On account of limitations in the manufacturing process, the blazed grating structure had a groove depth of $60 \mathrm{~nm}$. Figure 2 depicts an atomic force microscopy (AFM) image of the produced blazed nanograting. 


\section{XUV diffraction efficiency measurements}

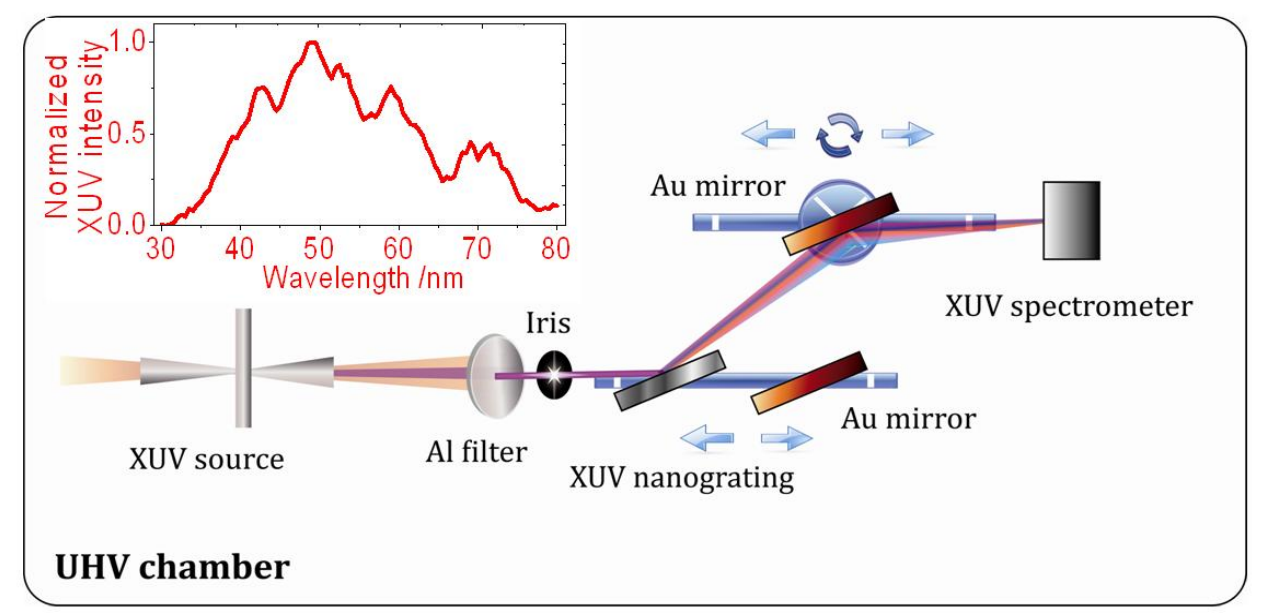

Fig. 3. Illustration of the experimental setup for the diffraction efficiency measurements. The entire setup is placed in a vacuum chamber. The broadband XUV radiation (see inset) is generated by a few-cycle phase-stabilized Ti:Sa laser pulse $(750 \mathrm{~nm}, 4 \mathrm{fs})$ via HHG in a Xe gas jet. An aluminium filter allows transmission of XUV light below $79.2 \mathrm{~nm}$ while removing the residual IR light. The XUV is either diffracted by the blazed nanograting or reflected by a flat gold mirror and then steered into an XUV spectrometer by another gold mirror. In order to compensate for the angular dispersion of the nanograting the second gold mirror could be translated and rotated.

To experimentally characterize the performance of the blazed nanograting, the setup sketched in Fig. 3 was used. Briefly, few-cycle IR laser pulses $(750 \mathrm{~nm}, 4 \mathrm{fs})$ were used to generate a broadband XUV spectrum by HHG [16] within a Xe gas jet at peak intensities exceeding $10^{13}$ $\mathrm{W} / \mathrm{cm}^{2}$ in the focus. The gas target is contained within a nickel tube with an inner diameter of $3 \mathrm{~mm}$. Both XUV and IR laser pulses propagate collinearly after the gas target. A $200 \mathrm{~nm}$ thick $\mathrm{Al}$ filter is used to remove the fundamental laser beam and low order harmonics. An iris with a diameter of $3 \mathrm{~mm}$ was placed after the $\mathrm{Al}$ filter to ensure that only the nanograting (measuring $4 \times 10 \mathrm{~mm}$ ) etched into the top of the dielectric mirror was illuminated by the XUV beam (rather than the surface of dielectric mirror). After either diffraction from the nanograting or reflection from a flat gold mirror, the XUV light is imaged into a scanning XUV monochromator for the setup described here. The angular dispersion of the nanograting was compensated for by adjusting the rotation and translation of the imaging gold mirror (see Fig. 3 for specific wavelengths). The entire setup is placed in a vacuum chamber and evacuated to a pressure of $10^{-5}$ mbar. Absorption of XUV light by residual gas in the chamber can thus be neglected. Our setup provided the possibility to measure the diffraction efficiency at any particular wavelength within the broad spectral range of the XUV source.

In order to independently calibrate the XUV intensity for each wavelength, we introduced a gold mirror into the beam instead of the nanograting as a reference. Both the grating and the XUV reflection mirror are situated on a translation stage. In order to avoid systematic drifts in the XUV spectrum from the HHG process (due to intensity fluctuations of the laser) to influence the measurement, alternating measurements were performed for the XUV intensity of the 0th diffraction order of the grating and the reflection from the mirror. In this way, the ratio $R_{0 \text { th/mirror }}$ of the 0 th diffraction order efficiency of the grating and the reflectivity of the mirror was determined (see black line in Fig. 4a). In order to measure the -1 st order diffraction efficiency of the nanograting, the ratio $\mathrm{R}_{-1 \mathrm{st} / \mathrm{th}}$ of the -1 st and 0 th order diffraction efficiencies was characterized. Note that $\mathrm{R}_{-1 \mathrm{st} / 0 \text { th }}$ was corrected for the reflectivity of the second gold mirror for different incidence angles. The reflectivity of the gold mirror is derived from the refraction indices given in Palik's handbook [15]. 

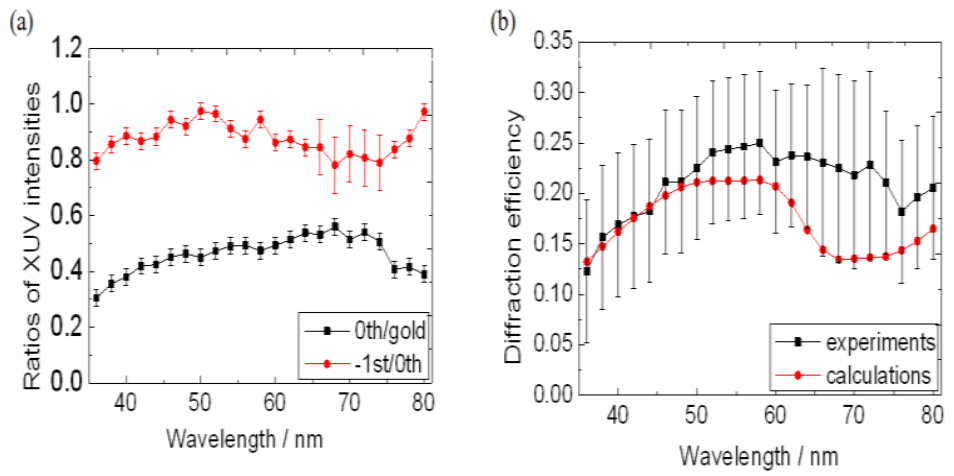

Fig. 4. (a) The measured ratios of XUV intensities. $R_{0 \text { th/gold }}$ is the ratio of 0th order efficiency of the grating and gold mirror. The error bars given for the black curve stem from drifts in the XUV spectrum and are determined as $0.03 . R_{-1 \text { st } / 0 \text { th }}$ is the ratio of -1 st and 0th order efficiency of the grating. The error bars given for the red curve are statistical and correspond to the standard deviation $(\sigma)$. (b) The calculated -1 st order efficiency (red curve) and the extracted -1 st order efficiency (black curve) from experiments. The error bars are based on the ones in (a) and they take into account systematic errors due to the calibration of the reflectivity by using the gold mirror. These errors are determined as 0.09 in the range from $66 \mathrm{~nm}$ to $74 \mathrm{~nm}$ and 0.07 at other wavelengths.

The output coupling efficiency of the -1 st order, Eff -1st $_{\text {, was }}$ extracted from the described measurements as follows:

$$
\operatorname{Eff}_{-1 \text { st }}(\lambda)=\mathrm{R}_{-1 \mathrm{st} / 0 \text { th }}(\lambda) \times \mathrm{R}_{0 \text { th } / \text { gold }}(\lambda) \times \operatorname{ref}_{\text {gold }}(\lambda)
$$

where ref $_{\text {gold }}$ is the gold reflectivity for different wavelengths. The result is displayed in Fig. 4b. In order to account for the limited resolution in the experimental measurements, the theoretical prediction that is displayed as red line in Fig. 4b has been convoluted with a Gaussian of $4 \mathrm{~nm}$ full-width at half-maximum. The experimental result is in reasonable agreement with the theoretical prediction. Notably, the experimental measurement around 70 $\mathrm{nm}$ gives a higher diffraction efficiency than theoretically predicted. At around $70 \mathrm{~nm}$ the refractive index of $\mathrm{SiO}_{2}$ goes to 1, which leads to a dip in the -1st order diffraction. Although the dip is also evident in the measurement it is not as pronounced as in the theory. There are two potential reasons which could cause the deviations between the theoretical and experimental data. One is the roughness due to the fabrication limits of nanograting; the other is a possible change in the stoichiometric composition of the $\mathrm{SiO}_{2}$ layer in the production process, which could affect the reflectivity of the dielectric surface of the grating in the XUV range.

To our best knowledge, this is the first full characterization of the -1 st order XUV diffraction efficiency of a blazed nanograting. The fabricated dielectric nanograting exhibits a diffraction efficiency of more than $20 \%$ between $50 \mathrm{~nm}$ and $60 \mathrm{~nm}$. Using such a blazed nanograting as output coupler in an enhancement cavity would result in high external XUV powers.

\section{IR reflectivity and high-power nonlinearity of the blazed nanograting}

In order to characterize the IR reflectivity of the nanograting, we used an optical lossmeter (LossPro, central wavelength 1030nm). Here, the mirror under test is implemented as one of the mirrors of a non-resonant cavity. A pulse is coupled to the cavity and the exponential decay of its intensity upon many bounces on the cavity mirrors is recorded. The total losses of the investigated mirror are calculated from a fit to the exponential decay, while the losses of the other cavity mirrors are known from a separate calibration measurement. To enable a continuous variation of the incidence angle on the investigated mirror, we have modified the 
lossmeter cavity while keeping its length constant (which is crucial for an accurate measurement). We calibrated the modified cavity at the incidence angles for which the original device was designed, thus ensuring the reliability of our measurements. We compared the losses introduced by the nanograting to the losses of the original $\mathrm{SiO}_{2}$ substrate of the dielectric mirror for different incidence angles (see Fig. 5). The reflectivity of the dielectric mirror is found to be larger than $99.994 \%$. For the blazed grating, the IR reflectivity still remained large with more than $99.97 \%$.

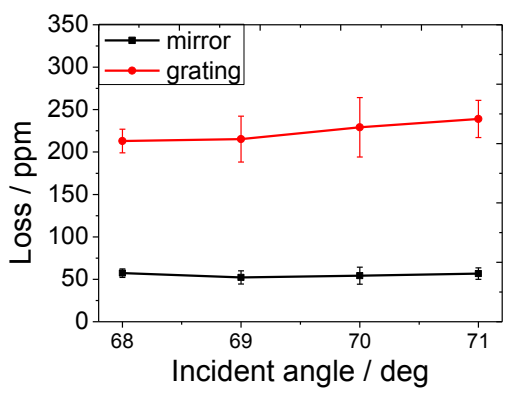

Fig. 5. The measured losses of the nanograting and dielectric mirror at the fundamental wavelength. The average of the loss of the grating is lower than $300 \mathrm{ppm}$ (corresponding to a reflectivity $>99.97 \%$ ), while the dielectric mirror without grating has a loss lower than $60 \mathrm{ppm}$ (reflectivity $>99.994 \%$ ). The error bars are statistical and correspond to the standard deviation $(\sigma)$.

In order to investigate nonlinear effects at the nanograting, we used a z-scan setup shown in Fig. 6 and compared the properties of the nanograting with the dielectric mirror without grating.

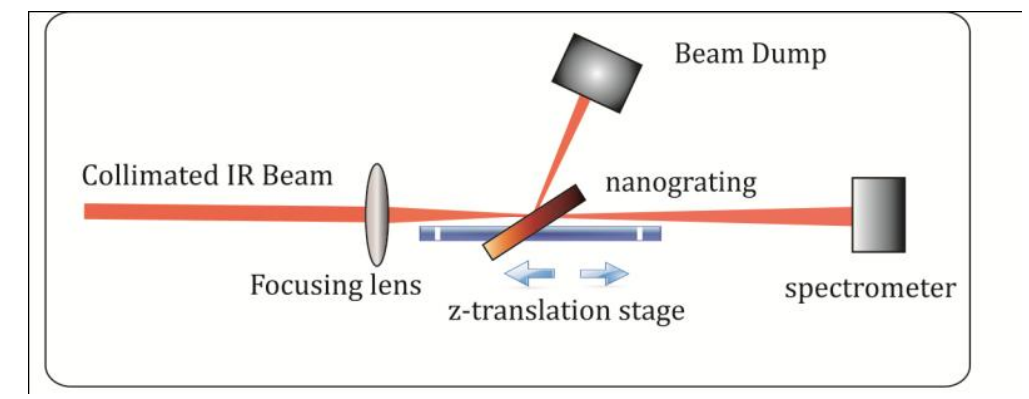

Fig. 6. Z-scan experimental setup: the nanograting is scanned under a $60^{\circ}$ angle of incidence through the focused infrared laser beam with $1040 \mathrm{~nm}$ central wavelength, $40 \mathrm{~W}$ average power, $78 \mathrm{MHz}$ repetition rate and $200 \mathrm{fs}$ pulse duration. The signal transmitted through the mirror is monitored with an UV spectrometer. The focus of focusing lens is $150 \mathrm{~mm}$, focus diameter is $55 \mu \mathrm{m}$, and collimated IR beam diameter is $3.6 \mathrm{~mm}$.

We varied the intensity of an infrared laser beam impinging on the surface of the mirror with the nanograting structure by scanning it through a focus and monitored the third harmonic $(\mathrm{TH})$ generated at the optical element and transmitted through it. The $3.6 \mathrm{~mm}$ diameter collimated output beam of the CPA system [17] is focused with a lens of an effective focal length of $150 \mathrm{~mm}$ down to $55 \mu \mathrm{m}$ diameter. The laser parameters used here are: 1040 $\mathrm{nm}$ central wavelength, $40 \mathrm{~W}$ of average power, $78 \mathrm{MHz}$ repetition rate and $200 \mathrm{fs}$ pulse duration. A reproducible intensity variation is achieved by scanning the mirror along the optical axis (z-scan) through the focus. The angle of incidence on the mirror equals $60^{\circ}$, which is a geometrical constraint of our z-scan setup. The transmitted portion of the beam is sent to an UV spectrometer (Ocean Optics HR4000). 


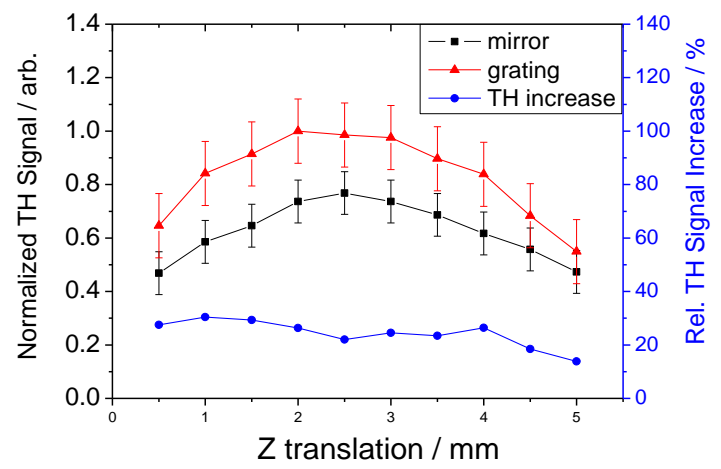

Fig. 7. Z-scan measurement results. The measurement gives insight into the optical properties of the high power nonlinearity introduced by the nanograting: the increase of the third harmonic intensities generated at the nanograting and at the plane surface of the same dielectric mirror is around $30 \%$. The error bars for the red and black curves are statistical and correspond to the standard deviation $(\sigma)$.

We investigated two cases: (i) the grating and (ii) a plane area on the same dielectric mirror surface was irradiated. For both cases we recorded the intensity of the TH (spectrometer counts at the third harmonic spectrum maximum), centered at $347 \mathrm{~nm}$, as a function of the $\mathrm{z}$ position. The measurement for (i) has an absolute error of 0.12 in our measurement, and (ii) has an error of 0.08. Figure 7 shows the results. An enhancement of the $\mathrm{TH}$ is clearly visible in case of the nanograting. In comparison to the TH intensity generated at the dielectric mirror without grating, the TH intensity generated at the grating is increased by about $30 \%$. Similar to the observed THG enhancement by the nanostructure on a dielectric substrate, THG enhancement was observed for metallic nanostructures attached to a dielectric, see e.g [18]. A detailed investigation of the physical mechanism behind this effect is the subject of future work.

This effect is likely to limit the maximum storable peak and / or average power as well as the minimum duration of the circulating pulse inside an enhancement cavity including the nanograting. On the one hand, with increasing intensities, THG introduces significant losses to the circulating field amplitude, affecting the enhancement. On the other hand, the THG, which is also observed from the plane mirror surface, might be correlated to an intensitydependent variation of the nonlinear group velocity dispersion of the IR mirror. This effect has been observed in [19] for highly reflective mirrors. Despite these limitations, impressive values of $5 \mathrm{~kW}$ of circulating average power at a pulse duration of $120 \mathrm{fs}$ and a repetition rate of $154 \mathrm{MHz}$ were achieved in a cavity incorporating a nanograting recently [11]. Further scaling could be achieved with the approach of increasing the IR beam size on the nanograting, for instance using the cavity design presented in [20]. Moreover, not only the reflectivity of the optical element for the IR radiation, but also its diffraction efficiency in the XUV range could be altered in the high-power regime. These effects are subject to further investigation.

\section{Conclusion}

To conclude, we have designed, fabricated and characterized a blazed nanograting, which may serve as efficient output coupler for XUV radiation generated in a femtosecond enhancement cavity. A very high -1 st order diffraction efficiency around $20 \%$ for the wavelength range from $35.5 \mathrm{~nm}$ to $79.2 \mathrm{~nm}$ was measured for the blazed nanograting. In addition to the XUV diffraction efficiency, we have measured the IR reflectivity and high-power non-linearities of such an optical element. 


\section{Acknowledgments}

We acknowledge experimental support by Tino Eidam from the IAP, University of Jena. This work was supported by the BMBF under PhoNa - Photonische Nanomaterialien, contract number 03IS2101B, the DFG via the Emmy-Noether program and SPP1391. Y.-Y. Y. acknowledges support by a Chinese Academy of Sciences fellowship and S.L.S. acknowledges support by a fellowship of the Alexander von Humboldt foundation. 


\title{
Transverse mode tailoring in a quasi-imaging high-finesse femtosecond enhancement cavity
}

\author{
Johannes Weitenberg, ${ }^{1, *}$ Peter Rußbüldt, ${ }^{2}$ Tino Eidam, ${ }^{3}$ and Ioachim Pupeza ${ }^{4}$ \\ ${ }^{I}$ Lehrstuhl für Lasertechnik LLT, RWTH Aachen University, Steinbachstr. 15, 52074 Aachen, Germany \\ ${ }^{2}$ Fraunhofer-Institut für Lasertechnik ILT, Steinbachstr. 15, 52074 Aachen, Germany \\ ${ }^{3}$ Friedrich-Schiller-University Jena, Institute of Applied Physics, Albert-Einstein-Str. 15, 07745 Jena, Germany \\ ${ }^{4}$ Max-Planck-Institut für Quantenoptik MPQ, Hans-Kopfermann-Str. 1, 85748 Garching, Germany \\ *johannes.weitenberg@ilt.fraunhofer.de
}

\begin{abstract}
We demonstrate a high-finesse femtosecond enhancement cavity with an on-axis obstacle. By inserting a wire with a width of 5\% of the fundamental mode diameter, the finesse of $\mathrm{F}=3400$ is only slightly reduced to $\mathrm{F}=3000$. The low loss is due to the degeneracy of transverse modes, which allows for exciting a circulating field distribution avoiding the obstacle. We call this condition quasi-imaging. The concept could be used for output coupling of intracavity-generated higher-order harmonics through an onaxis opening in one of the cavity mirrors.
\end{abstract}

(C)2011 Optical Society of America

OCIS codes: (070.5753) Resonators; (140.7240) UV, EUV, and X-ray lasers; (190.4160) Multiharmonic generation; (320.7110) Ultrafast nonlinear optics.

\section{References and links}

1. I. Pupeza, T. Eidam, J. Rauschenberger, B. Bernhardt, A. Ozawa, E. Fill, A. Apolonski, Th. Udem, J. Limpert, Z. A. Alahmed, A. M. Azzeer, A. Tünnermann, T. W. Hänsch, and F. Krausz, "Power scaling of a high-repetitionrate enhancement cavity,” Opt. Lett. 35(12), 2052-2054 (2010).

2. A. Ozawa, J. Rauschenberger, Ch. Gohle, M. Herrmann, D. R. Walker, V. Pervak, A. Fernandez, R. Graf, A. Apolonski, R. Holzwarth, F. Krausz, T. W. Hänsch, and Th. Udem, "High harmonic frequency combs for high resolution spectroscopy," Phys. Rev. Lett. 100(25), 253901 (2008).

3. I. Pupeza, T. Eidam, J. Kaster, B. Bernhardt, J. Rauschenberger, A. Ozawa, E. Fill, Th. Udem, M. F. Kling, J. Limpert, Z. A. Alahmed, A. M. Azzeer, A. Tünnermann, Th. W. Hänsch, and F. Krausz, "Power scaling of femtosecond enhancement cavities and high-power applications," Proc. SPIE 7914, 79141I, 79141I-13 (2011).

4. R. J. Jones, K. D. Moll, M. J. Thorpe, and J. Ye, "Phase-coherent frequency combs in the vacuum ultraviolet via high-harmonic generation inside a femtosecond enhancement cavity," Phys. Rev. Lett. 94(19), 193201 (2005).

5. Ch. Gohle, Th. Udem, M. Herrmann, J. Rauschenberger, R. Holzwarth, H. A. Schuessler, F. Krausz, and T. W. Hänsch, "A frequency comb in the extreme ultraviolet," Nature 436(7048), 234-237 (2005).

6. D. C. Yost, T. R. Schibli, and J. Ye, "Efficient output coupling of intracavity high-harmonic generation," Opt. Lett. 33(10), 1099-1101 (2008).

7. Y.-Y. Yang, F. Süßmann, S. Zherebtsov, I. Pupeza, J. Kaster, D. Lehr, H.-J. Fuchs, E.-B. Kley, E. Fill, X.-M. Duan, Z.-S. Zhao, F. Krausz, S. L. Stebbings, and M. F. Kling, "Optimization and characterization of a highlyefficient diffraction nanograting for MHz XUV pulses," Opt. Express 19(3), 1954-1962 (2011).

8. K. D. Moll, R. J. Jones, and J. Ye, "Output coupling methods for cavity-based high-harmonic generation," Opt. Express 14(18), 8189-8197 (2006).

9. W. P. Putnam, G. Abram, E. L. Falcão-Filho, J. R. Birge, and F. X. Kärtner, "High-intensity Bessel-Gauss beam enhancement cavities," in Conference on Lasers and Electro-Optics, OSA Technical Digest (CD) (Optical Society of America, 2010), paper CMD1 (2010).

10. A. Ozawa, A. Vernaleken, W. Schneider, I. Gotlibovych, Th. Udem, and T. W. Hänsch, "Non-collinear high harmonic generation: a promising outcoupling method for cavity-assisted XUV generation," Opt. Express 16(9), 6233-6239 (2008).

11. S. Gigan, L. Lopez, N. Treps, A. Maître, and C. Fabre, "Image transmission through a stable paraxial cavity," Phys. Rev. A 72(2), 023804 (2005).

12. R. Paschotta, "Beam quality deterioration of lasers caused by intracavity beam distortions," Opt. Express 14(13), 6069-6074 (2006).

13. J. Weitenberg, P. Rußbüldt, I. Pupeza, Th. Udem, H.-D. Hoffmann, and R. Poprawe, "Geometrical on-axis access to high-finesse resonators by quasi-imaging," (manuscript in preparation).

14. T. Eidam, F. Röser, O. Schmidt, J. Limpert, and A. Tünnermann, " $57 \mathrm{~W}, 27$ fs pulses from a fiber laser system using nonlinear compression,” Appl. Phys. B 92(1), 9-12 (2008).

15. Th. W. Hänsch and B. Couillaud, "Laser frequency stabilization by polarization spectroscopy of a reflecting reference cavity," Opt. Commun. 35(3), 441-444 (1980). 
16. T. C. Briles, D. C. Yost, A. Cingöz, J. Ye, and T. R. Schibli, "Simple piezoelectric-actuated mirror with $180 \mathrm{kHz}$ servo bandwidth," Opt. Express 18(10), 9739-9746 (2010).

17. J. A. Arnaud, "Degenerate optical cavities," Appl. Opt. 8(1), 189-195 (1969).

18. D. Esser, W. Bröring, J. Weitenberg, and H.-D. Hoffmann, "Laser-manufactured mirrors for geometrical output coupling of intracavity-generated high harmonics," (manuscript in preparation).

19. F. V. Hartemann, W. J. Brown, D. J. Gibson, S. G. Anderson, A. M. Tremaine, P. T. Springer, A. J. Wootton, E. P. Hartouni, and C. P. J. Barty, "High-energy scaling of Compton scattering light sources," Phys. Rev. ST Accel. Beams 8(10), 100702 (2005).

20. R. Grimm, M. Weidemüller, and Y. B. Ovchinnikov, "Optical dipole traps for neutral atoms,” Adv. At. Mol. Opt. Phys. 42, 95-170 (2000).

\section{Introduction}

The generation of high harmonics $(\mathrm{HHG})$ of femtosecond radiation in a gas makes possible table-top coherent light sources with wavelengths down to a few nanometers. To reach the required peak intensities on the order of $10^{14} \mathrm{~W} / \mathrm{cm}^{2}$ for the nonlinear conversion at $\mathrm{MHz}$ repetition rates, as well as to increase the yield of the highly inefficient conversion process, resonant enhancement in a passive cavity can be employed [1-10]. In the infrared range, a power enhancement of three orders of magnitude has been demonstrated [1]. High repetition rates (several tens of $\mathrm{MHz}$ and more) are desirable for several applications, including high precision spectroscopy in the XUV range [2] and coincidence spectroscopy of correlated electron dynamics [3]. The intracavity-generated high harmonics propagate along the resonator optical axis. Their output coupling from the cavity is a major challenge and has been subject to intensive research over the past few years [6-10]. An established method relies on a thin plate, placed behind the focus at Brewster's angle for the fundamental radiation [2,4,5]. The intracavity-generated XUV radiation experiences total reflexion at the plate and is thus separated from the fundamental beam. However, due to strong absorption of the evanescent field, the output coupling efficiency is typically $0.1-0.2$ for wavelengths $\sim 60 \mathrm{~nm}$ and significantly decreases for shorter wavelengths. For wavelengths around $10 \mathrm{~nm}$ this method is not suitable because the efficiency is typically $<10^{-3}$. Moreover, nonlinearities introduced by the plate limit the peak power circulating in the cavity. A different output coupling method uses a grating, manufactured in the top coating layer of a cavity mirror behind the focus [6,7]. While the XUV radiation is diffracted by the grating, the optical element acts as a highly reflecting mirror for the fundamental beam. The output coupling efficiency is typically $<0.2$ and also decreases for smaller wavelengths. Recently, it has been shown that the nanostructure leads to increased nonlinear effects on the mirror surface induced by the fundamental radiation [7], which might limit the scaling of intracavity peak power. Moreover, the fact that the harmonics are spatially dispersed might constitute a drawback for some applications. In a third approach, the output coupling is achieved geometrically through an on-axis hole or slit in the mirror behind the focus. The smaller divergence angle of the high harmonics due to the shorter wavelength makes this approach possible. Because there is no interaction of the XUV radiation with any optical element, a geometrical access allows for efficient output coupling of short wavelengths (e.g. sub-10 nm). Moreover, no dispersion or nonlinearities are introduced by the output coupling mechanism. If the fundamental mode of the cavity is used, an on-axis hole has to be very small to allow for a high enhancement [8]. Approaches allowing for a broad hole or slit at small loss for the fundamental radiation have been proposed by employing either transverse modes with vanishing field on the optical axis [8], an imaging resonator [9] or by using non-collinear high harmonic generation $[8,10]$.

In this paper we experimentally demonstrate the possibility of geometrical on-axis access to a high-finesse enhancement cavity by a technique which we call quasi-imaging. By particular choice of the resonator geometry, higher-order transverse modes can be simultaneously resonant with the fundamental mode. In our case, the Gauss-Hermite modes $G_{0,0}, \mathrm{GH}_{4,0}$ and $\mathrm{GH}_{8,0}$ can be excited simultaneously. If an obstacle, e.g. a wire or a slit in a resonator mirror, is introduced into the beam path, these modes arrange to avoid the obstacle. We call this quasi-imaging, because only a subset of the transverse modes is involved, whereas in an imaging resonator all transverse modes are simultaneously resonant. 
The manuscript is structured as follows: Section 2 contains an introduction to the concept of quasi-imaging, in Section 3 we present the experimental realization of a quasi-imaging resonator, Section 4 gives a brief discussion and Section 5 concludes the paper.

\section{Quasi-imaging}

\subsection{Definition of a quasi-imaging resonator}

For a stable spherical resonator in paraxial approximation, one set of eigen-modes, i.e. field distributions which are reproduced after one resonator round trip, are the Gauss-Hermite $G H_{n, m}$ modes. When an obstacle is placed in the beam path, these modes are in general no longer the resonator eigen-modes. However, if a set of $G H$ modes is simultaneously resonant, new eigen-modes can be constructed as a combination thereof, which avoid the obstacle and have small diffraction loss. We call this condition quasi-imaging. Quasi-imaging is related to imaging, because the hole in a field distribution is reproduced after a resonator round trip. However, quasi-imaging can be achieved in a stable resonator (i.e. with a distinguished eigenq-parameter, or equivalently for $|A+D|<2$ with the elements of the beam transfer matrix for one resonator round trip $M=[[A, B],[C, D]])$, which is not the case for imaging. The on-axis phase $\phi$ acquired at a resonator round trip by a $G H_{n, m}$ mode with eigen-q-parameter ( $q_{x}$ and $\left.q_{y}\right)$ and wave number $k$ is given by

$$
\phi_{k, n, m}=k L+\left(n+\frac{1}{2}\right) \psi_{x}+\left(m+\frac{1}{2}\right) \psi_{y}
$$

where $L$ is the resonator length (length of a ring resonator or twice the length of a linear resonator) and $\psi_{x, y}$ is a phase which we will refer to as the Gouy parameter. This phase is due to the Gouy effect and is defined by the resonator geometry [11]. The Gouy parameter and the eigen-q-parameter can be different for the two transverse directions in Cartesian geometry. The stability range of the resonator is determined by the condition $0<\psi<\pi$ or $\pi<\psi<2 \pi$. Modes with mode number difference $\Delta n$ are simultaneously resonant if the Gouy parameter is $\psi_{x}=2 \pi h / \Delta n$ with an integer $h$, because their round-trip phases are equal $\bmod (2 \pi)$. The simplest case, in which a zero of the electric field at the position of the obstacle is achieved, is the combination of the fundamental mode with the next resonant transverse mode. In the middle of the stability range, where the Gouy parameter is $\psi=\pi / 2$ or $3 \pi / 2$, the mode number difference for simultaneously resonant modes is $\Delta n=4$. The corresponding mode combination is shown in Fig. 1. It constitutes a new eigen-mode of the resonator with a sufficiently small obstacle on the optical axis. The obstacle leads to a coupling between the modes [12].

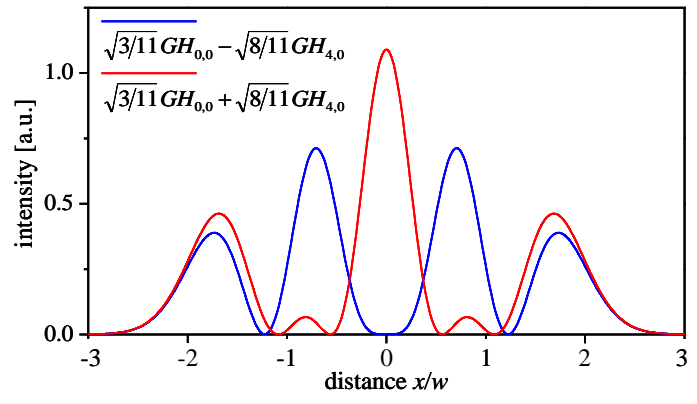

Fig. 1. Eigen-mode of a quasi-imaging resonator at the position of the obstacle and at the position of maximum intensity on the optical axis in Cartesian geometry with mode number difference $\Delta n=4$. To achieve zero on the optical axis, the modes have to be added with the indicated coefficients and opposite sign. Because the modes acquire a different on-axis phase $\phi$ at propagation, at a different position they are added with equal sign, which yields an on-axis intensity maximum. The spatial overlap with a Gaussian beam with the same q-parameter can be calculated from the coefficients and is $U=3 / 11$. 
Further resonant $G H$ modes can contribute to the field distribution. This can change the spatial overlap with the incident field, the width of the on-axis region of small intensity, and loss at apertures limiting the transverse extent of the field distribution. Such apertures can be used to suppress the contribution of higher-order $G H$ modes.

If a resonator has cylindrical symmetry, the Gouy parameters in the transverse directions are equal, i.e. $\psi_{x}=\psi_{y}$, and the Gauss-Laguerre modes $G L_{p}^{l}$ with radial and azimuth mode index $p$ and $l$ can be used instead of the $G H$ modes as an orthonormal basis of eigen-modes. Various mode number differences and mode combinations can be chosen. A detailed theoretical description exceeds the scope of this paper and will be provided in [13].

\subsection{Geometrical on-axis access to a bow-tie ring resonator by quasi-imaging}

The simplest setup of a ring resonator, as it is e.g. commonly used for HHG, is a resonator with two focusing mirrors with radius of curvature $R_{C}$ and resonator length $L$ (see Fig. 2). The distance $d$ of the focusing mirrors determines the Gouy parameters $\psi_{x}$ and $\psi_{y}$ in the transverse directions. The angle of incidence $\alpha$ on the curved mirrors causes different Gouy parameters in the transverse directions, i.e. $\psi_{x} \neq \psi_{y}$. Therefore, the condition for quasi-imaging can be achieved only in one transverse direction. In the middle of the stability range, for $\psi_{y}=3 \pi / 2$, the modes $\mathrm{GH}_{0,0}$ and $\mathrm{GH}_{0,4}$ are simultaneously resonant and can be combined to a "slit mode" avoiding an on-axis slit in the output coupling mirror. The on-axis intensity oscillates along the propagation direction, with zero on the optical axis in the focal plane and an intensity maximum one Rayleigh length from the focus (see Fig. 2).

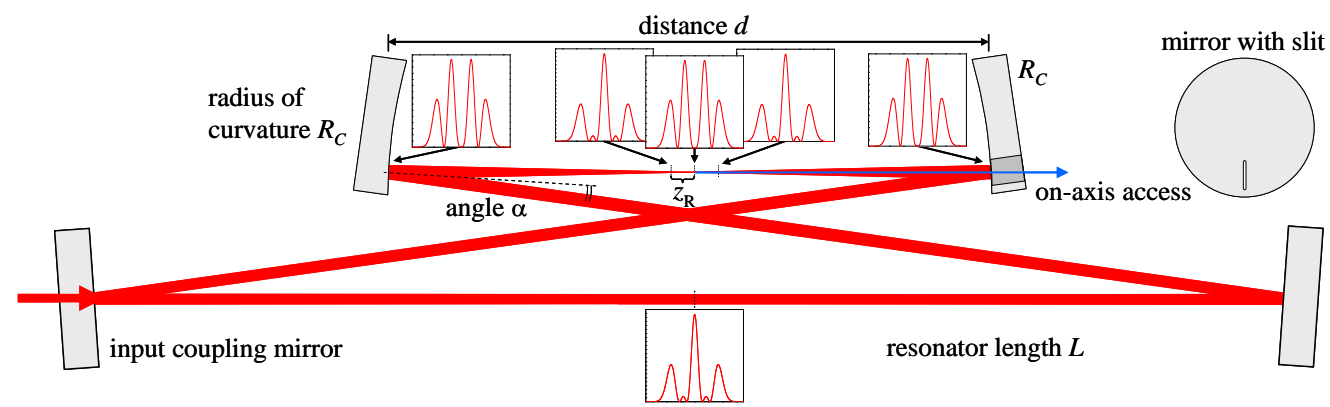

Fig. 2. Quasi-imaging ring resonator. In the middle of the stability range, the Gouy parameter $\psi_{y}$ for one round trip in the sagittal transverse direction (orthogonal to the plane of the beam path) is $\psi_{y}=3 \pi / 2$ and the modes $G H_{0,0}$ and $G H_{0,4}$ are simultaneously resonant. The Gouy parameter acquired in the short arm is $\psi_{y} \sim \pi$ and in the long arm $\psi_{y} \sim \pi / 2$. The intensity distribution in the transverse direction of the quasi-imaging is shown at selected positions. The slit allows for a geometrical access (blue arrow) to the optical axis which could be used for output coupling of high harmonics generated in a gas jet near the focus.

\subsection{Adjustment of transverse mode degeneracy}

Quasi-imaging relies on a degeneracy of transverse modes, which are simultaneously excited to form a field distribution avoiding an obstacle. A degeneracy can be easily adjusted by interpreting the scan pattern, i.e. the transverse mode resonances at a scan of the oscillator cavity length, for the enhancement cavity without an obstacle. Figure 3 shows a schematic diagram of such a scan pattern for a femtosecond bow-tie ring resonator. The abscissa shows the varied oscillator cavity length multiplied with the wave number and the ordinate shows the corresponding intracavity power. Resonances of the fundamental mode $\left(\mathrm{GH}_{0,0}\right)$ appear with a period of $2 \pi$, corresponding to a change in cavity length by one wavelength $\lambda$. Because higher-order transverse modes acquire an additional phase (Eq. (1)), they are resonant at smaller frequencies than the fundamental mode, i.e. at longer oscillator cavity lengths. As can be easily seen, the Gouy parameter in tangential direction $\psi_{x}$ is larger than in sagittal direction $\psi_{y}$ for a bow-tie ring resonator. This difference between the Gouy parameters becomes visible in a resonator with finesse $F$, if the resonance width $2 \pi / F$ is smaller than the Gouy parameter dif- 
ference $\psi_{x}-\psi_{y}$. The position in the stability range can be read from the position of the resonances, the Gouy parameter being the distance between the 0-resonance and 1-resonance. In practice, the transverse modes can be easily identified by the number of adjacent resonances and by tilting of the incident beam and thereby changing its spatial overlap with the cavity transverse modes. Mode $G_{4,0}$ acquires an additional phase compared to the fundamental mode, which is four times the Gouy parameter $\psi_{x}$. Therefore, the two modes are simultaneously resonant if $\psi_{x}=3 \pi / 2$ holds. In the situation shown in Fig. 3, $\psi_{x}$ has to be increased (by increasing the distance $d$ between the curved mirrors) in order to achieve the degeneracy.

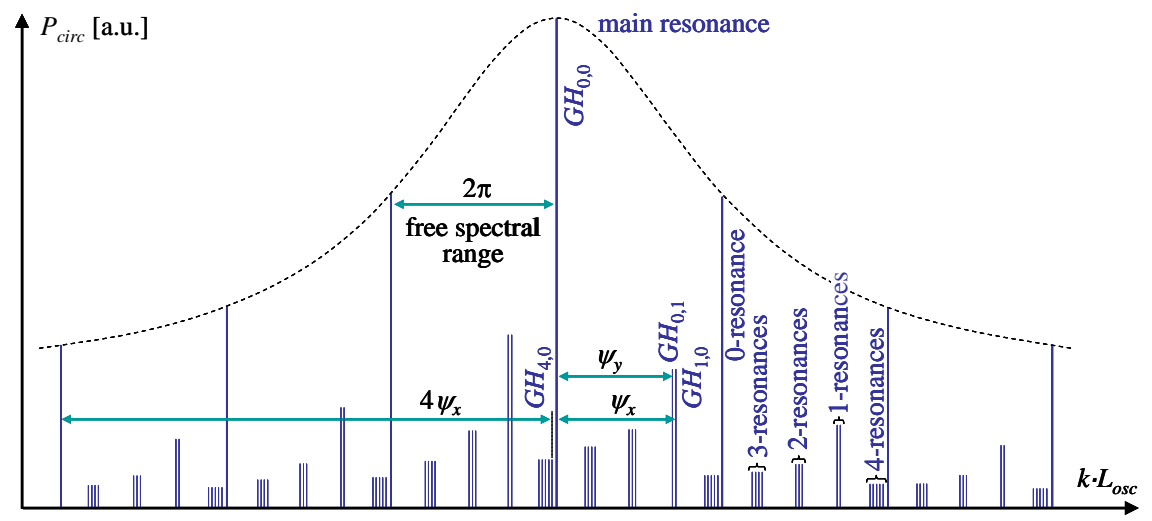

Fig. 3. Schematic diagram of the resonances for a scan of the oscillator cavity length $L_{o s c}$. $k$ is the wave number corresponding to the central wavelength of the incident femtosecond pulse train. The relative height of the resonances depends on the spatial overlap with the incident beam and is arbitrarily chosen. Resonances of higher order than 4 are not shown. To a group of resonances with constant sum of the two transverse mode order numbers $n+m=r$ we refer to as $r$-resonances. The dashed line is an envelope for the resonance height.

The main resonance is the resonance for which all comb lines of the incident frequency comb simultaneously coincide with a cavity resonance. The side resonances drop in height and increase in width (width not indicated in Fig. 3) depending on the cavity finesse and spectral bandwidth, because different spectral parts of the frequency comb are on resonance for slightly different cavity lengths.

\section{Experimental realization}

\subsection{Experimental setup}

In order to demonstrate quasi-imaging, we employed the experimental setup shown in Fig. 4, and described in [1]. The Yb-based fiber laser system is described in [14]. The laser emits almost Fourier-limited $\tau=200$ fs pulses $(\tau \cdot \Delta v=0.39)$ with a central wavelength $\lambda=1040 \mathrm{~nm}$ and bandwidth $\Delta \lambda=7 \mathrm{~nm}$. The repetition rate is $v_{\text {rep }}=78 \mathrm{MHz}$. Throughout the experiments presented in this paper we used an incident average power of $P_{i n}=1.4 \mathrm{~W}$. Two spherical lenses were used to match the laser output mode to the enhancement cavity fundamental mode. The passive cavity of length $L=3.84$ m consists of eight mirrors. Mirrors M2-M8 are highly reflective $\left(R_{M}=0.99995\right)$ over the entire laser bandwidth and M1 is the input coupler $\left(R_{I C}=0.9986\right)$. Mirrors M5 and M6 are spherically curved (radius of curvature $R_{C}=150 \mathrm{~mm}$ ), all other mirrors are plane. This yields a Gaussian waist radius of $w_{0}=22 \mu \mathrm{m}$ in the middle of the stability range. All cavity mirrors exhibit low dispersion. In order to minimize round-trip dispersion, as well as to avoid nonlinearities in air, the cavity is placed in a vacuum chamber.

For resonant enhancement, the incident laser frequency comb modes have to spectrally overlap with the cavity resonances. As discussed in [3], due to the relatively narrow optical bandwidth of the femtosecond radiation, an active locking of the frequency comb to the cavity resonances can be realized by controlling only one of the two comb parameters, once they are brought in vicinity of optimum spectral overlap. The latter is performed by manually adjusting 
the enhancement cavity length and the carrier-envelope offset (CEO) phase by using a pair of wedges in the oscillator cavity. The locking electronics drives a piezoelectric transducer (PZT) varying the laser oscillator cavity length. The error signal for the locking is generated with the Hänsch-Couillaud scheme [15] from the signal reflected by the cavity input coupler. The only difference towards the setup described in [1] is a pinhole, enabling the spatial selection of the reflected beam profile for the generation of the error signal. This is particularly useful if the incident transverse mode does not match the excited cavity mode. The intracavity beam leaking through one of the highly reflecting cavity mirrors (M8) with a transmission of $1.65 \cdot 10^{-6}$ is characterized using a power meter and an optical spectrum analyzer. The circulating power $P_{\text {circ }}$ is calculated from the power $P_{\text {leak }}$ leaking through this mirror according to $P_{\text {circ }}$ $=P_{\text {leak }} / 1.65 \cdot 10^{-6}$.

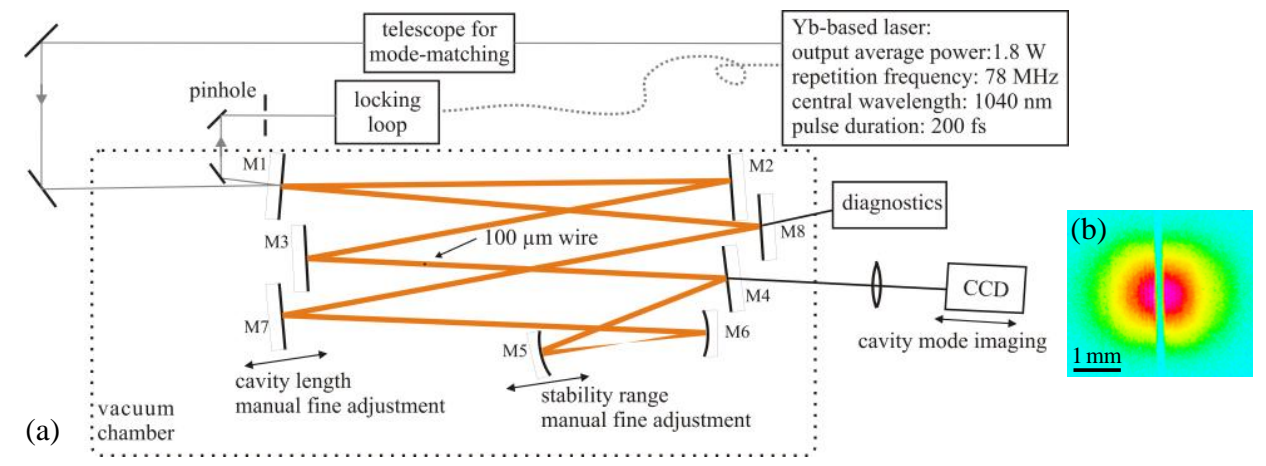

Fig. 4. Experimental setup. (a) Laser, enhancement cavity and locking scheme as in [1], diagnostics: power meter, optical spectrum analyzer. (b) Image of the $100 \mu \mathrm{m}$ thick on-axis wire with the cavity off resonance.

A copper wire was placed vertically on a motor-driven translation stage to function as an obstacle on the optical axis of the resonator. The position of the wire along the optical axis determines the positions of the on-axis intensity minima and maxima in the cavity. In order to yield the situation shown in Fig. 2 offering a geometrical access to the focus, the wire would have to be placed in front of the curved mirror behind the focus (M6). However, this position is not relevant for the demonstration of quasi-imaging. This is because the Gouy parameter $\psi$ $=3 \pi / 2$ acquired at one round trip starting at the wire is independent of its position. Therefore, the convenient implementation of the wire (400 $\mathrm{mm}$ before mirror M5) as well as the vertical orientation is chosen for good accessibility. The plane of the wire is imaged 1:1 with a lens ( $f$ $=400 \mathrm{~mm}$ ) on a CCD camera (see Fig. 4(b)). This allows for adjusting the wire to the optical axis and observing the beam profile at that position. To record the beam profile at different positions in the cavity the camera can be moved on a rail. The $100 \mu \mathrm{m}$ width of the wire equals $5 \%$ of the Gaussian beam diameter of $2 w=2.0 .99 \mathrm{~mm}$ at that position. An additional vertical aperture is placed in the cavity at the same longitudinal position and with a distance from the optical axis of roughly three times the Gaussian beam radius. This aperture serves as a spatial filter, i.e. it suppresses contributions of higher-order transverse modes other than $G H_{0,0}$ and $\mathrm{GH}_{4,0}$ to the circulating field.

A variation of the position in the stability range is achieved by displacing mirror M5 and compensating the change in the resonator length by displacing mirror M7 (see Fig. 4(a)). The cavity alignment is not significantly affected.

Throughout the experiments presented here, the q-parameter of the incident beam was adjusted for maximum spatial overlap with the fundamental mode of the enhancement cavity without the wire. The spatial overlap with higher-order transverse cavity modes is non-zero. We determined the spatial overlap with the fundamental mode by analysis of a scan pattern. We scanned the oscillator cavity length over more than one free spectral range and recorded the intracavity power measured with a photodiode placed behind mirror M8 and connected to an oscilloscope. For this experiment, we replaced mirrors M1 (the input coupler) and M8 by 
mirrors with a reflectivity of $R_{M}=0.99$. With the resulting finesse $F=100 \pi$ and with a scan time $t_{f s r}=7 \mathrm{~ms}$ for one free spectral range, the scan time over one resonance width $t_{\text {res }}=t_{f s r} / F$ $=22 \mu \mathrm{s}$ is about 17 times larger than the cavity build-up time $t_{b u}=(F / \pi) / v_{\text {rep }}=1.3 \mu \mathrm{s}$. This is long enough to allow for a full build-up of the intracavity radiation for each transverse mode, and short enough to avoid distortions by mechanical vibrations. We assume a linear behavior of the scanning PZT. Then the intracavity power integrated over the illumination time of a transverse mode or a group of transverse modes is proportional to the spatial overlap of the incident beam with the respective mode or group of modes. Transverse modes with mode order higher than 4 were not measurable and therefore not considered. In this manner, the spatial overlap of the incident beam with the fundamental mode was estimated to be $U=0.94$.

\subsection{Enhancement of the cavity modes $\mathrm{GH}_{0,0}$ and $\mathrm{GH}_{1,0}$}

In two first experiments, we locked the cavity without the wire to the fundamental mode $\left(G H_{0,0}\right)$ and, subsequently, to the $G H_{1,0}$ mode. The purpose of these experiments was to characterize the empty cavity, in particular the round-trip loss and impedance matching. Also, the elaborate evaluation of finesse and overlap from enhancement and coupling was tested for consistency.

With the fundamental mode we achieved stable locking over several minutes with a circulating average power of $P_{\text {circ }}=1950 \mathrm{~W}$. This corresponds to a power enhancement of $P_{\text {circ }} / P_{\text {in }}=1400$. The circulating power fluctuates in time and repeatedly drops to $\sim 0.8$ of the maximal value. We attribute this strong fluctuation to the relatively slow PZT used for locking (measured mechanical bandwidth $<20 \mathrm{kHz}$ ) in conjunction with the high cavity finesse. This was confirmed by decreasing the cavity finesse, which led to a significant decrease of the fluctuations. Furthermore, this is not a fundamental limitation of cavity enhancement, since a larger locking bandwidth can be achieved by advanced design of PZT-actuated mirrors [16]. The time-averaged circulating power $P_{\text {circ }}$ was measured with a power meter. We assume that the maximal power level $P_{\text {circ }}{ }^{\text {max }}$ is determined by the loss and overlap, and the dropping is due to a deviation from resonance. This maximum value is $P_{\text {circ }}{ }_{\max }=1.1 \cdot P_{\text {circ }}$ (compare Fig. 5(a)). The corresponding maximum level of the enhancement, which was used to calculate the loss and the overlap, is $E=P_{\text {circ }}{ }^{\max } / P_{\text {in }}=1540$. The coupling ratio $K$ of the power coupled into the cavity to the incident power was determined from the drop of the signal reflected from the input coupler compared to the unlocked case. This signal was measured with a photodiode. The maximum level of the coupling was determined to be $K=0.70$. From these values the round trip loss factor is calculated to be $R=1-K / E=0.99955$. It follows for the cavity finesse $F=2 \pi /\left(1-R_{I C} R\right)=3400$. The difference between the measured enhancement $E=1540$ and the enhancement expected from the loss $E_{R}=\left(1-R_{I C}\right) /\left(1-\left(R_{I C} R\right)^{1 / 2}\right)^{2}=1630$ can be attributed to an incomplete spatial overlap of $U=E / E_{R}=0.94$, since a complete spectral overlap was confirmed by measuring the incident and circulating spectra. This spatial overlap matches the value estimated from the scan. The pulse duration was measured to be $\tau=200 \mathrm{fs}$.

We then locked the cavity without the wire to the $G H_{1,0}$ mode. Because the $G H$ modes form an orthogonal set of modes, the spatial overlap of an incident $\mathrm{GH}_{0,0}$ mode with the circulating $G H_{1,0}$ mode vanishes, unless the incident beam has a tilt or transverse offset with respect to the cavity optical axis. In the experiment, the q-parameter was left the same as before and the beam was tilted horizontally to optimize the spatial overlap. The maximum spatial overlap for an incident Gaussian beam with eigen-q-parameter is easily calculated to be $U=$ $1 / e=0.37$ for a tilt angle of $\gamma=2 /(k \cdot w)$ or an offset of $\Delta x=w$ with the wave number $k$ and Gaussian beam radius $w$. The pinhole in the locking scheme was used to select one of the two lobes in the reflected signal. The CEO phase of the incident pulse train had to be adjusted for maximum spectral overlap of the frequency comb modes with the cavity resonances corresponding to the $G H_{1,0}$ mode. This is due to the fact that the cavity resonances for mode order $n$ $=1$ are shifted by the Gouy parameter $\psi_{x}$ compared to the fundamental mode (see Fig. 3). A power enhancement of $P_{\text {circ }} / P_{\text {in }}=550, P_{\text {circ }}^{\max }=1.1 \cdot P_{\text {circ }}$ and a coupling of $K=0.28$ were measured. The finesse is determined to be $F=3400$, which is the same as for the fundamental mode. The spatial overlap is $U=0.37$, which matches the value calculated for an incident 
Gaussian beam. The smaller power enhancement compared to the fundamental mode is due to the reduced spatial overlap alone, the resonator loss and therefore the finesse is identical in both cases. As in the case of the fundamental mode, the entire spectrum was coupled to the $G H_{1,0}$ mode and evenly enhanced.
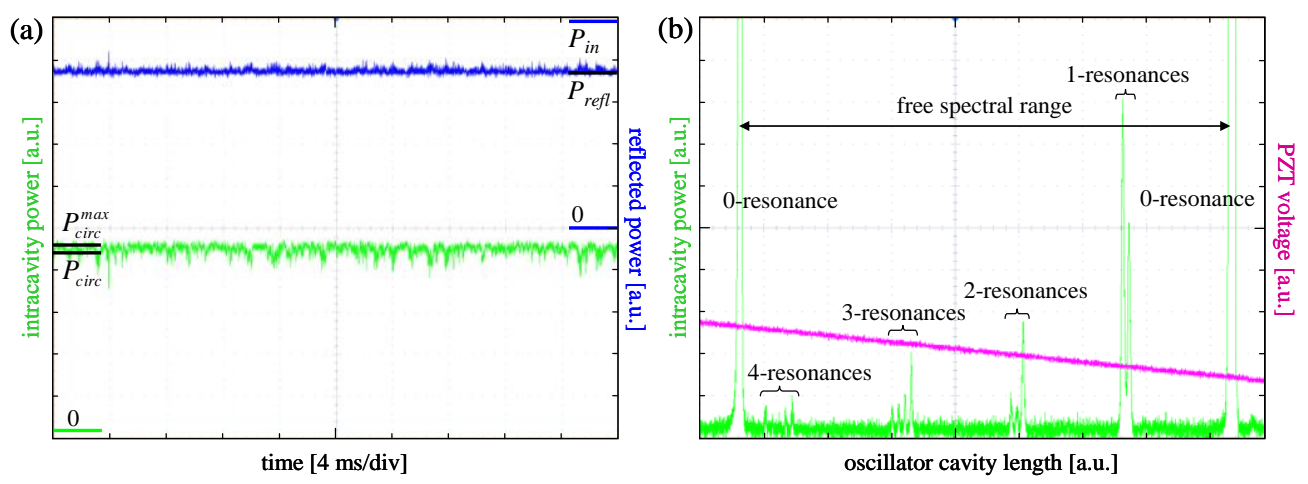

Fig. 5. (a) Lock of the slit mode with the wire on the optical axis. Transmitted signal (green, 50 $\mathrm{mV} /$ div) and reflected signal (blue, $500 \mathrm{mV} / \mathrm{div}$ ). The graph shows the fluctuation of the circulating power with time and the meaning of $P_{\text {circ }}$ and $P_{\text {circ }}{ }^{\max }$. (b) Measured resonances (transmitted signal, green, $1 \mathrm{mV} / \mathrm{div}$ ) for a linear scan (PZT signal, magenta, $5 \mathrm{~V} / \mathrm{div}$ ) of the oscillator cavity length without wire (compare Fig. 3). The abscissa is in fact a time axis with $1 \mathrm{~ms} / \mathrm{div}$. Transverse mode resonances up to order 4 are visible. Modes with the same sum of the two transverse mode order numbers $m+n=$ const. can be clearly distinguished. The Gouy parameter is $\psi_{x}=0.78 \cdot 2 \pi$ and $\psi_{y}=0.76 \cdot 2 \pi$ assuming a linear response of the PZT.

\subsection{Quasi-imaging: enhancement of slit modes with an on-axis obstacle}

In the final experiment the incident beam was aligned to the cavity optical axis and the CEO phase was adjusted for maximum spectral overlap with the cavity resonances of the fundamental mode. The wire was placed as an obstacle on the optical axis, which can be done with high precision by monitoring the image of the wire while the cavity is not locked (see Fig. 4(b)). In order to achieve the transverse mode degeneracy, we adjusted the position in the stability range by changing the distance $d$ between the curved mirrors by displacing mirror M5 and compensating the change in the resonator length by displacing mirror M7. The position in the stability range can be read from the scan pattern of the cavity with the wire removed from the beam path, as is discussed in Section 2.3. Figure 5(b) shows a recorded scan pattern.
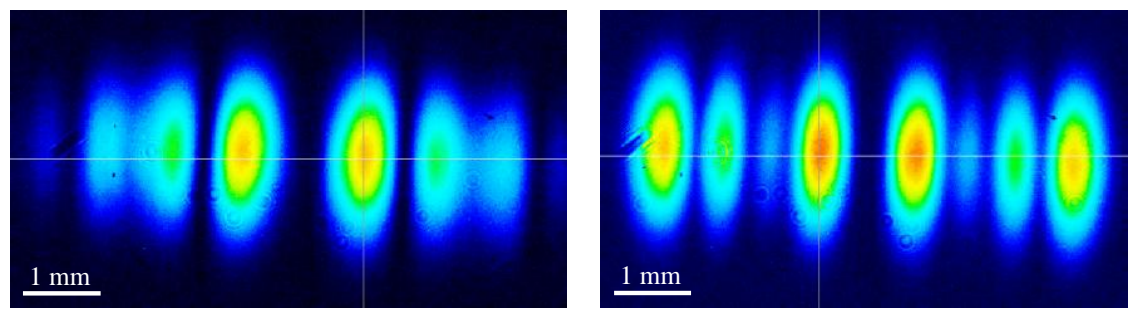

Fig. 6. Different intensity distributions at the position of the wire on the optical axis in the locked cavity recorded at different positions in the stability range. Besides the wire, no additional obstacle is used here.

In the locked case, different field distributions with different numbers of lobes were observed, depending on the position in the stability range (see Fig. 6). Field distributions with more than the four lobes for the simple slit mode depicted in Fig. 1(a) involve the contribution of higher-order transverse modes $\left(\mathrm{GH}_{8,0}\right.$ and $\left.\mathrm{GH}_{12,0}\right)$. In order to suppress these contributions, a vertical aperture was placed in the cavity and moved towards the optical axis until additional lobes besides the four were suppressed. This was the case for a distance from the optical axis of about three times the Gaussian beam radius (see Fig. 7(a)). 

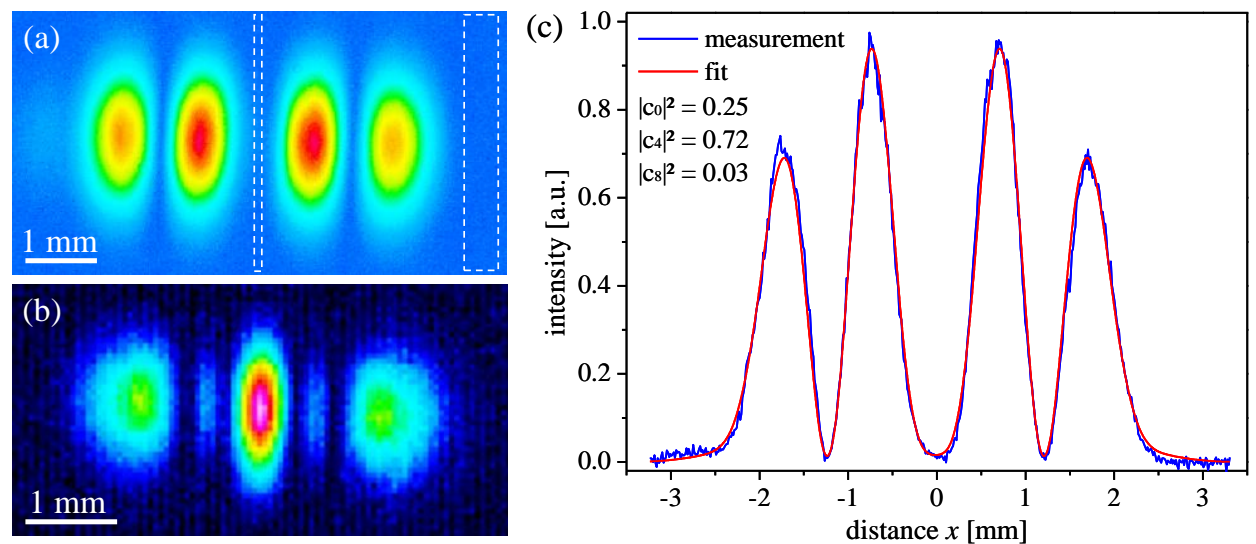

Fig. 7. Measured intensity distribution of the excited slit mode. (a) Image of the plane with the wire on the optical axis and spatial filtering aperture, Gaussian beam radius $w=0.99 \mathrm{~mm}$. Wire and aperture are indicated by dashed lines. (b) Image of a position with maximal intensity on the optical axis, which is $2.4 \mathrm{~m}$ before the wire, Gaussian beam radius $w=0.77 \mathrm{~mm}$. (c) Fit of the mode combination $c_{0} \cdot G H_{0,0}+c_{4} \cdot G H_{4,0}+c_{8} \cdot G H_{8,0}$ with complex coefficients to a cut through the intensity distribution from (a). The contribution of the three $G H$ modes is indicated.

As in the previous experiment, we used the pinhole in the locking scheme to select one of the lobes for the generation of the error signal. We went through a region in the stability range around the degeneracy by varying the distance $d$ by some tens of $\mu \mathrm{m}$. Figure $8(\mathrm{a})$ shows the enhancement as a function of the detuning from the quasi-imaging condition in terms of the Gouy parameter $\psi_{x}$ and of the distance $d$ between the curved mirrors. We have derived a theoretical model for the detuning curve. However, the model exceeds the scope of this paper and will be described in [13]. The experimentally measured width (FWHM) of the detuning curve is $\Delta \delta=17 \mu \mathrm{m}$. On the one hand, this is a small fraction of the entire stability range of $3.2 \mathrm{~mm}$. On the other hand, it is so broad that the quasi-imaging can be easily adjusted manually and does not demand for an active control of the distance $d$.
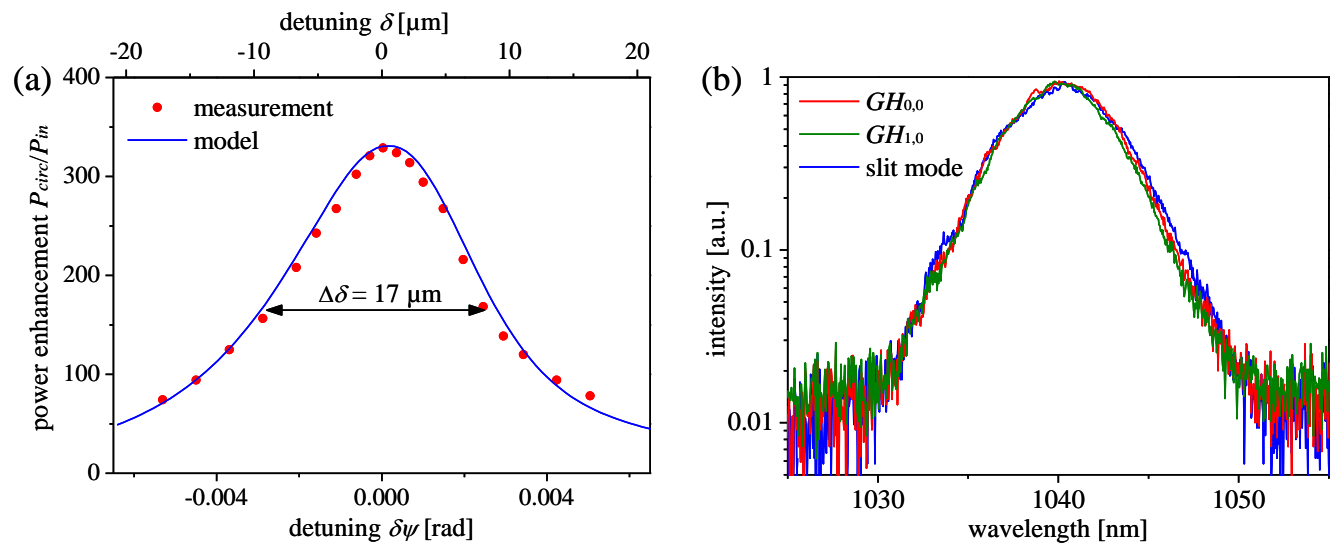

Fig. 8. (a) Power enhancement $P_{\text {circ }} P_{\text {in }}$ versus detuning from the resonator degeneracy. The model used here will be described in [13]. (b) Normalized intracavity spectra of the $G H_{0,0}$, $\mathrm{GH}_{1,0}$ and slit mode (logarithmic scale). The curves show that the spectral coupling to the excited transverse modes is identical in all cases.

For maximum power enhancement $P_{\text {circ }} / P_{\text {in }}=330$ at circulating power $P_{\text {circ }}=460 \mathrm{~W}$, a maximum level of the circulating power $P_{\text {circ }}{ }^{\max }=1.08 \cdot P_{\text {circ }}$ (see Fig. 5(a)) and a coupling of $K$ $=0.24$ were measured. The finesse is determined to be $F=3000$, which represents only a small decrease compared to the cavity without the wire $F=3400$. The additional round-trip loss at the wire amounts to $2.3 \cdot 10^{-4}$. With the wire the enhancement expected from the loss is 
$E_{R}=1290$. This represents a reduction by 0.79 compared to the cavity without the wire. However, the smaller power enhancement compared to the fundamental mode is mainly due to the reduced spatial overlap. Figure 7 shows the measured intensity distribution of the enhanced field at the position of the wire and at a position of maximum intensity on the optical axis. A fit of a mode combination of $G H_{0,0}, G_{4,0}$ and $G H_{8,0}$ with complex coefficients to the intensity distribution yields a power fraction of $\left|c_{0}\right|^{2}=0.25$ in the fundamental mode. The power fraction in the $G H_{8,0}$ mode is only $\left|c_{8}\right|^{2}=0.03$, because it is suppressed by the aperture. The power fraction of $\left|c_{0}\right|^{2}=0.25$ defines the expected spatial overlap with an incident Gaussian beam with the eigen-q-parameter. The spatial overlap calculated from enhancement and coupling is $U=0.27$, which is in good agreement with the value of $\left|c_{0}\right|^{2}$. The entire spectrum was coupled to the slit mode in the cavity and evenly enhanced (see Fig. 8(b)).

For a thicker wire with $220 \mu \mathrm{m}$ width (which equals $11 \%$ of the Gaussian beam diameter) placed at the same position we measured a power enhancement $P_{\text {circ }} / P_{\text {in }}>300$. Table 1 summarizes the results for the different resonator modes.

Table 1. Finesse, Enhancement, Spatial Overlap and Pulse Duration for the Different Resonator Modes

\begin{tabular}{ccccc}
\hline & $G H_{0,0}$ & $G H_{1,0}$ & & slit mode \\
\cline { 2 - 3 } finesse $F$ & 3400 & 3400 & & with wire \\
\hline $\begin{array}{c}\text { enhancement } \\
P_{\text {circ }} / P_{\text {in }}\end{array}$ & 1400 & 550 & & 3000 \\
\hline spatial overlap $U$ & 0.94 & 0.37 & & 0.27
\end{tabular}

The power enhancement in the experiment is limited by the imperfect spatial overlap of $U$ $=0.27$ for the simple slit mode. The spatial overlap is expected to reach 0.44 for an incident Gaussian beam with an adapted q-parameter [13]. A cylindrical telescope has to be used then for mode matching. Theoretically, the spatial overlap can reach unity if the incident field is adequately shaped. Beam shaping can e.g. be achieved with free form optics. The increase of the spatial overlap is a problem which can be solved outside the enhancement cavity.

\section{Further discussion}

The experiment presented here shows that it is possible to combine simultaneously resonant transverse modes in a stable degenerate cavity to form a field distribution reproducing an onaxis hole or slit at every round trip. A hole or slit is also reproduced in an imaging resonator. However, unlike imaging, quasi-imaging can be achieved in a stable resonator. In the simple case of a ring resonator with two focusing mirrors and a constant length, only one parameter, namely the distance of the focusing mirrors, has to be adjusted to achieve quasi-imaging. An imaging resonator is understood to be telecentrically imaging with magnification \pm 1 , because only then a field distribution can be reproduced after one resonator round trip. This can be achieved in a symmetrical confocal resonator, for which the length has to equal the radius of curvature [9]. If one wants to choose the resonator length and the radius of curvature independently, further curved mirrors have to be added, leading to additional distances which have to be adjusted [17]. An imaging resonator is not stable in the sense that it does not possess a distinguished eigen-q-parameter. Instead, any q-parameter is reproduced after a resonator round trip. Both concepts, imaging and quasi-imaging, are promising as geometrical output coupling methods for intracavity-generated XUV radiation, offering the prospect of average and peak power scalability in enhancement cavities for HHG.

In order to achieve a geometrical on-axis access to the cavity, the obstacle can be an opening in a cavity mirror. This is preferably a curved mirror, if an access to the focus is desired. The fabrication of a standard quarter-inch-thick mirror with a hole or slit with dimensions on the order of $100 \mu \mathrm{m}$ is challenging, nevertheless first experiments are promising. Fabrication of such mirrors is currently being pursued by direct laser drilling with a frequency doubled nslaser and a sub-ns-laser [18] and by in-volume selective laser etching (ISLE). 
The on-axis access to the cavity could be used for output coupling of high harmonics generated in a gas jet near the focus. The suitability of the field distribution for efficient high harmonic generation still has to be shown. As can be seen from Fig. 2, the field distribution changes its shape rapidly in the region around the focus. Therefore, the coherence length is somewhat reduced compared to the fundamental mode with the same Rayleigh length. The field one Rayleigh length from the focus has a central lobe on the optical axis containing 0.45 of the power. This central lobe has an elliptical shape, as can be seen in Fig. 7(b). In the focal plane the two central lobes contain 0.59 of the power. These values hold for the simple slit mode of $\mathrm{GH}_{0,0}$ and $\mathrm{GH}_{4,0}$ alone. Contributions of higher-order transverse modes can change the shape of the field. This can be manipulated by apertures, by the shape of the incident field and by the position in the stability range. The interaction region for HHG could be limited to one of these two regions. The lobes in the focal plane are in phase, which means that the harmonic signal will exhibit a maximum on the optical axis in the far field. In contrast, the $G H_{1,0}$ mode, as well as other $G H$ or $G L$ modes with vanishing intensity on the optical axis, are unfavourable for HHG, because the lobes oscillate with opposite phase and the harmonic signal vanishes on the optical axis [8].

The geometrical on-axis access to a high-finesse cavity could also be used for other applications besides output coupling of high harmonics, e.g. for Thomson back-scattering at electrons for the generation of coherent X-radiation [19]. Here, a cavity can be used to enhance the fs-radiation which is back-scattered at a high-energy electron beam. A geometrical access could be used for output coupling of the X-radiation and/or the input of the electron beam.

The rapidly changing shape of the intensity distribution could be advantageous for other applications. E.g. by the combination of two $G L$ modes, an intensity minimum in the focus can be formed, which is strongly localized in radial as well as in axial direction. Such a field distribution, possibly enhanced in a cavity, can serve as a dipole trap for neutral atoms [20]. Moreover, such field distributions can also be excited in an active resonator by the use of a degeneracy of transverse modes and adapted apertures.

\section{Conclusion}

We demonstrated quasi-imaging in a high-finesse femtosecond enhancement cavity. This is a stable cavity with a degeneracy of transverse modes and an obstacle in the beam path. The obstacle exacts a hole in the field distribution. This hole is reproduced after a resonator round trip due to the mode degeneracy. Therefore, only small loss is introduced by the obstacle. The concept allows for a geometrical on-axis access to the cavity, which could be used as a dispersion-free and peak as well as average power scalable method for output coupling of intracavity-generated high harmonics with increasing efficiency for decreasing wavelengths. In order to achieve quasi-imaging, the distance of the curved mirrors in the bow-tie ring cavity has to be adjusted with a precision of a few $\mu \mathrm{m}$, which is only a small fraction of the stability range but still can be attained manually.

We excited a field distribution avoiding a wire on the optical axis and at a different position exhibiting an on-axis intensity maximum. Compared to the fundamental mode with $F=$ 3400 , the cavity finesse was only slightly reduced to $F=3000$ by the insertion of the wire with a width equal to $5 \%$ of the Gaussian beam diameter. This corresponds to an additional round-trip loss of $2.3 \cdot 10^{-4}$. The decrease of the power enhancement from $P_{\text {circ }} / P_{\text {in }}=1400$ to $P_{\text {circ }} / P_{\text {in }}=330$ is mainly due to the imperfect spatial overlap, which could be increased by shaping the incident beam.

\section{Acknowledgments}

This work was supported within the cooperation project KORONA between the Max-PlanckInstitut für Quantenoptik (MPQ) and Fraunhofer-Institut für Lasertechnik (ILT). We thank the fiber laser group at the Institute of Applied Physics at Friedrich-Schiller-University Jena for providing the laser source for the experiments. 


\section{Chapter 7}

\section{Appendix}

\subsection{Experiments Involving Enhancement Cavities}

Continuous-wave passive cavities with power enhancement factors on the order of $10^{5}$ have been demonstrated in the early nineties for sensitivity enhancement of optical losses measurements, see e.g. [142]. With the advent of Ti:Sa-based frequency combs, ultrashort pulses were enhanced for the first time for MHz-repetition-rate HHG [17, 18]. Due to their broad optical bandwidth, Ti:Sa lasers enable high peak intensities through the short pulse duration rather than the average power. The shortest intra-cavity pulse duration of $27 \mathrm{fs}$ has been achieved with a power enhancement of 50 by seeding a broadband cavity with $20 \mathrm{fs}$ pulses [17]. More recently, Yb-based frequency combs were employed for intra-cavity HHG due to their significantly larger average power [19,21]. So far, the largest circulating powers in an ultrafast enhancement cavity lie in the multi-10-kW range 33] and have been demonstrated with the Yb-based system presented in this thesis. For some applications, the only drawback of Yb-based seeding lasers towards Ti:Sa systems is the relatively narrow available optical bandwidth. However, nonlinear pulse compression of the seeding pulses offers the prospect of the enhancement of few-cycle Yb-based pulses (see also Section 5.1.2).

Further passively enhanced laser sources include frequency-doubled Ti:Sa and Yb-based systems in the UV [137] and in the green spectral regions [22], respectively. However, the available mirrors for these wavelengths reach significantly lower reflectivity values than in the infrared, limiting the achievable finesse. The UV enhancement cavity, which also includes a nonlinear crystal, supports $7 \mathrm{~W}$ of circulating average power at a repetition rate of $81 \mathrm{MHz}$ enabling a powerful source for experiments with entangled multiphoton states. The green enhancement cavity is an ongoing project led by Birgitta Bernhardt in the group of Prof. Hänsch at the MPQ, in collaboration with our group. The goal of this experiment is to increase the available power of frequency combs at around $60 \mathrm{~nm}$ wavelength for highprecision XUV spectroscopy. Using green light for HHG promises a significant increase in the conversion efficiency due to the shorter fundamental wavelength, see Section 2.2.2. So far, the circulating power in the cavity is limited to a few hundred watts due to a mirror degradation phenomenon in vacuum, which is still being investigated 22, 109. 


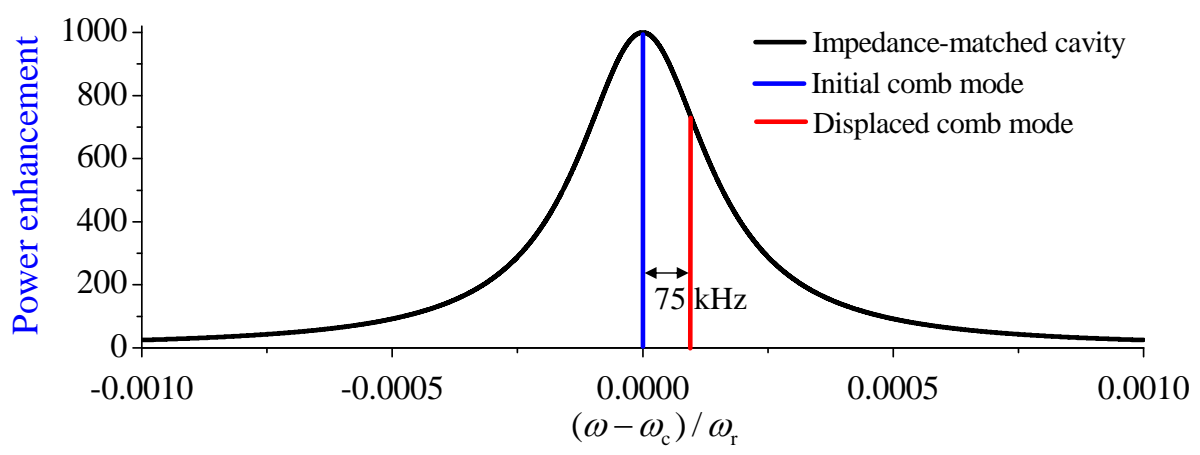

Figure 7.1: Enhancement of mode number 3695666 (blue line) of a $78-\mathrm{MHz}$ repetitionrate frequency comb with the central wavelength of $1040 \mathrm{~nm}$ in an impedance-matched enhancement cavity with a power enhancement factor of 1000. A frequency shift $\Delta f=$ $7.5 \mathrm{kHz}$ of the enhanced mode (red line) leads to an enhancement reduction to $\sim 725$. This comb shift corresponds to either a repetition frequency change of $2 \mathrm{mHz}$ (equivalent to a roundtrip length change of $0.1 \mathrm{~nm}$ ) or to a CE phase slippage of $0.6 \mathrm{mrad}$.

\subsection{An Example of Comb-Cavity Detuning}

To exemplify the effect of a detuning of the cavity length on the enhancement, we consider the case of a $78-\mathrm{MHz}$ repetition-rate frequency comb, with the central optical wavelength of $1040 \mathrm{~nm}$. These values are chosen in accordance with the system presented in this thesis. Furthermore, we consider an impedance-matched enhancement cavity with a power enhancement factor of 1000, so that the central comb mode (with wavelength $1040 \mathrm{~nm}$ and mode number 3695666) is locked to a corresponding cavity resonance, see blue line in Fig. 7.1. An optical frequency shift of the enhanced comb line of $\Delta f=7.5 \mathrm{kHz}$ means a reduction of the enhancement to $\sim 725$, see red line in Fig. 7.1. This shift corresponds to either a repetition frequency change of $\Delta f / 3695666=2 \mathrm{mHz}$ of the seeding comb, which is equivalent to a roundtrip length change of $0.1 \mathrm{~nm}$ or to a CE phase slippage of $\Delta \varphi=2 \pi \cdot 7.5 \cdot 10^{3} /\left(78 \cdot 10^{6}\right) \mathrm{rad}=0.6 \mathrm{mrad}$. 


\subsection{Overview of XUV Output Coupling Methods}

In the following we give a brief overview of the existing methods for coupling out intracavity generated XUV radiation, i.e. spatially splitting the generated high harmonics (HH) from the circulating fundamental radiation (FR).

\section{Brewster Plates}

- Mode of operation: The HH experience total internal reflection at a thin plate placed under Brewster's angle for the FR.

- Publications: 17, 18, 20; discussed in this thesis in Sections 4.4.1 and 5.2.1.

- Experimentally demonstrated: yes.

- Output coupling efficiency at $60 \mathrm{~nm}$ : roughly $10 \%$, at $10 \mathrm{~nm}$ : $\ll 0.1 \%$.

- Average power scalability: poor due to poor heat conduction. Currently limited at roughly $3 \mathrm{~kW}$. Peak power scalability: poor due to nonlinearities which increase with thickness, (but can in principle be compensated for).

\section{GIP - Grazing-Incidence Plates}

- Mode of operation: Similar to the Brewster plate, but under grazing incidence and (optionally) for s polarization. High transmission for the FR through the GIP is obtained by anti-reflection coatings on both sides of the plate.

- Publications: 108; not further discussed in this thesis.

- Experimental demonstration: has not been included in a cavity yet.

- Output coupling efficiency at $60 \mathrm{~nm}$ : roughly $60 \%$, at $10 \mathrm{~nm}$ : roughly $5 \%$.

- Average and peak power scalability: not yet investigated. Expected to be similar to the Brewster plate, or poorer due to the additional coatings.

- Additional nonlinearities and dispersion: similar to the Brewster plate.

\section{WOMOC - Wedge-on-Mirror Output Coupler}

- Mode of operation: A thin plate is attached to the surface of a rigid, highly reflecting mirror, providing improved thermal conduction. The FR impinges on the WOMOC under Brewster's angle, penetrates the plate, is reflected by the mirror and exits the plate. The plate is wedged under a small angle, ensuring the spatial separation of the $\mathrm{HH}$ from the FR.

- Publications: 35]; discussed in this thesis in Sections 4.4 .2 and 5.2.1. 
- Experimental demonstration: has not been included in a cavity yet.

- Output coupling efficiency at $60 \mathrm{~nm}$ and at $10 \mathrm{~nm}$ : like Brewster plate.

- Average and peak power scalability: not yet investigated. Expected to be better than Brewster plate because (i) the penetrated material is attached over a large area to a robust optic and (ii) the penetrated material thickness is decreased.

- Additional nonlinearities and dispersion: expected to be low or negligible.

\section{Nanograting}

- Mode of operation: A nanostructure written in the last layer of the dielectric multilayer coating of the cavity mirror following the HHG focus diffracts the $\mathrm{HH}$ away from the resonator optical axis. For the FR the element acts as a high reflector.

- Publications: 19,36]; discussed in this thesis in Section 4.4 .4

- Experimental demonstration: yes.

- Output coupling efficiency at $60 \mathrm{~nm}$ and at $10 \mathrm{~nm}$ : similar to Brewster plate or better. Can be optimized for a specific wavelength region.

- Average power scalability: expected to be very good. Peak power scalability: this is a limitation due to the nonlinearities enhanced by the nanostructure.

- Additional nonlinearities: not known. Additional dispersion: none to the IR. However, the output coupled harmonics are spatially dispersed.

\section{Hole in Mirror after HHG Focus and Use of Fundamental Mode}

- Mode of operation: The HH, whose divergence decreases with increasing order, are directly coupled out through a small on-axis hole in the cavity mirror following the HHG focus. If the hole is small enough, the losses introduced to the circulating FR still allow for a high enhancement.

- Publications: 106, 107]; discussed in this thesis in Section 5.2.2.

- Experimental demonstration: while a cavity with such a mirror has been demonstrated, output coupling of high harmonics through the hole has not yet been reported.

- Output coupling efficiency at $60 \mathrm{~nm}$ and at $10 \mathrm{~nm}$ : depends on the directional characteristic of the XUV radiation. It is expected to increase for lower wavelengths.

- Average power scalability: not yet investigated. It is expected to depend critically on the quality of the hole edge. Peak power scalability: not yet investigated. It is expected to be good. 
- Additional nonlinearities and dispersion: none.

\section{Hole or Slit in Mirror after HHG Focus and Quasi-Imaging}

- Mode of operation: Using quasi-imaging, for the FR a field distribution is excited in the cavity, which has an on-axis maximum near the focus for HHG and simultaneously avoids a hole or slit in the cavity mirror following the focus. The $\mathrm{HH}$ generated in the central lobe of the on-axis maximum are expected to propagate along the axis and exit the cavity through the opening in the mirror.

- Publications: 37, 143]; discussed in this thesis in Sections 2.1.4, 4.4.3 and 5.2.2.

- Experimental demonstration: a proof of principle of quasi-imaging was demonstrated. However, the suitability of quasi-imaging modes for HHG as well as output coupling of XUV light through an opening in a mirror following the focus has not yet been shown.

- Output coupling efficiency at $60 \mathrm{~nm}$ and at $10 \mathrm{~nm}$ : depends on the directional characteristic of the generated XUV radiation, which still needs to be investigated. It is expected to be very high for lower wavelengths.

- Average power scalability: not investigated properly yet. Preliminary results in our lab show $5 \mathrm{~kW}$. It is expected to depend on the quality of the slit edge. Peak power scalability: not investigated yet. It is expected to be good.

- Additional nonlinearities and dispersion: none.

\section{Non-Collinear HHG}

- Mode of operation: Two FR pulses collide non-collinearly in a high-intensity region of one (or two) cavity/cavities, creating an interference pattern. For properly chosen parameters, the emitted $\mathrm{HH}$ propagate along the bisector of the angle formed by the two propagation paths of the FR.

- Publications: 106, 116, 117]; not further discussed in this thesis.

- Experimental demonstration: a proof of principle for the single-pass non-collinear HHG has been shown. However, this technique has not yet been shown in conjunction with enhancement cavities.

- Output coupling efficiency at $60 \mathrm{~nm}$ and at $10 \mathrm{~nm}$ : not investigated yet but expected to be very good.

- Average and peak power scalability: not investigated yet but expected to perform very well.

- Additional nonlinearities and dispersion: none. 


\subsection{Data Archiving}

The raw data used in this thesis, the codes employed for simulations and interpretation, as well as the $\mathrm{H}_{\mathrm{E}} \mathrm{T}_{\mathrm{E}} \mathrm{X}$ source code of the thesis can be found on the Data Archive Server (DAS) at the Max Planck Institute for Quantum Optics, Laboratory for Attosecond Physics (LAP), in the following directories:

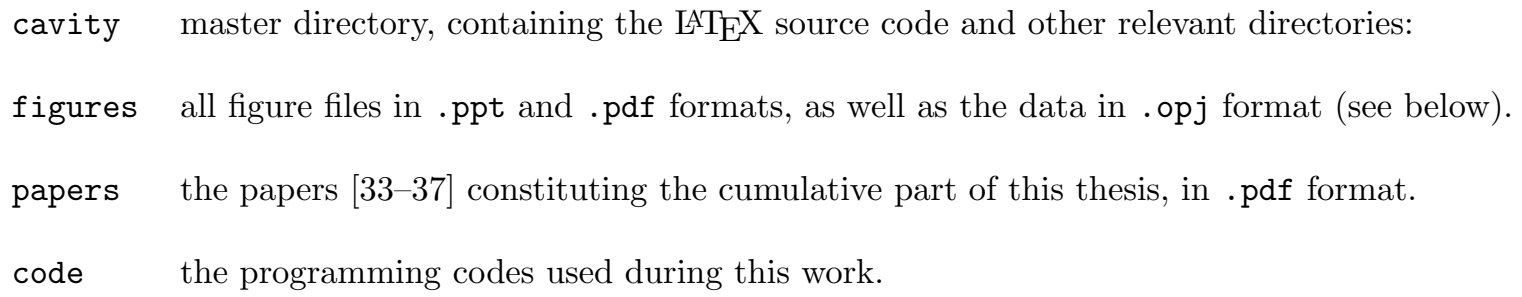

In the following, a complete list of the figures in the directory figures containing measurement and/or simulation data is given, together with the codes used. The papers 33 37 are archived separately.

Fig. 2.5 calculated data routine

Fig. 2.6 calculated data routine

Fig. 2.8 calculated data routine

Fig. 2.9 calculated data routine

Fig. 4.3 oscilloscope screenshot

Fig. 4.6 oscilloscope screenshot

Fig. 4.7 oscilloscope screenshot

Fig. 4.8 oscilloscope screenshot

Fig. 4.9 measurement data (raw)

Fig. 4.12 measurement data (raw)

Fig. 4.13 measurement data (screenshots)

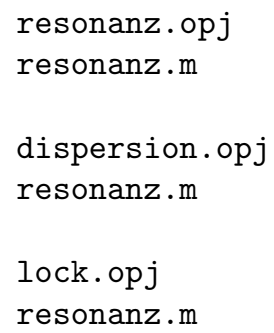

lock.opj

resonanz.m

Origin data embedded in calc.ppt

comparison.m (uses fwhm.m)

frequenzmessung.ppt

scanlock_i.ppt

scan $1 \mathrm{~mm}$.ppt

scanrefl.ppt

embedded in: THG.ppt

embedded in: enh_1mm.ppt

$1 \mathrm{~mm} \cdot \mathrm{ppt}$ 
Fig. 4.16 data

Fig. 4.17 data

Fig. 2.6 simulation data routine

Fig. 5.2 simulation data routine

Fig. 5.3 simulation data routine

$G D D$ parameter files

Fig. 7.1 calculated data routine embedded in: xuv_prop.ppt

embedded in: xuv_spectra.ppt

lewenstein_EF.opj

implemented by Ernst Fill, described in text

radius.opj

calculated by Jan Kaster, standard ABCD

matrix formalism

Simulation data embedded in: short_pulses.ppt calculated by Simon Holzberger, standard passive cavity model

R0310030_GDD_R_Or̃.dat

fluct.opj

resonanz.m.

Additional programming code, related to the subject of this thesis is archived in the directory code and described in the following:

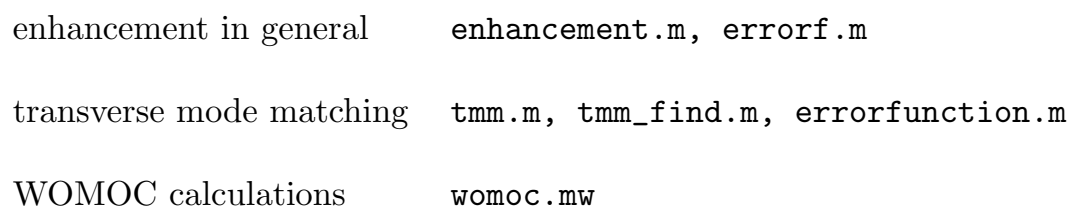




\section{Bibliography}

[1] T. Walther and H. Walther, Was ist Licht? (C. H. Beck, München, 1999).

[2] A. Siegman, Lasers (University Science Books, Sausalito, California, 1986).

[3] F. Träger, Springer Handbook of Lasers and Optics (Springer, New York, 2007).

[4] D. Attwood, Soft X-rays and Extreme Ultraviolet Radiation (Cambridge University Press, Cambridge, 1999).

[5] P. Jaegle, Coherent Sources of XUV Radiation (Springer, New York, 2006).

[6] M. J. Weber, Handbook of laser wavelengths (CRC Press, 1999).

[7] A. Ashkin, G. D. Boyd, and J. M. Dziedzic, "Resonant optical second harmonic generation and mixing," IEEE J. Quantum Electron. 2, 109 (1966).

[8] K. Fiedler, S. Schiller, R. Paschotta, P. Kürz, and J. Mlynek, "Highly efficient frequency-doubling with a doubly resonant monolithic total-internal-reflection ring resonator," Opt. Lett. 18, 1786 (1993).

[9] Z. Y. Ou and H. J. Kimble, "Enhanced conversion efficiency for harmonic-generation with double-resonance," Opt. Lett. 18, 1053 (1993).

[10] R. Paschotta, P. Kürz, R. Henking, S. Schiller, and J. Mlynek, "82\% efficient continuous-wave frequency doubling of $1.06 \mu \mathrm{m}$ with a monolithic $\mathrm{MgO}: \mathrm{LiNbO} 3$ resonator," Opt. Lett. 19, 1325 (1994).

[11] V. P. Yanovsky and F. W. Wise, "Frequency doubling of 100-fs pulses with 50\% efficiency by use of a resonant enhancement cavity," Appl. Phys. Lett. 19, 1952 (1994).

[12] E. Peters, S. A. Diddams, P. Fendel, S. Reinhardt, T. W. Hänsch, and T. Udem, "A deep-UV optical frequency comb at 205 nm," Opt. Express 17, 9183 (2009).

[13] J. Mes, E. J. van Duijn, R. Zinkstock, S. Witte, and W. Hogervorst, "Third-harmonic generation of a continuous-wave Ti:Sapphire laser in asternal resonant cavities," Appl. Phys. Lett. 82, 4423 (2003). 
[14] B. Couillaud, T. W. Hänsch, and S. G. MacLean, "High power CW sum-frequency generation near $243 \mathrm{~nm}$ using two intersecting enhancement cavities," Opt. Commun. 50, 127 (1984).

[15] M. Theuer, D. Molter, K. Maki, C. Otani, J. A. L'huillier, and R. Beigang, "Terahertz generation in an actively controlled femtosecond enhancement cavity," Appl. Phys. Lett. 93, 041119 (2008).

[16] F. Ilday and F. X. Kärtner, "Cavity-enhanced optical parametric chirped-pulse amplification," Opt. Lett. 31, 637 (2006).

[17] C. Gohle, T. Udem, M. Herrmann, J. Rauschenberger, R. Holzwarth, H. A. Schuessler, F. Krausz, and T. W. Hänsch, "A frequency comb in the extreme ultraviolet," Nature 436, 234 (2005).

[18] R. Jones, K. D. Moll, M. J. Thorpe, and J. Ye, "Phase-Coherent Frequency Combs in the Vacuum Ultraviolet via High-Harmonic Generation inside a Femtosecond Enhancement Cavity," Phys. Rev. Lett. 94, 193201 (2005).

[19] D. C. Yost, T. R. Schibli, and J. Ye, "Efficient output coupling of intracavity high harmonic generation," Opt. Lett. 33, 1099-1101 (2008).

[20] A. Ozawa, J. Rauschenberger, C. Gohle, M. Herrmann, D. R. Walker, V. Pervak, A. Fernandez, R. Graf, A. Apolonski, R. Holzwarth, F. Krausz, T. Hänsch, and T. Udem, "High Harmonic Frequency Combs for High Resolution Spectroscopy," Phys. Rev. Lett. 100, 253901 (2008).

[21] A. Cingöz, D. C. Yost, J. Ye, A. Ruehl, M. Fermann, and I. Hartl, "Power Scaling of High-Repetition-Rate HHG," International Conference on Ultrafast Phenomena (2010).

[22] B. Bernhardt, A. Ozawa, I. Pupeza, A. Vernaleken, Y. Kobayashi, R. Holzwarth, E. Fill, F. Krausz, T. W. Hänsch, and T. Udem, "Green Enhancement Cavity for Frequency Comb Generation in the Extreme Ultraviolet," CLEO, paper QTuF3 (2011).

[23] A. Ozawa and Y. Kobayashi, "Intracavity high harmonic generation driven by Ybfiber based MOPA system at $80 \mathrm{MHz}$ repetition rate," CLEO, paper CThB4 (2011).

[24] T. Brabec and F. Krausz, "Intense few-cycle laser fields: Frontiers of nonlinear optics," Rev. Mod. Phys. 72, 545 (2000).

[25] F. Krausz and M. Ivanov, "Attosecond physics," Rev. Mod. Phys. 81, 163 (2009).

[26] A. Barty, S. Boutet, M. Bogan, S. Hau-Riege, S. Marchesini, K. Sokolowski-Tinten, N. Stojanovic, R. Tobey, H. Ehrke, A. Cavalleri, S. Düsterer, M. Frank, S. Bajt, B. W. Woods, M. M. Seibert, J. Hajdu, R. Treusch, and H. N. Chapman, "Ultrafast single-shot diffraction imaging of nanoscale dynamics," Nature Phot. 2, 415 (2008). 
[27] P. W. Wachulak, M. C. Marconi, R. A. Bartels, C. S. Menoni, and J. J. Rocca, "Soft X-ray laser holography with wavelength resolution," Opt. Express 15, 10622 (2007).

[28] J. Filevich, K. Kanizay, M. C. Marconi, J. L. A. Chilla, and J. J. Rocca, "Dense Plasma Diagnostics with an Amplitude-Division Soft-X-Ray Laser Interferometer Based on Diffraction Gratings," Opt. Lett. 25, 356 (2000).

[29] P. C. Hinnen, S. E. Werners, S. Stolte, W. Hogervorst, and W. Ubachs, "XUV-laser spectroscopy of HD at 92-98 nm," Phys. Rev. A 52, 4425 (1995).

[30] J. Lin, N. Weber, J. Maul, S. Hendel, K. Rott, M. Merkel, G. Schoenhense, and U. Kleineberg, "At-wavelength inspection of sub-40 nm defects in extreme ultraviolet lithography mask blank by photoemission electron microscopy," Opt. Lett. 32, 1875 (2007).

[31] A. L. Cavalieri, N. Müller, T. Uphues, V. S. Yakovlev, A. Baltuska, B. Horvath, B. Schmidt, L. Blümel, R. Holzwarth, S. Hendel, M. Drescher, U. Kleineberg, P. M. Echenique, R. Kienberger, F. Krausz, and U. Heinzmann, "Attosecond spectroscopy in condensed matter," Nature 449, 1029 (2007).

[32] I. Hartl, T. R. Schibli, A. Marcinkevicius, D. C. Yost, D. D. Hudson, M. E. Fermann, and J. Ye, "Cavity-enhanced similariton Yb-fiber laser frequency comb: $3 \times 10^{14} \mathrm{~W} / \mathrm{cm}^{2}$ peak intensity at $136 \mathrm{MHz}$," Opt. Lett. 32, $2870(2007)$.

[33] I. Pupeza, T. Eidam, J. Rauschenberger, B. Bernhardt, A. Ozawa, E. Fill, A. Apolonski, T. Udem, J. Limpert, Z. A. Alahmed, A. M. Azzeer, A. Tünnermann, T. W. Hänsch, and F. Krausz, "Power scaling of a high repetition rate enhancement cavity," Opt. Lett. 12, 2052 (2010).

[34] I. Pupeza, X. Gu, E. Fill, T. Eidam, J. Limpert, A. Tünnermann, F. Krausz, and T. Udem, "Highly Sensitive Dispersion Measurement of a High-Power Passive Optical Resonator Using Spatial-Spectral Interferometry," Opt. Express 18, 26184 (2010).

[35] I. Pupeza, E. Fill, and F. Krausz, "Low-loss VIS/IR-XUV beam splitter for highpower applications," Opt. Express 19, 12108 (2011).

[36] Y.-Y. Yang, F. Süssmann, S. Zherebtsov, I. Pupeza, J. Kaster, D. Lehr, E.-B. Kley, E. Fill, X.-M. Duan, Z.-S. Zhao, F. Krausz, S. Stebbings, and M. F. Kling, "Optimization and characterization of a highly-efficient diffraction nanograting for $\mathrm{MHz}$ XUV pulses," Opt. Express 19, 1955 (2011).

[37] J. Weitenberg, P. Russbüldt, T. Eidam, and I. Pupeza, "Transverse mode tailoring in a quasi-imaging high-finesse femtosecond enhancement cavity," Opt. Express 19, 9551 (2011). 
[38] T. Udem, J. Reichert, R. Holzwarth, and T. W. Hänsch, "Absolute Optical Frequency Measurement of the Cesium $D_{1}$ Line with a Mode-Locked Laser," Phys. Rev. Lett. 82, 3568 (1998).

[39] T. W. Hänsch, R. Holzwarth, J. Reichert, and T. Udem, "Measuring the frequency of light with a femtosecond laser frequency comb," Proceedings of the International School of Physics Enrico Fermi, Course CXLVI p. 747 (2001).

[40] T. Udem, R. Holzwarth, and T. W. Hänsch, "Optical frequency metrology," Nature 16, 233 (2002).

[41] S. T. Cundiff, "Phase stabilization of ultrashort optical pulses," J. Phys. D: Appl. Phys. 35, R43 (2002).

[42] S. T. Cundiff, "Femtosecond Comb Technology," Journal of the Korean Phys. Soc. 48, 1181 (2006).

[43] T. R. Schibli, I. Hartl, D. C. Yost, M. J. Martin, A. Marcinkevicius, M. E. Fermann, and J. Ye, "Optical frequency comb with submillihertz linewidth and more than $10 \mathrm{~W}$ average power," Nature Phot. 2, 355 (2008).

[44] J.-C. Diels and W. Rudolph, Ultrashort Laser Pulse Phenomena (Academic Press, 1996).

[45] C. Rullière, Femtosecond Laser Pulses, Principles and Experiments (Springer, 2005).

[46] H. A. Haus, J. G. Fujimoto, and E. P. Ippen, "Analytic Theory of Additive Pulse and Kerr Lens Mode Locking," IEEE J. Quantum Electron. 28, 2086 (1992).

[47] E. P. Ippen, "Principles of passive mode locking," Applied Physics B: Lasers and Optics 58, 159 (1994).

[48] A. Baltuska, M. Uiberacker, E. Goulielmakis, R. Kienberger, V. Yakovlev, T. Udem, T. Hänsch, and F. Krausz, "Phase-controlled amplification of few-cycle laser pulses," Selected Topics in Quantum Electronics, IEEE Journal of 9, 972 (2003).

[49] L. Xu, C. Spielmann, A. Poppe, T. Brabec, F. Krausz, and T. W. Hänsch, "Route to phase control of ultrashort light pulses," Opt. Lett. 21, 2008 (1996).

[50] T. Brabec, Strong field laser physics (Springer, Cambridge, 2008).

[51] J. C. Petersen and A. N. Luiten, "Short pulses in optical resonators," Opt. Express 11, 2975 (2003).

[52] K. W. Holman, R. J. Jones, A. Marian, S. T. Cundiff, and J. Ye, "Intensity-related dynamics of femtosecond frequency combs," Opt. Lett. 28, 851 (2003). 
[53] D. R. Walker, T. Udem, C. Gohle, B. Stein, and T. W. Hänsch, "Frequency dependence of the fixed point in a fluctuating frequency comb," Appl. Phys. B 89, 535 (2007).

[54] R. L. Barger, M. S. Sorem, and J. Hall, "Frequency stabilization of a cw dye laser," Appl. Phys. Lett. 22, 573 (1973).

[55] T. Hänsch and B. Couillaud, "Laser Frequency Stabilization by Polarization Spectroscopy of a Reflecting Reference Cavity," Opt. Comm. 35, 441 (1980).

[56] C. E. Wieman and S. L. Gilbert, "Laser-frequency stabilization using mode interference from a reflecting reference interferometer," Opt. Lett. 7, 480 (1982).

[57] M. D. Harvey and A. G. White, "Frequency locking by analysis of orthogonal modes," Opt. Communications 221, 163 (2003).

[58] D. A. Shaddock, M. B. Gray, and D. E. McClelland, "Frequency locking a laser to an optical cavity by use of spatial mode interference," Opt. Lett. 24, 1499 (1999).

[59] R. W. P. Drever, J. L. Hall, F. V. Kowalski, J. Hough, G. M. Ford, A. J. Munley, and H. Ward, "Laser phase and frequency stabilization using an optical resonator," Appl. Phys. B: Photophys. Laser Chem. 31, 97 (1983).

[60] E. D. Black, "An introduction to Pound-Drever-Hall laser frequency stabilization," Am. J. Phys. 69, 79 (2001).

[61] R. J. Jones, I. Thomann, and J. Ye, "Precision stabilization of femtosecond lasers to high-finesse optical cavities," Phys. Rev. A 69, 051803 (2004).

[62] R. V. Pound, "Electronic Frequency Stabilization of Microwave Oscillators," Rev. Sci. Instrum. 17, 490 (1946).

[63] R. Ell, U. Morgener, F. X. Kärtner, J. G. Fujimoto, E. P. Ippen, V. Scheuer, G. Angelov, T. Tschudi, M. J. Lederer, A. Boiko, and B. Luther-Davies, "Generation of 5 -fs pulses and octave-spanning spectra directly from a Ti:sapphire laser," Opt. Lett. 26, 373 (2001).

[64] R. Ell, J. R. Birge, M. Araghchini, and F. X. Kärtner, "Carrier-envelope phase control by a composite plate," Opt. Express 14, 5829 (2006).

[65] N. Haverkamp, H. Hundertmark, C. Fallnich, and H. R. Telle, "Frequency stabilization of mode-locked Erbium fiber lasers using pump power control," Appl. Phys. B 78, 321 (2004).

[66] W.-Y. Cheng, T.-H. Wu, S.-W. Huang, S.-Y. Lin, and C.-M. Wu, "Stabilizing the frequency of femtosecond Ti:sapphire comb laser by a novel scheme," Applied Physics B: Lasers and Optics 92, 13 (2008). 
[67] I. Pupeza, T. Eidam, J. Kaster, B. Bernhardt, J. Rauschenberger, A. Ozawa, E. Fill, T. Udem, M. F. Kling, J. Limpert, Z. A. Alahmed, A. M. Azzeer, A. Tünnermann, T. W. Hänsch, and F. Krausz, "Power scaling of femtosecond enhancement cavities and high-power applications," Proc. SPIE 7914, 79 141I (2011).

[68] K. An, C. Yang, R. R. Dasari, and M. S. Feld, "Cavity ring-down technique and its application to the measurement of ultraslow velocities," Opt. Lett. 20, 1068 (1995).

[69] R. Paschotta, "Beam quality deterioration of lasers caused by intracavity beam distortions," Opt. Express 14, 6069 (2006).

[70] T. K. Allison, A. Cingöz, D. C. Yost, and J. Ye, "Cavity extreme nonlinear optics," in preparation, preprint: arXiv:1105.4195 (2011).

[71] C. Gohle, "A Coherent Frequency Comb in the Extreme Ultraviolet," Dissertation, MPQ/LMU (2006).

[72] J. Rauschenberger, "Phase-stabilized Ultrashort Laser Systems for Spectroscopy," Dissertation, MPQ/LMU (2007).

[73] A. Ozawa, "Frequency combs in the extreme ultraviolet," Dissertation, MPQ/LMU (2009).

[74] A. McPherson, G. Gibson, H. Jara, U. Johann, T. S. Luk, I. A. McIntyre, K. Boyer, and C. K. Rhodes, "Studies of multiphoton production of vacuum-ultraviolet radiation in the rare gases," J. Opt. Soc. Am B 4, 595 (1987).

[75] M. Ferray, A. L'Huillier, X. F. Li, L. A. Lomprk, G. Mainfray, and C. Manus, "Multiple-harmonic conversion of $1064 \mathrm{~nm}$ radiation in rare gases," J. Phys. B: At. Mol. Opt. Phys. 21, L31-L35 (1988).

[76] P. B. Corkum, "Plasma Perspective on Strong-Field Multiphoton Ionization," Phys. Rev. Lett. 71, 1994 (1993).

[77] M. Lewenstein, P. Balcou, M. Y. Ivanov, A. L'Huillier, and P. B. Corkum, "Theory of high-harmonic generation by low-frequency laser fields," Phys. Rev. A 49, 2117 (1994).

[78] M. F. Kling and M. J. J. Vrakking, "Attosecond Electron Dynamics," Annu. Rev. Phys. Chem. 59, 463 (2008).

[79] P. Salieres, A. L'Huillier, and M. Lewenstein, "Theory of high-harmonic generation by low-frequency laser fields," Phys. Rev. Lett. 74, 3776 (1995).

[80] E. Constant, D. Garzella, P. Breger, E. Mevel, C. Dorrer, C. L. Blanc, F. Salin, and P. Agostini, "Optimizing High Harmonic Generation in Absorbing Gases: Model and Experiment," Phys. Rev. Lett. 82, 1668 (1999). 
[81] J.-F. Hergott, M. Kovacev, H. Merdji, C. Hubert, Y. Mairesse, E. Jean, P. Breger, P. Agostini, B. Carre, and P. Salieres, "Extreme-ultraviolet high-order harmonic pulses in the microjoule range," Phys. Rev. A 66, 021 801(R) (2002).

[82] E. Takahashi, Y. Nabekawa, M. Nurhuda, and K. Midorikawa, "Generation of highenergy high-order harmonics by use of a long interaction medium," J. Opt. Soc. Am B 20, 158 (2003).

[83] P. Balcou and A. L'Huillier, "Phase-matching effects in strong-field harmonic generation," Phys. Rev. A 47, 1447 (1993).

[84] A. L'Huillier, X. F. Li, and L. A. Lompré, "Propagation eefects in high-order harmonic generation in rare gases," J. Opt. Soc. Am B 7, 527 (1990).

[85] E. L. Falcao-Filho, V. M. Gkortsas, A. Gordon, and F. X. Kärtner, "Analytic scaling analysis of high harmonic generation conversion efficiency," Opt. Express 17, 11217 (2009).

[86] J. G. Eden, "High-order harmonic generation and other intense optical field-matter interactions: review of recent experimental and theoretical advances," Progress in Quantum Electronics 28, 197 (2004).

[87] J. Tate, T. Auguste, H. G. Muller, P. Salieres, P. Agostini, and L. F. DiMauro, "Scaling of Wave-Packet Dynamics in an Intense Midinfrared Field," Phys. Rev. Lett. 98, 013901 (2007).

[88] A. D. Shiner, C. Trallero-Herrero, N. Kajumba, H.-C. Bandulet, D. Comtois, F. Legare, M. Giguere, J.-C. Kieffer, P. B. Corkum, and D. M. Villeneuve, "Wavelength Scaling of High Harmonic Generation Efficiency," Phys. Rev. Lett. 103, 073902 (2009).

[89] P. Balcou, C. Cornaggia, A. S. L. Gomes, L. A. Lompré, and A. L'Huillier, "Optimizing high-order harmonic generation in strong fields," J. Phys. B: At. Mol. Opt. Phys. 25, 4467 (1992).

[90] K. Kondo, N. Sarukura, K. Sajiki, and S. Watanabe, "High-order harmonic generation by ultrashort KrF and Ti:sapphire lasers," Phys. Rev. A 47, R2480 (1993).

[91] J. Zhou, J. Peatross, M. M. Murnane, and H. C. Kapteyn, "Enhanced High-Harmonic Generation Using 25 fs Laser Pulses," Phys. Rev. Lett. 76, 752 (1996).

[92] K. D. Moll, R. J. Jones, and J. Ye, "Nonlinear dynamics inside femtosecond enhancement cavities," Opt. Express 13, 1672 (2005).

[93] V. L. Kalashnikov, "Femtosecond pulse enhancement in an external resonator: impact of dispersive and nonlinear effects," Appl. Phys. B 92, 19 (2008). 
[94] D. Carlson, J. Lee, J. Mongelli, E. Wright, and R. Jones., "Intracavity ionization and pulse formation in femtosecond enhancement cavities," submitted to Opt. Lett. (2011).

[95] M. Geissler, G. Tempea, A. Scrinzi, M. Schnürer, F. Krausz, and T. Brabec, "Light Propagation in Field-Ionizing Media: Extreme Nonlinear Optics," Phys. Rev. Lett. 83, 2930 (1992).

[96] R. Holzwarth, personal communication.

[97] S. Holzberger, personal communication.

[98] T. Eidam, F. Röser, O. Schmidt, J. Limpert, and A. Tünnermann, " 57 W, 27 fs pulses from a fiber laser system using nonlinear compression," Appl. Phys. B 92, 9 (2008).

[99] T. C. Briles, D. C. Yost, A. Cingöz, J. Ye, and T. R. Schibli, "Simple piezoelectricactuated mirror with $180 \mathrm{kHz}$ servo bandwidth," Opt. Express 18, 9739 (2010).

[100] T. Clausnitzer, J. Limpert, K. Zöllner, H. Zellmer, H. J. Fuchs, E. B. Kley, A. Tünnermann, M. Jupe, and D. Ristau, "Highly efficient transmission gratings in fused silica for chirped-pulse amplification systems," Appl. Optics 42, 6934 (2003).

[101] G. Stobrawa, "Aufbau und Anwendungen eines hochaufösenden Impulsformers zur Kontrolle ultrakurzer Laserimpulse," Dissertation, Friedrich-Schiller-Universität Jena (2003).

[102] M. Sheik-Bahae, A. A. Said, and E. W. V. Styrland, "High-sensitivity, single-beam $n_{2}$ measurements," Opt. Lett. 14, 955 (1989).

[103] M. Sheik-Bahae, A. A. Said, T. H. Wei, D. J. Hagan, and E. W. V. Styrland, "Sensitive measurement of optical nonlinearities using a single beam," IEEE J. Quantum Electron. 26, 760 (1990).

[104] T. Y. F. Tsang, "Optical third-harmonic generation at interfaces," Phys. Rev. A 52, 4116 (1995).

[105] A. Ozawa, personal communication.

[106] K. Moll, R. Jones, and J. Ye, "Output coupling methods for cavity-based highharmonic generation," Opt. Express 14, 8189 (2006).

[107] D. Esser, W. Bröring, J. Weitenberg, and H.-D. Hoffmann, "Laser-manufactured mirrors for geometrical output coupling of intracavity-generated high harmonics," manuscript in preparation . 
[108] O. Pronin, V. Pervak, E. Fill, J. Rauschenberger, F. Krausz, and A. Apolonski, "Ultrabroadband efficient intracavity XUV output coupler," Opt. Express 19, 10232 (2011).

[109] B. Bernhardt, personal communication.

[110] A. Vernaleken, personal communication.

[111] C. Altucci, T. Starczewski, E. Mevel, C.-G. Wahlström, B. Carré, and A. L'Huillier, "Influence of atomic density in high-order harmonic generation," J. Opt. Soc. Am B 13, 148 (1996).

[112] E. D. Palik, G. Ghosh, and E. J. Prucha, Handbook of Optical Constants of Solids (Academic Press, New York, 1985).

[113] Center for X-ray Optics (http://www.cxro.lbl.gov).

[114] E. Fill, personal communication.

[115] J. Kaster, I. Pupeza, T. Eidam, C. Jocher, E. Fill, J. Limpert, R. Holzwarth, B. Bernhardt, T. Udem, T. W. Hänsch, A. Tünnermann, and F. Krausz, "Towards MW Average Powers in Ultrafast High-Repetition-Rate Enhancement Cavities," HILAS, paper HFB4 (2011).

[116] S. V. Fomichev, P. Breger, B. Carre, P. Agostini, and D. F. Zaretski, "Non-collinear high harmonic generation," Laser Phys. 12, 383 (2002).

[117] A. Ozawa, A. Vernaleken, W. Schneider, I. Gotlibovych, T. Udem, and T. W. Hänsch, "Non-collinear high harmonic generation: a promising outcoupling method for cavityassisted XUV generation," Opt. Express 16, 6233 (2008).

[118] R. Hauck, H. P. Kortz, and H. Weber, "Misalignment sensitivity of optical resonators," Appl. Optics 19, 598 (1980).

[119] V. Magni, "Resonators for solid-state lasers with large-volume fundamental mode and high alignment stability," Appl. Optics 25, 107 (1986).

[120] T. Eidam, S. Hanf, E. Seise, T. V. Andersen, T. Gabler, C. Wirth, T. Schreiber, J. Limpert, and A. Tünnermann, "Femtosecond fiber CPA system emitting 830W average output power," Opt. Lett. 35, 94 (2010).

[121] P. Russbüldt, T. Mans, G. Rotarius, J. Weitenberg, H. D. Hoffmann, and R. Poprawe, "400W Yb:YAG Innoslab fs-Amplifier," Opt. Express 17, 12230 (2009).

[122] P. Russbüldt, T. Mans, J. Weitenberg, H. D. Hoffmann, and R. Poprawe, "Compact diode-pumped $1.1 \mathrm{~kW}$ Yb:YAG Innoslab femtosecond amplifier," Opt. Lett. 35, 4169 (2010). 
[123] A. Ozawa, T. Udem, U. D. Zeitner, T. W. Hänsch, and P. Hommelhoff, "Modeling and optimization of single-pass laser amplifiers for high-repetition-rate laser pulses," Phys. Rev. A 82, 033815 (2010).

[124] A. Vernaleken, J. Weitenberg, T. Sartorius, P. Russbüldt, W. Schneider, S. L. Stebbings, M. F. Kling, P. Hommelhoff, H.-D. Hoffmann, R. Poprawe, F. Krausz, T. W. Hänsch, and T. Udem, "Single pass high harmonic generation at $20.8 \mathrm{MHz}$ repetition rate," Opt. Lett. 36, 3428 (2011).

[125] J. Weitenberg, personal communication.

[126] E. Goulielmakis, M. Schultze, M. Hofstetter, V. S. Yakovlev, J. G. M. Uiberacker, A. L. Aquila, E. M. Gullikson, D. T. Attwood, R. Kienberger, F. Krausz, and U. Kleineberg, "Single-Cycle Nonlinear Optics," Science 320, 1614 (2008).

[127] I. J. Sola, E. Mevel, L. Elouga, E. Constant, V. Strelkov, L. Poletto, P. Villoresi, E. Benedetti, J.-P. Caumes, S. Stagira, C. Vozzi, G. Sansone, and M. Nisoli, "Controlling attosecond electron dynamics by phase-stabilized polarization gating," Nature Phys. 2, 319 (2006).

[128] M. Fiess, B. Horvath, T. Wittmann, W. Helml, Y. Cheng, B. Zeng, Z. Xu, A. Scrinzi, J. Gagnon, F. Krausz, and R. Kienberger, "Attosecond control of tunneling ionization and electron trajectories," New J. of Physics 13, 033031 (2011).

[129] M. J. Abel, T. Pfeifer, P. M. Nagel, W. Boutu, M. J. Bell, C. P. Steiner, D. M. Neumark, and S. R. Leone, "Isolated attosecond pulses from ionization gating of high-harmonic emission," Chem. Phys. 366, 9 (2009).

[130] H. Mashiko, S. Gilbertson, C. Li, S. D. Khan, M. M. Shakya, E. Moon, and Z. Chang, "Double Optical Gating of High-Order Harmonic Generation with Carrier-Envelope Phase Stabilized Lasers," Phys. Rev. Lett. 100, 103906 (2008).

[131] X. Feng, S. Gilbertson, H. Mashiko, H. Wang, S. D. Khan, M. Chini, Y. Wu, K. Zhao, and Z. Chang, "Generation of Isolated Attosecond Pulses with 20 to 28 Femtosecond Lasers," Phys. Rev. Lett. 103, 183901 (2009).

[132] S. Gilbertson, Y. Wu, S. D. Khan, M. Chini, K. Zhao, X. Feng, and Z. Chang, "Isolated attosecond pulse generation using multicycle pulses directly from a laser amplifier," Phys. Rev. A 81, 043810 (2010).

[133] M. Durach, A. Rusina, M. F. Kling, and M. I. Stockman, "Metallization of Nanofilms in Strong Adiabatic Electric Fields," Phys. Rev. Lett. 105, 086803 (2010).

[134] M. Durach, A. Rusina, M. F. Kling, and M. I. Stockman, "Ultrafast Dynamic Matallization of Dielectric Nanofilms by Strong Single-Cycle Optical Fields," submitted for publication (2011). 
[135] F. V. Hartemann, W. J. Brown, D. J. Gibson, S. G. Anderson, A. M. Tremaine, P. T. Springer, A. J. Wootton, E. P. Hartouni, and C. P. J. Barty, "High-energy scaling of Compton scattering light sources," Phys. Rev. ST Accel. Beams 8, 100702 (2005).

[136] K. Sakaue, M. Washio, S. Araki, M. Fukuda, Y. Higashi, Y. Honda, T. Omori, T. Taniguchi, N. Terunum, J. Urakawa, and N. Sasao, "Observation of pulsed X-ray trains produced by laser-electron Compton scatterings," Rev. Scientific. Instrum. 80, 123304 (2009).

[137] R. Krischek, W. Wieczorek, A. Ozawa, N. Kiesel, P. Michelberger, T. Udem, and H. Weinfurter, "Ultraviolet enhancement cavity for ultrafast nonlinear optics and high-rate multiphoton entanglement experiments," Nature Phot. 4, 170 (2010).

[138] M. Motsch, M. Zeppenfeld, P. W. H. Pinkse, and G. Rempe, "Cavity-Enhanced Rayleigh Scattering," New J. of Physics 12, 063022 (2010).

[139] C. Gohle, B. Stein, A. Schliesser, T. Udem, and T. W. Hänsch, "Frequency Comb Vernier Spectroscopy for Broadband, High Resolution, High Sensitivity Absorption and Dispersion Spectra," Phys. Rev. Lett. 99, 263902 (2007).

[140] M. Herrmann, M. Haas, U. D. Jentschura, F. Kottmann, D. Leibfried, G. Saathoff, C. Gohle, A. Ozawa, V. Batteiger, S. Knünz, N. Kolachevsky, H. A. Schüssler, T. W. Hänsch, and T. Udem, "Feasibility of coherent xuv spectroscopy on the $1 S-2 S$ transition in singly ionized helium," Phys. Rev. A 79, 052505 (2009).

[141] A. Zavatta, V. Parigi, and M. Bellini, "Towards quantum frequency combs: Boosting the generation of highly nonclassical light states by cavity-enhanced parametric downconversion at high repetition rates," Phys. Rev. A 78, 033809 (2008).

[142] G. Rempe, R. J. Thompson, H. J. Kimble, and R. Lalezari, "Measurement of ultralow losses in an optical interferometer," Opt. Lett. 17, 363 (1992).

[143] J. Weitenberg, P. Russbüldt, I. Pupeza, T. Udem, H.-D. Hoffmann, and R. Poprawe, "Geometrical on-axis access to high-finesse resonators by quasi-imaging," manuscript in preparation .

The publications [33 37] are included in this cumulative thesis. They cite references not listed here. 


\section{Acknowledgements}

Zuallererst möchte ich Prof. Ferenc Krausz für seine ständige, unermüdliche Unterstützung herzlich danken, die weit über das Fachliche hinausreichte und den Lernprozess und meine Kreativität in den letzten vier Jahren enorm förderte.

Jens Rauschenberger führte mich als Diplommathematiker und Diplomingenieur in die Laserlabore der Experimentalphysik ein - danke für Deine Geduld! Des Weiteren sind die Projekte, die den finanziellen Rahmen der Forschung meiner Doktorarbeit sicherten, maßgeblich seiner Tatkraft zu verdanken.

Ernst Fill möchte ich besonders für seine ständige Inspiration, fundiert von solidem und weitreichendem Fachwissen danken und für seine hochmotivierende Art. Die Zusammenarbeit mit Ihnen war für mich eine große Freude! Vielen Dank auch für das wiederholte Korrekturlesen dieser Arbeit und die schönen Verbesserungsvorschläge!

Herzlich danken möchte ich auch meinen Kollegen Jan Kaster und Simon Holzberger, ohne die es zu dieser Arbeit, in dieser Form, nicht hätte kommen können. Sie haben nicht nur mit voller Begeisterung an meiner Seite geforscht, sondern mir auch unzählige Dinge beigebracht, für die ich sehr dankbar bin.

Ein besonderes "Danke schön!" gilt der Faserlaser Gruppe vom IAP Jena, die das Lasersystem Jenny zur Verfügung stellte, das diese Arbeit ermöglichte: Prof. Andreas Tünnermann, Prof. Jens Limpert, Tino Eidam und Fabian Röser. Tino möchte ich besonders für die vielen Besuche in München danken, im Rahmen derer er immer wieder die Jenny reparierte und upgradete. Wir haben zusammen viel experimentiert und gelernt. Die vielen interessanten Gespräche haben einen wichtigen Teil zu meiner Motivation in diesen Jahren beigetragen, Action Jackson!

Von den "Hänschies" erhielt ich großartige Unterstützung. Besonders möchte ich Prof. Hänsch, Thomas Udem, Ronald Holzwarth, Birgittta Bernhardt, Akira Ozawa und Andreas Vernaleken danken. Und ganz besonders möchte ich Gitti für ihre einzigartige Unterstützung danken. Sie stand immer mit Rat, Tat und Komponenten an meiner Seite. Thomas möchte ich auch ganz besonders danken: er fand immer Zeit und Begeisterung für meine Fragen und Ideen und gab meistens sehr gute Antworten und Anregungen! Euch beiden möchte ich herzlich danken, dass Ihr mir so vieles beigebracht habt! Ich möchte noch Christoph Gohle, einem der beeindruckendsten Wissenschaftler, die ich kenne, herzlich für die Zeit danken, die er sich für Gespräche über Physik vor meiner Prüfung nahm.

One of the greatest collaborations during these years was a brief, but very pleasant and fruitful one with Xun Gu. Xun, thanks a lot for the inspiring way of tackling the problems and solving them!

Johannes Weitenberg und Peter Rußbüldt vom Fraunhofer ILT möchte ich für die vielen interessanten Diskussionen danken. Vor allem Johannes inspiriert mich immer wieder mit seinem Zugang und seiner Leidenschaft für die Wissenschaft. Die Wochen unserer gemeinsamen Experimente gehören zu den schönsten meiner Promotionszeit und ich hoffe, es werden sich noch Gelegenheiten zum gemeinsamen Experimentieren ergeben! Dominik Esser möchte auch herzlich für die ergebnisreichen Anstrengungen mit dem Bohren kleiner Löchern in Spiegel danken. 
Den Kollegen aus unserer großen "Krausz-Gruppe", möchte ich für ihr Interesse und Unterstützung danken, besonders Oleg Pronin, Vladimir Pervak, Alexander Apolonski und Matthias Kling. Für ihre tatkräftige Unterstützung möchte ich auch Herrn Siegfried Herbst und Herrn Rolf Öhm von der LMU danken.

I would like to send a special thanks to Prof. Abdallah M. Azzeer and Prof. Zeyad A. Alahmed from King Saud University in Riyadh. May our future collaborations be fruitful!

Während dieser Promotionszeit habe ich am MPQ und an der LMU viele Bekanntschaften und Freundschaften auch außerhalb des Labors geschlossen, beim Sport, Musizieren, Philosophieren oder einfach nur so. Für die schöne Zeit möchte ich insbesondere: Roswitha Graf, Catherine Teisset, Thomas Ganz, Friedrich Kirchner, Raphael Weingartner, Justin Gagnon (einen besonderen Dank für die interessanten Gespräche über Quantenmechanik!), Alex Buck, Peter Jazz, Sergey Rykovanov, John Frusciante, Sarah Stebbings, Fabian Lücking, Peter Reckenthäler, Michael Hofstetter, Casey Chow, Janis Alnis danken und mich bei allen entschuldigen, die ich vergessen habe, hier zu erwähnen.

Außerhalb der MPQ/LMU Welt gebührt ein ganz besonderer Dank meinen Professoren und Mentoren, die mich ermutigten, den wissenschaftlichen Weg der letzten vier Jahre einzuschlagen und mich dafür vorbereiteten. Ich möchte Prof. Rainer Löwen für seine einzigartige Unterstützung während des Mathematikstudiums danken und dafür, dass er mich seine Art, Wissenschaft zu leben, erfahren ließ, besonders im Zusammenhang mit meiner Diplomarbeit im Bereich der Inzidenzgeometrie. Prof. Martin Koch möchte ich für die schönen Jahre der Beschäftigung mit THz in Braunschweig danken und dafür, dass er mir riet, mich für diese Promotionsstelle zu bewerben. Prof. Alek Kavcic möchte ich für die Gelegenheit danken, meine Diplomarbeit in der Codierungstheorie an der Harvard University zu schreiben und für die damit verbundene Betreuung, sowie für die umfangreiche Einführung in die Kunst, wissenschaftliche Texte zu verfassen. Prof. Werner Deutsch, meinem Vertrauensdozenten, möchte ich für die vielen weisen Ratschläge und für seine Freundschaft danken.

Vielleicht der grösste Dank gebührt meiner Familie, die mir ununterbrochenen Rückhalt, Geborgenheit, Liebe, Mut und Heiterkeit schenkte. Besonders möchte ich Susi für die Liebe, Unterstützung und Geduld in den letzten Jahren danken, meiner Schwester Vivi für die Inspiration, Kraft und Lebensfreude, meinen Eltern: Vom Vater hab ich die Statur,/ Des Lebens ernstes Führen,/Vom Mütterchen die Frohnatur/ Und Lust zu fabulieren, wie Goethe so schön sagte. Zusätzlich möchte ich meinem Vater für die ständige Motivation zu dieser Arbeit danken und für die vielen Gespräche, die oft zu einzigartigen Ideen führen. Des Weiteren danke ich den Familien Rădulescu und Lera i Roura, die sich darum bemühten, mir die günstigsten Bedingungen für diese Promotion zu schaffen. Multumesc din inimă! Moltes gracies! 


\title{
Curriculum Vitae
}

\author{
Ioachim Pupeza
}

$\begin{array}{ll}\text { Geburtsdatum } & \text { 9. März 1980 } \\ \text { Geburtsort } & \text { Bukarest, Rumänien } \\ \text { Staatsangehörigkeit } & \text { deutsch }\end{array}$

\section{Werdegang}

1986 - $1997 \quad$ Besuch der Deutschen Schule Bukarest

1997 - $1999 \quad$ Besuch des Scharnhorstgymnasiums Hildesheim

1999 - $2006 \quad$ Studium zum Diplomingenieur an der

Technischen Universität in Braunschweig

2000 - $2007 \quad$ Studium zum Diplommathematiker an der

Technischen Universität in Braunschweig

2004 - $2005 \quad$ Visiting Fellow und Diplomarbeit in der Elektrotechnik

an der Harvard University, Cambridge, MA, USA

mit einem Stipendium der Studienstiftung des deutschen Volkes

2005 zweimonatiges Praktikum am Deutschen Zentrum für

Luft- und Raumfahrt in Oberpfaffenhofen

2007 - $2011 \quad$ Doktorand in der Gruppe von Prof. Ferenc Krausz am Max-Planck-Institut für Quantenoptik in Garching 


\section{Publications 2007-2011}

\section{Journal Publications}

- I. Pupeza, R. Wilk, M. Koch, "Highly accurate optical material parameter determination with THz time domain spectroscopy," Opt. Express 15, 4335-4350 (2007).

- R. Wilk, I. Pupeza, R. Cernat, M. Koch, "Highly Accurate THz Time-Domain Spectroscopy of Multi-Layer Structures," IEEE Journal of Selected Topics in Quantum Electronics 14, 391-398 (2008).

- T. Kleine-Ostmann, T. Schrader, M. Bieler, U. Siegner, C. Monte, B. Gutschwager, J. Hollandt, A. Steiger, L. Werner, R. Müller, G. Ulm, I. Pupeza, M. Koch, "THz Metrology," Frequenz 62, 137-148 (2008).

- M. A. Salhi, I. Pupeza, M. Koch, "Confocal THz Microscope," Journal of Infrared, Millimeter and Therahertz Waves 31, 358-366 (2009).

- C. Jördens, K. L. Chee, I. A. I. Al-Naib, I. Pupeza, S. Peik, G. Wenke, M. Koch, "Dielectric Fibres for Low-Loss Transmission of Millimetre Waves and its Application in Couplers and Splitters", Journal of Infrared, Millimeter and Therahertz Waves 31, 214-220 (2009).

- N. Krumbholz, T. Hochrein, N. Vieweg, I. Radovanovic, I. Pupeza, M. Schubert, K. Kretschmer, M. Koch, "Degree of Dispersion of Polymeric Compounds Determined with Terahertz Time-Domain Spectroscopy," Polymer Engineering and Science 51, 109-116 (2010).

- S. Immervoll, R. Löwen, I. Pupeza, "A local characterization of smooth projective planes," Proceedings of the American Mathematical Society 138, 323-332 (2010).

- I. Pupeza, T. Eidam, J. Rauschenberger, B. Bernhardt, A. Ozawa, E. Fill, A. Apolonski, Th. Udem, J. Limpert, Z. A. Alahmed, A. M. Azzeer, A. Tünnermann, T. W. Hänsch and F. Krausz, "Power scaling of a high repetition rate enhancement cavity," Opt. Letters 12, 2052-2054 (2010).

- I. Pupeza, X. Gu, E. Fill, T. Eidam, J. Limpert, A. Tünnermann, F. Krausz, Th. Udem, "Highly sensitive dispersion measurement of a high-power passive optical resonator using spatial-spectral interferometry," Opt. Express 18, 2618-26295 (2010).

- I. Pupeza, T. Eidam, J. Kaster, B. Bernhardt, J. Rauschenberger, A. Ozawa, E. Fill, Th. Udem, M. F. Kling, J. Limpert, Z. A. Alahmed, A. M. Azzeer, A. Tünnermann, Th. W. Hänsch, F. Krausz, "Power scaling of femtosecond enhancement cavities and high-power applications," Proc. SPIE 7914, Paper 79141I (2011). 
- Y.-Y. Yang, F. Süßmann, S. Zherebtsov, I. Pupeza, J. Kaster, D. Lehr, H.-J. Fuchs, E.-B. Kley, E. Fill, X.-M. Duan, Z.-S. Zhao, F. Krausz, S. L. Stebbings, M. F. Kling, "Optimization and characterization of a highly-efficient diffraction nanograting for MHz XUV pulses," Opt. Express 19, 1955-1962 (2011).

- J. Weitenberg, P. Rußbüldt, T. Eidam, I. Pupeza, "Transverse mode tailoring in a quasi-imaging high-finesse femtosecond enhancement cavity," Opt. Express 19, 95519561 (2011).

- I. Pupeza, E. Fill, F. Krausz, "Low-loss VIS/IR-XUV beam splitter for high-power applications," Opt. Express 19, 12108-12118 (2011).

- J. Weitenberg, P. Rußbüldt, I. Pupeza, Th. Udem, H.-D. Hoffmann, and R. Poprawe, "Geometrical on-axis access to high-finesse resonators by quasi-imaging," manuscript in preparation.

- B. Bernhardt, A. Ozawa, A. Vernaleken, I. Pupeza, J. Kaster, Y. Kobayashi, R. Holzwarth, E. Fill, F. Krausz, T. W. Hänsch, and Th. Udem, "First green enhancement cavity generating an ultra violet frequency comb," manuscript in preparation.

\section{Contributed and Invited Conference Talks (Selection)}

- I. Pupeza, R. Wilk, F. Rutz, M. Koch, "Highly Accurate Material Parameter Extraction from THz Time Domain Spectroscopy Data," CLEO (2007).

- I. Pupeza, J. Rauschenberger, T. Eidam, F. Röser, B. Bernhardt, A. Ozawa, R. Holzwarth, Th. Udem, J. Limpert, A. Apolonski, T.W. Hänsch, A. Tünnermann, F. Krausz, "Towards High-Power XUV Generation Using an Yb-Based Enhancement Cavity," CLEO/Europe (2009).

- I. Pupeza, T. Eidam, B. Bernhardt, A. Ozawa, O. Pronin, J. Rauschenberger, F. Röser, A. Apolonski, Th. Udem, J. Limpert, A. Tünnermann, T. W. Hänsch, F. Krausz, "High-Power Femtosecond Enhancement Cavities for XUV Generation," invited talk, International Laser Physics Workshop (2009).

- I. Pupeza, T. Eidam, O. Pronin, J. Rauschenberger, B. Bernhardt, A. Ozawa, Th. Udem, R. Holzwarth, J. Limpert, A. Apolonski, T. W. Hänsch, A. Tünnermann, F. Krausz, "Femtosecond High Repetition Rate External Cavity beyond the Average Power Limit for Linear Enhancement," ASSP (2010).

- I. Pupeza, T. Eidam, B. Bernhardt, A. Ozawa, J. Rauschenberger, E. Fill, A. Apolonski, Th. Udem, J. Limpert, Z. A. Alahmed, Abdallah M Azzeer, T. W. Hänsch, A. Tünnermann, F. Krausz, "Power Scaling of a 78 MHz-Repetition Rate Femtosecond Enhancement Cavity," CLEO (2010). 
- I. Pupeza, T. Eidam, J. Kaster, B. Bernhardt, J. Rauschenberger, A. Ozawa, E. Fill, Th. Udem, M. F. Kling, J. Limpert, Z. A. Alahmed, A. M. Azzeer, A. Tünnermann, T. W. Hänsch and F. Krausz, "Power Scaling and High-Power Applications of a Femtosecond Enhancement Cavity," invited talk, Photonics West (2011).

- I. Pupeza, J. Kaster, S. Holzberger, T. Eidam, B. Bernhardt, E. Fill, V. Pervak, A. Apolonski, Th. Udem, R. Holzwarth, J. Limpert, Z. A. Alahmed, A. M. Azzeer, A. Tünnermann, T. W. Hänsch and F. Krausz, "Generation of Brilliant XUV Radiation with Enhancement Cavities," invited talk, SIECPC (2011).

- I. Pupeza, J. Kaster, T. Eidam, B. Bernhardt, R. Holzwarth, T. W. Hänsch, Th. Udem, J. Limpert, A. Tünnermann, E. Fill, F. Krausz, "Progress in Enhancement Cavities for XUV Generation," CLEO (2011).

- I. Pupeza, J. Weitenberg, P. Rußbüldt, T. Eidam, J. Limpert, E. Fill, Th. Udem, H.D. Hoffmann, R. Poprawe, A. Tünnermann, F. Krausz, "Tailored Transverse Modes in High-Finesse Femtosecond Enhancement Cavities," CLEO (2011).

\section{Patent Applications}

- F. Krausz, E. Fill, J. Rauschenberger, I. Pupeza, "Method and laser device for generating pulsed high power laser light," Patent Application number PCT/EP2009/008278, (2009).

- J. Kaster, I. Pupeza, E. Fill, F. Krausz, "Method of generating enhanced intraresonator laser light, enhancement resonator and laser device," Patent Application number PCT/EP2010/005464, (2010).

- J. Weitenberg, P. Rußbüldt, Th. Udem, I. Pupeza, "Optischer Resonator mit direktem geometrischem Zugang auf der optischen Achse," Patent Application number 102011008225.5, (2011).

- I. Pupeza, F. Krausz, "Spatially splitting or combining radiation beams," Patent Application number 11000626.9, (2011). 